



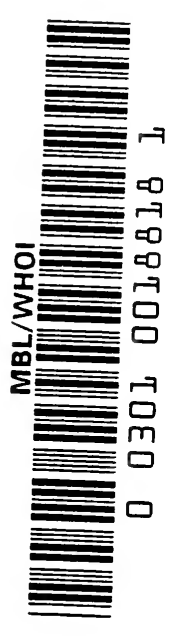





\title{
THE UNIVERSITY OF CHICAGO SCIENCE SERIES
}

\author{
Editorial Committee \\ ELIAKIM HASTINGS MOORE, Chairman \\ JOHN MERLE COULTER \\ ROBERT ANDREWS MILLIKAN
}




\section{He UNIVERSITY OF CHICAgo SCIENCE SERIES, established by the Trustees of the University, owes its origin to}

a belief that there should be a medium of publication occupying a position between the technical journals with their short articles and the elaborate treatises which attempt to cover several or all aspects of a wide field. The volumes of the series will differ from the discussions generally appearing in technical journals in that they will present the complete results of an experiment or series of investigations which previously have appeared only in scattered articles, if published at all. On the other hand, they will differ from detailed treatises by confining themselves to specific problems of current interest, and in presenting the subject in as summary a manner and with as little technical detail as is consistent with sound method. They will be written not only for the specialist but for the educated layman. 
THE ORIGIN AND DEVELOPMENT OF THE NERVOUS SYSTEM

FROM A PHYSIOLOGICAL VIEWPOINT 
THE UNIVERSITY OF CHICAGO PRESS

CHICAGO, ILLINOIS

THE BAKER \& TAYLOR COMPANY

NEW YORK

THE CAMBRIDGE UNIVERSITY PRESS

LONDON

THE MARUZEN-KABUSHIKI-KAISHA

TOKYO, OSAKA, KYOTO, FUKUOKA, SENDAI

THE MISSION BOOK COMPANY

SHANGHAI 


\title{
The Origin and Development of the Nervous System
} FROM A PHYSIOLOGICAL VIEWPOINT

\author{
By \\ Charles Manning Child \\ Professor of Zö̈logy, University of Chicago
}

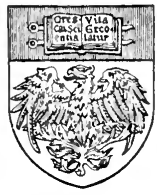

THE UNIVERSITY OF CHICAGO PRESS CHICAGO, ILLINOIS 
Copyright I92I By

The University of Chicago

All Rights Reserved

Published January, I92I 


\section{PREFACE}

Concerning the embryonic development and the evolution of nervous structure a large body of fact and hypothesis is at hand, but the problem of the origin of the nervous system as an organ of excitation, transmission, and integration has received much less attention. Irritability, that is, excitability, is commonly regarded as a fundamental property of living protoplasm, and it is often asserted that the nervous system does not represent a new function, added at a certain stage of development, or of evolution, to the original functional complex of the organism, but rather a development of the primitive mechanisms of excitation and transmission. Not infrequently, however, particularly within recent years, it has been maintained that the primary factors in physiological correlation and integration consist of chemical substances and their transportation by one means or another. These two conceptions are difficult to reconcile, for the one implies that excitation and transmission are primary factors in physiological correlation, the other that they are of secondary origin. As a matter of fact, we know that excitation and transmission occur in protoplasm in the absence of anything which we can identify as nervous structure; moreover, it is difficult to conceive a living organism incapable of at least some degree of excitation and transmission.

Considered from a physiological viewpoint, the origin of the nervous system must be sought in conditions present before the appearance of definitely recognizable 
morphological structure of nervous character. This book is first of all a consideration in the light of recent experimental investigation of certain of the physiological conditions which antedate the appearance of the nervous system, and with which its appearance and development appear to be closely associated. It is, in fact, an attempt to establish the existence of physiological continuity between the simple quantitative gradient in physiological condition and the nervous system. As I shall endeavor to show on the basis of experimental data, the physiological gradient arises in the final analysis as an excitation-transmission relation in response to the differential action of an external factor and at the same time represents the primary integrating factor of the sort characteristic of the organism. The nervous system represents the organ of integration par excellence, and some sort of continuity must exist between the primary factors concerned in determining those relations between regions or parts which make the organism an orderly whole and the nervous system, which continues the development of this integration to its highest point. Since the book is concerned first with this question of continuity and its nature and since no general consideration of the physiological gradients has appeared since I9I 5 , it has been found necessary to devote the earlier chapters to a discussion of pattern in the organism and its relation to the gradients. These chapters constitute the basis for the consideration of the nervous system in the stricter sense, which follows. Believing as I do that the conception of the physiological gradient enables us to throw some light, not only on the problem of development in general, but particularly on 
the problem of the nervous system in its physiological aspects, it has seemed a task worth undertaking, even though our knowledge is still fragmentary in many respects, to attempt to point out the relations between the gradients and the nervous system. As regards many points, of course, only hypotheses or suggestions are possible, but if these shall stimulate further investigation and so aid, even indirectly, in advancing our knowledge, their chief purpose will have been attained, whether they are confirmed or shown to be incorrect.

Since many different lines of investigation concerned in the discussion are of necessity mentioned only very briefly, it has seemed advisable to give references, so far as it could be done without unduly breaking the continuity of the text. The list of titles following the text makes no pretensions to completeness, but includes merely the publications to which reference is made.

I am deeply indebted to certain of my colleagues and co-workers in various ways: first of all to Dr. C. J. Herrick, who has played no small part in the development of the book. While it is by no means my intention to shift to his shoulders any of the responsibility for conclusions drawn or suggestions advanced, I feel it a great pleasure, as well as a duty to acknowledge my obligations to him for many fruitful suggestions made in the course of personal discussions during the years of our association in Chicago, for information on many questions of fact, for his kindness in reading and criticizing the entire manuscript, and finally for permission to reproduce certain figures. I am also under great obligation to Dr. L. H. Hyman of the Department of Zoölogy for reading and criticism of the manuscript 
and for permission to use unpublished data, and to Dr. A. W. Bellamy of the Department of Zoölogy for permission to use unpublished data and to reproduce certain figures.

For permission to reproduce published figures, acknowledgments are due further to the following publishers and authors: D. Appleton and Co.; Henry Holt and Co.; P. Blakiston's Son and Co.; The University of Chicago Press; Professor S. Ramon Cajal; Dr. F. R. Lillie; Dr. R. G. Harrison; Dr. W. A. Locy; Dr. J. B. Johnston; Dr. G. H. Parker; and Dr. Charles Hill. The sources of all figures reproduced are given in the legends. To the artist of the Department of Zoölogy, Mr. Kenji Toda, my thanks are due for his beautiful redrawing of figures reproduced.

C. M. Child

Chicago, Ill. June, I920 


\section{CONTENTS}

LIST OF ILLUSTRATIONS

PAGE

CHAPTER

I. The Problem of Pattern . . . . . . . . I

Pattern and Material. Organismic Pattern. The Origin of Organismic Pattern. Physiological Correlation as the Mechanism of Integration. Organismic Pattern and the Chromosomes. Conclusion.

II. The Physiological Basis of Axiate Pattern . The Physiological Gradient. The Evidence from Structure and Development. The Evidence from Susceptibility. The Evidence from Rate of Penetration. The Evidence from Certain Oxidation-Reduction Reactions. The Evidence from Bioelectric Phenomena. The Evidence from Oxygen Consumption and Carbon Dioxide Production. Evidence for the Existence of Physiological Gradients in Various Organs.

III. The Origin of Axiate Pattern in Nature and Experiment . . . . . . . . . . 50

The Origin of Polarity. The Origin of Symmetry. The Problem of Cell Pattern. The Relations of SurfaceInterior to Axiate Pattern. Conclusion.

IV. Excitation and Transiission in Relation to Axiate Pattern . . . . . . . . . .

The Excitation Gradient. Theories of Excitation and Transmission. Primitive Excitation Processes. The Question of Reversibility. General Discussion.

V. The Physiological Gradients in Relation to Organismic Integration · • • • • • .

The Physiological Gradient as a Basis for Differentiation. Physiological Deminance and Subordination in Relation to the Axial Gradients. Physiological Isolation. Conclusion. 
VI. Localization and Differentiation of Nervous Structure in Relation to the Organismic PATTERN

The Nervous System as a Product of Physiological Factors. The Localization of Nervous Structure. The Early Differentiation of Nervous Structure.

VII. The Physiological Relation of the Central Nervous System to Other Parts in DevelopMENT .

Physiological Independence and Dependence in Development in Relation to the Gradients. The Physiological Independence of the Apical Region and Head in Experimental Reproduction. The Relative Independence of the Nervous System in Development. The Signiiicance of the Facts.

VIII. The Nervous System in Relation to SegmenTATION

iX. Nervous Centralization and Cephalization in Evolution

Centralization in Relation to Axiation. Progressive Centralization and Cephalization in Various Groups. The "Stepladder" Type of Nervous System in the Invertebrates. The Position of Peripheral Receptors. Conclusion.

X. The Problear of Neuron Pattern . . .

The Neuron. The General Morphological Characteristics of Neuron Pattern. Neuron Pattern as a Case of Axiate Pattern. The Possible Factors Concerned in the Axiation of the Neuron. Kappers' Theory of the Origin of Neuron Pattern.

XI. Suggestions Concerning the Origin and Development of Neuron Pattern . . . . .

Electrical Polarization as a Localizing and Orienting Factor. Axon Development. Dendrite Development. The Neurons of the Spinal Ganglia. Kappers' "Neurobiotaxis" of the Cell Body. Certain "Monopolar" Neurons. The Cells of the Nervous Reticulum. Conclusion. 
XiI. Certain Features of Transmission and ConDuction in Relation to the Physiological Gradients . . . . . . . . . 206 The Development of Excitation and Conduction. Transmission in Certain Non-nervous or Neuroid Paths. Transmission in the Row of Swimming Plates of the Ctenophore. Certain Axiate Organs as Physiological Gradients. The "All-or-None" Principle in Nervous Conduction. Conduction in the Neuron and the Problem of the Synapse.

XIII. The Reflex Arc in Relation to the Gradient

The Physiological Characteristics of Reflex Arc and Excitation Gradient. Historical and Critical. The Functional Direction in the Nerve Net. Functional Direction in Certain Bilateral Invertebrates. Functional Direction in the Higher Animals. The Problem of the Origin and Development of the Cerebral Cortex.

Xiv. General Conclusions . . . . . . . 268

REFERENCES . . . . . . . . . . . . 273

INDEX . . • • • • • • • • • • • . 289 


\section{LIST OF ILLUSTRATIONS}

FIgs. I-3. Ovarian Eggs of Jellyfish, Phialidium gre-

garium, Indicating Relation between Polarity and Position . . . . . . . . 55

FIg. 4. Growing Oöcyte of Sternaspis scutata . . . $\quad 5^{6}$

FIg. 5. Semi-diagrammatic View of Ovarian Egg of Frog, Indicating Relation between Polarity and Circulation . . . . . . . . 56

FIG. 6. Diagram Indicating the Electric Currents Arising in Relation to an Excitation Gradient

Fig. 7. Diagram Illustrating Transmission up a Gradient . . . . . . . . . . 98

FIGS. 8-I2. Reorganization in Pieces of Planaria dorotocephala from Different Body Levels in Relation to Presence or Absence of Head . . . . . IoI

FIGS. I3-I5. Axial Gradients in Development of the Central Nervous System . . . . . . . Iog

Figs. 16, i7. Transverse Sections of Chick Embryo at Different Levels, to Indicate Developmental Gradients

FIg. I8. Sensory Cells of Sea-Anemone Tentacle and Their Outgrowths . . . . . . . . II2

FIGS. I9-22. Planaria dorotocephala: Reconstitution of Pieces from Different Levels . . . . . I22

FIGs. 23-29. Tubularia: Reconstitution of Short Pieces . I24

FIgs. 30-36. Partial Structures from Short Pieces of Planaria I25

FIG. $\quad 37$. Regions of High Susceptibility in Early Larval Stage of Chaetopterus, a Polychaete Annelid

FIG. 38. Unsegmented Egg of Frog, Viewed from Apical Pole, Showing Differential Susceptibility . 
Fig. 39. Stages of Disintegration of Frog Embryo During Late Cleavage . . . . . . . . 137

FIgs. 40-44. Embryonic Segmentation of the Central Nervous System of Vertebrates

Fig. 45. Motor Cell of Ventral Horn of Spinal Cord from Human Embryo . . . . . . . ${ }_{158}$

Fig. 46. Pyramidal Neuron of Cerebral Cortex of Rabbit $\quad{ }_{5}^{8}$

Fig. 47. Neuron with Short, Branching Axon, from Cerebral Cortex . . . . . . . . ${ }_{15} 8$

FIg. 48. Purkinje Cell from Human Cerebellum . . I60

FIG. 49. Fusiform Cell from Anterior corpus quadrigeminum of Rabbit . . . . . . . . . I60

Fig. 50. Monopolar Neuron from Mesencephalic Nucleus of Fifth Cranial Nerve . . . . . I60

FIG. 5r. Neuron of Smaller Gustatory Type from Carp I60

FIg. 52. Neuron from Cephalic Ganglion of Crab, Carcinus maenas . . . . . . . . $\mathrm{I}_{6}$

FIg. 53. Neuron from Ventral Cord of Earthworm . I62

FIG. 54. Development of the Pyramidal Neuron . . 163

FIG. 55. Purkinje Cell from Cerebellum of New-born Infant . . . . . . . . . . . $\mathrm{I}_{3}$

FIg. 5 56. Two Views, Taken Fifty Minutes Apart, of Same Nerve Fiber, Growing from Group of Embryonic Spinal Cord Cells into the Lymph of Tissue Culture . . . . . 168

FIg. 57. Diagram Illustrating Kappers' Conception of the Action of the Bioelectric Current on the Neuroblast

FIGS. 58, 59. Diagrams Illustrating Electrical Polarization of a Cell by Bioelectric Currents . . . . 183

FIgs. 60, 6r. Transverse Sections of Spinal Cords, Showing Directions of Outgrowths of Early Axons . I86

FIGS. 62-65. Developmental Stages of Neural Tube and Spinal Ganglia in Salamander, Amblystoma punctatum 
Fig. 66. A Cell of the Nervous Reticulum of a Coelenterate . . . . . . . . . 203

Fig. 67. Side View of the Body of the Ctenophore, Pleurobrachia . . . . . . . . . 213

Fig. 68. An Epithelio-Mruscle Cell ("Neuro-Muscle Cell") of $H y d r a \quad$. . . . . . . . . . 238

FIG. 69. Diagram of Simple Multicellular Arcs . . . 239

FIG. 70. Diagrammatic Dorsal View of the Upper End of the Spinal Cord of Larval Amblystoma . 253 



\section{CHAPTER I \\ THE PROBLEM OF PATTERN}

Life manifests itself in the form of more or less clearly defined and delimited entities which we call individuals. Biologists are in general agreed that some sort of order, unity, harmony or wholeness as it is variously called, in short, some sort of integrating or ordering factor or principle, effective in both space and time, underlies organic individuality. An adequate conception of physiological integration must afford a basis not only for the spatial localization of parts and for the sequence of events in time in the development of the individual, but also for the functional relations of both nervous and chemical character between parts. And particularly it must account for the origin and development of the nervous system as the organ of integration par cxcellence. The present book is an attempt to show at least that the nervous system does not represent a new integration imposed in some way upon primitive protoplasm, but rather a product, a result of the primary integrating factors which make the organism an orderly whole.

\section{PATTERN AND MATERIAL}

Like any other orderly entity the organic individual presents two great problems, the problem of pattern and the problem of material, and it is with the first of these, the problem of pattern, that we are here chiefly 
concerned. The crystal is not a purely fortuitous aggre. gation of molecules, the house is not simply a pile of lumber, bricks or stone, mortar, etc., nor is the organism simply a kaleidoscopic mixture of substances. In all these types of individuality the relations of the constituent materials are ordered and controlled in accordance with some sort of pattern. Pattern then, in the broad sense, is the particular kind of order, unity, or wholeness which constitutes the entity or individual, whether it be crystal, house, or organism. Moreover, in the organism as elsewhere, the distinction between pattern and material is clearly indicated by the fact that very similar patterns may exist in very different materials. For example, certain hydroids resemble very closely certain plants as regards the order of budding and the growth relations of branches. As regards general axial relations certain bilaterally symmetrical plants, e.g., Marchantia, are very similar to certain bilaterally symmetrical animals, such as Planaria, and very similar radiate arrangements of parts are found in many different plants and animals. Again, the so-called law of anteroposterior development calls attention to a feature of developmental pattern common to many widely different organisms, and finally the receptor-conductor-effector pattern in some form, ranging from a momentary relation in which the three components are completely interchangeable, to the reflex arc associated with a permanent nervous structure, is apparently a physiological pattern characteristic of living protoplasm in general in relation to an external environment.

Pattern develops in or is imposed upon material of some sort, and the problem of the constitution of this 
material is the other great problem involved in individuality. In the case of the organism this problem includes the physicochemical constitution of protoplasm in the broadest sense. This problem of material resolves itself again at each step into new problems of pattern and material. For example, the cell is the material of which multicellular organisms are composed, but the cell involves a problem of pattern and of material. The cell consists of protoplasm, and the two problems of pattern and material exist for protoplasm as well as for the multicellular organism and the cell, and not only for protoplasm, but for each colloid particle, each proteid, each molecule, and, if we conceive the term "material" broadly, each atom. This is merely a statement of the fact that entities or individuals of certain kinds or degree of complexity, each with its own pattern and material, may be integrated to form more complex individualities which again may serve as material for a new integration and so on. The problem with which we are here primarily concerned is the problem of the nature and origin of the pattern which constitutes the organism as a whole, whether it consists of one cell or many, in short, the problem of organismic ${ }^{\mathrm{r}}$ pattern. While pattern constitutes the basis for an ordering, an integration of the material, it must necessarily be of a sort that is possible in the material. Certain types of building, mechanically impossible in

IIn view of the fact that the word "organism," which implies the existence of a unity and order in the entity so designated, is universally accepted and employed, the word "organismic" is not only biologically and etymologically justified but fills a need which is becoming more and more apparent. The word was used by Rhumbler some fifteen years ago (I9O5). Ritter (I9I9) has used the word "organismal" with a somewhat different connotation. 
wooden material, become possible when the materials are steel or concrete. Protoplasm in consequence of its physicochemical constitution represents a certain kind of material in which certain kinds of pattern are possible, and organismic pattern must lie within the range of possibilities determined by this constitution.

As regards the most minute and presumably the simplest organisms, e.g., certain bacteria and ultramicroscopic forms, if such exist, our knowledge is at present insufficient to permit us even to say with certainty whether they are cells in the ordinary sense of the word, and concerning their pattern as organisms we are almost completely ignorant, but it is at least certain that in the bacteria orderly differences between surface and interior exist. The single cell is of course primarily an organism, though it may as a part of a multicellular organism become specialized and lose to a greater or less degree its capacity for independent existence. And, finally, the multicellular form represents a still more complex order and integration.

\section{ORGANISMIC PATTERN}

The fundamental fact for present purposes is that the organism, as the term implies, represents an order and unity of some sort. Concerning the character of this order in the simplest living forms we know practically nothing, but as regards all other organisms we may say that this order expresses itself in the appearance of more or less definite spatial morphological and functional relations and in a more or less definite and orderly behavior of the whole. It is necessary before going farther to inquire how the organismic pattern or order 
differs from the non-organismic. In the first place the organismic pattern is fundamentally a molar, not a molecular or atomic pattern, for it involves regions consisting of many molecules usually of many different kinds. The pattern of protoplasm is apparently submolecular, molecular, and colloidal, but the organism involves something more, viz., an "organization" of the protoplasm into different molar regions of different constitution or structure. Since these different regions or organs are dynamically active and yet the organism is an orderly whole, physicochemical relations of some sort must exist between the different regions. These relations again are not interatomic or intermolecular, like the relations of ordinary chemical reactions, nor are they like the relations between colloid particles. They are inter-regional relations involving distances of much greater order of magnitude than any of the relations in purely protoplasmic pattern, so that they apparently involve some sort of action at a distance or some means of communication.

In short the organismic pattern is of a very different order of magnitude from the protoplasmic pattern, involving molar dimensions far beyond those of any protoplasmic constituent. It is a differentiation and integration of different regions of protoplasm or of different cells or cell groups and it evidently cannot be interpreted in the same terms as protoplasmic pattern. It may exist within the limits of a single cell or it may involve millions of cells: it may be evanescent, temporary, or readily modified by change in external conditions, or it may be persistent to a high degree, but in all cases it represents a pattern of a different order of 
magnitude from the protoplasmic pattern, and therefore involves integrating and ordering factors which must be different, at least in degree, from those concerned in protoplasmic integration.

Manifestly an adequate conception of the nature of organismic integration and pattern is essential to the progress of biology, because all except the most superficial knowledge of the organism as a whole must be based upon such a conception. Without it the data of biological research have a significance in relation to the living organism somewhat like that of the housewrecker's stock in trade to the house.

\section{THE ORIGIN OF ORGANISMIC PATTERN}

In order to find a point of departure for further attack on the problem it is necessary to consider first whether organismic pattern is inherent in protoplasm or imposed upon it from without. In other words, does organismic pattern develop, so to speak, spontaneously out of protoplasm or the cell, or is it in some sense a response to environmental factors?

Any extended historical consideration of the problem of the origin of pattern or integration in organisms is impossible at this time, but it may be pointed out that the preformists, and notably among them Weismann, have simply assumed as inherent in protoplasm such integration and pattern as seemed to be necessary and have described it in terms of hypothetical entities of some sort. Essentially the same point of view persists among those who postulate a definite, persistent morphological pattern in the chromosome (Morgan, I9I9, chap. xix). Preformistic theory offers us nothing but 
description and statement of problems in certain terms; moreover, the implications of such theory are vitalistic rather than mechanistic.

On the other hand, the epigenetic school in attempting to derive organismic pattern from the relations between living protoplasm and its environment has found it difficult to account for the definiteness and constancy of the organismic unity and order and has failed thus far to formulate any adequate conception of the epigenetic origin and development of organismic pattern. And finally, the vitalist recognizes and states the problem clearly, but attempts to solve it by postulating the existence of a non-mechanistic integrating and ordering principle peculiar to the organism. That is to say, he merely substitutes a metaphysical problem for the scientific problem. In view of the fact that mechanistic possibilities as regards patterns in organisms are by no means exhausted the vitalistic assumptions are not at present justified as anything more than matters of opinion. Preformistic theory can do no more than state the problem in certain terms, and epigenetic theory has not progressed very far toward a solution. In view of this situation it seems necessary as the first step in an attack upon the problem to undertake some further analysis in the attempt at a new formulation.

The organism represents an order and unity in protoplasm which is related at every point to the external world. The development and evolution of organismic integration are essentially the evolution of mechanisms and methods of response and adjustment to environmental conditions. If organismic pattern were 
inherent in protoplasm and a direct result of protoplasmic constitution we should expect it to show a close relation to the differences in constitution, e.g., some such relation as that which exists between constitution and crystalline form in the different haemoglobins (Reichert and Brown, I909). While it is true that no two species or organisms and even no two individuals are alike in all details, yet the more general features of organismic pattern are apparently to a large extent independent of specific differences in protoplasmic constitution. As regards the general plan of spatial arrangement of parts and developmental sequence we find three general types of organismic pattern of very wide occurrence, the completely radiate, in which the pattern is referable to a center, the axiate or polar, in which it is referable to a line, and the bilateral, in which it is referable to a plane. At least most organisms show some one or some combination of more than one of these patterns. Physiologically the most general feature of organismic pattern appears to be that of dominance and subordination, of controlling and being controlled.

The attempts to discover terms in which a preformistic conception of organismic pattern might be formulated have been largely speculative and without satisfactory results. For example, the conception of organismic pattern in purely molecular terms, i.e., of the organism as crystalloid, either as a whole or as a congeries of minute crystals similarly oriented, not only fails to account for the facts, but finds its basis in remote and apparently very superficial analogies, and certain other suggestions along stereochemical lines are purely speculative. The assumption of some other undetermined 
basis of arrangement and order resulting from the interaction of the hypothetical protoplasmic entities, determinants, pangenes, or whatever we choose to call them, is not only more extremely speculative but fails completely as a mechanistic hypothesis. To account adequately in any such terms for the uniformity, definiteness, and constancy of organismic pattern requires the further postulation of something very closely resembling the ordering and integrating principle of the vitalists. And finally, even though we invoke the aid of variation and natural selection at every step, as Weismann has done, our increasing knowledge of the facts of adaptation and of the amazing variety and delicacy of regulatory response to environmental conditions present always new difficulties to the preformistic interpretation.

Turning to the other mechanistic alternative, the imposition of organismic pattern upon protoplasm through its relations with the external world, it is clearly necessary for any adequate conception first, that we know something of the fundamental nature of organismic pattern, and second, that we determine, experimentally if possible, how such pattern originates in the relations between protoplasm and its environment. One of the most striking aspects of organismic pattern, to which attention has already been called (see p. 8), viz., the similarity as regards various of the more general features in different protoplasms indicates that such pattern, at least as regards these features, cannot be very closely associated with the specific differences in the protoplasms of different species or groups and suggests the possibility that it may be primarily quantitative in 
character, that is to say, that such similarities of pattern may result from the existence of similar quantitative situations and relations in protoplasms which are specifically very different in constitution. But in spite of the fact that many different lines of evidence point toward such a conception, and although many authors have called attention to the quan titative character of various aspects of organismic pattern, the distinction between pattern and material and between the quantitative and the specific or qualitative aspects of protoplasmic and cellular behavior has often not been clearly drawn. Particularly is this true of late years, during which chemical correlation, internal secretions, hormones, i.e., the production, transportation and effect in the organism of various substances, supposed or known to be specific in their constitution and action, has received so much attention that certain other aspects of life have been to a large extent ignored, as regards their relation to integration or pattern in the organism. Nevertheless we must at least raise the question, To what extent is physiological integration a matter of quantitative relations in protoplasms of different specific constitution?

\section{PHYSIOLOGICAL CORRELATION AS THE MECHANISM OF} INTEGRATION

If the organism is a physicochemical system the mechanism of organismic integration and pattern must consist in the factors included under what we commonly call physiological correlations. Physiological or organismic correlation may be defined as the sum total of the physicochemical relations of organismic type between 
parts in the organism. It is necessary to make the distinction clear between organismic and protoplasmic correlation. I have already pointed out that organismic pattern is of a different order of magnitude from protoplasmic pattern, and if we admit this it is evident that organismic correlation consists in the relations between different molar regions which may be separated by measurable and often very considerable differences. Chemical reaction between two protoplasmic constituents, adsorption upon a colloid particle, the attraction or repulsion between ions or ionized colloid particles, these and similar relations considered individually are factors in protoplasmic rather than organismic correlation, but these same processes occurring in an orderly way in sufficient quantity may become organismic. Organismic correlation is concerned with masses of protoplasm, cells, molar regions and organs, with the production of substances in mass, their transportation over directly measurable and often considerable distances and their action upon regions and organs as wholes, and with the transmission of dynamic changes, also over directly measurable and often considerable distances. The particular physicochemical processes involved in organismic correlation are of course protoplasmic processes, but the mass, the order, and the distances concerned are of a different order of magnitude from those concerned in strictly protoplasmic relations. At the boundary line between organism and protoplasm, if such a line exists even in the simplest living individuals, there may be difficulty in distinguishing between protoplasmic and organismic correlation, but nowhere else is this the case. The house represents a pattern, an 
integration of a different order of magnitude from the patterns of its constituent materials, whether these be lumber, bricks, stone, or cement, and it is the orderly mass arrangement of the material according to a certain plan which constitutes the house. Similarly the organism represents a pattern which involves the orderly arrangement and relation not of protoplasmic constituents but of protoplasmic masses, cells, and organs. In order to answer the question concerning the nature of organismic pattern, and more particularly the possible rôle of quantitative factors in the origin and development of this pattern, we must determine what the different sorts of physiological organismic correlation are, how they originate, and what part they play in organismic integration.

For present purposes it is convenient to group the correlative factors under three heads: the mechanical or contactual, the chemical or transportative, and the dynamic or transmissive, and to inquire concerning the possible rôle in integration of each of these groups.

The mechanical or contactual factors include all those which involve direct mechanical action, pressure, or tension through direct or intermediary contact. While they may play a part in determining size of organs, direction of growth, or flow of fluids, and indirectly, in certain cases, the irritability or metabolic activity of protoplasm, it is difficult to conceive, in view of the fact that the energy of living protoplasm is largely chemical in origin, that they are primary or fundamental factors in physiological integration. Moreover, in order that they may act in a definite orderly way, differences of some sort must already be present between the differ- 
ent regions or parts concerned, or the differences must arise through the direct local action of some external factor. The rôle of these mechanical factors is manifestly secondary and incidental and is limited to organisms in which pattern of some sort has already been established and is in process of development.

Chemical correlation, so called, perhaps more properly transportative correlation, includes the movements and exchanges of substance between the various parts of the organism. The internal secretions, hormones in general, and also the movements and exchanges of nutritive substances, water, electrolytes, and waste products of metabolism, fall under this head. Transportative correlation, in short, includes the material exchanges and movements, the commercial relations, so to speak, within the organism. These transportative factors in organismic correlation are obviously of great importance, and the work of recent years upon the more highly specialized aspects of such correlation, the internal secretions, hormones, etc., has shown us the bewildering variety and complexity of this kind of correlation in the higher animals. Moreover, when we consider that every organ is to some extent and in some way concerned in transportative correlation and that such correlation plays an essential part in the growth, differentiation and maintenance of the various organs, its significance in organismic integration becomes sufficiently obvious.

The question at once arises whether transportative correlation is the primary and fundamental factor in organismic integration. A moment's consideration shows that the existence of definite and orderly transportative correlation implies the existence, first, of 
definite and orderly differences in different regions, and second, of some means of transportation between the regions, parts, or organs concerned. Between regions which are identical in protoplasmic constitution no definite and orderly transportative correlation of the organismic type is possible. Evidently organismic pattern of some sort must exist to some extent before transportative correlation can arise. In short, it is at once evident that, however important transportative correlation may be in the growth, differentiation, function, and maintenance of parts or organs, it cannot constitute the first step in organismic integration, because this first step concerns the origin of the localized differences which make transportative correlation possible. The material exchanges between the constituents of protoplasm involve, of course, electronic, atomic, or molecular transportation, but it is the ordering and localizing of these exchanges with respect to protoplasmic or multicellular masses, regions, and organs that is the characteristic feature of organismic transportative correlation, and this is possible only on the basis of a pattern of a higher order of magnitude than the protoplasmic pattern.

The protoplasmic pattern alone does not seem to afford any mechanism for the origination of this larger pattern, but such a pattern may of course arise through the relation of protoplasm to external factors. When, for example, some portion of the surface of an Amoeba comes in contact with and ingests food, the reaction itself presupposes the existence of an organismic pattern in the Amoeba, but as a result of the reaction a new kind of organismic pattern arises temporarily, i.e., a food 
vacuole appears, and since the processes going on in and about the food vacuole are different from those in the other regions transportative correlation between the vacuole and other parts becomes possible. For the appearance of this particular nutritional pattern in the Amoeba the external factor, the food, is the directly. determining condition, the pattern arising from the reaction of the protoplasm to the food. Similarly, if one portion of the surface of the cell mass is exposed to an adequate oxygen supply while other portions are not so exposed, a regional difference in rate of oxidative activity may arise which may make possible transportative as well as transmissive correlation. Of course organismic pattern in general arises, not in such purely fortuitous manner, but in some very definite and constant way for each form, but there seems to be no escape from the conclusion that transportative correlation is possible only when such pattern is already present, however it may have arisen.

The correlative factors of the third group, the dynamic or transmissive factors, consist in the transmission through protoplasm or along limiting surfaces of a dynamic change of some sort which is initiated in some particular region. While these factors may involve transportation of electrons or ions from one point to another, they differ from the transportative factors in that the correlative action of one part upon another is accomplished, not by the mass transportation of substance from one to the other, but by the passage of an energetic change which we call excitation or of some dynamic effect of excitation. Capacity for transmission of excitation is, so far as we know, characteristic 
to some degree of all living protoplasm, except perhaps in certain quiescent conditions; moreover, protoplasmic transmission may occur from one region to another, even though no differences, either qualitative or quantitative, existed between the two regions before the excitation of one of them by some external factor, i.e., the excitation-transmission pattern may arise, at least momentarily, through the excitation itself. The nature of the process of excitation and transmission is of course dependent upon protoplasmic pattern, but there is no reason for believing that this protoplasmic pattern can of itself give rise to local excitation in the absence of any external action. It appears then that the excitation-transmission pattern is in the final analysis the result of action of an external factor upon a protoplasm.

Since excitation and transmission undoubtedly occur in all protoplasms it is evident that, while the processes concerned may differ in certain respects in different protoplasms, the occurrence of excitation and transmission is independent of the specific constitution of the particular protoplasm concerned and also independent of the nature of the exciting external factor. Viewed as a whole, the condition which we call excitation, however brought about, appears to be essentially an increase in the rate of living, or at least in the fundamental energyliberating processes concerned in the life of any particular protoplasm, and transmission consists in the spread or passage of excitation from one region to another, certain dynamic changes in an excited region being the exciting factor for adjoining regions. The excitationtransmission relation is then an energetic, nonspecific 
or quantitative relation in a protoplasm, and it may therefore be regarded as the most generalized or most primitive organismic relation. This fact is, I believe, of fundamental, though not generally recognized, significance for physiology, and particularly in relation to the problem of organismic pattern.

This consideration of organismic pattern has established certain points. First, organismic pattern is of a higher order of magnitude than protoplasmic pattern and involves the differential action, differentiation, and relation of regions or masses of protoplasm or cells. Second, although in every case a particular protoplasm with its specific constitution and pattern constitutes the substratum upon which organismic pattern arises and develops, there is no evidence that the protoplasmic mechanism is able autonomously, i.e., without the aid of environmental factors, to give rise in the first instance to organismic pattern. Third, organismic pattern, whether mechanical, chemical and transportative, or excitatory and transmissive, may arise, at least temporarily, through the action of external factors upon a protoplasm. Fourth, the excitation-transmission relation being essentially quantitative and nonspecific appears to be the most generalized and most primitive factor in organismic pattern.

\section{ORGANISMIC FATTERN AND THE CHROMOSOMES}

Apparently there is no escape from the conclusion that organismic pattern is not inherent in protoplasm, but arises in the final analysis from the relation of protoplasm to external factors, and it seems also to be true that the excitation-transmission relation is the most generalized and most primitive component in 
organismic pattern. The conclusion that organismic pattern arises in the final analysis through the relation between protoplasm and environment does not of course exclude the possibility that such pattern once established may persist through processes of reproduction, i.e., may be inherited.

Before accepting this conclusion, however, it is necessary to examine briefly certain aspects of the problem of pattern which have not yet been considered. Except perhaps in the simplest organisms, protoplasm exists in the form of cells with differentiation into nucleus and cytoplasm, the nucleus being commonly regarded as the fundamental portion of the cell. The cell itself represents an organismic pattern and is primarily an organism, though it may undergo integration with other cells into the pattern of the multicellular organism. While there is every reason to believe that the factors concerned in the origin of cell pattern are essentially the same as those concerned in the origin of other organismic patterns, we may for the moment accept cell pattern as given and inquire whether the pattern of multicellular organisms is inherent in the cells. The investigations of recent years in the fields of genetics and cytology have led to the postulation of definite chromosome patterns differing for different chromosomes, and many biologists believe that the totality of chromosome patterns in the nucleus of the germ cells of a species represents in some way, and constitutes the basis of, the organismic pattern which appears in the development of the individual.

Cytological investigation, however, has established beyond question the fact that every cell of the indi- 
vidual body possesses normally the full complement of chromosomes, and therefore, supposedly, the complete nuclear pattern of the fertilized egg. Nevertheless different cells of the body become different and different kinds of organismic correlation arise between them. Boveri recognized this fact years ago and admitted that local conditions rather than the nuclear pattern must be responsible as activating factors for the origin of these differences between the cells, but many biologists have either not discussed the question or have failed to recognize the fact that if all the cells are originally alike they cannot of themselves become different. Loeb, in his book The Organism as a Whole, evades the entire problem with the statement that "the egg is the embryo in the rough" and makes no attempt to show how the egg has attained this condition, and Morgan in his recent discussion of the organism as a whole (Morgan, ${ }^{\mathrm{r}}$ I9I9, pp. 24I-46) fails completely to account for the fact that cells, which are originally alike as regards nuclear pattern, become different in a definite and orderly way, and is very evidently helpless before the problem of the organism as a whole. Moreover, even if we maintain that a cytoplasmic pattern is determined in the egg by the nuclear or chromosomal pattern and that the pattern of the multicellular organism results from the distribution of the components of the cytoplasmic pattern of the egg among different cells in development, we have attained no solution but merely a formulation of the problem of organismic pattern in the purely preformistic terms of nuclear pattern, and the problem of nuclear pattern remains. But there is

${ }^{I}$ For this and similar references, see the list on p. 273. 
still another difficulty involved in this conception. If nuclear pattern is the fundamental factor in determining cytoplasmic pattern, and if the cytoplasmic pattern of the egg represents the pattern determined in this way, as this conception apparently assumes, we should expect each cell which arises with a full complement of chromosomes from the egg gradually to approach the cytoplasmic pattern of the egg, even though different cells originally received different components of the egg pattern. In other words, since they all possess the same nuclear pattern the different cells should tend to become more and more alike instead of more and more different. But since they do become more and more different it is necessary to assume either that cytoplasmic pattern once established may continue to exist independently of, or in spite of nuclear pattern, or else that such cytoplasmic pattern may influence and alter nuclear pattern, and either of these assumptions amounts to an abandonment of the original hypothesis of nuclear pattern as the determining factor. In short, it is evident that even when we start with the cell conceived in terms of the theories of nuclear pattern, something more, viz., the action of an external factor, is necessary to account for the pattern of the multicellular organism.

We may then not only reaffirm the conclusion that so far as the evidence goes, organismic pattern does not appear to be inherent in protoplasm, but we may go a step farther and maintain that the pattern of multicellular organisms cannot be inherent in nuclear pattern. Assuming that this conclusion is correct, organismic pattern must originate in the first instance in the relations between protoplasm and its environment, or 
else it must be dependent upon a non-mechanistic principle, as the vitalists maintain.

\section{CONCLUSION}

Leaving out of consideration the purely contactual or mechanical relation as obviously not of fundamental importance in this connection, there remain two chief categories of relation between protoplasm and its environment, the material or chemical and the dynamic or excitatory. These two types of relation appear in all organisms, the one being concerned with nutrition, respiration, excretion, i.e., the material exchange in general, the other with behavior in the broad sense. I have pointed out that the excitation-transmission relation is apparently the most generalized and most primitive factor in organismic pattern (p. I7). Lest this statement be misunderstood it may be pointed out that it does not mean that in the relations between protoplasm and the environment excitation is any more primitive or fundamental than the material relation. Material exchange between protoplasm and environment is of course necessary for the continuance of life in protoplasm, and there can be no doubt that in nature material exchange and excitation are closely associated. I would merely maintain that the excitation-transmission relation, whether it arises in connection with material exchange or through a purely dynamic action of external factors is a more generalized and more primitive factor in the organismic pattern arising on the protoplasmic substratum. Material exchange is primarily a matter of protoplasmic pattern and not necessarily organismic, but the excitation-transmission relation is always 
organismic when it exists. Moreover, it is not primarily the material exchange between protoplasm and environment, but the ordering and controlling of this change in definite ways which constitute the fundamental characteristic of organismic pattern. In short, however it may arise, organismic pattern is not simply the pattern of the material exchange between protoplasm and environment but rather a definite behavior pattern of different regions, cells, or cell groups. The fundamental factor in determining protoplasmic behavior of organismic character is the nonspecific, quantitative factor of excitation, and with excitation transmission is associated. In view of all the facts, then, the logical procedure in attacking the problem of the origin of organismic pattern is to determine whether, or to what extent, excitation and transmission are actually concerned in determining this pattern. And since the data of both ontogeny and phylogeny indicate that organismic pattern once established undergoes progressive complication, it is evident that we must first of all attempt to discover the simplest terms in which organismic pattern appears in nature. The following chapter is devoted to a consideration of this question. 


\section{CHAPTER II}

\section{THE PHYSIOLOGICAL BASIS OF AXIATE PATTERN}

\section{THE PHYSIOLOGICAL GRADIENT}

The cell is primarily an organism, though it may be integrated with other cells to form a part of a multicellular organism. While we have no positive knowledge concerning the origin of cell pattern, the structure of the cell in general suggests that it is primarily what we may call a surface-interior pattern. If this is true, the differentiation of nucleus and cytoplasm from the primitive protoplasm, possessing in some degree the functions of both, must have resulted in the first instance from differences and relations between exterior and interior. Some organisms appear to be even simpler in pattern than the ordinary cell, but most of them are so minute and show so little differentiation of parts that our knowledge of their pattern is very fragmentary. Even in the simplest organisms, however, we should expect to find at least a surface-interior pattern. In the absence of any positive data, further discussion of the origin of cell pattern and of still simpler patterns is of value only in the light of what we can learn concerning other organismic patterns and is therefore postponed (see p. 60).

The surface-interior pattern is primarily a completely radial, spherically symmetrical or centered pattern and, assuming that organismic pattern arises through relation between protoplasm and environment, 
it represents such relation in its most general and primitive form. While some cells and some still simpler organisms may possess this pattern alone, it is certain that most organisms, including all multicellular forms, at least many unicellular forms and probably some of those simpler than cells, possess not only a surfaceinterior pattern but also an axiate or polar pattern and primarily either a radial or bilateral symmetry with asymmetric modifications in various cases. Both the course of organismic evolution from very early stages as well as the development of the individual are based upon this axiate pattern. I have already pointed out (see p. 8) that the general similarities of organismic pattern in different protoplasms suggest that such pattern is, at least as regards its fundamental features, in large measure independent of the differences in the specific constitution of different protoplasms and therefore essentially quantitative, i.e., it apparently represents a primarily quantitative relation in a specific protoplasm. It now becomes necessary to examine the data of observation and experiment with reference to the question of the nature of the axiate pattern.

Many different lines of evidence agree in indicating that axiate pattern in its simplest form is essentially quantitative in character and consists in graded differences in the rate of the fundamental dynamic activities of protoplasm and in the conditions associated with these activities. These graded differences in physiological state have been called axial gradients because, so far as the evidence goes, they are the primary indications of the existence of an axiate pattern (Child, I9I5c). They have also been called metabolic gra- 
dients, not because it is assumed that they are purely or primarily metabolic in character, but because our knowledge of protoplasm in general indicates that metabolism, and more specifically oxidation, is a fundamental factor in life and the chief source of the energy of living organisms and because the data of observation and experiment indicate that differences in the rate of metabolism and particularly of oxidation are characteristic features of these gradients. Protoplasm is a system in which the chemical reactions of metabolism are so intimately associated with other factors, e.g., colloid dispersion, active mass of enzymes, permeability of limiting surfaces, electrolyte, and water content, etc., that to attempt to distinguish one particular factor rather than another as primary is at present impossible. The axial gradients are not simply oxidative or metabolic gradients but gradients in the general physiological state of the particular protoplasm concerned, and in this physiological state oxidation and associated with it other metabolic reactions are important factors. The term "metabolic gradient" as used in this connection means only that the metabolic factor is a characteristic feature of the gradients. The term "physiological gradient," which avoids all specific implications, might be substituted for it.

In the development and differentiation of the axiate pattern the apical region or head of the organism arises from the region of greatest activity or highest metabolic or oxidative rate, the "high end" of the major or polar gradient, and other organs at different levels of the gradient. The simplest forms of radial symmetry in axiate organisms in which there is no differentiation 
between different radii are in reality nothing more than apico-basal or polar pattern, together with a surfaceinterior pattern. In the more complex forms of radial symmetry a number of radial axes or gradients arise, and the radially arranged parts differentiate in relation to these. In bilateral patterns there is not only a gradient along the longitudinal or polar axis, but bilateral gradients are also present. In at least most bilateral invertebrates the high region of these bilateral gradients is apparently represented by the median ventral region and in the vertebrates by the median dorsal region. The evidence indicates that the chief axial gradients appear primarily in the superficial regions of the cell or multicellular body. In many of the simpler organisms, e.g., many Protozoa (Child, 1914 $b$ ) and plant cells, they are present throughout life only or chiefly in the superficial regions, but where definite localized internal organs with an axiate pattern exist these also show axial gradients in all cases examined, though these organ gradients do not necessarily coincide in direction with the primary gradients. As was pointed out nearly five years ago (Child, I9I5c, pp. 54, 60) the primary gradient or gradients may persist throughout life, but do not necessarily do so. They may undergo modification and complication in various ways during the course of development: the originally quantitative relations may become qualitative, new gradients may arise in certain parts or organs, in some cases pre-existing gradients may be broken up, obliterated, or reversed, one kind of axiate pattern may be replaced by another, and so on. In all cases, however, the sequence of events is definite and orderly, and many features of it can 
already be interpreted in physiological terms, and usually the pattern even in the fully developed organism shows a very definite relation to the primary gradients.

The evidence for the existence of metabolic or physiological axial gradients is varied and extensive and only a brief summary is possible here. The evidence, as it stood five years ago, was briefly discussed in the book Individuality in Organisms, but since that book was written other methods of investigation have been developed and many new data obtained, so that a review of the subject is necessary at this time.

\section{THE EVIDENCE FROM STRUCTURE AND DEVELOPMENT}

First of all many eggs and embryos show an apicobasal gradient in protoplasmic structure and content, e.g., the gradient in yolk accumulation in many animal eggs and embryos, and the gradient in protoplasmic density and vacuolation in many plant embryos, and in the vegetative axes of many of the simpler plants. A gradient in the rate of cell division, growth, and differentiation in relation first of all to the primary of apicobasal axis, later in relation to other axes, is a very general feature of at least the earlier stages of development. This gradient appears first in the rate of cell division and size of cells along the apico-basal axis in a large proportion of both animal and plant eggs and in many plant axes, as a gradient in rate of division and cell size from the growing tip basipetally. It also appears in the progress of morphogenesis and differentiation along the axes, particularly in animals, although in plants, except for the fact that the growing tip itself remains embryonic, the course of differentiation is also 
in general basipetal. Moreover, in bilateral animals and plants a similar bilateral developmental gradient appears more or less clearly. In at least most bilateral invertebrates differentiation of the body wall and its organs progresses from the median ventral region laterally and dorsally and in vertebrates from the median dorsal region laterally and ventrally as a moment's examination of the chick embryo shows. The so-called law of anteroposterior development is merely a recognition of the existence of this developmental gradient in the longitudinal axis and it is, moreover, only a partial statement of what might be called the law of axiate development. We also find evidences of the existence of a polar axial gradient in the rate of regulatory development, the positions and proportions of organs in isolated pieces from different levels of the body in various forms (Child, г907 $b, c$, г1 1 i; Hyman, I9I6). ${ }^{\mathbf{x}}$

\section{THE EVIDENCE FROM SUSCEPTIBILITY}

The existence of these gradients is perhaps most readily demonstrated through the susceptibility of organisms to the action of various external agents. It has been determined experimentally for many species

${ }^{x}$ Figures of these structural and developmental gradients are given in Individuality in Organisms. Figures showing gradients in cell size, rate of division, and in some cases in protoplasmic structure are as follows: cleavage stages of the frog's egg, Figs. Io, II; early developmental stages of plants, Figs. 18, 19; growing tips and axes of plants, Figs. 20-36; adventitious buds in plants, Figs. 37-39. In chaps. iv and $\mathrm{v}$ of the Individuality some of the evidence for the existence of the gradients from regulatory development and its experimental modifications is given. See also Figs. I3-I7, of flat worm and chick on pp. Io9 and II I below. 
of animals, including all the chief phyla and many of the smaller groups and nearly a hundred species of algae among plants, that axial gradients in susceptibility to the action of at least a wide range of external agents exist. The agents used in these experiments include cyanides, many anesthetics, such as alcohol, ether, chloroform, chloretone, some of the urethanes, etc., carbon dioxide, various acids, alkalies, neutral salts, certain alkaloids, vital dyes and physical conditions, such as extremes of temperature and the negative factor lack of oxygen. ${ }^{\mathbf{I}}$ Potassium cyanide has been much used in these experiments first because very low concentrations are effective and second because it has been found by a large number of investigators to be a powerful inhibitor of protoplasmic oxidation and susceptibility to cyanide may therefore be regarded as to some extent a comparative measure or indicator of rate of oxidation in the parts, organs, or individuals or a particular species. ${ }^{2}$

I The data on susceptibility as determined by death and disintegration of cells or tissues, so far as they have been published, appear in the following papers: algae (Child, IgI $6 c, e$, I91 $a, b$, I919e); Protozoa (Child, I9I4b; Hyman, I9I7); coelenterates (Child, I9I8, I919b; Child and Hyman, I9I9); ctenophores (Child, I9I7c); Planaria (Child,

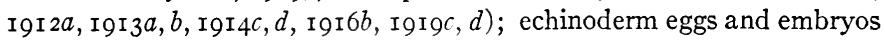
(Child, I9I5a, I916a); annelids (Child, I9I $d$; Hyman, I9I6); Amphibia (Bellamy, I9I9); miscellaneous (Child, I9 $44 a$, I9 I 5C, pp. 50-62). Further data on susceptibility gradients in Protozoa, ctenophores, Hydrozoa, flatworms, echinoderms, annelids, fishes and Amphibia, and the chick embryo are not yet published.

${ }^{2}$ There has been much discussion concerning the nature of the action of cyanides on living protoplasm. Some authors have believed that they act essentially as reducing agents and so retard or prevent the metabolic oxidations. Others hold that they inactivate oxidizing enzymes and still others that their action is primarily on the state of the colloids. The literature of the subject has recently been discussed 
Comparison of the data on susceptibility with data obtained by other methods has shown that a general nonspecific relation between susceptibility and physiological condition exists, at least in the simple organisms and early developmental stages, where the differentiation of organs is not so far advanced that a more or less specific action of particular agents or particular organs is involved. This relation is as follows: to a certain range of concentrations or intensities of the agents used, which is experimentally determined to be above the limit of acclimation or tolerance for the particular species examined, susceptibility varies directly with, though not necessarily proportionally to, the general physiological activity of the protoplasm. To a certain

by Hyman (I9I9). Notwithstanding the differences of opinion concerning the way in which the effects are produced, and it is not improbable that all the views are correct, practically all investigators agree that cyanides decrease intracellular respiration to a marked extent, except in very low concentrations, which may produce some acceleration. As regards Planaria, for example, Allen (IgIg) and Hyman (I9I9) have shown that KNC decreases oxygen consumption 80-90 per cent and that this decrease is reversible. I have found that it also decreases $\mathrm{CO}_{2}$ production and its action is to some extent additive to that of lack of oxygen (Child, I9I9c). Lund (I9I8) has reported that $\mathrm{KNC}$ does not decrease oxygen consumption in Paramecium, but his data are not conclusive, because the concentrations used by him are highly alkaline and produce extreme stimulation and intense motor activity, often continuing for hours, before the inhibiting action of the cyanide appears. Since he failed to reduce the alkalinity to a point approximately that of the normal environment of Paramecium, at which the solutions produce no stimulation, the total oxygen consumption observed by him does not represent the action of the cyanide alone, but includes the stimulating effect of the alkali. There is then at present no reason to alter the conclusion that susceptibility to cyanides is to some extent a measure of physiological condition, so far as intracellular respiration is concerned, 
lower range of concentrations or intensities, also experimentally determined for each species, the rate and degree of acclimation or acquirement of tolerance varies directly with, though not necessarily proportionally to, the general physiological activity of the protoplasm, and the rate and degree of recovery after temporary exposure to the action of the agent also varies in the same way. The metabolic reactions and particularly the oxidations are important factors in the general physiological activity of protoplasm, and many lines of evidence show that susceptibility may be used, within certain limits and with certain precautions, as a rough comparative measure or indicator of the rate of oxidation. This of course does not mean that all the agents used in determining susceptibility act directly on the oxidations. Undoubtedly different agents act in very different ways upon living protoplasm, but the general nonspecific character of the susceptibility gradients demonstrated with many different agents indicates the interrelation of different processes and conditions in the protoplasmic system. The difference between a region of high and one of low rate of oxidation unquestionably involves not simply the oxidative reactions but many other factors, e.g., colloid dispersion, permeability of limiting surfaces, active mass of enzymes, etc., and within physiological limits change in the physiological state involves changes in all these and doubtless other factors. Apparently the general relation between susceptibility and physiological state is primarily an expression of the fact that the dynamic equilibrium of the more active state is, on the one hand, more readily upset by any extreme external action than that of the 
less active state and, on the other, undergoes more rapid and more complete recovery from temporary alteration. In short, so far as susceptibility is nonspecific and quantitative, it is apparently an indicator of the quantitative aspects of physiological state in protoplasm. Susceptibility to the higher, directly lethal concentrations and intensities can be determined by survival time, and in many cases loss of motor activity, swelling or shrinkage of cells, or other changes preceding death can be used as a check on survival time. To lower concentrations, which are not directly lethal, susceptibility can be determined by the degree of inhibition, acclimation, or recovery in growth, development, motor activity, etc., in different body regions or individuals.

The data on susceptibility have demonstrated the existence in a large number of organisms of definite susceptibility gradients as a characteristic, and as the earliest distinguishable features of axiate pattern, not only for the organism as a whole but for various axiate organs and parts. In all cases the differences in susceptibility correspond to differences in structure, rate of growth, development, and differentiation, and in certain cases it has been possible to show that susceptibility gradients correspond to gradients in oxygen consumption and carbon dioxide production.

In various cases it has been possible to follow the apico-basal or anteroposterior gradient from the egg and to show the modifications which occur during the course of development and the appearance of new gradients in particular organs, or in connection with agamic reproductive processes. In the simpler organisms the susceptibility gradients are wholly or largely 
superficial. In the ciliate infusoria, for example, axiation and morphological differentiation are, at least to a very large extent, confined to the ectoplasm, and in the forms which have been examined the ectoplasm alone shows a gradient in susceptibility (Child, I9I4b). As regards at least many plant cells the same is also true. In many of the monosiphonous algae with elongated cells the apico-basal susceptibility gradient is very distinct within the limits of a single cell (Child, I9I $6 c, e, \operatorname{Igr} 7 a, b, \operatorname{I920} a)$ as well as in the axis as a whole. Modifications with advancing age, cessation of growth, budding, etc., appear very clearly in the changes in susceptibility.

The symmetry gradients have been demonstrated in various animals as susceptibility gradients. In certain siphonophores, for example, in which the nectomedusa is bilaterally symmetrical in form, the body ectoderm shows a marked bilateralsusceptibility gradient In the developmental stages of the sea urchin (Child, I9I6a) the symmetry gradients have been observed. In certain polychaete annelid larvae the susceptibility to lethal concentrations has been found to decrease from the median ventral region laterally and dorsally, and in various fishes (Hyman) and Amphibia (Bellamy, I9I9) it decreases laterally and ventrally from the median dorsal region.

In some animals the primary gradients persist throughout life, while in others the original gradients may completely disappear during development. In the development of the hydroid, for example, a very distinct gradient appears in the unfertilized egg and cleavage stages, and the planula shows a well-marked apico-basal 
gradient. But as the time of attachment of the planula approaches, this gradient gradually becomes less and less distinct, and the planula attaches itself by the end which was originally apical, a fact which has long been known. After attachment the original gradient disappears completely, a new gradient in the opposite direction arises at the opposite end, and the first hydranth arises from the high end of this new gradient. In other words, the phenomenon which Loeb and others have called heteromorphosis is a normal feature of hydroid development. Again, in the polyclad Turbellaria the early stages of development show a very distinct anteroposterior susceptibility gradient, the head arising from the most susceptible region, but in the later stages, so far as they have been examined, a reversal in the susceptibility relations occurs, at least in the superficial regions, and in the adult worms the ectoderm of the head is less susceptible than more posterior levels. These data on hydroids and polyclads have not yet been published in full. In the annelids a second gradient in the opposite direction from the primary anteroposterior gradient appears, at least superficially, in the posterior regions as the result of the development of the posterior growing region (Hyman, I9i6; Child, I9I 7 ). But, whatever the changes, they are definite and orderly and associated with the course of development in each species.

As development and differentiation progress the indications of more or less specific relations between particular regions or organs and particular agents become more frequent. In Planaria dorotocephala, for example, we find that the lateral margins of the body 
are more susceptible superficially than in the median regions to alkalies, while in neutral and acid agents there is little difference between median and lateral or the median ventral region is slightly more susceptible than dorsal and lateral. With methylene blue of certain concentrations Mr. McArthur has found that susceptibility of the median ventral region is distinctly greater than that of dorsal and lateral regions. The early embryonic stages of Planaria have not been available for work on susceptibility, but the data on other flatworms and various other bilateral invertebrates indicate that primarily the susceptibility of the median ventral region is higher than that of dorsal and lateral regions and the outgrowth of tissue from cut surfaces in pieces of adult Planaria suggests a greater parenchymal activity in the median ventral region. In the previously published data on the gradients in Planaria (Child, I91 $3 b$ ) it was noted that the relations as regards median, lateral, and dorsal were not entirely clear. As regards the alimentary tract, various facts indicate that in the adult the region of greatest activity and highest susceptibility is in the middle of the body about the base of the pharynx and that a gradient of decreasing activity extends in both directions from this region, but since the alimentary tract is an internal organ and is not readily separable from other parts it is difficult to obtain conclusive evidence on this point. The anteroposterior gradient of ectoderm and body wall persists throughout life, but with the appearance of new zooids at the posterior end new gradients arise in that region, or, more strictly speaking, the original gradient undergoes modification. Apparently the primary 
embryonic relations have undergone more or less alteration, even in Planaria.

With the appearance of more or less specific relations between particular regions or organs and particular agents, the value of the susceptibility method as a means of distinguishing general quantitative differences and relations is of course greatly decreased. In the higher animals apparent specificity of relation between particular organs and particular agents is much more evident than in lower forms and becomes increasingly complex with the progress of differentiation, but even in these forms the general nonspecific susceptibility relations appear in the earlier developmental stages, at least in all forms examined.

While caution is always necessary in interpreting the data of susceptibility in nonspecific quantitative terms it has become more and more evident as the data have accumulated that general nonspecific susceptibility relations do exist, particularly in the simpler organisms and in the earlier stages of development, and that they are indications of fundamental physiological features of organismic pattern.

It has also been possible to control and modify development in definite predictable ways through the differential susceptibility of different levels of the axial gradients.' Such modifications consist in differential inhibition, differential acceleration, differential acclimation and differential recovery, each representing a definite teratological type.

In cases of differential inhibition with respect to a gradient the degree of inhibition varies directly with the susceptibility and activity of different levels. The 
region of greatest activity is most inhibited and less active regions are less inhibited. In the apico-basal or anteroposterior axis, for example, the degree of inhibition is greatest in the apical or head region and decreases basipetally or posteriorly, consequently the positions and proportions of parts are altered in a definite way, the apical or head region being relatively smaller and the basal or posterior regions relatively larger than in the normal form. Microcephaly, for example, is a characteristic result of differential inhibition along the polar axis. In differential acceleration the alterations of proportion are in the opposite direction, and megacephalic forms result. In differential acclimation and recovery growth or development is first inhibited to some extent, but the more active levels of a gradient acclimate or recover more readily and more completely than the less active, so that in these cases also growth or development is finally relatively more rapid or greater in amount apically or anteriorly than basally or posteriorly.

Similar modifications also appear with respect to the symmetry gradients. In differential inhibition in bilateral forms, for example, median regions are more inhibited, while in differential acceleration they are more accelerated than lateral, and in differential acclimation and recovery median regions are finally less inhibited than lateral.

These various modifications are, as regards their more general features, nonspecific as regards agents, and all except the differential accelerations which require the action of accelerating agents can be produced in some degree by a large number, probably by all agents which inhibit general protoplasmic activity and which 
in lower concentrations or intensities permit at least some degree of acclimation or recovery. Moreover, modifications of the same general type with respect to a particular axial gradient can be produced in widely different organisms, e.g., flatworms, echinoderms, fishes, frogs. In many respects these definite developmental modifications constitute the strongest evidence for the relation between susceptibility and the rate of fundamental activities of living protoplasm. ${ }^{\mathrm{I}}$ And finally, differential susceptibility, as a relation dependent primarily upon quantitative rather than upon specific or qualitative differences in the physiological state or activity of protoplasm, provides a simple and adequate basis for the interpretation of much of the work on experimental teratogeny and many of the teratological forms occurring as "accidents" in nature. The cases of cyclopia and microcephaly in fishes, experimentally produced by Stockard (1907, I909, I910, I9II, etc.), are essentially similar to the inhibited types of head in Planaria (Child, I9IIa, I9I5c, pp. I05-17) and to the differential inhibitions in the sea urchin (Child, I9r6d) and in Amphibia (Bellamy, r9r9, and further data not yet published). All these cases involve a greater degree of inhibition of apical or anterior and median as compared with basal, posterior, and lateral regions and all are nonspecific in origin, i.e., can be produced by the action of various agents and conditions.

The susceptibility method makes no pretense of being an exact quantitative method of measuring

${ }^{2}$ Data on the control and modification of development through differential susceptibility have appeared as follows: Child (IgI I $a$, г $15 d$, г $917 d)$; Bellamy (г19), and further data on Hydrozoa, echinoderms and Amphibia are still unpublished. 
metabolism or oxidation, nor is it to be regarded as taking the place of any other method of investigating physiological condition or rate of metabolism or oxidation. Its chief value is as a supplement to other methods. In the first place it enables us to demonstrate the existence of certain characteristic, nonspecific regional differences in physiological condition in organisms, which because of their unicellular character, or their small size, or because of the complications introduced by separating different body regions, are not available material for other more direct and more exact methods. Even in these forms, however, the action of external chemical agents is in general from the surface inward, consequently the information given by the susceptibility method concerns, first of all, the superficial regions of the cell or body, but it is possible in many cases to learn something concerning differential susceptibility of internal parts and organs.

Second, by the modification and control of development through differential susceptibility, the method enables us to show that the differences in condition indicated by differences in susceptibility are fundamental factors in organismic pattern. The conclusions concerning the relation between susceptibility and rate of metabolism or oxidation mean no more than that rate of metabolism or oxidation is a factor in the conditions which determine susceptibility, and it is not claimed that this conclusion is universally valid. Undoubtedly, in the more highly differentiated organisms and in more advanced stages of development the qualitative differences in different organs may determine differences in susceptibility which are more or less specific as regards 
both organ and agent, but in the simpler forms and the earlier stages the similarity of the susceptibility gradients in widely different organisms and with a great variety of agents renders their nonspecific character sufficiently clear, and many lines of evidence, both direct and indirect, indicate their relation to rate of metabolism or oxidation. The nature and degree of that relation in each particular case and for each particular agent remains of course to be determined by other methods of investigation. The susceptibility method has served to bring to light certain characteristic features of organismic pattern which have not previously been clearly recognized, viz., the gradients, but conclusions concerning the exact nature of these gradients are possible only on the basis of all the different lines of evidence obtainable, and at present of course cannot be final.

\section{THE EVIDENCE FROM RATE OF PENETRATION}

In many forms, both plants and animals, in which susceptibility gradients exist, corresponding gradients in the rate of penetration of certain substances, particularly the vital dyes neutral red and methylene blue, which have been most extensively used in these experiments, have also been demonstrated. ${ }^{\boldsymbol{x}}$ The existence of these gradients in rate of penetration raises the question whether the susceptibility gradients are not primarily gradients in permeability of the protoplasmic surfaces to the agents used. While there is no doubt that a gradient in permeability is one aspect of the axial gradient, our conception of the relation between per-

${ }^{2}$ Much of this work has been done by Mr. J. W. MacArthur and is not yet published. For some observations on algae see Child (Igrge). 
meability and the gradient must depend very largely upon the terms in which we define permeability. If permeability is dependent only on the physical condition of the limiting surface and independent of chemical activity the axial gradients are manifestly something more than mere permeability gradients. But the protoplasmic limiting surface or membrane is certainly polyphasic in constitution, since it is alive and the seat of more or less chemical activity, and its permeability depends upon its living condition and changes when it dies. Moreover, susceptibility as determined by the higher concentrations and intensities of external agents depends rather upon the destruction or alteration of the limiting surface as a living membrane than upon the passage of the agent through the living membrane into the interior of the cell. The susceptibility gradients can be demonstrated not only by agents to which the protoplasmic surface is highly permeable, e.g., vital dyes, various anesthetics, but by those to which it is highly impermeable, such as mercury and copper salts, and by extremes of temperature and the negative condition lack of oxygen, which do not involve the action of any external chemical agent upon the surface.

Moreover, the phenomena of differential acclimation and recovery in growth and development indicate very clearly that the metabolic activity of protoplasm is a factor in susceptibility. With the advance of our knowledge it becomes increasingly evident that the factors concerned in the permeability of living protoplasm are essentially those concerned in other aspects of life and that permeability is an expression of the physiological state of the plasma membranes. If we 
admit this, differences in permeability are themselves to some extent indicators of differences in physiological state, but it still remains true that susceptibility is not simply a matter of the rate of penetration through the plasma membranes, but rather of the rate of killing or alteration of the membranes and superficial regions of protoplasm by an external agent. Different external agents may and undoubtedly do act chiefly or primarily upon different factors concerned in the maintenance of physiological state, but since these different factors are mutually associated in such maintenance, the general result as regards susceptibility, i.e., the general effect on the physiological state, may be and is the same for at least many different agents. In short susceptibility is within certain limits and in a general way an index of physiological state in protoplasm, and the axial gradients in susceptibility are therefore significant, particularly when their existence is confirmed by other methods as indicating the existence of nonspecific or quantitative differences as the earliest distinguishable features of axiation.

\section{THE EVIDENCE FROM CERTAIN OXIDATION-REDUCTION} REACTIONS

The axial gradients have also been demonstrated in many forms as a differential in the rate and amount of reduction of potassium permanganate by protoplasm. It is a well-known fact that $\mathrm{KMnO}_{4}$ is reduced by protoplasm and the reduced salt appears on or in the protoplasm as a brown or blackish precipitate. All axiate forms examined, including numerous Protozoa, eggs, embryonic and larval stages or adults of the lower 
invertebrates, echinoderms and smaller arthropods and various algae among plants, show gradients in the rate of staining by permanganate corresponding to the gradients demonstrated by susceptibility and other methods. The precipitation of the reduced salt and the appearance of the brown color apparently begin on the external surface of the protoplasm, often within a few seconds after the organisms are brought into the solution, and the differences in rate of precipitation and staining at different levels of an axis are usually very marked. Penetration into the protoplasm usually occurs rather slowly, its rate depending somewhat on concentration, and it is certainly not to any large extent dependent on permeability of living membranes but rather on the killing of the protoplasm from the surface inward.

If the reaction is allowed to continue to completion in excess of permanganate, the whole organism may become opaque black and no gradient is visible, but many small organisms thus stained, e.g., blastulae, hydroid planulae, small monosiphonous algae, can be made more or less transparent after such staining by hardening, clearing, and mounting, and in such cases the gradient in staining appears. Under these conditions the gradient represents a gradient in the total amount of reduction of permanganate of which the protoplasm is capable, and it is highly significant to find that the protoplasm of the apical region of a blastula or an alga axis, for example, is capable of reducing more permanganate than more basal levels. If the organism is first killed by some other agent, e.g., various histological fixing agents, heat, etc., reduction and staining are uniform, or in some cases slight traces of the gradients 
are still present, for a time, but after a few days in alcohol they are completely absent in all cases examined.

Since the chemical reaction concerned here is an oxidation-reduction the rate and amount of reduction of permanganate must be associated in some way with the oxidative activity of protoplasm, the regions of higher rate of oxidation showing a higher rate and greater total amount of reduction. We find that the permanganate gradients correspond with those demonstrated by other methods, i.e., the more active and more susceptible regions reduce permanganate more rapidly and in larger amount than the less active and less susceptible. This method is a very delicate one and I believe of considerable value as a means of determining regional and axial differences in physiological state, particularly in small organisms.

In certain cases the indophenol reaction has been used to demonstrate the existence of axial gradients in blastulae and gastrulae. This reaction is an oxidation which is catalyzed by oxidizing enzymes and which results in the formation of indophenol in the form of a blue precipitate. In living starfish blastulae and gastrulae (Child, I9I5a) a distinct apico-basal color gradient has been observed by this means, the formation of indophenol occurring most rapidly in the cells of the apical region. If the animals are killed by some other agent before the indophenol reaction, the blue color is much less marked and the gradient is absent.

\section{THE EVIDENCE FROM BIOELECTRIC PHENOMENA}

Axial gradients in electric potential have been found to exist by Drs. Bellamy and Hyman in axiate animals 
so far as examined, the region of greatest physiological activity as indicated by other methods showing in general the highest electronegativity through the galvanometer. These data are not yet published. Observations along these lines have been made by Mathews (1903) on hydroids, Waller (1903) on various organisms, and Hyde (I904) on certain vertebrate embryos, and both Mathews and Waller point out the probable relation between electric potential and physiological or metabolic activity. Morgan and Dimon (1904) in a study of electric potential in the earthworm found that in general the two ends were electronegative to middle regions and concluded that the potential differences were not related to physiological polarity. We know now, however, that the earthworm and other annelids develop very early a growing region of high physiological activity at the posterior end (Hyman, I9r6; Child, I9I $7 d$ ) and that the body in later stages shows two gradients in opposite directions. The observations of Morgan and Dimon do not therefore conflict with those of others. More recently Tashiro (I9I7 and earlier papers) on the basis of his work on $\mathrm{CO}_{2}$ production in the nerve fiber has pointed out the probable relations between the electric phenomena and metabolic activity in nerve, and Hyman (I9r8) has also suggested that bioelectric phenomena in general are primarily due to differences in metabolic activity. While differences in potential undoubtedly may arise in organisms from other causes than differences in metabolic or oxidation rate the facts in general indicate that such differences are at least very generally associated with differences in metabolic rate. In any case the axial gradients in potential indicate the 
existence of graded differences in physiological condition along the axes, and in the light of other data there can be little doubt that differences in rate of metabolism, or more particularly oxidation, play at least a very important part in determining the electrical gradients.

THE EVIDENCE FROM OXYGEN CONSUMPTION AND CARBON DIOXIDE PRODUCTION

The direct determination of metabolic activity in different body regions by means of oxygen consumption and $\mathrm{CO}_{2}$ production requires the separation of the regions concerned and therefore introduces various complicating factors. Only in the simpler organisms is it possible to maintain such separated pieces of the body in anything like a normal condition, and even here the operative procedure involves stimulation and may be followed by depression, and cell division and growth begin at the cut surfaces within a few hours after section. Moreover, in organisms with localized and differentiated internal organs the axial gradients do not necessarily run in the same direction in all organs, and a piece of the body from a particular level may represent a low level of the body-wall gradient and a high level as regards certain internal organs, or vice versa. In Planaria, for example, the mouth is near the middle of the body and the activity of the alimentary tract probably decreases from the mouth in both directions, but in the body wall the gradient is from the head posteriorly. If this is true, a piece of the Planaria body from levels near the head represents a high level of the body-wall gradient and a low level of the alimentary tract, while a piece from near the mouth represents 
the reverse condition. Unless we can eliminate one or the other of these gradients the oxygen consumption and $\mathrm{CO}_{2}$ production of such pieces is not likely to give us any very definite information as regards either gradient. In this case, however, we can bring the alimentary tract into a more or less quiescent condition by starvation and then we find that a gradient in oxygen consumption and $\mathrm{CO}_{2}$ production does exist in the body wall, the high end being at the head with a second rise in the region of the posterior zooid. The data along this line are as yet mostly unpublished, but one paper on $\mathrm{CO}_{2}$ production in Planaria has already appeared (Robbins and Child, I920) and work on oxygen consumption has been done by Dr. Hyman with similar results. But the relative amount of alimentary tract tissue in pieces of a given weight taken near the head is less than in pieces taken near the mouth, so that even in animals which have been starved for some time the difference in total oxygen consumption or total $\mathrm{CO}_{2}$ production of pieces from different regions is not strictly comparable with the regional differences in susceptibility of the body wall. Where it has been possible thus far to use these methods we have found that the axial gradients demonstrated by other methods are also gradients in oxygen consumption and $\mathrm{CO}_{2}$ production.

\section{EVIDENCE FOR THE EXISTENCE OF PHYSIOLOGICAL} GRADIENTS IN VARIOUS ORGANS

By one method or another or by several different methods the existence of physiological gradients in various axiate organs or parts of many organisms has been demonstrated, e.g., in various reproductive axes 
and in the "hairs" of algae (Child, I9ı $6 c, e$, I9г $7 a$, г9г9e), in the larger, slow moving flagellum of Noctiluca, in the tentacles of Hydrozoa (Child and Hyman, I9r9; Child I9I9b), in the plate rows of ctenophores (Child, I9I7c), the growing arms of echinoderm larvae, the branchiae and sensory tentacles of various annelids, the growing tail of the ascidian and the amphibian tadpole, etc. Dr. Hyman has found that the embryonic heart of the chick and of the fish represents a susceptibility gradient with the high region at the sinus end. But the most extensive work on the metabolic gradient of any organ is that of Alvarez and his assistants on the vertebrate alimentary tract. ${ }^{\mathrm{I}}$ They have found in the small intestine a gradient in irritability, latent period, tone rhythm, conduction, susceptibility to various drugs, and gradients in at least some of these conditions in the wall of the stomach and colon. Tashiro (I9I 7 and earlier papers) has found a gradient in $\mathrm{CO}_{2}$ production in certain nerves, the direction of functional conduction being down the gradient and in certain of these nerves a susceptibility gradient has been observed (Child, I9I4a).

In an investigation of the respiration of ground nervous tissue C. G. MacArthur and Jones (1917) have found differences in rate of respiration in different parts of the central nervous system which indicate the existence of a gradient in rate of respiration. The rate of respiration is highest in the cerebrum and decreases in the various parts in the following order: cerebellum, midbrain, medulla, corpus callosum, spinal cord nerve. The authors find that gray matter consumes about twice as

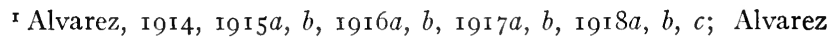
and Starkweather, г9г $8 a, b, c, d, e$, гөг9; Alvarez and Taylor, г9г $7 a, b$. 
much oxygen and produces about one and one-half times as much $\mathrm{CO}_{2}$ as white matter, and some of the differences in respiratory rate in different parts of the nervous system are doubtless due to differences in proportion of white and gray matter. For example, the relatively low rate of the corpus callosum is undoubtedly associated with the fact that it consists of white matter, nerve fibers, rather than cells. But the differences in respiratory rate between cerebrum and cerebellum and between midbrain, medulla, and spinal cord are scarcely to be accounted for in this way. These differences constitute highly significant evidence for the existence of an axial gradient in rate of respiration in the central nervous system. More recently MacArthur and Doisy (I9I9) have demonstrated the existence of a gradient in chemical differentiation in the brain.

All the evidence is in agreement as regards the existence of these physiological axial gradients. Thus far every living physiological axis examined has given evidence of the existence, at least in the earlier developmental stages of such a gradient, and in many cases the experimental methods show the presence of a gradient where structural, or other directly visible indication of its presence are absent. In whatever terms we may finally interpret these gradients, there can be no doubt concerning their existence. They are physiological facts, and their significance for localization, differentiation, and functional relation is already demonstrated. 


\section{CHAPTER III}

THE ORIGIN OF AXIATE PATTERN IN NATURE AND EXPERIMENT

It is a familiar fact that physiological axes persist through certain agamic reproductive processes and are therefore inherited by the individuals resulting from such reproduction, but in at least many eggs the axiate pattern apparently arises de novo during the growth of the egg. Granting that axiate pattern in its simplest form is a gradient pattern, as pointed out, we have at present no grounds for believing that such pattern is inherent in protoplasm, or that it can arise in protoplasm apart from the action of environmental factors. Apparently a differential exposure of the protoplasm to some environmental factor or factors which affect its rate of activity, i.e., which are primarily quantitative rather than specific or qualitative in their action, is necessary for the origination and establishment of an axial gradient in protoplasm. Moreover, the quantitative nonspecific character of the gradient pattern suggests that its origin is to be sought in the quantitative rather than in the qualitative or specific aspects of the relations between protoplasm and environment. In other words the gradients must arise through the differential action of environmental factors which affect primarily the rate of general protoplasmic activity.

THE ORIGIN OF POLARITY

As regards the plants, Winkler (I900b) and Kniep (I907) showed that in certain algae, e.g., various species 
of Fucaceae differential illumination of the two sides of the egg determines the polarity of the plant developing from the egg, though in the absence of light polarity appears and germination occurs, but more slowly. Stahl (1885) showed that in the spore of Equisetum polarity is determined in the same way. Winkler (I900a) also showed that polarity in the alga Bryopsis could be determined by light. In various bilaterally symmetrical plants, such as liverworts, light determines the dorsiventrality and some algae develop a radially symmetrical thallus when the illumination is equal on all sides of the polar axis and are bilateral when the illumination is from one side. In the spermatophytes conditions which determine egg polarity are undoubtedly intraorganismic. The embryo sac shows a definite polarity with respect to surrounding parts and the ovum is attached to one end of the sac. The unattached end of the ovum becomes the apical, the attached end the basal pole of the embryo. What particular factors are concerned in this case is not known.

In the simpler animals we see new polarities arise at cut ends of pieces, e.g., the development of new apical regions and axes from the aboral ends of pieces in various hydroids, the development of heads from posterior cut surfaces in Planaria (see Figs. 30-34, p. I25). In such cases the new axis is always represented by a new gradient and the relation between the new axis and the occurrence of differential exposure is obvious, though whether the wound stimulus, oxygen supply, or some other factor is chiefly concerned in determining the new gradient is not known. Loeb (1892) has maintained that new polarities are determined by gravity in the 
regulatory development of the hydroid Antenmularia antennina, but Morgan (I901) and Stevens (1902, I910), while not disputing Loeb's results, showed that other factors besides gravity were concerned in determining polarity.

In the development of sponges from dissociated tissue cells described by H. V. Wilson (I907, I9II) the polarity of the new individual is determined by some sort of differential between free and attached surfaces of the cell mass, the osculum developing on the free surface. Similarly in the experiments on obliteration of preexisting polarity and the establishment of a new polar axis in hydroids (Child, I9I $5 c$, pp. I42-46) the apical region of the new axis arises in the region of greatest exposure to the environment, but the particular factors chiefly concerned have not been determined. In both these cases, the sponge and the hydroid, the difference in oxygen supply on the exposed surface of the cell mass and the surface in contact suggests a probable factor in determining the new axis.

In the actinian Harenactis the localization of a region of more rapid and more extensive growth by injury is sufficient under certain conditions to determine the position and development of new polarities (Child, I9ıob, I9I5c, Figs. 79-83). The axial gradation results in this case from the fact that the activity of the cells is greatest in the middle region of such an area and decreases toward its borders. In this connection a recent statement of Harrison's concerning the limb rudiment of Amblystoma is of interest. Harrison says: "The limb rudiment may be thus regarded, not as a definite circumscribed area like a stone in a mosaic, 
but as a center of differentiation in which the intensity of the process diminishes as the distance from the center increases, until it passes away into an indifferent region. Many other systems, such as the nose, ear, hypophysis, gills, seem to have the same indefinite boundaries which may even overlap one another" (Harrison, I9I8, p. 456). Evidently Harrison conceives these primordia as gradients in activity in a more or less specialized cellular region of the embryo. Such gradients differ from the general axial gradients of the body only in that they are determined in some way, presumably by intraorganismic correlative conditions in specialized body regions, and are concerned with particular organ complexes instead of with the body as a whole. In still other cases new polarities are apparently determined and localized by slight differences in activity between different cells of a mass. Such differences determine the more or less definite localization of a region of growth in which the activity decreases toward the periphery, and as growth progresses an axial gradient arises. Determination of new polarities in this way apparently occurs in many cases when pieces of naked hydroid stems give rise to multiple stolons, each of which represents a new axis and a new gradient. These multiple polarities have been observed by many investigators, and I have been able to produce them experimentally in hydrozoan planulae by first obliterating the original polarity through differential inhibition. In the origin of adventitious buds from the epidermal cells of the Begonia leaf similar local growth areas with gradients in activity from center toward periphery and from the surface inward are the first indications of the new plant axes (Regel, I876; 
Child, I9I 5 c, Figs. 38,39 ). The localization of the new axis in such cases appears to be largely a matter of slight fortuitous differences in activity in different cells or cell groups, in consequence of which certain cells or groups react more rapidly than others to the experimental conditions.

As regards the animal egg, the evidence is very incomplete but indicates that in at least many forms polarity is determined during the growth period of each egg by differential exposure. In various hydromedusae, for example, the growing oögonia constitute a columnar epithelium, one end of each cell being separated from the exterior only by a layer of very thin flattened cells, while the opposite end is attached and adjoins the radial canal (Figs. I-2). When portions of the ovaries are slightly teased to separate the eggs it is found that the free end of the egg, the end nearest the exterior, represents the high region, and the attached end, the low region of a gradient in susceptibility and permanganate reduction (Fig. 3). A similar gradient appears in developmental stages, and while the absence of good landmarks makes it impossible to demonstrate that the later gradient is identical with the earlier, there can be little doubt that it is. In the sea urchin the oögonium is attached to the wall of the ovary at one small region of its circumference and here also, as Boveri has shown, the free pole becomes the apical pole and represents the high end of a gradient. In Sternaspis an aberrant annelid, a peduncle containing a vascular loop, develops in connection with each growing oögonium, but my observations indicate that the free pole, not the attached pole of the egg, into which the vascular loop enters, 
becomes the apical pole (Fig. 4). In these cases it is not the pole through which nutrition enters, but the unattached pole, which in the medusa is more exposed to external factors and in the sea urchin and Sternapis to the fluids of the ovary which becomes the apical pole. It seems probable that a differential in oxygen supply
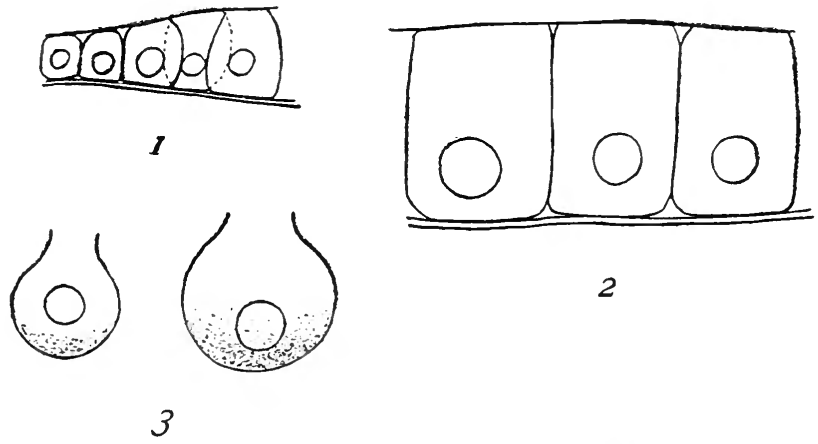

2

Figs. I-3.-Ovarian eggs of jellyfish, Phialidium gregarium; diagrammatic: Fig. I, early growth stages; Fig. 2, later stages; in these stages one surface (the lower in the figure) is separated from the exterior only by thin epithelium and opposite surface is attached and adjoins radial canal; Fig. 3, eggs separated from ovary by teasing, showing region of attachment; shading indicates susceptibility gradient as indicated by disintegration and by permanganate.

and perhaps also in $\mathrm{CO}_{2}$ concentration are chiefly concerned in determining the polarity and the gradient which represents it in these cases.

In the eggs of at least some higher animals, where oxygen as well as nutritive substances reach the egg chiefly or wholly through the blood, the polarity is apparently determined by relation to the blood supply. Bellamy (I9I9) has shown that in the frog's egg polarity apparently develops in definite relation to the vascular 
supply, the apical pigmented pole arising on the arterial, the basal unpigmented pole on the venous side (Fig. 5). In these cases the oxygen supply or the conditions determining rate of respiratory exchange are apparently

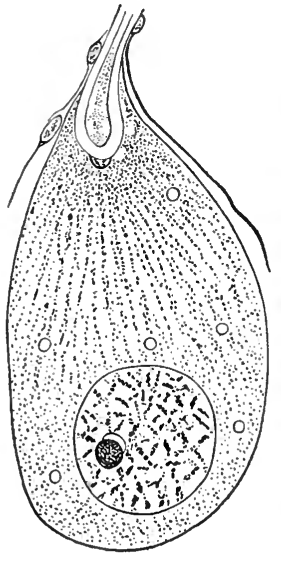

4

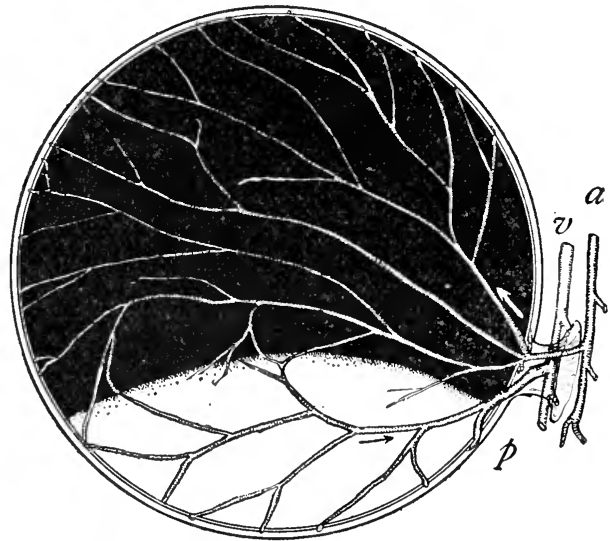

5

FIG. 4.-Growing oöcyte of Sternaspis scutata (from Child, I9I $5 b$ ).

FIG. 5.-Semi-diagrammatic view of ovarian egg of frog, showing distribution of arteries and veins in relation to pigmentation and polarity: $a$, artery, $v$, vein, $p$, pedicle (from Bellamy, I9I9).

the chief factor in determining polarity, the region of most rapid exchange becoming the apical pole.

\section{THE ORIGIN OF SYMMETRY}

Symmetry in animals, like polarity, is primarily indicated by gradations in physiological condition, and although a particular kind of protoplasm may give rise normally to a radial or a bilateral animal, experiment 
shows that for certain forms, at least, the normal symmetry of pattern is not inherent and unchangeable. In the radial anemone Harenactis, for example, bilateral tentacle groupings may arise under certain experimental conditions (Child, I909), and it is possible through differential susceptibility to obliterate bilaterality and produce radial larval forms in the sea urchin (Child, I9r6d) and also in the starfish (unpublished). Moreover, in pieces of Planaria, under experimental conditions which practically obliterate the polar gradient the symmetry gradient may become the polar axis of the new individual (Child, I9 $5 c$, pp. I6 $63-65$ ).

A characteristic feature of radial symmetry is the repetition about a center of parts, each of which is usually axiate and often bilateral in pattern. Such repetition must be largely a matter of the space relations of specialized growth centers. Each growth center involves or dominates a certain area, and only at a certain distance from it can another similar center arise. Thus the number of such growth centers arising on a given circumference depends on the area dominated by each center, which varies with physiological condition, and on the size of the circumference, and can be altered experimentally in many cases. Moreover, as growth in size of the circumference occurs and the distance between the repetitive parts increases, we often find additional new parts arising, e.g., mesenteries and tentacles in many actinians, etc. Such processes are physiologically similar to many forms of agamic reproduction, being essentially reproductions of specialized parts instead of new wholes, resulting from physiological isolation (Child, I9 I 5 c, chaps. iv, v). 
Bilaterality is inherited through many processes of agamic reproduction, e.g., in flatworms and annelids, and may of course also be inherited in many eggs. On the other hand, it may conceivably be determined in some eggs by ovarian conditions, by conditions connected with maturation or with fertilization or perhaps even by conditions arising later in development. For example, the median ventral trunk region in the polychaete annelids arises from the fusion or concrescence of the growing borders of the somatic plate (Child, I9I7 $d$ ), and in the vertebrates from the concrescence of the growing border of the blastoderm or the lips of the blastopore. The higher frequency of cell division and the smaller size of cells in the lateral border of the somatic plate of various annelids suggest that it is a region of high physiological activity, and in certain cases $I$ have found that it is more susceptible to lethal concentrations than other parts of the somatic plate. Hyman has observed that in certain of the fishes the border of the blastoderm becomes a region of high susceptibility as it begins to form the germ ring and to grow down over the yolk (unpublished). As the embryo is formed, this active border undergoes concrescence to form the median dorsal region. In the frog (Bellamy, I9I9) after gastrulation the lips of the blastopore, which are overgrowing the yolk, constitute a region of high susceptibility to lethal concentrations, most marked in the dorsal region. These lips form the median dorsal region of the embryonic body. These facts suggest that the mediolateral gradients in the annelids and in at least the lower vertebrates may be associated to some extent with the differences in physiological con- 
dition between the more exposed and probably more stimulated growing borders of the somatic plate, the blastoderm or the blastopore lips and other adjoining regions. Moreover, many bilateral forms develop characteristic asymmetries during the course of development, e.g., the asymmetry of gasteropod mollusks and the visceral asymmetry of vertebrates, or a welldeveloped bilateral symmetry may give rise in metamorphosis to radial-bilateral pattern of very different kind, as in certain echinoderm groups. Our knowledge concerning the physiological aspects of the origin of symmetry in animals is still very fragmentary, but the earliest indications of the presence of a particular symmetry pattern are gradients in physiological condition, which, so far as the evidence goes, are similar to the polar gradients, and in plants we see the different symmetry patterns arising through differential exposure to the action of external factors. In the light of all the facts we are justified in concluding that even though a particular symmetry pattern may persist through reproduction, i.e., be inherited in a particular case, symmetry like polarity must in the final analysis arise through differential exposure to the action of external factors.

Even if we grant that the differential exposure of the egg of the medusa, the sea urchin, Sternaspis, etc. (see p. 54), determines the polarity and that symmetry may also be determined by relation to environment, it is evident that, except in some of the simpler organisms, the differential exposure of the egg is not fortuitous, but is determined by the hereditary mechanism of the organism. The epithelial arrangement of eggs in the 
medusa gonad, the position of the sea urchin egg in the ovary, the development of the peduncle and the vascular loop in Sternaspis, and the circulatory pattern in the chorion of the frog's egg are all features of the hereditary mechanism of the organism concerned. Even in such cases, then, as well as in cases where the axiate pattern persists through reproduction, the hereditary mechanism is concerned in the origin of the axiate pattern of the new individual. Moreover, even in the case of Fucus, where the determination of polarity by the direction of incident light is apparently wholly fortuitous, the hereditary mechanism, as expressed in the constitution and pattern of the egg protoplasm, determines the occurrence and the nature of the reaction to incident light. In other words, the hereditary constitution of the Fucus egg, as well as the direction of incident light is a factor in the determination of the polarity. There is, in short, no conflict between this physiological conception of the origin of organismic or of axiate pattern and conceptions of heredity. The origin and development of organismic pattern in nature is simply the realization of certain hereditary potentialities of a particular protoplasm in a particular environmental complex, which may itself be determined in large measure, or wholly, by the hereditary mechanism of the protoplasm concerned.

\section{THE PROBLEM OF CELL PATTERN}

In the light of the conclusions reached concerning the nature and origin of axiate pattern, the question of cell pattern, touched upon above (p. 23) requires some further consideration. It was suggested that the cell is 
primarily a surface-interior pattern resulting from exposure of the surface of a mass of protoplasm to the action of external factors. Such an exposure is a differential exposure as regards surface and interior. Both the respiratory exchange and excitation can occur only through the surface; therefore differences must arise between surface and interior, and a more or less definite gradient in such conditions from the surface inward must result. As different organs are localized at different levels of an axial gradient, so the localization and differentiation of the nucleus in the first instance may have resulted from the conditions in the interior of the protoplasmic mass. In fact it is difficult to see how the nucleus as a definite organ could have arisen otherwise. The differences between nucleus and cytoplasm as regards acidity and electric potential, as well as the behavior of nuclei in such specialized cells as spermatozoa, where cytoplasm is practically absent, all suggest that the nucleus is fundamentally an internal cell organ, and if the origin of cell pattern has any relation to environmental factors, the differentiation of the nucleus must have been determined originally by conditions in the interior of a protoplasmic mass. The fact that the nucleus persists from one cell generation to another means merely that the pattern once established is persistent or inherited, although it is difficult to determine to what extent the persistence of the surface-interior conditions is concerned in the persistence of pattern.

Viewed from this standpoint, cell pattern originates in the differential between surface and interior in general and axiate pattern in differentials between different 
parts of the surface of the protoplasmic or cell mass concerned. As regards the axiate pattern, the evidence indicates that the differential is primarily quantitative and involves differences in the rate or degree of fundamental protoplasmic activity, but as regards cell pattern we have at present no means of determining whether the differential was primarily quantitative, though various lines of evidence point in that direction.

THE RELATIONS OF SURFACE-INTERIOR AND AXIATE PATTERN

The presence of an axiate pattern does not necessarily imply the disappearance of a general surfaceinterior pattern, either in the cell or the multicellular organism. All organisms show some kind of surfaceinterior pattern, at least in the superficial regions of the body, and all the facts indicate that in the final analysis such pattern arises through exposure of the surface. The passage of cells to the interior of the embryo by gastrulation is of course a feature of axiate pattern, but conditions in the interior are undoubtedly factors in determining the further differentiation of such cells into the organs of entoderm and mesoderm. Only in some of the simpler animals does the general surface-interior differentiation arise in situ. Formation of entoderm by delamination in all cells of a blastula, for example, appears to be a case in point, and in the Protozoa definite morphological differentiation occurs primarily and chiefly in the ectoplasm. But in the ectoderm of multicellular animals we find numerous evidences of surface-interior pattern ranging from the basal muscular extensions of ectoderm cells in $H y d r a$ 
(Fig. 68, p. 238) to various complicated sensory structures and the early differentiation of the neural tube in the higher animals, and the relation of such features of pattern to exposure of the surface appears obvious. Here as in the case of the axiate pattern, however, the surface-interior relation represents merely the physiological conditions under which the potentialities of the hereditary mechanism of the protoplasm are realized. The fact that the embryo possesses a surface determines certain relations in the protoplasm to this surface and the specific constitution of the protoplasm determines the kind of reaction which occurs, the sort of specialization which develops. If an axiate pattern is also present, physiological conditions are provided for differences in reaction in relation to the surface at different regions or levels of the body. This of course does not mean that exposure to a special external stimulus is necessary for the development of a particular superficial organ. Light, for example, is not necessary for the differentiation in the individual of a photoreceptor or an eye. The surface-interior relation merely determines that the physiological conditions under which the hereditary potentialities of a given protoplasm to produce a photoreceptor are realized, are conditions which arise in development at or near the surface of the developing organism.

The differential exposure of the cell or cell mass to the action of environmental factors is only the first step in the establishment of the gradient. Admitting that this differential exposure determines a higher rate of activity in some region, we may expect, since living protoplasm is irritable and since increased activity in 
one region serves to some extent to excite adjoining regions, that transmission from the region of increased activity will occur. In the absence of highly specialized conducting paths, we find that protoplasmic excitation apparently undergoes a decrement in intensity or effectiveness with increasing distance from the point of origin, so that an excitation gradient results. Many facts, some of which are considered in the following chapter, indicate that the physiological relation resulting from the differential exposure of a cell or cell mass is primarily a relation of excitation and transmission and that the resulting gradient is essentially the fixation or establishment of an excitation-transmission gradient in the protoplasm through the modification of the protoplasmic subtratum by the persistence or repetition of the differential exposure. From this viewpoint the gradient represents the most primitive sort of excitationtransmission relation and its effects upon protoplasm.

\section{CONCLUSION}

It is perhaps necessary to point out that the conception of axiate pattern as primarily a quantitative gradation in physiological condition is nothing more than an attempt to interpret certain aspects of the physiology of development. The axial gradients do not create anything, they are not the "cause" of growth or differentiation in the organism, they do not determine what organs shall develop in a particular protoplasm. Granting their existence, and granting the significance which I have assigned to them, the gradients represent merely certain physiological conditions, under which the hereditary mechanism of a protoplasm gives rise 
to the order or pattern which we call axiate or axiatesymmetrical. ${ }^{\mathrm{x}}$

The egg in most cases requires fertilization or the action of some other factor external to it to initiate development, but the specific hereditary constitution of the egg protoplasm with its potentialities of development is present, whether fertilization or initiation of development by other means occurs or not. Physiologically speaking, the spermatozoön, or some other factor, merely sets the mechanism in motion or gives it the necessary speed, and development proceeds. Similarly, the gradient in the egg, whether it persists from earlier cell generations, or arises anew in the egg through differential exposure, is merely a physiological condition which determines that the hereditary mechanism shall give rise to a particular order or pattern. Alteration of the gradient relations alters the pattern, though the hereditary mechanism remains the same. The gradient is then nothing more than one of the physiological conditions under which the development of axiate organisms occurs and the surface-interior relation, whether a gradient or not, is merely another even more general physiological condition of organismic development.

The idea of a quantitative gradient in physiological condition as the condition initiating axiate development

I In his recently published Vertebrate Zoölogy (I920), Newman has employed the gradient conception in connection with various problems of vertebrate evolution. I have found it desirable thus far to consider the gradient primarily as a physiological factor in individual development rather than as itself a factor in evolution, but even if the direct transfer of the conception to the evolutionary field is justifiable, some of the applications which this author has made seem to me to be at least open to question. In the present state of our knowledge, however, differences of opinion concerning the scope and limitations of this, as of most other biological conceptions, are to be expected. 
and of its origin in the final analysis through the action of an external factor does not require or depend upon any particular assumptions or theories concerning inheritance or evolution. Some of the critics of the conception have regarded it as Lamarckian, but it is not, though of course it might be used readily, if desired, in a Lamarckian way. Strictly speaking, it has nothing directly to do with either inheritance or evolution, except in so far as it maintains that axiation or polarity, and symmetry are not inherent properties or characteristics of protoplasm. It is fundamentally a physiological conception formulated on the basis of many different lines of observational and experimental evidence, and while its formulation in the present state of our knowledge is necessarily incomplete and perhaps vague, I believe a fair consideration of the evidence now available justifies the conclusions advanced. 


\section{CHAPTER IV}

EXCITATION AND TRANSMISSION IN RELATION TO AXIATE PATTERN

\section{THE EXCITATION GRADIENT}

The determination of a region of greater activity in a cell or cell mass by differential exposure is merely the first step in the establishment of a physiological gradient. Living protoplasm is irritable or excitable, and excitation in a broad sense is conceived to consist in an acceleration of the fundamental activities of protoplasm or, more particularly, those concerned with energy liberation. A region, the activity of which has been increased by the action of some external factor, whether such factor be oxygen or a stimulus in the stricter sense, is a region of excitation as compared with other regions. Protoplasm also possesses the capacity to transmit excitation to a greater or less degree or, more strictly speaking, to transmit some sort of dynamic change resulting from excitation at one point, which induces excitation in adjoining regions. These may in turn excite still other regions, and so the process of excitation spreads, irradiates, or is transmitted. In the absence of highly specialized conducting paths in protoplasm we find, however, that this capacity for transmission is apparently limited by distance. In other words, the process of excitation undergoes a decrement in intensity or effectiveness with increasing distance from the point of origin and at a greater or less distance 
disappears or becomes ineffective. There is a large body of evidence for the existence of such a decrement in plant tissues and in the simpler animals. ${ }^{\mathbf{I}}$ It is a familiar fact of observation in the simpler organisms that the effects of slight stimuli are effective only over short distances, while those of more intense stimuli are effective over greater distances, perhaps throughout the whole organism. If the energy concerned in the excitation process is derived from the original excitation, and transmission is merely the transmission of this energy without any further energy liberation at the various points of the transmission path, a decrement is readily accounted for as due to the work done in the course of transmission. If, on the other hand, energy is liberated at each point affected by the transmitted change, a decrement and limited range of effectiveness is possible only in protoplasm in which a relation between the energy or the intensity of stimulus and the energy or the intensity of excitation exists, in other words only when the same protoplasm is capable of different degrees of excitation and when the energy or the intensity of stimulus decreases in the course of transmission. In the transmission of excitation in the less highly specialized protoplasms such a relation evidently does exist. It is only in tissues for which the "all-or-none" law holds good, that is, in which maximal excitation occurs with any stimulus above the threshold, that transmission of excitation without decrement is

I As regards plants see Bose (1902, I906, I907, I913), Fitting (I907 and references given), Kretzschmar (I904), Pringsheim (I9I 2). For a general discussion of transmission in animals, see Verworn (1913), particularly chap. vi and references there given. 
possible, but it may be pointed out that even in such tissue a transmission decrement may occur, if the transmission path is of such a kind that the maximal excitation decreases along it. Certain kinds of muscle tissue and medullated nerve have been found to react according to the "all-or-none" law, although even the medullated nerve, the most highly specialized of conducting tissues, shows a decrement when cooled, partially asphyxiated or partially anesthetized. ${ }^{\mathrm{I}}$ But we are concerned rather with general protoplasmic excitation and transmission than with the highly specialized conductive processes, and concerning the existence, at least very generally, of a decrement in such transmission there seems to be no doubt.

When a region of relatively high activity or of excitation is established by the action of an external factor in a protoplasm which is not specialized with reference to transmission, transmission from this region must occur to some extent, and from our knowledge of protoplasmic transmission in general we must expect that a decrement in intensity or in effectiveness of the transmitted change will occur. In other words, excitation and transmission in protoplasm in general usually give rise to an excitation gradient. The evidence at hand concerning the existence and origin of the axial gradients

${ }^{\mathrm{I}} \mathrm{As}$ regards nervous conduction see Biedermann (I903 and references), Boruttau (I90I), Ducceschi (Igor), Fischer (IgII), Lodholz (I9I3), Lucas (I9I7, particularly chaps. ii-v and references). The question of the normal occurrence of a decrement in certain regions of the nervous system is also considered by Lucas. Tashiro has observed a gradient in $\mathrm{CO}_{2}$ production in certain nerves, the rate decreasing in the direction of functional conduction (Tashiro, I9I4, I9I $5 a, b$, I9I 7 , particularly chap. iv). I have found that susceptibility in certain nerves decreases in the direction of functional conduction (Child, IgI $4 a$ ). 
in organisms, which was briefly discussed in the preceding chapters, indicates that these gradients originate as such excitation gradients and become more or less permanently fixed or established in the protoplasm through the persistence for a certain length of time of the differential exposure, the region of activity or excitation determined by it and the resulting excitation gradient.

\section{THEORIES OF EXCITATION AND TRANSMISSION}

As a basis for further consideration of the problem some discussion of excitation and transmission in general is necessary. Various theories of excitation and transmission have been advanced in the past, but most of them are either highly speculative or too exclusively concerned with the process as it occurs in nerve. ${ }^{\mathrm{I}}$ These theories differ both as regards the cause of the electromotor phenomena characteristic of excitation and transmission and their rôle in the process of transmission. According to some authorities the electrical changes are merely an incidental feature of the process, a result or a by-product, so to speak, while others hold that they are the chief factors in transmission. Again, according to some, the electrical changes have a purely physical basis, while others believe that chemical reactions are concerned in the excitation process and in bringing about the electrical changes. Some twenty years ago Waller (I897, I903) maintained that excitation is associated with metabolic activity and more recently Tashiro (I9I7 and earlier papers) has shown

${ }^{I}$ For discussion of various theories of excitation and transmission see Biedermann (I896, I903), Bose (I902, I906, I907, I913), Morat (I906), Verworn (I9I3), Bayliss (I9I8, chaps. xiii and xxii and references), also the various textbooks of physiology. 
that excitation, even in the nerve fiber, is accompanied by increase in $\mathrm{CO}_{2}$ production and holds that irritability and excitation in general are associated with oxidative metabolism.

Bayliss and Lucas have criticized these conclusions on the ground that Hill (I9I2) has been unable to discover any appreciable heat production associated with the transmission of the nervous impulse, and have pointed out the possibility that the $\mathrm{CO}_{2}$ produced by nerves may be dissolved in the tissues, and that its increase on electrical stimulation may be due to the rise in temperature occasioned by such stimulation. The demonstration by Tashiro and by Riggs (1919) that chemical stimulation, as well as electrical stimulation, is accompanied by increase in $\mathrm{CO}_{2}$ appears to angwer this objection. But whatever the situation may prove to be in the highly specialized excitatory process in the nerves of higher animals, there seems to be no doubt that protoplasmic excitation in general involves metabolic and particularly oxidative reactions. Excitation in its most primitive form apparently involves in one way or another an acceleration in the fundamental activities of living protoplasm, particularly those concerned in the liberation of energy.

In a series of papers R. S. Lillie ${ }^{\mathrm{r}}$ has developed a general theory of excitation and transmission based on extensive experimental evidence and in terms of current physicochemical conceptions. Since this theory is concerned to some extent with the process of excitation and transmission in general and not merely with the process

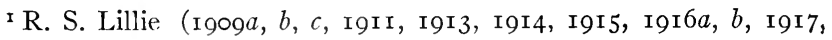
I9I8, I9I9), R. S. Lillie and E. N. Johnston (I9I9). 
as it occurs in the highly specialized tissues, it will serve as a point of departure for interpretation of the facts in the following discussion. It may be pointed out, however, that the conception of the gradients and their significance in the organism do not depend upon this or any other particular theory of excitation but upon the observed facts.

The starting-point of Lillie's theory is the generally accepted fact of electrical polarization of the plasma membrane, the existence of which is well established by the work of numerous investigators. This polarization is usually regarded as determined by the fact that the membrane in the unexcited condition is normally permeable to ions of one sign, and impermeable, or less permeable, to ions of opposite sign. The facts indicate that the unexcited plasma membrane acts as if permeable or reversible to certain cations and impermeable or less permeable to certain anions which are present in higher concentration in the cell than in the surrounding medium. The evidence indicates further

that the change in permeability associated with stimulation is not a direct effect due to merely physical changes in the protoplasmic surface layer, but is the consequence of a chemical reaction which alters the character of the surface film and temporarily deprives it of its normal semipermeable and electromotor properties, and that this chemical process may in a highly irritable tissue like nerve be initiated by any slight local decrease in polarization, provided the change is sufficiently rapid [R. S. Lillie, I9I4, p. 443].

In various other papers (e.g., R. S. Lillie, I9I7, pp. 8082 ; I9I8, p. 3 ; I9I9, p. 458) Lillie points out that the chemical change involved in the process is a metabolic reaction, probably oxidative in nature. Whether the 
primary change in the membrane consequent upon depolarization is this chemical reaction or a change in colloid condition, or some other change is at present uncertain, but since the whole series is indissociable and more or less mutually related it makes little difference which we call primary and which secondary. For present purposes the significant fact is that, according to Lillie, excitation involves a chemical change, probably oxidative in nature, as well as other changes, in the surface layer of protoplasm.

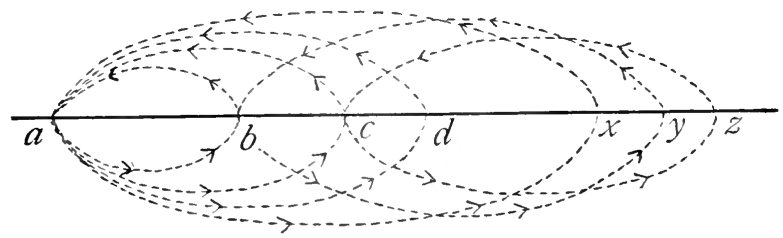

FIG. 6.-Diagram indicating the electric currents arising in relation to an excitation gradient; the direction of migration of the positive ions is indicated; for further explanation see text.

The process of transmission of these changes from point to point is accomplished by the electrical changes which constitute one feature of the excitation. The excited region (Fig. $6 a$ ) becomes electronegative (externally) to an unexcited region ( $b, c$, etc.), in other words a local electric circuit is established, the positive current passing in the cell or tissue from the region of excitation to unexcited regions with a "return" current in the external medium. In consequence of resistance the strength of this current decreases with increasing distance from the region of excitation $a$, that is to say, a gradient in electrical potential must arise in relation to a region of excitation. Lillie points out that this local 
electric current arising in relation to the excitation of a given region $a$ affords the condition necessary to bring about excitation at $b, c$, etc., i.e., at all points within a certain distance from $a$ where the strength of the current is sufficient to bring about the changes determining excitation. In short, according to this theory of excitation and transmission, the electrical changes involved in the excitation of any point $a$, however that excitation be produced, determine the transmission of excitation from $a$ to $b, c$, etc. The important point for present purposes is that in consequence of the increasing resistance with increasing distance from the point $a$, a gradient in electrical condition of the plasma membrane results from the excitation at $a$, the (external) electronegativity decreasing from $b$ to some point at which the resistance becomes so great that the electrical effect of the excitation at $a$ is zero. If Lillie's conception is correct every local excitation gives rise for the time being to such a gradient.

According to Lillie, then, the electrical change produced by excitation at $a$ becomes the "stimulus" which brings about excitation at $b, c$, $d$, etc., at all points, that is, within the limit of its physiological effectiveness. If it is true, as the facts indicate (see pp. 80-8I), that in protoplasm in general, where specialized conducting paths do not exist, a relation between the energy or the intensity of excitation and the energy or the intensity of stimulus exists, it follows that when excitation occurs at a given region $a$ of such protoplasm a gradient in the degree, energy, or intensity of excitation, i.e., in its ability to act as stimulus for other points, must result in consequence of the electrical conditions, 
this ability decreasing from $a$ through $b, c, d$, etc., to a point $x$ where the electrical change becomes so weak that it is incapable of acting as a stimulus. Moreover, the current resulting from excitation at $a$ tends at the same time to re-establish the polarization at $a$ and so to reverse the depolarization and restore the original condition of polarization at $a$.

Each new point $b, c, d$, etc., excited by the current arising from $a$, becomes the source of a current which, as Lillie has shown, may produce excitation at further points and might also produce re-excitation at $a$ if the refractory period following excitation did not prevent. It is evident that according to this conception the velocity of transmission depends upon the velocity of the local processes in the membrane at each excited point and is entirely independent of the velocity of electrical transmission which is practically instantaneous. Moreover, in any case in which excitation varies directly (not necessarily proportionally) with stimulus, a decrement must occur in the course of transmission. Only in certain highly specialized protoplasms for which the "all-or-none law" holds, i.e., in which any stimulus above the threshold produces maximal excitation, is transmission without a decrement possible.

\section{PRIMITIVE EXCITATION PROCESSES}

The question might be raised whether the assumptions concerning the semi-permeability and electric polarization of the plasma membrane and its depolarization in excitation are the only possible basis for the electrical phenomena associated with excitation. It has been pointed out by Lillie and others that acid 
production and consequent increase in hydrogen ion concentration at points of excitation will not account for the electrical changes, since the currents produced in this way are opposed in direction to those which are actually observed. It is very probable, as Lillie suggests, that the membrane changes may be the primary factors in highly irritable tissues like nerve, where the processes of excitation and transmission and recovery are extremely rapid, but it is possible that in excitation in general the changes in the membrane may be incidental rather than primary. In an oxidation-reduction cell the electric currents produced show the same relation to the oxidation end of the cell as do the bioelectric currents to the region of excitation. That is, the region where oxidation is going on in the oxidation-reduction cell is externally electronegative to the region where reduction is occurring. I have repeatedly called attention to the apparent impossibility of dissociating the process of excitation in protoplasm in general from an acceleration of the fundamental dynamic activities of protoplasm, particularly those concerned with the liberation of energy. If current conceptions are correct, the chief source of energy in living protoplasm is oxidation. IMoreover, it is apparently true that a region of rapid oxidation in protoplasm gives rise to electrical conditions similar to those which we observe in relation to the more highly specialized forms of excitation. While it is by no means necessary, nor at present justifiable, to maintain that oxidation is the primary factor in excitation, it apparently is true, so far as electrical phenomena are concerned, that a region of more rapid oxidation in protoplasm behaves with respect to 
regions of less rapid oxidation like a region of excitation with respect to less excited or unexcited regions, i.e., it becomes electronegative and a current similar to the excitation current is set up between it and other less rapidly oxidizing regions. If this current constitutes the exciting factor for adjoining regions and so the primary factor in transmission, then a region of rapid oxidation, however determined, may initiate a process of transmission which induces excitation, involving increase in the rate of oxidation at points within the range of effectiveness of the current, and so may give rise to an oxidation gradient. Doubtless even in such a case membrane changes are involved, but my point is merely that they need not necessarily be regarded as the most significant changes in the complex process. In some of his later papers Lillie calls attention to the probable significance of oxidation-reduction in the more primitive excitation-transmission relations.

In such a process as this the velocity of transmission may be very low as compared with nerve, so low indeed that it might in many cases scarcely be termed transmission in the ordinary sense. Moreover, in such a process a decrement is to be expected, since a relation unquestionably exists between the electrical condition and the rate of oxidation, and this being the case a gradient must result. In fact, in the extreme case such a gradient may arise without any wavelike transmission of excitation, such as occurs in the nerve, electrical transmission only being involved and determining a gradation in rate of oxidation and associated conditions from the original region of high rate to a certain limit of distance. 
Moreover, if the original region of high rate persists the electrical conditions and so the gradient as a whole must persist and become static instead of advancing in the protoplasm. The length of such a gradient will depend, first, upon the potential arising at the original region of rapid oxidation and, second, upon the conductivity of the protoplasm. In that they represent a gradation from a region of high to one of low physiological activity, such gradients are comparable to the ascending phase or front of an excitation wave in a nerve fiber, viz., that portion of the wave from the region where excitation is just beginning to the region of maximal excitation. But they differ from the ascending phase of the nervous impulse in that they do not advance, though they may undergo change in length with change in the rate of reactions in the original region or at other levels.

It seems probable that the most primitive excitationtransmission phenomena in protoplasm possess more nearly the characteristics or such oxidative or metabolic gradients than those of the highly specialized sorts of excitation and transmission processes, such as the nerve impulse. Apparently all gradations exist between the two extremes, and it is at least possible that the more rapidly arising and more rapidly reversible excitationtransmission processes, even in protoplasm in general, are chiefly or wholly plasma-membrane processes, while the slower, more persistent, and less readily reversible processes approach more closely the oxidation gradients and are not necessarily limited to cell membranes, but may occur in relation to limiting surfaces or phase boundaries more or less distant from the surface. But since protoplasm is a system in which a considerable 
degree of correlation and mutual dependence exists among the essential conditions and reactions, change in any essential factor cannot proceed very far without involving changes in other factors. Undoubtedly, therefore, oxidation is not independent of the condition of membranes or phase boundaries and, on the other hand, the condition of these is not independent of oxidation. In other words the most primitive and the most highly specialized forms of excitation and transmission in protoplasms probably involve much the same complex of factors, though the specialization of different protoplasmic mechanisms may determine various modifications of the general process with different factors predominant in different mechanisms. It is possible, for example, that in nervous excitation and conduction oxidation may play a rôle of minor importance, and it is even conceivable that in certain protoplasmic mechanisms some other reaction than oxidation may be involved. Again, in some mechanisms the plasma membrane and its changes may be of fundamental importance, while in others this factor may perhaps be secondary and incidental to others. Unquestionably the protoplasmic system affords a basis for the specialization of various mechanisms of excitation and transmission, but it does not follow that the primitive process is exactly like any one of the more highly specialized processes. The general problem has apparently been somewhat obscured by the fact that a large part of the work on excitation and transmission has been concerned with the process as it occurs in the muscle and nerve of the higher animals, and this work has constituted the chief basis for generalization. It is commonly held, for example, that 
excitation is transmitted as a wavelike change and that it is completely reversible. This is undoubtedly true in large measure for certain protoplasmic mechanisms, but it does not follow that it is true for all or under all conditions. In fact the existence and persistence of the metabolic and physiological gradients in protoplasms without specialized mechanisms indicates, if it does not prove, that neither wavelike transmission of the excitation process nor complete reversibility is a universal characteristic of the excitation-transmission relation. As I have pointed out, the excitation itself does not necessarily travel in these gradients, but the electromotor changes determine different degrees of excitation at different distances from the point of original excitation. Moreover, in most protoplasms these gradients are not rapidly reversible but persist after the factors inducing them have ceased to act, and become factors in determining the developmental order and relation of parts. They are in a sense fixed or static excitation gradients, and the electrical changes rather than the excitation itself are transmitted. The various lines of evidence which have been considered in preceding chapters seem to me to indicate that the primitive excitationtransmission relation in protoplasm approaches more or less closely this condition and that oxidation is an important factor in it. With the specialization of protoplasm and the appearance of one mechanism or another changes occur. Perhaps the most significant of these changes is the change in the relation between the exciting factor and the excitation produced. In the more primitive mechanisms, as pointed out above, the energy, the intensity, or, in lack of definite knowledge 
we may say, the degree of excitation, is certainly under some if not all conditions a function of the energy, the intensity, or the effectiveness of the exciting factor, and the same protoplasm is capable of different degrees of excitation. But with the specialization of mechanisms of excitation and transmission, the relation apparently becomes more and more like that of the spark and the powder, and such specialization reaches its final terms in the more highly differentiated muscle and nerve which apparently react according to the "all-or-none" law, i.e., maximal excitation results from any stimulus above the threshold. Under these conditions transmission of the excitation as a wave to an indefinite distance without decrement is possible, provided the maximal excitation at successive points of the path does not decrease, in other words, provided the path is not already a physiological gradient.

In conditions intermediate between the two extremes transmission may occur to a greater or less distance beyond the limit of the gradient resulting from the original excitation, but with a decrement. If, for example, the excitation induced at a point $b$ (Fig. 6) by the electrical changes resulting from excitation at $a$ gives rise to sufficient electric current to increase the strength of the current produced at $a$ the limit of effectiveness will be not $x$ but a more distant point $y$. In this case then the excitation at $b$ will become a factor in determining excitation at a point beyond the range of the current produced at $a$ and the length of the excitationtransmission gradient will be increased. Similarly, excitation at $c$ may be sufficient to determine excitation at $z$ and so on. In such a case transmission is not limited by 
the conditions arising at the point $a$ of original excitation, but points $b, c$, etc., become factors in extending the range. Unless the degree of excitation at the various points $b, c, d$, etc., within the range of the current produced at $a$ is the same and therefore independent of the strength of the current at these points, the excitation will undergo a decrement in the course of transmission and will finally reach a limit, and the steepness of the decrement will differ according to the relation between stimulus and excitation in the protoplasm concerned. The facts indicate that between the primitive gradient in which transmission depends entirely upon conditions at the point of original excitation and the condition of transmission without decrement various intermediate conditions exist, even in the same organism at different stages of development and perhaps in some cases with different degrees of excitation (see p. 225).

\section{THE QUESTION OF REVERSIBILITY}

When we take into account all the facts it seems necessary to conclude, as I have already suggested, that excitation in a broad sense is essentially nothing else than an acceleration of the fundamental activities of life, and particularly or primarily those concerned with energy liberation. Excitability or irritability is a fundamental characteristic of living protoplasm and life is apparently made up of a series of excitations and their effects. The processes may differ as the mechanisms differ, but the fact remains that excitation means a rise in the level of living. The physiological or metabolic gradients which, as I have endeavored to show, constitute the fundamental features of axiate pattern, are essentially gradients 
in the rate of living, possess all the characteristics of excitation gradients, and are determined by differential exposure to the action of external factors. I believe we are amply justified in the light of all the facts in regarding them as the most general and primitive expression of the excitation-transmission relation in protoplasm.

The fact that these gradients are usually more or less persistent and not readily or rapidly reversible requires some consideration. From our knowledge of momentary excitations and the highly specialized processes in nerve and muscle we are accustomed to think of excitation as completely reversible. Excitation may be completely reversible in the fully developed nerve fiber, but certainly in many parts of the nervous system it is not completely reversible, as memory and the possibility of learning show clearly enough. Moreover, it is evidently not completely reversible in muscle, since frequent excitation, if not exhausting, leads to growth, i.e., functional hypertrophy, and after a certain stage of development excitation is necessary for the continued existence of the muscle. Whether we invoke a mysterious "over-compensation" or some other hypothetical process to account for these facts, they show that while muscular contraction is completely reversible the process of excitation as a whole is not.

The fully developed organism represents an approach to dynamic equilibrium. The protoplasmic record of the events of development is about as complete as is possible for the protoplasm concerned. The various mechanisms are all developed and the further changes in them are largely temporary and reversible except so far as the gradual changes which constitute senescence 
are concerned. That is to say, the fully deveioped organism represents a condition in which most of the irreversible or less readily reversible changes possible in a particular protoplasm under the conditions of life and development have already occurred. It is, in fact, the occurrence of these changes which constitutes development. Under altered conditions reversal, or more properly as regards the process as a whole, regression may occur, at least in the simpler organisms with less stable structural features, and the protoplasm may return to or approach an embryonic or unspecialized condition and undergo development anew (Child, I9 $\left.5^{b}\right)$. The earlier stages of development, however, do not and cannot be expected to show the high degree of reversibility of processes that we find in the fully developed organism. Embryonic protoplasm is protoplasm from which the developmental record has been, so to speak, wholly or in large measure erased, and development is the recording process.

I have tried to show that the physiological gradient is the first step in the developmental record. If oxidation and synthesis are associated, as many authorities now believe, the higher rate of oxidation at the higher levels of the gradient must be a factor in determining a higher rate of synthesis, and more rapid growth may occur at these levels than elsewhere, as is often the case. Moreover, different molecules may be synthesized, or different products of synthesis may be relatively stable and remain in the protoplasm, at the different levels and so differentiation may begin. The chief point for present purposes is that if the conditions giving rise to the gradient persist long enough, whether this be hours, 
days, or weeks, the gradient usually becomes to some extent more or less permanently recorded in the protoplasmic substratum. Concerning the exact nature of this record we can only say that it consists primarily in the factors determining the rate of fundamental protoplasmic activity, for we see that the differences in rate persist. The persistence of the gradient as a gradient must depend upon the fact that the changes in the protoplasmic substratum go on more rapidly at the higher levels than elsewhere. In short, I believe that the determination by an external factor of a more or less persistent gradient in physiological condition is the most general case of incomplete reversibility of excitation and also of so-called functional adaptation, that is, the process of the more or less persistent alteration of protoplasmic structure and function by the dynamic activity occurring in it.

\section{GENERAL DISCUSSION}

Concerning the existence of the axial gradients and their significance in development there can be no doubt. These are demonstrated facts, however we may interpret them, and they are the earliest indications of an order or pattern on a larger scale than that of protoplasm. Organismic pattern is, as already noted, a pattern of regions or masses of protoplasm or cells and of a higher order of magnitude than protoplasmic pattern; physiological polarity and symmetry appear in protoplasms of very different specific constitution and many different lines of evidence agree in indicating that they are primarily quantitative gradations in physiological condition, and that they can be 
determined experimentally in many protoplasms by differential exposure of the cell or cell mass to the action of an external factor. In some protoplasms the gradients are only transient. In Amoeba, for example, the ectoplasm of the pseudopod is a gradient for the time being, but here the changes which give rise to the gradient are readily reversible and no permanent axiation develops (Hyman, I9I 7). In most protoplasms, however, the changes are less readily reversible, and a definite axiation results which may persist even through reproductive processes. The development of the axial gradient is merely one feature of development in axiate forms, and it represents the first step in the localization of physiological differences of organismic magnitude in the protoplasm concerned. The course of further development and differentiation is determined by the specific hereditary mechanism of each particular protoplasm and the gradient is merely the framework, the ground plan on which the complex superstructure arises.

We have seen (p. 33) that in the simpler forms and earlier developmental stages the axial gradient is very commonly found only in the superficial regions of the body. In most Protozoa and differentiated plant cells this superficial region is apparently denser and more permanent in position and structure than other regions of the cytoplasm, and if the gradient is an excitation gradient it may be expected to appear primarily or chiefly in relation to limiting surfaces, and its persistence is possible only in those regions in which the protoplasmic substratum possesses a certain degree of stability. But, admitting that excitation is transmitted along limiting surfaces, such surfaces are present, not 
only at the cell surface, but may be more or less continuous in the interior according to the phase relations. There is no reason why excitation should not be transmitted along these internal limiting surfaces, so far as they are continuous, and in this way penetrate the protoplasm to a greater or less depth. The internal limiting surfaces are doubtless not identical with the external, and conditions in them may be less favorable, but there are no grounds for believing that internal transmission is impossible. If it is possible, the axial gradients may exist not only at the surface but to a greater or less depth in protoplasm, according to conditions, and may in some cases involve the whole cell, as is apparently the case in some eggs. This suggestion also bears upon the question of cell pattern and surfaceinterior pattern in general (p. 6o). I believe, in short, that the interpretation of axiate pattern in terms of excitation and transmission and the more or less persistent developmental protoplasmic changes which result from it is not only the simplest interpretation possible on the basis of our present knowledge but the interpretation which accounts most satisfactorily for all the known facts.

It is impossible to review the literature of the subject here, but it is of interest to note that many authors have suggested that polarity is a gradient of some sort. Morgan (1904, I906, 1907), for example, has at various times advanced the idea of polarity as a gradation or stratification of materials. Boveri (I9IO) maintained that the polarity of the Ascaris egg must be a gradation of some sort, and numerous authors have described gradations in size or form of parts, rate of development 
or regeneration, etc., along the chief body axis. In fact, evidence of one kind or another for the existence of gradients of some sort as characteristic features of axiate organisms appears in the literature of many different and widely separated fields of investigation, both physiological and morphological. This evidence is perhaps the more valuable because the authors were in most cases stating or describing facts rather than attempting to formulate theories. In the conception of the excitation gradient and its fixation in protoplasm I have merely attempted to provide a general physio. logical basis for the facts as they stand. 


\section{CHAPTER V}

\section{THE PHYSIOLOGICAL GRADIENTS IN RELATION TO ORGANISMIC INTEGRATION}

It is evident that a simple excitation-transmission relation constitutes, at least momentarily, a physiological integration, in which the point of primary excitation becomes the dominant or controlling factor, since it initiates the transmitted change and determines what shall occur at the various points along the path of transmission. Moreover, this relation is the physiological basis of nervous function and integration, and this in turn represents the highest degree of physiological integration which has been attained in the course of evolution. It was pointed out above (p. I7) that the excitationtransmission relation is the most general and most primitive relation of organismic as distinguished from purely protoplasmic character, and I have endeavored to show that the axial gradients originate as excitation gradients. From this viewpoint the excitation-transmission relation appears as the basis both of the beginnings and of the most complex and highly developed features of organismic integration, viz., nervous integration. Such a conception has at least the advantage of placing the problem of organismic pattern and integration before us in definite and consistent physiological terms.

The rôle of the physiological gradient as an integrating factor follows necessarily from the fact that it is a gradient. Once established it brings different regions 
within its range into a definite physiological relation. The differences in electric potential which have been found to exist between different levels afford one means of actual correlation, and, if Lillie's theory is correct, the resulting electric currents must be the primary factors in determining and maintaining the conditions at different levels. Conceived in these terms, organismic integration is primarily electrical, that is to say, the factor actually concerned in the first instance in bringing different protoplasmic regions into physiological relation with each other is the electric current. But this is only the first step in organismic integration. As soon as differences in protoplasmic constitution appear at different levels of a gradient, chemical or transportative correlation begins to play a part in integration, and still later various mechanisms of correlation and integration develop, e.g., the various types of vascular system, the supporting and other mechanical tissues, the organs of secretion and excretion, and in most animals the nervous system as the organ of integration par excellence.

\section{THE PHYSIOLOGICAL GRADIENT AS A BASIS FOR} DIFFERENTIATION

Granting that the physiological gradient is the basis of axiate pattern, it is evident that it must constitute the starting-point of the orderly, progressive complication in differentiation and physiological correlation which we call development. In some way qualitative differences must arise in the protoplasm at different levels of the gradient which was essentially quantitative in its origin. The origin of differentiation from differences which are primarily quantitative may appear to 
some to constitute a formidable difficulty for this conception, but I believe the difficulty is only apparent. It is of course true that differences in the velocity alone of a pure chemical reaction do not give rise to qualitatively different products of reaction. In protoplasm, however, we are concerned, not with a pure chemical reaction, but with an exceedingly complex system, involving both physical and chemical factors. The coefficients of quantitative alteration of the different factors of the protoplasmic system in relation to quantitative external factors, such, for example, as temperature, are of very different magnitudes. The temperature coefficients of most chemical reactions differ widely in magnitude from those of many physical processes, and there is even considerable difference in the temperature coefficients of different chemical reactions. A change in temperature then does not alter the different component factors of the protoplasmic system to the same degree, but alters some much more than others. Consequently such a change, which is nonspecific and quantitative for each factor, may alter the system as a whole in such a way that chemical reactions which could not take place before the change may occur after it, and vice versa. Changes in concentration of reacting substances do in many cases determine different products of reaction, and change in temperature may bring about changes in concentration of substances in protoplasm in various ways, e.g., by alteration in the water content, the dispersion of the colloids, adsorption, and by change in the rate of chemical reaction itself, which may change the concentration of the products of a given reaction and so determine a second reaction which could not take place 
previously, and therefore a new product of reaction. Such changes determine others, and there is not the slightest doubt that a change in protoplasmic condition, originating as a purely quantitative alteration in some factor or factors, may become the starting-point of extensive qualitative changes.

When we take into account the material exchanges between protoplasm and its environment, various other possibilities of change in concentration of substances in the protoplasm appear. An increase in rate of oxidation in the protoplasm may oxidize certain substances more rapidly than they enter the protoplasm, and, as they disappear, other substances, previously inactive, may enter reaction and different products be formed. As a matter of fact, even with our present fragmentary knowledge of living protoplasm, almost endless possibilities appear for the origin of qualitative differences from primarily quantitative changes in the system. It may even be questioned whether it is possible to bring about a change in protoplasm which remains throughout purely quantitative.

Differentiation in the organism consists, at least very largely, in the appearance in protoplasm of substances not present originally in appreciable quantities, and such substances differ in amount or in kind in different regions or cells. In many such cases a simple difference in relation between the rate of nutritive intake and the rate of oxidation is sufficient to account for the appearance of a particular substance in a certain region or at a certain time, or its non-appearance or disappearance in another region or at another time. The accumulation and disappearance of fat and various other so-called 
reserves, the loading and discharge of gland cells, are cases in point, and there can be no doubt that many other differentiations arise in essentially the same way. That is, a region in which oxidation is occurring slowly becomes different from a region of rapid oxidation because of this difference in relation between intake and rate of oxidation, which determines that in the one region certain substances shall accumulate as part of the structure, while in the other such substances are more or less completely oxidized as rapidly as they are formed.

The changes concerned in excitation, whether they are primarily electrical, chemical, or physical, may similarly become the starting-point of a series of qualitative changes, particularly if they persist for a considerable time, and it is evident that the different levels of an excitation gradient or a more or less permanent physiological gradient afford numerous possibilities for the origin of qualitative differences at the various levels. Such changes may or may not be readily or completely reversible. The changes which constitute differentiation are those which are not readily or completely reversible under the conditions of development.

As soon as these qualitative differences make their appearance the basis for definite and orderly chemical or transportative correlation of organismic magnitude is established, and from this time on such correlation plays an increasingly important rôle in the further sequence of events. The definiteness, variety, and specificity of chemical correlation must increase as differentiation progresses and mechanisms of communication develop, both in the individual and in the course of evolution, 
until in the higher animals and man it becomes almost inconceivably complex. Moreover, from the moment of its appearance as a factor in organismic pattern, chemical correlation constitutes a factor in organismic integration, which develops along different lines from nervous integration and makes possible other kinds of control and relation than those determined through the nervous system. The internal secretions and all so-called hormones of the higher animals and man constitute the most advanced stages attained in this development.

Differentiation appears, not only in the development of the mature organism from the egg, but in many animal eggs the appearance of the yolk in the protoplasm constitutes a characteristic differentiation. The relation of this differentiation to the physiological gradient in the egg is of interest. Very commonly a gradient in yolk accumulation appears, the apical ("animal") pole of the egg showing little or no yolk and the amount increasing toward the basal ("vegetative") pole. Yolk is substance which can under certain conditions be oxidized and furnish energy, but it is evidently not oxidized to any great extent during the growth period of the egg in the region where it accumulates, and it has been suggested that a reduction is involved in yolk formation. Evidently the different regions of the egg are different in some way, and the gradient in yolk accumulation suggests that the difference is essentially quantitative, not qualitative. Moreover, we find in such an egg a quantitative gradient in physiological activity, and when development begins the region containing least yolk shows the greatest developmental and apparently 
the greatest oxidative activity, and is certainly doing the most work. Evidently the localization of the yolk in the egg is determined by the axial gradient and constitutes the first differentiation in relation to that gradient. The facts indicate very clearly that the yolk accumulates in the region of least oxidation, even though the substances from which it is formed enter at the opposite pole, as they do in many cases (Fig. 5). Such differentiation along the axis of the egg becomes the basis of the two primary germ layers, the ectoderm arising from the more active, the entoderm from the less active, region. The region of origin of mesoderm differs in different forms, but is often intermediate between ectoderm and entoderm. The gradient hypothesis maintains that differences in behavior of the different egg regions and of the germ layers arising from them result from the fact that they represent different levels of the gradient.

The gradient which determines the differentiation of the oögonial cytoplasm into yolk-bearing and yolkless regions is merely the beginning of a more or less complex series of events, each of which is determined in a definite and orderly way by the preceding. It will be evident at once that as soon as any such differentiation along a gradient occurs chemical or transportative correlation becomes possible, and it is certain that such correlation plays an increasingly important and complex rôle as development progresses. The accumulation of yolk in one region of the egg becomes itself a basis for transportative correlation, for most of this yolk is finally used, not in the region where it accumulated, but in the region which originally contained little or no yolk. 
There are of course many cases in which the gradient does not determine in the egg any such gross structural differentiation as the yolk gradient, but such differences are incidental rather than fundamental, and depend upon differences in the specific constitution of the protoplasm of different eggs, differences in nutritive conditions, etc. But sooner or later the structural differences appear, always in definite and orderly relations to the gradients, and it has been shown that the localization and relative size and proportions of parts along an axis can be altered experimentally by altering the length, steepness, or direction of the gradient. ${ }^{\mathrm{I}}$ I believe that the evidence at hand warrants the statement that it has been experimentally demonstrated that such a gradient in a specific protoplasm constitutes an adequate basis for the initiation and so for the orderly progressive complication, with reference to the particular axis which the gradient represents, of the differentiation characteristic of the process of development in that particular protoplasm. Once again it may be pointed out that the gradient does not determine the character of differentiation and the kind of organs produced at different levels in different organisms. These are determined by the specific inherited constitution of the protoplasm.

Where symmetry gradients as well as polar gradients are present the situation is of course further complicated and orderly differences arise in more than one direction. Similarly, as particular organs are localized and develop, new organ gradients or axes may arise at any angle to the original axis in consequence of local conditions, until in the higher animals the axial relations

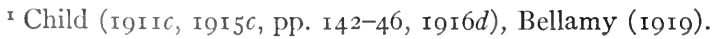


may become almost inconceivably complex. In the mammalian nervous system, for example, the polarities of the different neurons form all possible angles with the primary body axes, yet it seems evident that these polarities arise in response to local conditions.

\section{PHYSIOLOGICAL DOMINANCE AND SUBORDINATION IN RELATION TO THE AXIAL GRADIENTS}

Turning to another aspect of physiological integration, it is evident that a relation of dominance and subordination is a characteristic feature of the axial gradient. The region of most intense activity, the high end of the gradient, must influence the physiological condition at other levels to a greater degree than these other levels can influence it. It is the region of highest electronegativity and the primary factor in determining the electrical gradient, and therefore must dominate or control other levels to a greater or less extent. When a new excitation occurs at any level of an already established physiological gradient it must, if the electric current is the transmitting factor, as Lillie maintains, be transmitted to a greater distance down the gradient than up, because as it passes to higher levels its own electric current will be more and more completely compensated by the current arising at the higher levels. When an axial gradient is already present we may expect to find, at least in the simpler organisms, a difference of this kind in transmission with respect to the two directions, upward and downward. Figure 7 is an attempt to show in a purely diagrammatic way the reason for the disappearance within a comparatively short distance of an excitation transmitted up a physiological gradient. 
In this figure $x y$ is the gradient in electronegativity characteristic of the physiological gradient. Let us suppose an excitation to occur at $a$ with an increase in electronegativity $a b$. It is evident that the current produced by excitation at $a$ will be compensated at $d$ by the current of the physiological gradient, but if points on the gradient above $a$ increase the strength of

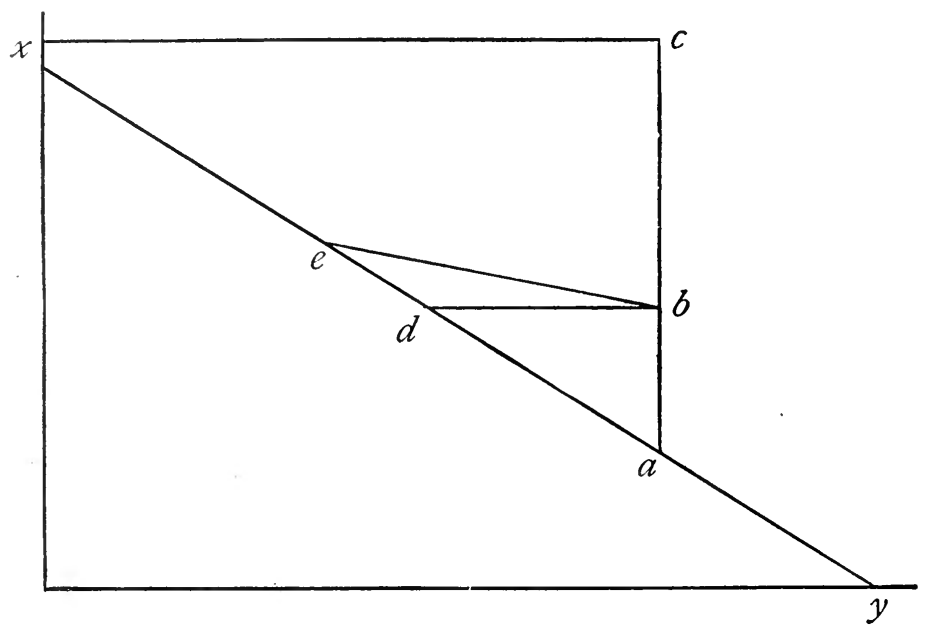

FIG. 7.-Diagram illustrating transmission up a gradient; for explanation see text.

the excitation current the excitation may be transmitted to a higher level $e$ and so on.

An excitation at the level $a$ which increases the electronegativity to $c$ will produce electrical effects at all levels up to $x$ and if the excitation brought about by this current at levels above $a$ increases this electronegativity still further, the excitation $a c$ may be transmitted all the way to the high end of the gradient and 
may produce an increase in electronegativity even there. These relations may of course be altered by various factors: for example, higher levels of the physiological gradient may and probably often do have a lower threshold or a higher maximum of excitation than lower levels, consequently the decrement may be different at different levels. In tissues which react according to the "all-or-none" law transmission may occur to an indefinite distance up a gradient, and if the maximal excitation is higher at higher levels the energy or intensity of the excitation may perhaps increase as it is transmitted upward.

Differences in transmission up and down an axial gradient appear in many functional relations. It has been noted by various observers that when flatworms or annelids are cut in two, at least in the more anterior body regions, the portion anterior to the cut is much less affected than the posterior portion (Norman, I900). Coelenterate tentacles show distinct physiological gradients, and conductivity down the gradient, that is, basipetally in the tentacle, is much better than in the opposite direction. Various other similar cases might be mentioned.

This relation of dominance and subordination, however, is not simply a functional relation in fully developed organisms, but is concerned in development and is quite independent of the presence of a nervous system. The existence of such a relation in plants with the growing tip or, in certain cases, some other region of high activity as the dominant region has long been known. This relation in the higher plants has been interpreted by Sachs, Loeb, and others in terms of 
transportation of formative or inhibiting substances, but even in these plants it can be blocked by a zone of low temperature about the stem between dominant and subordinate parts, which merely decreases the metabolic activity of the cooled region but does not stop transportation (Child and Bellamy, I919). Moreover, the relation of dominance and subordination exists in algae in which a transportative mechanism is not, so far as is known, developed.

A similar relation exists in animals, the apical or anterior region being primarily the dominant region in the polar axis, and it has been possible to show experimentally that this relation plays an important part in the localization, proportions, and relations of parts. ${ }^{\mathrm{x}}$ In Planaria, for example, a piece taken from any level of the body is capable of giving rise to all parts posterior to its own level, even if it does not give rise to a head, but it never produces any part anterior to its own level unless some degree of head development occurs first (Figs. 8-12). Moreover, the distance from the head at which certain organs arise in regeneration can be altered experimentally by altering the rate of physiological activity in the regenerating head region (Child, I9IIc). In short, any given level of the body dominates more posterior levels to a certain degree, and is dominated by more anterior levels.

Although the relation of physiological dominance and subordination originates in the physiological gradient,

${ }^{I}$ In 1907 I called attention to the existence of such a relation in the hydroid, Tubularia (Child, I907a, c, I91 $2 b$ ), and Rand's work on Hydra led him to recognize its existence in that form (Rand, I9II, I912). Since then further data bearing on the question of dominance and its relation to physiological condition have accumulated (Child, I9I I $c$, I9I4d, $e$, I9I5c, chap. iv, I9I6b). 


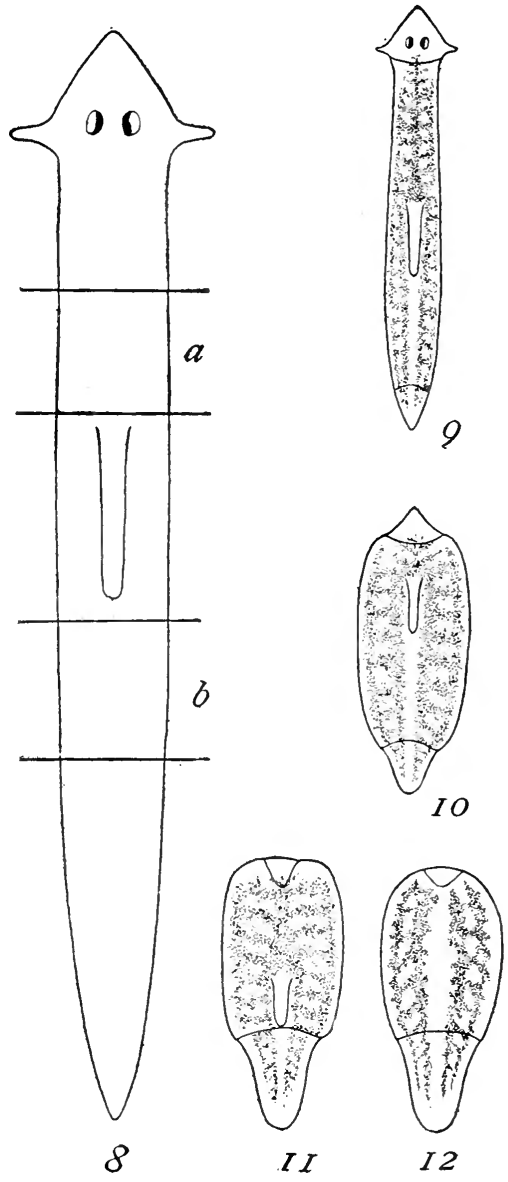

FIGS. 8-I 2.- Reorganization in pieces from different body levels, $a$ and $b$, in relation to presence or absence of a head; Planaria dorotocephala: Fig. 8, diagrammatic outline, indicating levels from which pieces are taken; Fig. 9, normal animal developing from either $a$ or $b$ when head arises; Fig. Io, anophthalmic form with rudimentary head, but developing all parts of body, whether taken from $a$ or $b$; Fig. I I, headless form from region $a$, develops all parts posterior, but none anterior to region $a$, from which it was taken; Fig. I 2 , headless form from region $b$, develops all parts posterior, but none anterior to its own level (from Child, I9I 5 c). 
it is not necessary, for the persistence of the relation, that the original gradient shall persist unchanged. After definite anatomical relations of parts and definite conduction paths have developed, the dominant region may still maintain its dominance, even though the metabolism or other physiological change in it is less in amount than in the parts which it dominates. The initiation of a nervous impulse in a receptor of the head and its conduction through the nervous system to an effector certainly does not involve a very large amount of metabolic, electrical, or other physiological change, but by means of such impulses the nervous system maintains its dominance, even though the physiological change brought about in the effector by the impulses may be far greater in amount than that in the nervous system. The physiological gradient represents merely the first step in the determination and establishment of the relation of dominance and subordination.

\section{PHYSIOLOGICAL ISOLATION}

Since this relation of dominance and subordination is a feature of the physiological gradient and since the length of the gradient is limited, the range within which such dominance is effective must be limited, though variable, according to physiological condition. In terms of Lillie's hypothesis the range of dominance must be determined primarily by the range of effectiveness of the electrical gradient which is a feature of the physiological gradient. This limitation of the range of physiological dominance makes possible the occurrence of physiological isolation, i.e., of the more or less complete isolation of a part of the body of the organism 
from the controlling or dominating action of the high end of the gradient. Such physiological isolation is a familiar fact in plants and I have shown that it also occurs in animals and that it determines at least many processes of agamic reproduction. ${ }^{\mathrm{I}}$ Such physiological isolation may occur in four ways: first, by increase in the size of the body beyond the limit of the axial gradient, the region most distant from the high end of the gradient may be isolated; second, decrease in the activity of the dominant region may decrease the length of the gradient and so determine isolation of the most distant regions without actual increase in size of the body; third, transmission between the dominant region and another part may be blocked by one means or another and parts beyond the block may thus be isolated; fourth, a subordinate part may be directly excited to such a degree that its own activity and electric potential equal that of the dominant part and the current produced by it compensates the current produced by the dominant region, consequently the part concerned is physiologically isolated.

The result of physiological isolation of a part in the simpler organisms is the same as the result of physical isolation. In all forms in which dedifferentiation

I The literature of both botany and zoölogy contains many data on physiological isolation of parts, but they have been described under various other terms. The outgrowth in plants, in consequence of inhibition of the growing tip, of buds previously inhibited by the tip is a familiar example of physiological isolation. Similar processes occur in the hydroids and other colonial animals. The occurrence of fission and budding and various other forms of agamic reproduction in many different animals also results from physiological isolation. See Child (I907 $c$, I9Ioa, I9IId, $e$, $\operatorname{Ig}_{5} c$, chap. v, I9I $7 c, d$ ), Child and Bellamy (I9I9), Bellamy (I9I9), Hyman (I9I6). 
occurs readily the physiologically isolated part loses to a greater or less extent its differentiation as a part and returns to or approaches an embryonic condition, and if it retains the original axial gradients, or new gradients arise in it in consequence of local conditions, it develops, like any other embryonic protoplasm of the species, into a new individual. In this way the occurrence of budding, fission, and the spatial arrangements of different axes in multiaxial plants and colonial animals, such as the hydroids, are determined (Child, I9I9b). Moreover, the evidence indicates that repetition of parts in series, e.g., segmentation (Child, r9r7d; chap. viii, below), and at least many other repetitive developmental phenomena are essentially similar in character. In short, any active region, whether it be the apical end of a whole organism or a special organ, such as a hydroid tentacle, dominates to some extent a certain region about it so that only outside of this region and beyond the range of this dominance is a repetition of the individual part or organ possible.

According to this conception, then, the gradients established in protoplasm may determine not only the course of differentiation along an axis but in the simpler organisms the limit of size of the individual, the occurrence and order of various agamic reproductive processes, and the orderly repetitive arrangement of parts. There is much evidence to indicate that the range of dominance increases greatly during individual development, particularly in animals in which a nervous system with a much higher conductivity than protoplasm in general develops. Physiological isolation of parts may occur in embryonic stages when the organism is very small, but 
in later stages, after the nervous system develops, the parts may be reintegrated into a new whole, as is apparently the case with annelid segments (Child, I9I7d). It is probable, however, that in the higher animals the size of the adult is determined rather by the cessation of growth with increasing differentiation than by the range of dominance, for the conductivity of the highly specialized nerve fiber is so high that the range of dominance is certainly much greater than the length of any animal body, and if medullated nerves actually do conduct without decrement, it is indefinite. But even if physiological isolation of parts of the body should occur in the more advanced developmental stages of the higher animals, it would doubtless not result in agamic reproduction of new individuals, because the capacity of the cells for dedifferentiation is limited. Certain aspects of the tumor problem, particularly with respect to the embryomas and the malignant tumor forms with indefinite growth, are of interest in this connection but cannot be considered at this time.

\section{CONCLUSION}

This brief survey of some of the more important features of physiological integration and isolation will serve to show in a general way how a quantitative physiological gradient involving differences in the rate of fundamental reactions and in protoplasmic conditions associated with them may, on the one hand, initiate localization and differentiation of parts and organs and on the other constitute the basis of a relation of dominance and subordination within the individual. Such a gradient, however, is merely the initiator and ordering 
factor in the series of events which constitute morphological and physiological development. From the first moment when qualitative differences arise at different levels of the gradients, chemical or transportation correlation becomes possible and from this time on it becomes increasingly complex and important as a factor in determining the course of events. The earlier transmissive relations between certain parts may later be supplemented or perhaps even more or less completely replaced in some cases by transportative relations. Whether or not the gradient persists as such throughout the life of the individual is a question of detail to be determined in each particular case. As a matter of fact, in the simpler organisms it does often persist, at least in the superficial regions and body wall, but it does not follow from its presence in these parts that the internal organs will show the same relations. The changes which occur between the comparatively simple physiological gradient or gradients in the egg and the situation in the adult must be determined, like any other aspect of development, by investigation in each particular case. In these changes the hereditary mechanisms of the different protoplasms are concerned. Even if the gradients in two different protoplasms were exactly similar in slope and quantitative relation the course and results of development would undoubtedly differ in the two cases. 


\section{CHAPTER VI}

LOCALIZATION AND DIFFERENTIATION OF NERVOUS STRUCTURE IN RELATION TO THE ORGANISMIC PATTERN

THE NERVOUS SYSTEM AS A PRODUCT OF PHYSIOLOGICAL FACTORS

According to the conception of organismic integration advanced in the preceding chapters such integration is, at least in axiate forms, primarily excitatory and transmissive in character, and the rôle played by chemical or transportative factors, hormones, etc., is secondary, though of great importance, particularly in the more complex, highly differentiated forms.

If the organism were primarily nothing but an aggregation of different chemical substances with the possibility of chemical or transportative relations between them, it is difficult to understand how it could ever become anything else; in other words, how a nervous system with its excitation-transmission relations could arise in it. But living protoplasm is something more than such an aggregation of chemical substances, because it is irritable, that is, it possesses the capacity for excitation and transmission and therefore, as I have pointed out, the possibilities of excitation-transmission relations between different parts exist in it. Most biologists are agreed that the appearance of a nervous system in protoplasm does not involve the origin of a new functional activity different from the fundamental activities of protoplasm in general. The current view 
is rather that nervous function develops in some way from the functions of excitation and transmission which are characteristic of all protoplasm. If this view is correct, some sort of physiological continuity must exist between excitation and transmission in general, and development and function of the nervous system.

In forms possessing it the nervous system is not only the chief organ of excitation and transmission but also the chief organ of physiological integration, and the physiological factors concerned in its origin in ontogeny must be factors connected with organismic integration in general. Chemical, transportative, or hormonic relations throw no light on the problem, and the only logical procedure is to seek these factors in the general, non-nervous excitation-transmission relations. Admitting that the physiological gradients originate in excitation-transmission relations and that they represent fundamental features of organismic pattern and integration, the developmental relations between these gradients and nervous structure and function become highly significant.

THE LOCALIZATION OF NERVOUS STRUCTURE

In the first place the localization of nervous differentiation shows a very definite relation to surfaceinterior pattern. The primary receptors develop at the surface, and the nervous system as a whole is primarily of ectodermal origin and even in the higher animals arises superficially. Even in the neutral tube of vertebrates we find indications of the influence of the surfaceinterior factor in the localization and direction of out-growth of the earlier axons. These axons arise on the 

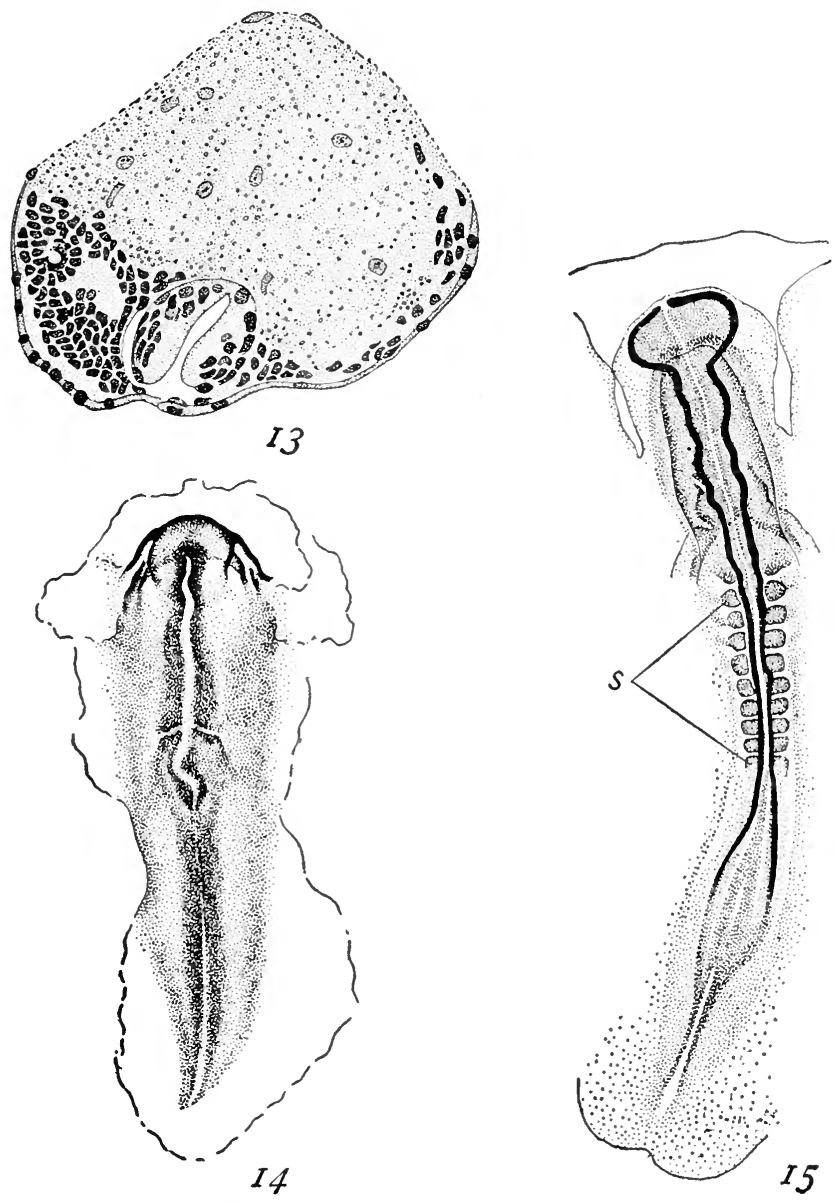

FIGS. 13-I 5. - Axial gradients in development of the central nervous system: Fig. I3, embryo of Plagiostomum girardi, a rhabdocoel turbellarian; longitudinal section near median plane with anterior end at left; the mass of cells anterior to the pharynx represents the cephalic ganglia and one eye. These organs are advanced in development, but postcephalic regions have scarcely begun to develop (from Bresslau, I904); Figs. I4, I5, surface views of two stages in development of chick, showing progress of development in posterior direction. In Fig. 14 the central nervous system is marked off as a distinct region, the medullary plate, only in the anterior head region. In Fig. I5 the neural tube has developed by closure of the medullary folds to a level posterior to the somites, $s$. Still farther posterior is the secondary region of growth (from F. R. Lillie, I9I9). 
sides of the neuroblasts which face away from the central canal, i.e., the surface originally external, and the first outgrowth of the axons is usually away from the central canal, though this direction may be altered by the axial relations (pp. 185-87). In forms in which axiation is but little developed the nervous system may be a more or less diffuse nerve not developing in relation to the body surface with little centralization, as in various coelenterates.

In animals with more highly developed axiate pattern we find the chief receptors and the chief aggregations of nervous tissue differentiating from the high ends of the chief body gradients. This relation is most conspicuous with respect to the major or polar gradient, the apical end, or the head, with its special sense organs and nervous ganglia, arising from the high end of the gradient, but it also appears more or less clearly with respect to the symmetry gradients (see p. I49); the general direction of growth and differentiation of nervous structure is down the gradients. Differentiation of nervous structure begins at the anterior end and progresses posteriorly (Figs. I3-I7) and growth of nerves is in general from central to peripheral regions, particularly in the higher animals. The direction of early axon outgrowth in the vertebrate neural tube, while showing primarily a more or less definite relation to the surface-interior pattern, as already noted, reacts very early to the axiate pattern and the axons grow in general postero-ventro-laterally, that is, down a resultant of the chief body gradients (pp. $185-87$ ).

This definitely directed growth of nerves in relation to the gradients is not limited to the higher animals. A 

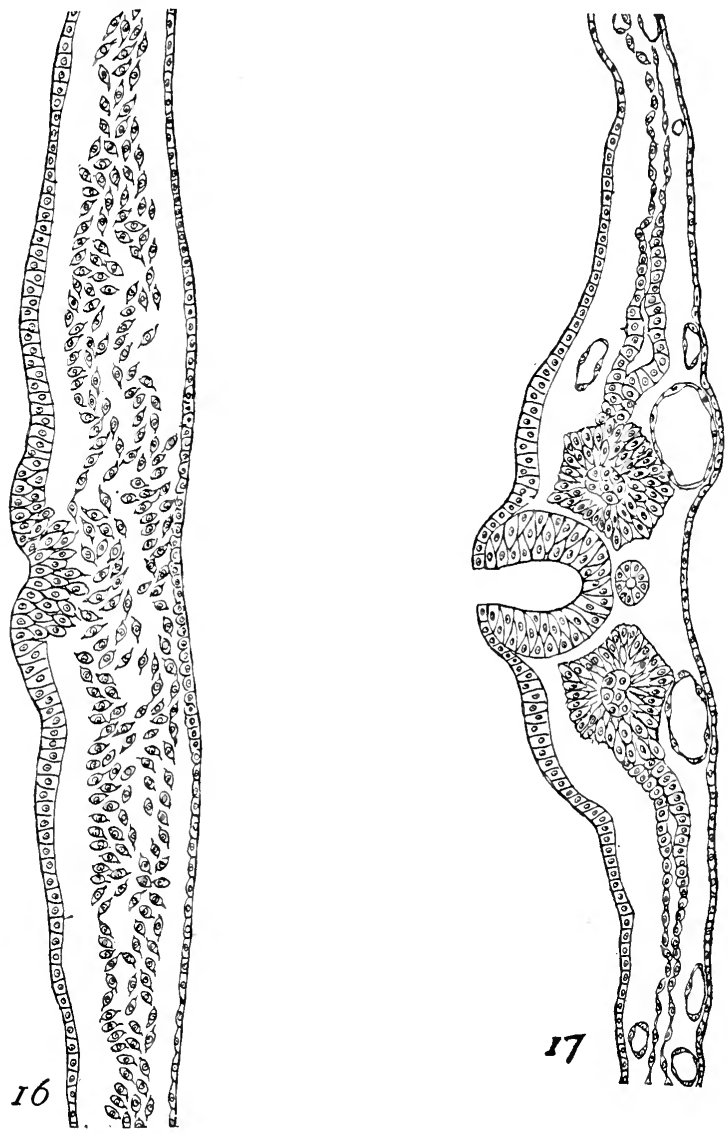

Figs. I6, I7.-Transverse sections of chick embryo at different levels, to indicate developmental gradients: Fig. I6 indicates the condition of the posterior half or more of the stage of Fig. I4 and of the extreme posterior region of the stage of Fig. I5; Fig. I 7 indicates the condition at the level of the sixth somite of Fig. ${ }_{5}$; the region of most intense developmental activity is primarily median dorsal, and development progresses in general laterally and ventrally (from Child, I9I ${ }_{5} c$ ). 
striking example of such growth of nerve fibers in an axiate organ is found in the tentacles of sea anemones. Groselj (I909) showed that the fibers arising from the sensory cells in actinian tentacles grow predominantly in the basipetal direction (Fig. I8), though in some cells the basal outgrowth divides and gives rise to fibers growing in both directions. Parker (I9I9, P. I32) concludes that the physiological polarity of such tentacles is dependent

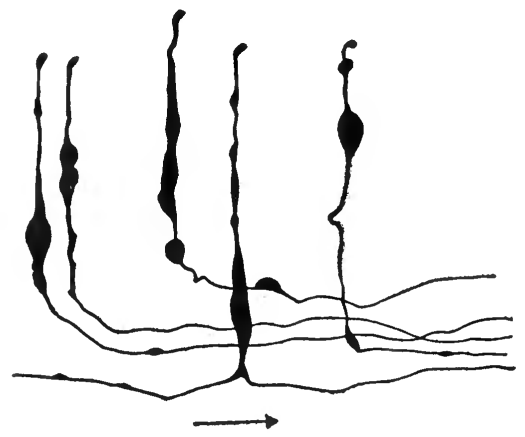

Fig. 18.-Sensory cells of the seaanemone tentacle and their outgrowths; the arrow points toward the base of the tentacle (from Grošelj, I gog). on this definitely directed growth of the fibers. Undoubtedly this conclusion is correct as regards the difference in conductivity in the two directions in the fully developed tentacle, but Parkerapparently fails to recognize the fact that the directed growth of the nerve fibers is not a matter of independent choice, but must itself be determined by physiological conditions. From the time of their appearance as buds, these tentacles show a definite physiological gradient, with differences in electrical potential, susceptibility, reducing power, etc., and the directed growth of the nerve fibers is unquestionably determined by this pre-existing gradient.

While the evidence is by no means complete, all that we have indicates very clearly that the differentia- 
tion of nervous structure is in general localized, first, with reference to the physiological surface-interior relations, and, second, with reference to the axiate relations as represented by the primary or chief body gradients. The earliest appearance and the chief aggregation of nervous structure is localized at or near the high ends of the gradients present in the organism concerned, and the general direction of nervous differentiation and of nerve outgrowth during the earlier stages of development is down the chief body gradients. Later of course the situation becomes always more and more complicated by the localization of new regions of high activity and new gradients, which may determine locally the direction of nervous differentiation and growth, but the sequence of events is orderly and definite.

\section{THE EARLY DIFFERENTIATION OF NERVOUS}

\section{STRUCTURE}

The very early differentiation of nervous structure is another striking feature of ontogeny. In the coelenterates nervous differentiation is so slight that it is difficult to determine when it begins, but in other animals we find that the central nervous system becomes recognizable at least as a region of localized growth at the anterior end soon after the establishment of the primary germ layers and before other definitive organs make their appearance (Figs. I3-I7).

These various facts appear to me to indicate a very close ontogenetic relation between the primary physiological gradients and nervous differentiation, and they afford a physiological basis for various features of nervous development which have heretofore remained 
obscure. If the primary physiological relations in the pattern of the organism are chemical or transportative and excitation and transmission relations arise only secondarily the localization of the nervous system with respect to the gradients, its early development, and the fact that it becomes the chief integrating organ all remain remarkable and completely inexplicable facts of ontogeny, but if it is true that organismic pattern originates in excitation-transmission relations and that the axial gradients are protoplasmic records of such relations, all these facts appear in a new light. The localization, the early differentiation, and the function of the nervous system, which represents the specialized mechanism of excitation and transmission in the organism, appear as the natural and necessary consequence of the existence and physiological nature of the gradients. Moreover, from this point of view nervous structure appears as the primary or fundamental developmental reaction of the more active body regions. The distinction between ectoderm and entoderm precedes its appearance, but the entoderm represents physiologically the lower levels of the chief axial gradient and the nervous system is the first definite and definitive organ to differentiate from the higher levels of the gradient or gradients.

The older conception of ontogeny as a process of construction of a machine which, after construction is completed, begins to function appears less and less satisfactory as our knowledge advances. Living protoplasm is functioning at all times and development is a process of functional construction, that is, beginning with a given structure and function, the continuance of func- 
tion modifies the structural substratum, and this in turn modifies further function and so on. If the effect of functional activity on the structural substratum is ever completely reversible it is only or chiefly in the fully developed organism where a dynamic equilibrium between structure and function is attained or approached. Development represents the progress from a disturbed protoplasmic or cellular equilibrium to an organismic equilibrium, or, as in physiologically isolated regions or physically isolated pieces, the progress from a disturbed organismic equilibrium to a new equilibrium, and the metabolic or physiological gradients constitute the framework or ground plan on which this progress takes place.

The primary germ layers represent the primary regional differentiation with respect to the gradient pattern. They result from the differences in rate of metabolism at different levels of the gradient and constitute the basis for a chemical or transportative relation which arises sooner or later, when ectoderm grows at the expense of the yolk in the entoderm, or of food digested by the entoderm. This transportative relation between ectoderm and entoderm is, however, secondary and the consequence of the differences in rate of activity between them. The nervous system, on the other hand, represents the primary differentiation with respect to the functions of excitation and transmission of the gradients, and as the gradients represent the primary integrating factor in the development of the organism, the nervous system as the differentiated mechanism of this primary integrating function necessarily becomes the chief organ of integration. 
According to this conception the nervous system is the physiological and morphological expression of the excitation-transmission relations, first with respect to the primary or chief physiological gradients, and later with respect to the progressive developmental complications as they arise.

The absence of a nervous system in plants apparently depends upon the inability of plant protoplasm to synthesize molecules sufficiently stable under the existing physiological conditions to constitute the basis of a permanent morphological structure of nervous character, that is, possessing a stable protoplasmic structural substratum and at the same time a mechanism permitting intense dynamic activity. In general, the capacity of plant protoplasm for morphological differentiation is slight as compared with that of animal protoplasm, and plant structure consists very largely of cellulose. Consequently physiological integration in plants retains more or less its primitive character, based on a gradient in physiological activity, physiological dominance of the apical region, the growing tip, is not very effective, and repeated physiological isolation and reproduction, as evidenced by the development and arrangement of multiple axes in the form of branches and various other parts, is a characteristic feature of the life of most plants.

This conception of the ontogenetic origin of the nervous system is purely physiological and involves no assumptions concerning inheritance or evolution. The gradients do not determine the nature of nervous structure but merely provide the physiological basis for the realization of certain of the hereditary potentialities of the protoplasm concerned and the problem of the 
evolutionary origin of those potentialities remains as before. We are primarily concerned with the developmental physiology of the nervous system and the problem of the phylogenetic origin, and evolution of nervous structure is involved only in so far as it can be analyzed in terms of developmental physiology. 


\section{CHAPTER VII}

THE PHYSIOLOGICAL RELATION OF THE CENTRAL NERVOUS SYSTEM TO OTHER PARTS

\section{IN DEVELOPMENT}

PHYSIOLOGICAL INDEPENDENCE AND DEPENDENCE IN DEVELOPMENT IN RELATION TO THE GRADIENTS

Attention has already been called (p. 97) to the fact that the establishment of a physiological gradient determines the existence of a relation of dominance and subordination in which the high end of the gradient, because of its greater irritability and activity, is physiologically dominant over all levels of the gradient within a certain range of distance, which varies with the activity of the dominant region and the conductivity of the protoplasm. Similarly, any level of the gradient is to some extent dominant over more basal or posterior levels and subordinate to more apical or anterior levels. This relation of dominance and subordination appears most clearly in the simpler axiate organisms, both plants and animals, and its significance in ontogeny and in the regulatory development of isolated parts as well as in function has been considered at length elsewhere (Child, I9Iо $a$, I9I I $c, d$, I9I $5 c$, chaps. iv, v).

This relation may be stated not only in terms of dominance and subordination, but in terms of independence and dependence. So far as one region or level of the axis is dominant over another, it is independent of the other. In other words, in so far as a more irritable 
or more active region $A$ becomes a factor in determining through transmission of excitation the physiological condition of another less irritable or less active region $B$, in so far is $A$ independent of $B$. Evidently then the high end of a gradient must be, at least to a large extent, independent of other levels, and in bilateral forms not only must the anterior region be largely independent of more posterior levels, but the median ventral (invertebrates) or the median dorsal (vertebrates), so far as it represents the high end of the symmetry gradients, must be relatively independent of more lateral regions.

Such a relation, if it actually exists, must appear in the order of development and the functional relations of parts along an axis. The so-called law of anteroposterior development is merely a statement of this relation for the longitudinal axis. Development and differentiation begin anteriorly or apically and progress posteriorly or basally because the anterior or apical region, being more intensely active than other regions, is so far as they are concerned self-determining, at least in large measure, and each body level is also to some extent self-determining with respect to more basal or more posterior levels and at the same time to some extent determined by more apical or anterior levels (Figs. 8-I2). Similarly the median region is to some extent self-determining with respect to the lateral, and more lateral levels are to some extent determined by more median. In plants the growing tip arises first in the development of an individual axis, whether stem or root, and is manifestly self-determined with respect to other parts, since it may arise in their complete absence, and other parts are subordinate to and determined by it. 
The apparent independence and capacity for "selfdifferentiation" of different levels or regions of the body in certain animals, e.g., annelids and mollusks, may seem to be completely irreconcilable with the conclusion advanced above, but what the data on "selfdifferentiation" and "mosaic development" actually demonstrate is not the absence of relation between parts but merely the fact that protoplasmic specialization has advanced so far that the regulatory reactions to isolation of parts are limited, and the behavior of the part after isolation is therefore much the same as before. The evidence indicates that conditions of this sort are due simply to early cytoplasmic specialization rather than to the fundamentally "mosaic" character of ontogeny.

THE PHYSIOLOGICAL INDEPENDENCE OF APICAL REGION AND HEAD IN EXPERIMENTAL REPRODUCTION

In normal ontogeny the whole body is potentially present, and objection to the conclusions stated above may be raised on the ground that, even where differentiation is not perceptible, it may still be sufficient at lower levels of the axis to influence the development of higher levels. But further evidence in support of these conclusions concerning independence and dependence, some of it demonstrative, is obtained from the regulatory development of isolated pieces from many of the lower animals. In the first place it has long been known that in the development of pieces isolated from the body of various species of hydroids, flatworms, and annelids, the apical or anterior region of the pieces gives rise directly to an apical structure or head, irrespective of 
the level of the body from which the piece was taken. In Planaria, for example, pieces taken from regions near the original head and those from more posterior levels (Fig. I9, $a$ and $b$ ) behave in essentially the same way in this respect. Although the anterior end of piece $b$ represents a body level which is normally separated from the head by the whole pre-pharyngeal and pharyngeal region, a new head develops directly from the anterior end of $b$ as well as from that of $a$ and the body regions corresponding to those between the head and the level of $b$ in the original animal arise later by the reorganization of the tissues of $b$ posterior to the new head (Fig. 22). Moreover, this reorganization never takes place unless a head does develop or begin to develop. In the earthworm and other annelids which are not too highly specialized to give rise to new heads from postcephalic body levels the developmental process is the same in these respects. In fact, in all cases where isolated pieces give rise to new individuals the development of the new apical end or head is the first new differentiation at the anterior end, irrespective of the body level represented by the piece. That is to say, this process of so-called regulatory development is essentially similar to embryonic development in that differentiation of the new individual begins at the apical or anterior end and proceeds basipetally or posteriorly. In a piece like $b$ (Figs. I9-22) the new head is "out of place" when it arises, i.e., it adjoins parts which in the normal animal are far removed from the head. The normal relations are re-established by the redifferentiation into the various intermediate regions of parts of the piece posterior to the new head. The significance 


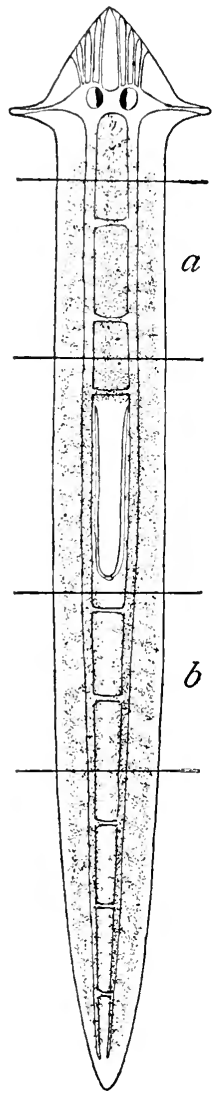

19
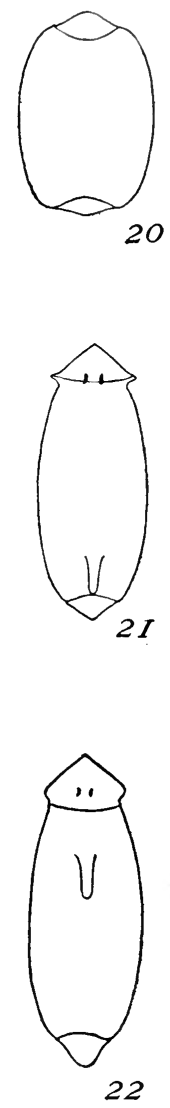

Figs. 19-22.-Planaria dorotocephala: reconstitution of pieces from different levels: Fig. I9, diagrammatic, showing general plan of central nervous system and alimentary tract, $a$ and $b$, two regions from which pieces are taken; Fig. 20, early stage of reconstitution in both $a$ and $b$; Fig. 2I, later stage from $a$; Fig. 22, later stage from $b$ (Figs. I9-2I from Child, I9I $5 c$ ). 
of these facts has been discussed elsewhere and it has been shown experimentally that the development of the regions normally intermediate between the head and the level represented by the piece is determined by the presence of the developing head (Child, I9Ir $b$, I9r $5 c$, pp. IO2-I7). Similarly, the development of a new posterior end at the posterior end of the isolated piece is determined by the regions anterior to it and cannot occur independently of them, though it may occur in the absence of a head (Figs. 8-r2). The development of the new head, however, actually occurs in spite of the rest of the piece and may under certain conditions be more or less completely inhibited by the rest of the piece (Child, I914d, $e$, г9r6b, I920).

The most striking evidence for the physiological independence of the apical end or head is found in the regulatory development of very short pieces. Such pieces give rise to apical or cephalic structures alone, all other parts of the body being entirely absent. Short pieces of Tubularia stem (Figs. 23-29) from any level may give rise to single or biaxial whole hydranths or partial hydranths depending on its length, and short pieces of the planarian body may give rise to single or biaxial heads (Figs. 30-34). The frequency of biaxial structures in these short pieces is due to the fact that the short piece, representing a very small fraction of the polar axial gradient, is almost apolar, and in many cases the exposure and the presence of the wound at the two ends is sufficient to determine two opposed gradients and so a biaxial development. These apical and cephalic structures which develop in the complete absence of other parts may be complete and normal in all respects 
so far as can be determined. The single or biaxial planarian heads may contain fully developed cephalic

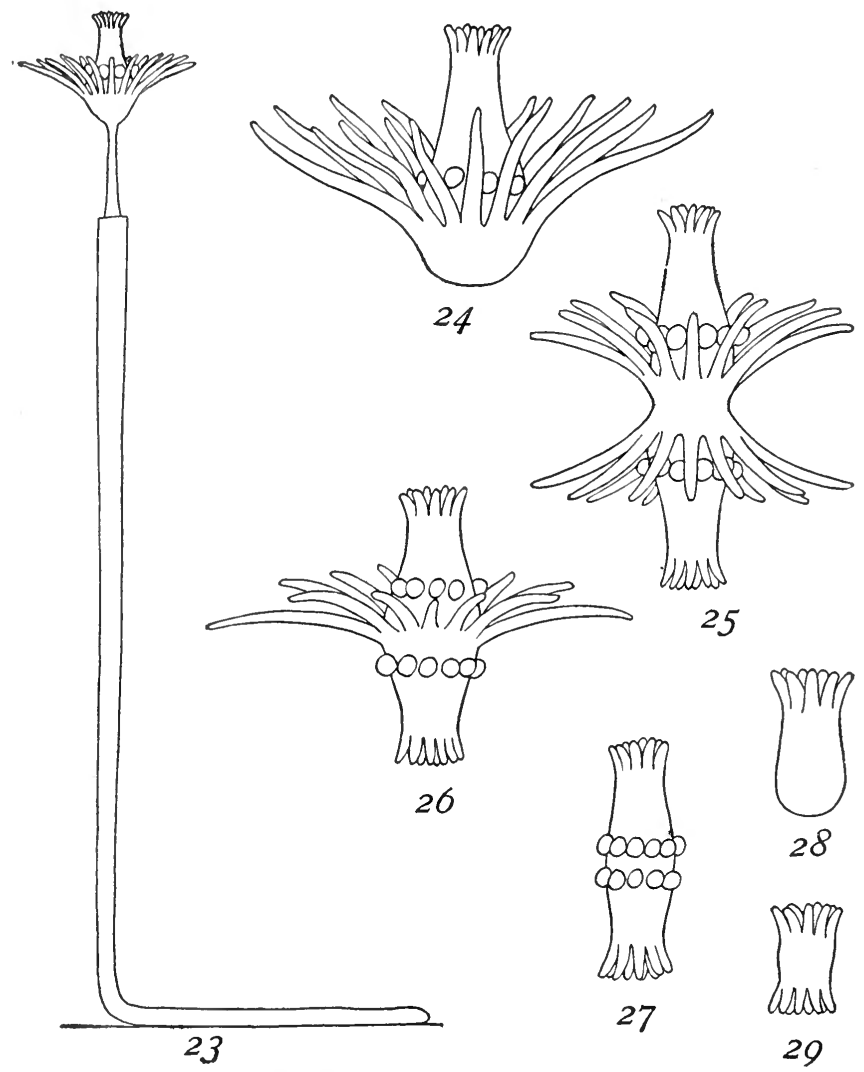

FIgS. 23-29.-Tubularia: Fig. 23, an unbranched individual; Figs. 24-29, single and biaxial apical structures which develop from short pieces of the stem (from Child, I9I $5 c$ ).

ganglia. In such cases there can be no doubt that these apical or cephalic structures have arisen in complete independence of other parts of the body. Thus 
far no other region of the body has ever been seen to develop from pieces in this way. All other levels of the body along the polar axis develop only in relation to more apical or more anterior regions. In Planaria, for example, we never find small pieces transforming completely into a new posterior end, although pieces may
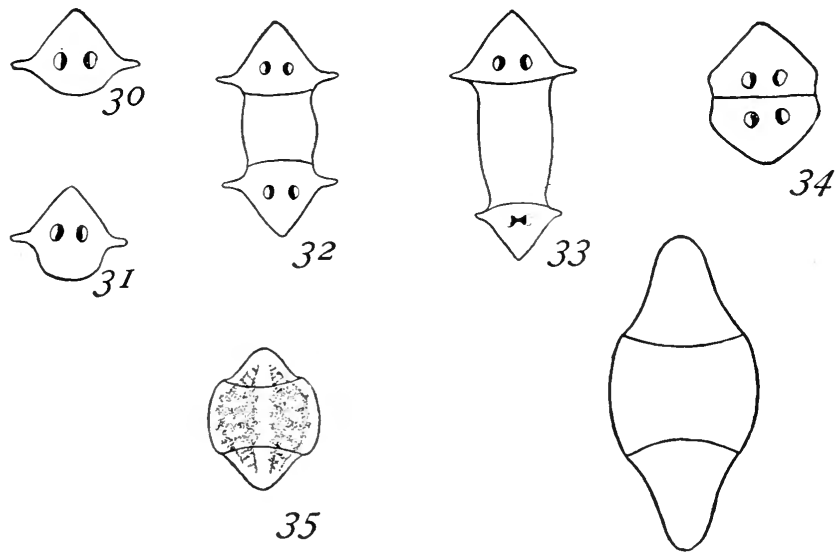

36

Figs. 30-36.-Partial structures from short pieces of Planaria: Figs. 30-33, monaxial and biaxial heads from P. dorotocephala; Fig. 34, biaxial heads from $P$. simplicissima; Fig. 35, biaxial tail from $P$. dorotocephala; Fig. 36, biaxial tail from $P$. simplicissima (Figs. 30-33, 35, from Child, $1915 c)$.

give rise to new posterior ends on one (Figs. I I-I 2 ) or on both cut surfaces (Figs. 35-36) in the absence of a head.

THE RELATIVE INDEPENDENCE OF THE NERVOUS SYSTEM. IN DEVELOPMENT

All the data both from normal ontogeny and from experimental or regulatory reproduction indicate that the apical region or head, at least as regards the earlier 
developmental stages, is independent of other parts. In all except the simplest animals the cephalic central nervous system is the primary organ of the head region. If the head region is self-determining with respect to other parts, certainly the same must be true in large measure for the cephalic nervous system.

In this connection it is of interest to note that Flexner (1898) and Keiller (I910) have shown that in the regenerating head of Planaria the ganglia may develop quite independently of the cut ends of the nerve cords in the piece and the connections are established later by outgrowth from the new ganglia rather than from the nerve cords. The development of the connections between the new ganglia and the old cords in certain abnormal types of head in Planaria also points to the same conclusion (Child and McKie, I9II). Moreover, Goldfarb (I909) removed the nerve cord from several segments at the anterior cut end of pieces of earthworm and found that the head and the ganglia developed normally in such pieces, and the nerve cords grew posteriorly from the new head into the old parts, although some regeneration in the anterior direction from the end of the old cord occurred. In this case it is demonstrated beyond question that the ganglia in the regenerating head develop independently of other parts of the nervous system in the piece.

There seems then to be no escape from the conclusion that, at least in the lower animals and as regards its earlier stages, the apical or cephalic portion of the central system develops independently of subapical or postcephalic regions. Its physiological dominance and its independence are simply two aspects of the same 
physiological situation and both are consequences of the fact that this portion of the central nervous system develops from the high end of the primary gradient, i.e., from the region of the body in which activity is most intense during the earlier stages.

The experiments of Waelsch (I9I4) and Fischel (I9I4) on birds and Amphibia indicate that in these forms also the earlier embryonic stages of the central nervous system arise independently of other parts. Peripheral parts undoubtedly do exercise some determining influence on the later differentiations in the central nervous system of vertebrates, as is shown by the experimental work of Shorey (I909) on birds and Amphibia and numerous data on the retardation or inhibition of development of central organs in the absence of peripheral function in man and mammals. This, however, is unquestionably a secondary condition and associated with the decrease in "steepness" or decrement in the chief axial gradients with the advance of development. In earlier stages, and in many of the lower organisms throughout life, the difference in irritability or metabolic condition is so great that transmission is chiefly or wholly in one direction, i.e., down the primary or chief gradients because most excitations cannot run very far up the gradients without disappearing as excitations in the higher metabolic level (see pp. 97-99). With the ontogenetic and phylogenetic increase in conductivity along the gradients and the consequent decrease in decrement, excitation may be transmitted to greater and greater distance up a gradient, and peripheral parts or lower levels of a gradient become increasingly important factors in determining differentiation and physiological 
condition at higher levels. In other words, the primitive type of axiate physiological integration is similar to the autocracy in social integration, but in the development and evolution of the organism, as in social development and evolution, there is a gradual approach toward democracy.

While the evidence is less extensive, the data of normal development and of experiment indicate that the ontogenetic relation of the postcephalic regions of the central nervous system to regions lateral and dorsal (invertebrates) or ventral (vertebrates) to them is similar to the relation of the cephalic to the postcephalic regions. These postcephalic regions of the central nervous system apparently arise from the high ends of the symmetry gradients (Figs. $\mathrm{I}^{-}-\mathrm{I} 7$ ), and so far as these regions are dominant they must be primarily independent of the parts which they dominate.

\section{THE SIGNIFICANCE OF THE FACTS}

It is a fact of considerable theoretical interest not merely to the biologist but to the psychologist as well that the cephalic region of the central nervous system arises from that region of the body which primarily is most intensely active and most completely independent of other parts. In fact the development of the central nervous system and particularly of its cephalic portion represents that physiological and morphological reaction of animal protoplasm which is least complicated by physiological correlation with other parts and most directly related to external exciting factors. The central nervous system arises from the higher levels of the chief axial gradients in the organism, and these represent 
the dominant regions of the physiological gradients which are present before the nervous system appears. I have endeavored to show that these gradients are in the final analysis responses to the differential action of external factors upon living protoplasm, and if we admit this it follows that the localization and the general pattern of the centralized and cephalic nervous system is determined physiologically by a response of protoplasm to environmental action upon it. Moreover, the central nervous system is the chief organ of excitation and transmission and the chief organ of physiological integration, because it represents the working out in ontogeny and phylogeny of the primary organismic integrating factors, the excitation-transmission relations.

The special characteristics of nervous structure as distinguished from those of other organs are of course determined by the hereditary constitution of the protoplasm concerned. As I have already pointed out, the physiological gradients merely provide a framework or plan as a basis for the realization of certain hereditary potentialities, and with evolutionary changes in the hereditary mechanism, this physiological plan, and therefore the localization, structure, and relations of the nervous system, undergo alteration. But there can be no doubt that the earlier stages of the development and differentiation of the nervous system are in large measure independent of other organs, and I have endeavored to show that this independence is due to the fact that the nervous system, more than any other organ, is the expression of the primary physiological factors of organismic pattern and integration. 


\section{CHAPTER VIII}

THE NERVOUS SYSTEM IN RELATION TO SEGMENTATION

The bodies of the annelids and arthropods among the invertebrates and the bodies of all vertebrates are made up of segments, metameres, or somites, and the problem of the origin and the evolution of this segmentation or metamerism has ranked as one of the great problems of speculative morphology, while its physiological aspects have received but little attention.

Without attempting a survey of the various theories of segmentation it may be noted that they involve two different conceptions of the segment and of the process of segmentation. According to the one conception the process of segmentation is essentially a process of agamic reproduction, the segment being equivalent to a new zooid, but instead of becoming a complete new individual and undergoing separation, it is co-ordinated or integrated with the head region and other segments into a new individuality. In short this theory conceives the process of segmentation as a modification of certain processes of agamic reproduction, such as the repeated transverse fissions which occur in certain Turbellaria and give rise to temporary chains of zooids.

The other conception regards segmentation as originating in the reduplication of certain internal organs, and most of the theories based on this conception hold that the process is primarily localized in the 
mesoderm and that the reduplications in other parts are secondary.

It is evident that as a physiological process segmentation, as well as many other repetitive developmental processes, must involve a rhythmical repetition in physiological conditions of some sort. Ritter and Johnson (Ritter, I909, I9I9 [II, 95-I07]; Johnson, I9Io; Ritter and Johnson, I9II) have called attention to the significance of these repetitive processes as evidence of law and order in organisms and to their bearing on the problem of integration. I have shown that various processes of agamic reproduction in both plants and animals result from physiological isolation (Child, r9roa, I9гіd, I9г5c, chap. v; Child and Bellamy, I9r9) and have suggested that the physiological conditions determining the reduplications of parts, or meristic series, as Ritter terms them, are essentially similar, except that they concern more or less specialized parts and their repeated development rather than entire organisms. If this suggestion is correct there is little fundamental difference, so far as the physiological conditions are concerned, between the two chief theories of segmentation. In the one case the process of development of a new segment presumably begins in the ectoderm, since this is primarily the most active layer, and later involves the other layers; in the other case the process begins in the mesoderm and secondarily involves other layers.

The theories of segmentation have been primarily morphological rather than physiological. When we consider segmentation as a physiological process, as it of course must be in every case, it is difficult to find an 
adequate physiological basis for the theory of the mesodermal origin of segmentation. Even if reduplication of mesodermal organs did occur, it is difficult to understand how or why it should have led to the segmentation of ectodermal structures and even of the nervous system. Moreover, many of the lower invertebrates, e.g., various Turbellaria, show reduplication of certain mesodermal and of entodermal parts without any indication of actual segmentation in muscles, nervous system, or ectoderm in general. The theory of mesodermal segmentation regards such forms as the precursors of segmented animals, but what evidence we have seems to me to point to a different conclusion.

In the polychaete annelids, so far as they have been studied in this way, the earliest stages of development show a single apico-basal gradient in susceptibility, but at a stage much earlier than the beginnings of segmentation in the mesoderm an ectodermal region of high susceptibility, indicating high physiological activity, appears in the posterior part of the developing larvae (Fig. 37) and from this region the ectoderm of the trunk arises (Child, I9I $d$ ). The appearance of this region resembles physiologically the appearance of a second zooid in the posterior region of Planaria (Child, I9 I $d$, I9г $3 b)$, and it seems probable that it is determined by some degree of physiological isolation in much the same way as in Planaria, except, of course, that it is much earlier in development. This region is already established as a growing region when the mesoderm consists of only a few cells and the formation of successive segments from it is evidently a repeated reproductive process. 
We may conceive the process somewhat as follows: the physiological isolation of this posterior region of the developing organism results first in increased cell division and growth, i.e., it becomes or remains less specialized instead of continuing to differentiate, like other parts. This is the same process that we find in other cases of physiological isolation. Second, in this mass of embryonic cells the development of a new individual begins, but in this case the individual becomes a segment instead of a complete organism. Its failure to develop a head may be due either to incomplete isolation from the head region or to the presence of some degree of specialization in the cells concerned which makes them incapable of giving rise to a head.

In this connection it may be pointed out that among the lower invertebrates, e.g., the flatworms, the first limi-

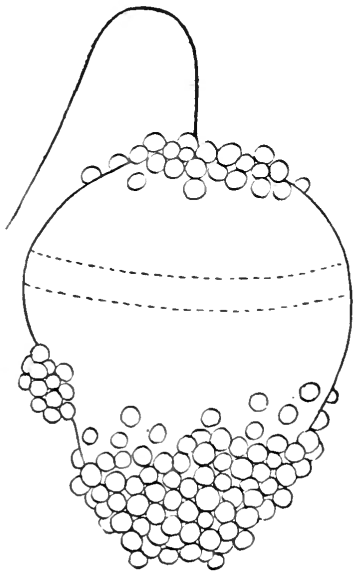

Fig. 37.-Early larval stage of Chaetopterus, a polychaete annelid, showing the primary apical and the secondary posterior regions of high susceptibility as they appear when the cells begin to swell and separate in various agents; at this stage the mouth is developing and also appears as a region of high susceptibility. tation in the power of regeneration of pieces of the body with advancing specialization is the loss of capacity to give rise to a new head. In the triclad turbellarian, Planaria, for example, pieces above a certain length from any level of the body are able to regenerate heads 
as well as other parts and become complete new individuals (Figs. I9-22). In the polyclad Turbellaria, however, pieces from levels posterior to the head are unable to develop new heads, though they regenerate all other parts. In certain other forms, e.g., the triclad, Dendrocoelum (F. R. Lillie, Igor) and various oligochaetes (Hyman, I9I6), the capacity to regenerate a head exists in the more anterior body regions and disappears at a certain level. In one of the rhabdocoeles, Stenostomum, the capacity to regenerate a head exists in the younger asexual stages but disappears when the animals become sexually mature. Moreover, in embryonic development the head arises earlier than other parts, i.e., when the cells are physiologically younger and their rate of physiological activity higher, and it is localized in the most active region of the organism. And finally, the earlier stages of head development are more susceptible to the action of inhibiting agents than those of other parts of the body. All these facts indicate that a higher rate of metabolic activity in the cells concerned is necessary for the development of a head than for that of other parts, i.e., in order to produce a head the cells must be physiologically younger, or must arise from a higher level of the axial gradient and therefore undergo more complete dedifferentiation and attain a higher rate of activity, than is necessary for the development of other parts.

Returning to the process of segmentation it appears then not improbable that the cells of the posterior growing region in annelids have already become specialized to such an extent that they are capable of giving rise only to incomplete headless individuals. In any 
case the segment resembles a headless individual of Planaria (see Figs. I I, I2). The process of growth and differentiation is repeated for each segment, but instead of becoming free and developing heads, like the zooids of Planaria, the segments are integrated into a new whole, probably chiefly through the development and increase in conductivity in the nervous system.

The early appearance of a posterior growing region involving primarily the ectoderm and giving rise to the segments has been demonstrated not only in the polychaetes but in the oligochaetes (Hyman, I9I6), various Crustacea and fishes, and Bellamy (I9rg) has shown that it appears in the frog at an early stage (Figs. 38, 39) and that through the action of inhibiting agents its localization can be altered so that it arises nearer the apical pole of the egg than normally, much as the anterior end of a new zooid can under inhibiting conditions be localized nearer the head in Planaria and various other forms (Child, I9II $d$ ). In unsegmented animals a definite posterior growing region of this sort is usually not present, except in cases of agamic reproduction, though eren in these forms more or less growth may occur at the posterior end as they increase in length. These facts show at least that the posterior growing region is determined in the ectoderm earlier than in the mesoderm and, I believe, increase the probability that segmentation as a physiological process begins in the most active layer, the ectoderm, and that it is physiologically a process resembling various processes of agamic reproduction.

That the central nervous system of segmented animals shows more or less definite indications of being 
itself segmented is a fact of common knowledge. In the less highly specialized segmented invertebrates a bilateral pair of ganglionic enlargements of the ventral nerve cord, or a single ganglionic mass with more or less
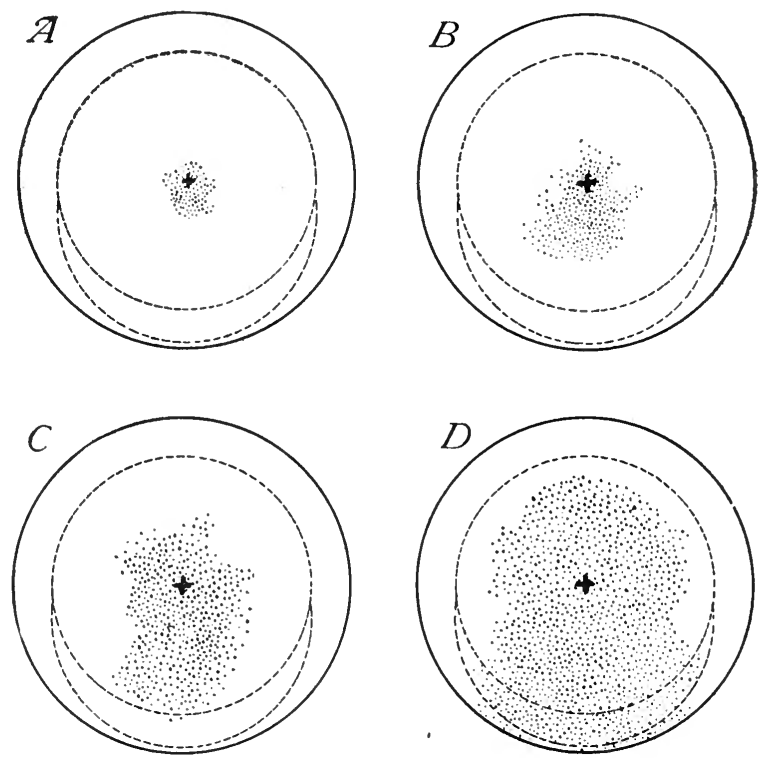

FIG. 38.-Unsegmented egg of frog, viewed from apical pole, showing susceptibility gradient to temperature of $0^{\circ} \mathrm{C}$. The stippled areas represent areas of disintegration, the cross-marks the apical pole, the dotted circle the boundary between pigmented and unpigmented regions, and the dotted crescent the outline of the gray crescent, as they appear on the lower hemisphere (from Bellamy, rgrg).

distinct double bilateral structure, appears, but the various modifications of segmental structure in the different groups are accompanied by modifications of ganglionic arrangement. This is particularly the case as regards the head region which, in the higher invertebrates, 
apparently consists of a number of more or less completely fused segments, and in the vertebrates of a still larger number. Even in the oligochaete annelids Hyman (I9I6) has found indications in the behavior in
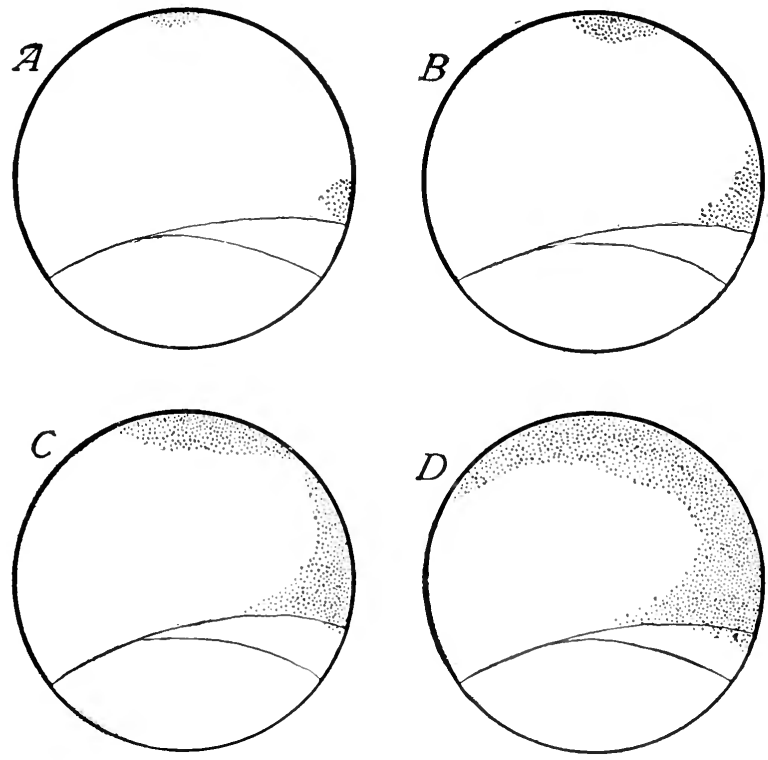

FIG. 39.-Stages of disintegration of frog embryo during late cleavage, in side view; the secondary posterior region of growth is already appearing as an area of high susceptibility in the equatorial region (from Bellamy, 1919).

regulatory development of a number of anterior segments, characteristic for each species, that they constitute a more or less definite head region.

The problem of the segmentation of the vertebrate head has been widely discussed and an extensive literature exists which cannot be considered here. The 
segmentation of the cephalic, and in fact of the whole central nervous system of the vertebrate, appears in the adult in the arrangement of cranial and spinal nerves and spinal ganglia and to some extent in certain parts in the arrangement of centers or nuclei of the nerves. Concerning the embryonic development of this segmentation and its relation to the segmentation of other parts there has been much difference of opinion, centering chiefly about the question whether the segmentation of the nervous system is primary or secondary, i.e., whether segmentation is primarily an ectodermal or general reproductive process or a mesodermal process. Like the evidence on segmentation in general the evidence concerning the segmentation of the vertebrate nervous system seems to me to support the former alternative. The discovery by Locy (I895) of distinct neuromeres in very early stages of the embryonic development of vertebrates, and the later work along this line by Hill (I900) indicate very clearly the occurrence of some sort of repetitive process in the primordium of the central nervous system as it arises (Figs. 40-44). Neal (I898, I9I8) among others has criticized this evidence and has attempted to show that the neuromeres of early stages do not correspond to the final segmentation, but in spite of these and other criticisms the physiological significance of Locy's neuromeres appears beyond question as indicating a rhythmical repetitive process of some sort along the anteroposterior axis in very early stages.

The neuromeres of the head region appear of course in that portion of the embryo developing from the primary region of growth which has its anterior end at 

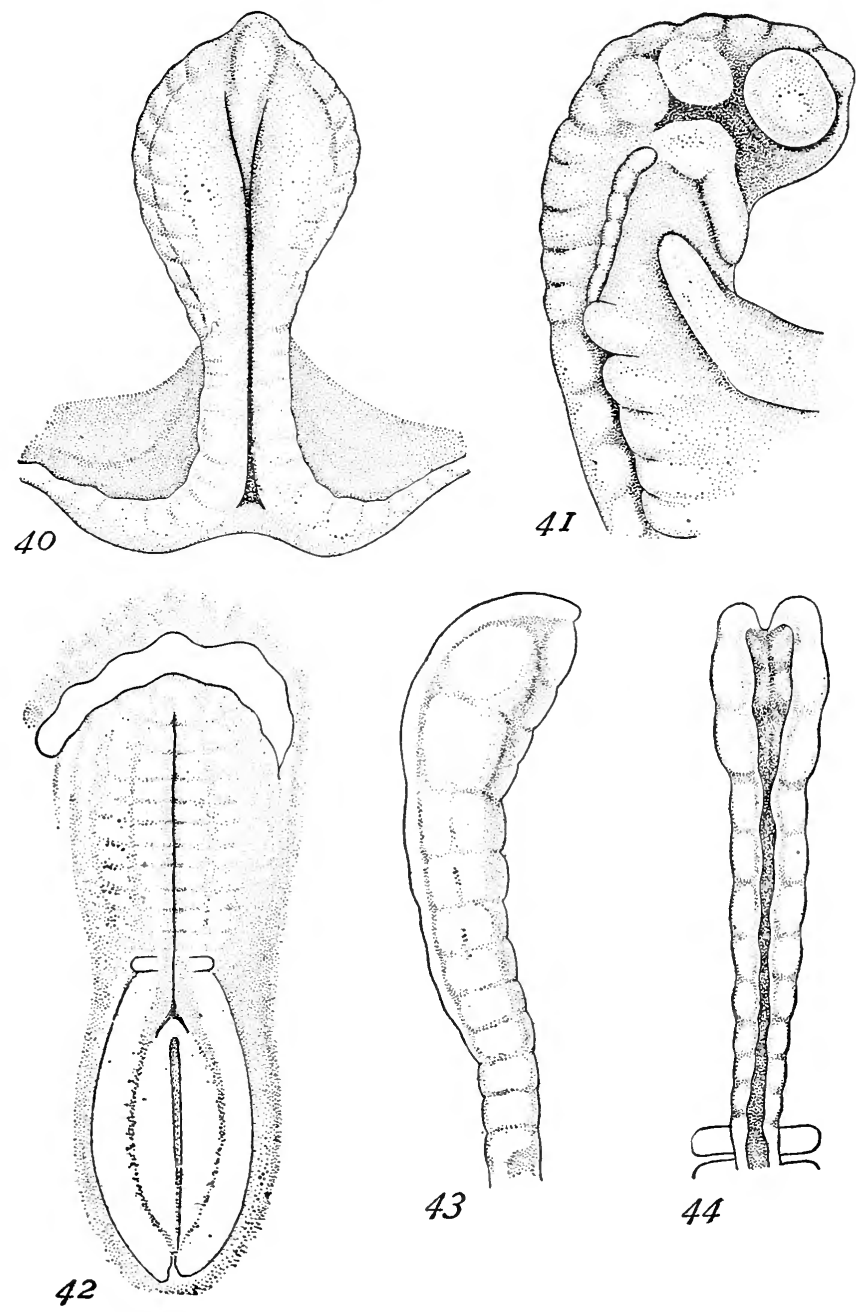

FIGS. 40-44.-Embryonic segmentation of the central nervous system of vertebrates: Figs. 40, 4I, Squalus acanthias, dogfish, surface view of young embryo and side view of nervous system of later stage (from Locy, I895); Figs. 42-44, chick; Fig. 42, dorsal view of embryo with one somite, twenty-one hours old, neural segments traceable, not only across wide neural groove but some distance laterally into adjacent tissue; Fig. 43, dorsal view of anterior portion of neural tube of embryo twenty-two hours old; Fig. 44, right surface view of same neural tube as Fig. 43, showing that segments encircle neural axis (from Hill, I900). 
or near the apical pole of the egg. That is to say, they are wholly anterior to the secondary region of growth from which the postcephalic segments arise, and it has been suggested above that the appearance of this secondary growing region is the result of physiological isolation. The presence of the cephalic neuromeres indicates the occurrence of a rhythmic, repetitive process in the nervous primordium even in this anterior region. If we regard the cephalic neuromeres as true segments, we account in evolutionary terms for their appearance by assuming that they represent the remaining traces of an ancestral segmented condition of the cephalic region. So far as I am aware, however, the problem of the physiological process involved has not been considered. Obviously it is a true repetitive or reproductive process in the cephalic primordium. There is little prospect of obtaining actual experimental knowledge concerning this process until physiological technique is much more highly developed than at present, but it may be suggested that the increasing physiological activity and its progress in the posterior direction in the embryonic primordium are the chief factors in the repetitive process of neuromere formation. As each level of the primordium attains a certain degree of activity it becomes to some slight extent physiologically isolated from more anterior levels and some slight amount of localized growth occurs, but the degree of isolation is so slight that it does not proceed very far.

According to this point of view the origin of the secondary posterior growing region in the embryos of segmented animals and the development of the trunk 
segments from it are physiologically merely a continuation in later developmental stages of a process essentially similar to that which in the very early stages gives rise to the cephalic neuromeres. Moreover, the very early appearance of indications of segmentation in the primordium of the nervous system, which is ectodermal, favors the conclusion that segmentation is primarily an ectodermal rather than a mesodermal process and this conclusion again is in accord with the fact that the ectoderm is physiologically the most active body-layer in the earlier embryonic stages and the layer in which the earliest differentiations appear. The mesodermal theory of segmentation is based primarily on morphological speculation rather than on physiological experiment, and it seems to me to offer little prospect of interpretation or synthesis of the various facts as they now stand, and particularly of the very early participation of the ectoderm and its earliest definitive differentiation the nervous primordium in the repetitive process. The facts apparently indicate that segmentation begins in the ectoderm and that the processes in this the most active and most advanced body layer affect conditions in other layers. The segmental ganglion is then the primary definitive organ of the segment as the cephalic ganglion is of the individual. 


\section{CHAPTER IX}

\section{NERVOUS CENTRALIZATION AND CEPHALIZATION IN EVOLUTION}

The terms "centralization" and "central nervous system" possess of course a morphological and a physiological significance. Morphologically, centralization is the process, both ontogenetic and phylogenetic, of localization and aggregation of the chief mass of nervous tissue in a particular region of the body. Speaking physiologically, centralization represents integration, and the central nervous system is really the integrative nervous system, as Sherrington (I906) has so beautifully demonstrated. The term "cephalization" refers to one particular aspect of centralization, viz., the condition or process of integration with respect to the longitudinal axis. The point to which $I$ wish to call particular attention is that these terms refer not merely to a morphological localization or aggregation, but to a physiological condition and process, and also to an evolutionary process. Morphological centralization and cephalization in any particular case are at best only the indication or record in structure of the degree of integration attained. Moreover, a high degree of nervous centralization or cephalization may exist in comparatively simple forms with relatively slight degree of integration as compared with the higher animals, e.g., ascidians, rhabdocoele and polyclad Turbellaria in which the central nervous system is entirely in the head. 
The aggregation of the whole central nervous system in a single ganglion or pair of ganglia, as in these forms, does not of course mean that these animals have attained a higher degree of integration than the vertebrates, but merely that the degree of integration is higher than would be the case in similar forms with a more diffuse system. In the adult ascidian the function of the ganglion as an integrating organ is certainly not highly developed, but the ascidian tadpole represents a rather high degree of centralization and the high degree of morphological centralization in the adult appears to be rather the consequence of the earlier course of development than directly associated with conditions in the adult. The degree of integration in the rhabdocoeles and polyclads is unquestionably much higher than in the triclad Planaria, where the central nervous system includes not merely the cephalic ganglia but the longitudinal cords and apparently some of their branches (Fig. I9, p. I 22). The degree of integration corresponding to a particular degree of centralization or cephalization in a particular group depends upon the hereditary potentialities of the protoplasm concerned as regards the development of nervous structure and function.

\section{CENTRALIZATION IN RELATION TO AXIATION}

According to current views, the nervous system is primitively diffuse and uncentralized and centralization appears only in the course of evolution. I have endeavored to show that the localization and differentiation of nervous structure, and the development of nervous function constitute only a part of a developmental process, that they must depend upon physiological conditions 
already present in the developing organism before the nervous system as a specialized organ appears, and that the physiological gradients as the earliest distinguishable features of organismic pattern are fundamental factors in the process which determines the localization and development of a nervous system.

From this viewpoint the nervous system appears as a morphological and physiological expression of the gradients in the developing organism, and both its early differentiation and its function as an organ of excitation and transmission indicate its intimate relation to these fundamental factors of organismic pattern. If we accept this view, we can expect to find an uncentralized nervous system only in animals which are completely anaxiate and which possess only a surfaceinterior pattern, and it is difficult to conceive how such a system could contribute in any way to organismic integration. All receptors of such a system would be equally susceptible to excitation, conduction would be diffuse and equal in all directions, and the resulting integration would be temporary, and probably in many cases only partial as in Amoeba, and would soon give place to another integration with another receptor as the dominant region. As a matter of fact, some degree of organismic and even of axiate pattern and integration already exists before the nervous system appears (p. 242) and the development of the nervous system merely continues the process. If strictly an axiate animals exist they do not give rise to nervous systems. All animals in which any visible differentiation of nervous structure appears are to some extent axiate before nervous differentiation occurs, though in the sessile coclenterates, 
and particularly in the sponges, such axiation is not very highly developed. We must expect therefore to find at least some degree of structural or functional axiation, even in the most primitive types of nervous system existent. Even in the sessile coelenterates we do find various indications of nervous axiation and integration (pp. 243-44) and in the medusae nervous axiation is much more marked. In radial forms, e.g., coelenterates and echinoderms, the different radii are more or less equivalent. There is no indication of any persistent dominance of any particular radial axis either in the medusae or in most of the sessile coelenterates. In the medusa, Cassiopea, that particular marginal sense organ which at any given time gives rise to impulses most rapidly, i.e., which has the highest rhythm and is therefore presumably the most active, is for the time being dominant, and similar radial relations apparently exist in hydroids and most actinians. In some actinians, e.g., the Cerianthidae, there are, however, some indications of a differential or preferential relation among the radii in certain reactions, and in the echinoderms we find this preferential relation or functional dominance of certain radii, in all degrees of development even involving marked structural bilaterality of the body wall in certain groups.

Nervous differentiation is not coincident with the beginnings of axiation in animals but represents a later stage in the development of axiation, consequently the nervous system shows some degree of axiate pattern even in its most primitive forms; in other words, wherever differentiation progresses far enough to give rise to any distinguishable nervous structure some degree of structural or functional axiation or centralization is probably 
always present. The physiological basis of nervous centralization exists as soon as axiate pattern is established, that is, more or less definite axiate excitation-transmission relations are determined before the appearance of specialized nervous structure, and when such structure appears its localization and general pattern are determined by these still more fundamental features of pattern.

\section{PROGRESSIVE CENTRALIZATION AND CEPHALIZATION} IN VARIOUS GROUPS

Starting from the slight degree of centralization or axiation of the nervous system which is present in its most primitive clearly recognizable forms, we find many indications of progressive centralization and cephalization, which is centralization with respect to the longitudinal axis. In most of the great phyla or in some of their groups the evolutionary process appears more or less clearly. Even within the triclad Turbellaria different degrees of cephalization appear and, as already noted (p. I42), the rhabdocoeles and polyclads show a higher degree of cephalization than the triclads. In the mollusks again conditions range from the "stepladder" nervous system of the amphineura through the ganglionic system of pelecypods and gastropods to the highly cephalized system of the cephalopods. The annelids, on the other hand, show but little progressive change in these respects, although certain data of regeneration indicate that a number of anterior segments definite for the species, but differing in different species, are more or less integrated with the prostomium to form a head region (Hyman, I9I6). In various groups of the Crustacea, in the insects and to some extent in the 
arachnids the process of cephalization appears in one form or another. To what extent these processes occur in the echinoderms is uncertain, but the holothurians probably represent a somewhat more advanced cephalization than the other groups. And finally the progress of nervous cephalization from the lower to the higher vertebrates is perhaps the most significant feature of vertebrate evolution. The evolutionary progress of nervous cephalization is accompanied by various other changes in form, proportion and relations of parts, particularly in the segmented animals, in which the fusion of segments at the anterior end of the body, the decrease in total number of segments, and the increasingly intimate physiological relations of the segments in general are characteristic features.

Concerning the physiological basis of these changes we know as yet practically nothing, but it is evident that the changes represent modifications of the axiate pattern with respect to the longitudinal axis, and if the gradient is the physiological basis of axiate pattern, these evolutionary changes must be interpreted physiologically in terms of gradients. Undoubtedly the form or curve of a physiological gradient, its slope or steepness, is different in different kinds of protoplasm, and undoubtedly also the capacity of protoplasm to develop a high conductivity has increased in the course of evolution. The experimental data at hand do not afford a basis for exact quantitative measurement or comparison of the gradient curves in different organisms, but they do indicate that the gradients undergo changes in the course of evolution as well as in ontogeny. The evolutionary change means merely that as the constitution 
and hereditary potentialities of protoplasm are altered in the course of evolution, however we suppose such alteration to occur, the form, slope, or curve which a physiological gradient will take and the rate or intensity of physiological activity at its high end may also undergo change. In other words, the reaction of the protoplasm to the conditions giving rise to a gradient, or in the presence of a persistent or inherited gradient, must undergo modification in the course of evolution, like other protoplasmic reactions. In whatever terms we may prefer to interpret the problem of cephalization with respect to inheritance and evolution, I believe the physiological basis of the differences in different forms will be found to consist in the differences in the character of the physiological gradients in the different protoplasms.

The localization of the "central" nervous system in more or less distinct regions of the body in the pelecypods and gastropod mollusks indicates that the physiological conditions concerned in the localization of nervous structure in these forms are somewhat different from those in most other invertebrates. As regards the gradients in development some data have been obtained for certain gastropods, and these indicate that the primary polar gradient of early embryonic stages is more or less completely obliterated in larval stages and that in place of it several regions of high physiological activity arise, the foot, the visceral mass, the mantle folds, and the branchial region. It may be suggested that the peculiar ganglionic form of the nervous system in these groups is associated with these physiological facts, the differentiation of nervous structure being 
associated and localized in a definite way in relation to regions of high activity. In this connection it may also be suggested that certain ganglia outside the central nervous system in other forms arise in connection with secondary regions of high activity.

THE "STEPLADDER" TYPE OF NERVOUS SYSTEMI IN THE INVERTEBRATES

The relation of the central nervous system to the symmetry gradients is definite and clear except in some of the lower invertebrates. In most of the bilateral invertebrates in which longitudinal nerve cords form part of the central nervous system, viz., most flatworms, annelids, and arthropods, the postcephalic longitudinal portions of the central nervous system are ventral, in the vertebrates dorsal, and except in some of the lower invertebrates they arise as a bilateral organ at or near the median line. Various lines of experiment and observation indicate that median and ventral in most bilateral invertebrates and median dorsal in the vertebrates, represents the high region of the symmetry gradients and is, at least during earlier stages of development, a region of more intense physiological activity than the regions on either side of it (pp. 26, 58).

In some of the lower invertebrates, however, the relation between the longitudinal nerve cords and the axial gradients is not so clear. In Planaria, for example, and in most other Turbellaria the nerve cords lie some distance lateral to the median line on each side (Fig. I9, p. I22), in the cestodes the chief nerve cords are near the lateral margins of the proglottides, and in the nemerteans also the nerve cords are more lateral than median. 
Even in Planaria it has not yet been possible to determine the primary symmetry gradients with certainty because of the lack of early embryonic stages, but in all the Turbellaria examined the ventral region is, at least primarily, a region of higher activity than the dorsal, and while the lateral margins of the body may develop high activity in the ectoderm in later stages the growth of new tissue from the cut ends of pieces and various other facts suggest that in the parenchyma the median ventral region is more active than the lateral.

There are some reasons for believing that dorsiventrality is the first step in the development of bilateral symmetry in some of these forms and that the distinction between median and lateral is of later origin. If this is true, the localization of the nerve cords in these lower vertebrate groups may represent the high ends of the symmetry gradients, i.e., the cord may arise where the high activity of the ventral region begins to decrease laterally and dorsally. Where the whole ventral region is alike and the gradient begins laterally the cords may be lateral, and as the width of the ventral active zone decreases they may arise nearer the median line. This interpretation is offered merely as a suggestion, although there is some evidence in support of it. Until we know more concerning the early embryonic stages in these forms, definite conclusions are not possible. But whatever the actual conditions in these lower forms may prove to be, it appears to be true in general for the higher invertebrates and the vertebrates that differentiation of nervous structure is primarily and predominantly localized at the higher levels of the chief 
body gradients. The evolutionary progress of centralization and cephalization from the primitive more or less diffuse localization of nervous structure in relation to the general body surface is one aspect of the evolution of axiate pattern.

At present, however, we can do little more than note the relation between the physiological gradients and nervous localization. How it comes about physiologically that the higher levels of these gradients give rise primarily and predominantly to nervous structure is a problem in the physiology of localization and differentiation like that pertaining to any other organ. We can see that the origin of the chief aggregation of nervous structure from that region of the embryo which is primarily most active, most sensitive, and physiologically dominant represents a continuous sequence of physiological processes, but our present knowledge of living protoplasm does not permit us to analyze this sequence into its physicochemical terms.

\section{THE POSITION OF PERIPHERAL RECEPTORS}

The apparent migration of the cell bodies of peripheral receptors from various regions of the body surface toward or into the central nervous aggregation constitutes another aspect of the evolutionary process of centralization. Primitively these cells differentiated in the general epidermis (Fig. I8) and gave rise to nerve fibers which grew toward the central nervous system and were afferent in function, but in the vertebrates most of these cells arise in close relation to the central nervous system and send fibers peripherally as well as centrally. The olfactory epithelium alone retains the epidermal 
origin. It is evident that in the more primitive conditions the localization of these cells is determined primarily by the surface-interior, rather than the axiate pattern. The whole ectoderm as that part of the organisms most directly related to the external world may be regarded as potentially nervous. It is obvious, however, that in the course of evolution the axiate pattern has become predominant over the general surface-interior pattern. The whole hereditary mechanism concerned in reproduction provides in more and more definite ways as evolution proceeds for the establishment of such axiate pattern in the egg or for its persistence through the reproductive process. At the same time the surface-interior pattern, while still evident in many ways in the higher forms, becomes less and less important as a primary factor in differentiation. Doubtless the phylogenetic development of protective coverings of the egg and early embryonic stages is concerned in these changes, the greater degree of protection of the body surface by egg coverings, etc., from the direct action of external factors in the early developmental stages of higher forms, determining that the general surface-interior gradients shall be less definite and not so far above the general level of intrinsic activity. With this phylogenetic change, the evolution of axiation, the relation of nervous differentiation to the regions of most intense metabolism in the embryo appears in the shifting of the region of differentiation of the receptor cells from the general body surface toward the high regions of the axiate pattern and they, like the motor neurons, send fibers peripherally, i.e., down the chief axial gradients. 
From this point of view this process of shifting in position is physiologically similar to certain features of the process of cephalization, e.g., the phylogenetic migration of segmental ganglia toward, and their aggregation in, more anterior regions. The localization of nervous structure in any particular organism is primarily an expression of the localization of the regions of higher rate of the fundamental dynamic activities in that organism and the progressive growth and differentiation of nervous structure is primarily from the regions of more intense to those of less intense activity. The statement that the whole ectoderm is potentially nervous, a statement which has been repeatedly made, means in terms of this conception that wherever the fundamental physiological activity of the ectoderm cells is sufficiently intense there nervous structure will arise, provided the protoplasm is capable of recording this activity in permanent structural differentiation. The evolutionary progress of nervous centralization and cephalization signifies that, as the axiate pattern becomes more and more predominant over the primitive surface-interior pattern, it plays a larger part in determining the localization of the physiological conditions which underlie the development of nervous structure. And since these conditions are evidently associated with the higher levels of metabolic or oxidative activity in protoplasm the development of nervous structure becomes more and more definitely localized in the high regions of the axial gradients.

\section{CONCLUSION}

The phylogenetic changes in localization and aggregation of nervous structure are of course not independent 
of the changes in other parts of the organisms. We see clearly enough that the organism has evolved as a whole, not as a mosaic of independent parts, but the changes in the nervous system are among the most fundamental and significant changes which have occurred. As long as we regard the nervous system as representing physiologically a new factor superadded at some period in evolution to the primitive organismic system, the phylogeny as well as the ontogeny of the nervous system and its relation to other parts remains a complete mystery, and we can merely record and not interpret the facts. But phylogeny as well as ontogeny must sooner or later be interpreted into terms of developmental physiology and potentialities of the hereditary mechanism of the various protoplasms. The conception which I have attempted to develop in this book provides at least a working hypothesis for further attack upon the problems of developmental physiology, not only as they concern the individual but also in their phylogenetic aspects. It must not be forgotten, however, that this hypothesis is purely physiological and is based primarily on physiological evidence. It concerns the actual physiological conditions under which potentialities of the hereditary mechanism or action system are realized. The problem of the hereditary mechanism remains, although $\mathrm{I}$ believe that increasing knowledge concerning the physiological conditions under which hereditary potentialities are realized must teach us something concerning the physiological basis of these potentialities. 


\section{CHAPTER X}

\section{THE PROBLEM OF NEURON PATTERN}

It has been shown in preceding chapters that a definite relation exists, as regards localization differentiation, and the course and degree of centralization and cephalization, between the nervous system as a whole and the physiological pattern of the organism. Moreover, it has been pointed out that the problem - of the nature of this relation is first of all a problem of developmental physiology and that it must be solved in terms of the physiology of organismic pattern. And finally, an attempt has been made on the basis of a physiological conception of organismic pattern to throw some light on this problem.

\section{THE NEURON}

The current conception of the cellular constitution of the nervous system is that, except in its most primitive forms, it is made up of distinct cells, the neurons, which usually give rise to outgrowths of two sorts, the axons or neurites and the dendrites (Figs. 45-53). In such a nervous system functional continuity from cell to cell is established during the course of development. It has been demonstrated in many cases and is believed by many to hold for all that this continuity consists in a contact relation between axon and dendrite, axon and cell body or dendrite and cell body of different cells with persistence of the cell membrane between the parts in contact. This region of contact between two cells is 
termed the synapse, and a nervous system consisting of distinct neurons connected by means of synapses is known as a synaptic nervous system. In some of the lower invertebrates and in certain organs of the higher animals, however, the nervous system consists of a network or reticulum of branching cells forming a syncytium, i.e., direct protoplasmic continuity apparently exists from cell to cell through the branches, without the intervention of the membrane characteristic of the synaptic type of connection. The cellular elements of such a nervous network have been called by Parker protoneurons (Fig. 66, p. 203). The protoneurons usually show little or no definite morphological axiation or polarity, but the forms of neuron most characteristic of both invertebrates and vertebrates show very definite axiate patterns, though a wide range of variation in form occurs.

The problem of the polarity and morphological pattern of the neuron is of interest from various points of view: First, as regards its origin, the question whether it is inherent in the neuroblast or is determined in relation to correlative factors is of the same importance as in other cases of axiate pattern. Second, if this polarity is of correlative origin, the question of the nature of the factors concerned is of fundamental significance, since the neuron patterns are essential factors in determining nervous structure and interrelations. Third, the question of the relation between the visible morphological polarity and the functional polarity of the neuron as an element of the nervous system is an important aspect of the problem of nervous function. 
The problem of the nature and origin of neuron polarity has received comparatively little attention from neurologists, and in view of the fact that experimental data are lacking, any consideration of the problem at this time will doubtless appear to some as a completely futile undertaking. Neuron polarity and pattern are, however, essential features of nervous pattern, and it is scarcely possible in a discussion of the origin of nervous pattern to ignore the problem of neuron pattern. In its most highly developed form this pattern represents a specialized type of intracellular axiate pattern which appears in a very definite and orderly manner, though with extreme variation and complication as regards axial direction and spatial relations. We must at least attempt to discover whether there is any basis for believing that this pattern is similar in nature and origin to other types of axiate pattern. Any exhaustive consideration is of course impossible, but it is desired to call attention to certain facts which appear significant and to suggest certain interpretations which may possibly point the way to an experimental attack on the problem.

THE GENERAL MORPHOLOGICAL CHARACTERISTICS OF NEURON PATTERN

Our knowledge of the morphology of the neuron is based upon the extensive investigation of Golgi, Ramon y Cajal, Ehrlich, Retzius, Bethe, and many others, and an extensive literature has grown up, from which is evident notonly the bewildering variety of neuron pattern as regards detail but also certain general characteristic features. As reference to any of the general works on the histology of the nervous system will show, the following 


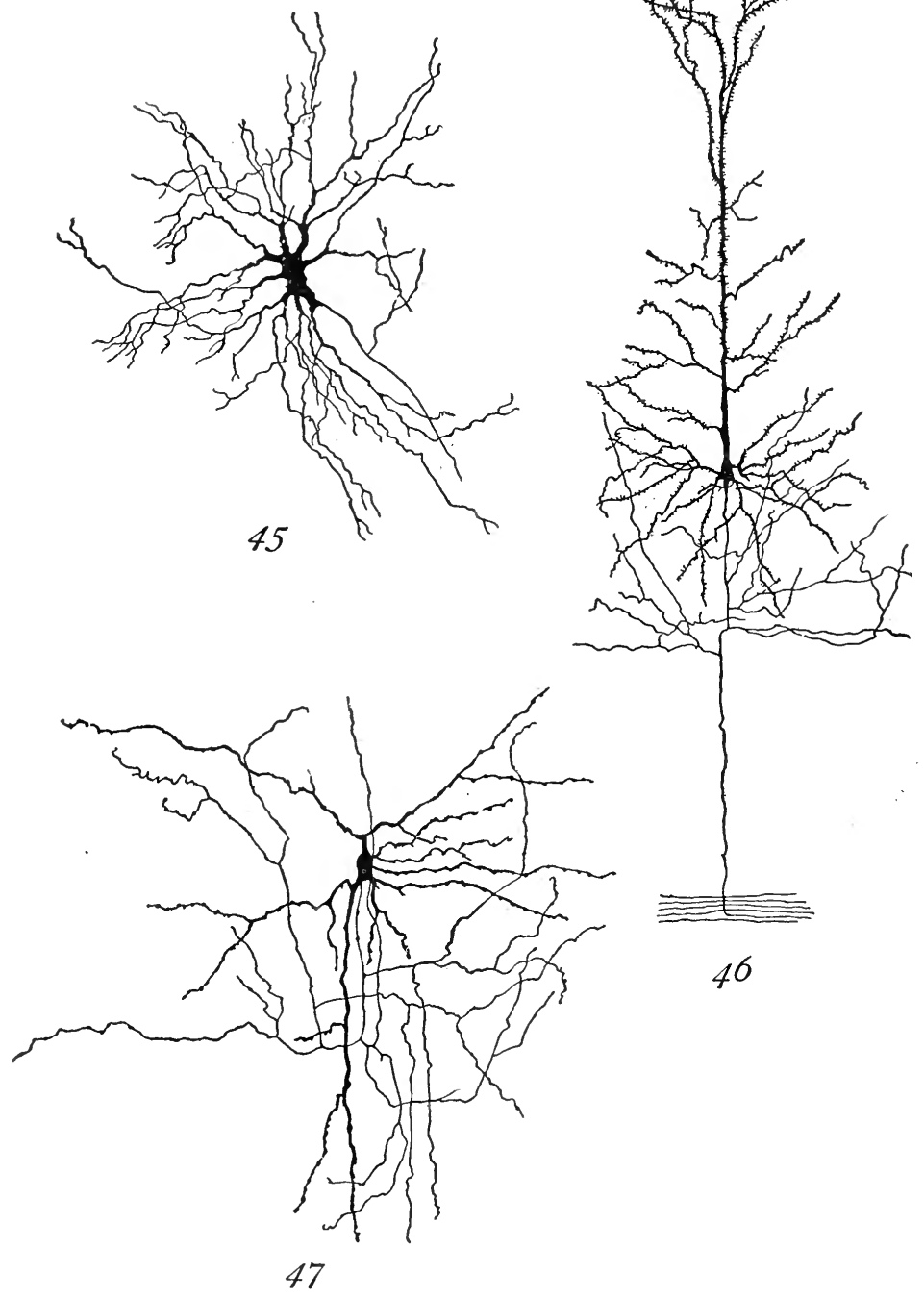

FIGS. 45-47.-Fig. 45, motor cell of ventral horn of spinal cord from human embryo $30 \mathrm{~cm}$. long (from Barker, I gor, after von Lenhossek); Fig. 46, pyramidal neuron of cerebral cortex of rabbit (from Cajal, I909); Fig. 47, neuron with short, branching axon, from cerebral cortex (from Cajal, I909). 
brief survey serves merely to call attention to some of the more common and characteristic aspects of neuron pattern.

The most characteristic feature of the differentiation of the neuron from the neuroblast is the appearance of outgrowths of the cell body which often develop an extremely complex system of branches, resembling the branching of the multiaxial plants. In most vertebrate neurons the two kinds of outgrowths, axon and dendrites, are clearly distinguishable. The axon is slender and of uniform diameter over all or most of its length and possesses a characteristic structure differing from that of other parts of the cell. The axon may extend for a long distance from the cell body with no, or very few, branches, as in many motor cells (Fig. 45), or it may give rise to lateral branches, the collaterals, in the immediate vicinity of the cell body (Fig. 46). In other neurons it may break up into branches and terminate within a short distance from the cell (Fig. 47). It may terminate in a complex system of branches, the terminal arborization, near or far away from the cell body, or it may end with little or no branching. The relations of axons to dendrites of other neurons also differ widely in different cases. In some the axon or some of its branches form extensive spirals about the dendrite while in others the approach is more or less direct. Usually a neuron gives rise to only one axon, though dioxonic and even polyoxonic neurons occur, and usually also the axon is the primary outgrowth of the cell. But some neurons remain without axons.

The dendrites, on the other hand, are structurally very similar to the cytoplasm of the cell body and are 


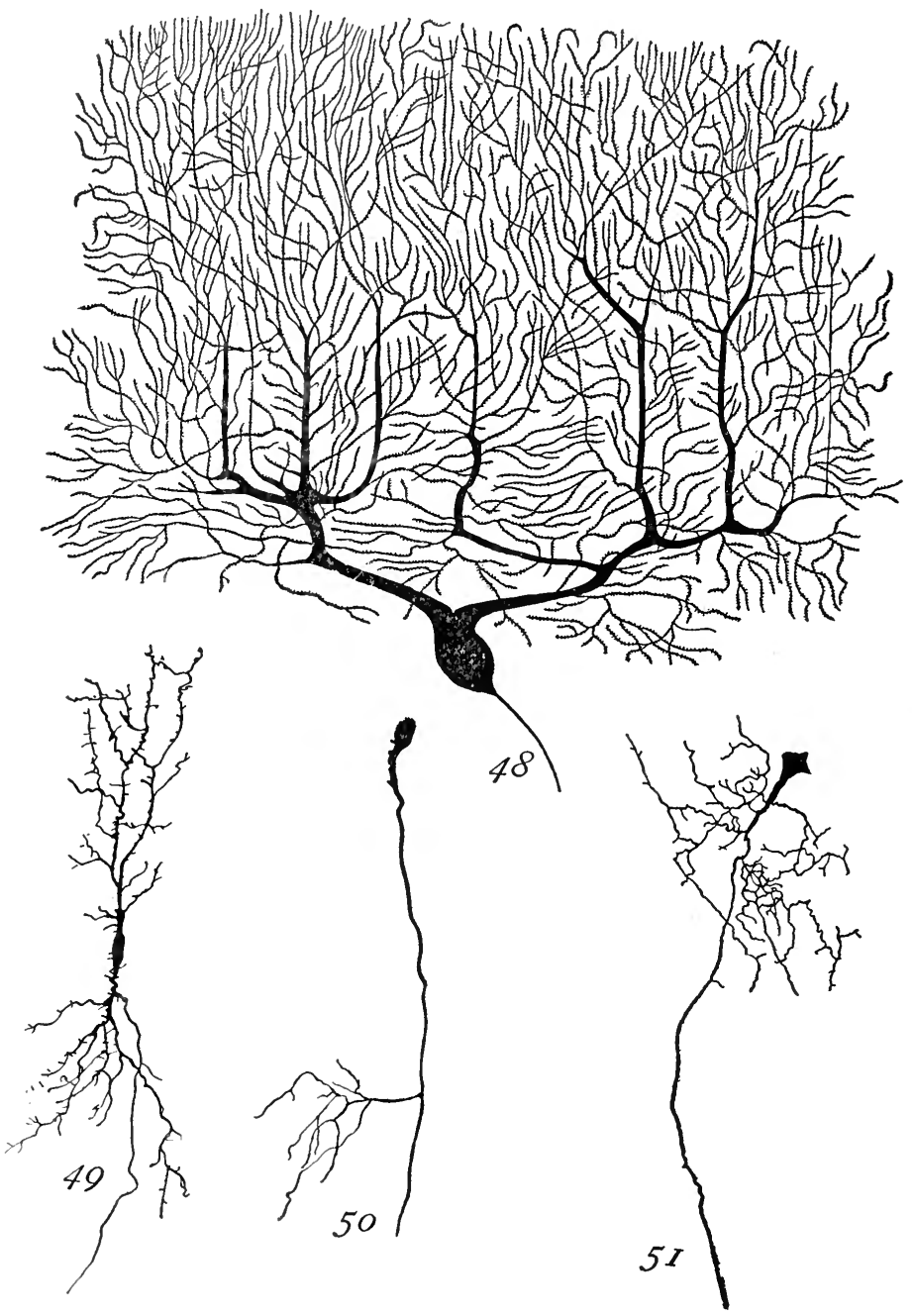

FIGS. 48-51.-Fig. 48, Purkinje cell from human cerebellum (from Stöhr); Fig. 49, fusiform cell from anterior corpus quadrigeminum of rabbit (from Cajal, 1909); Fig. 50, monopolar neuron from mesencephalic nucleus of fifth cranial nerve (from Cajal, I909); Fig. 5I, neuron of smaller gustatory type from carp (from Herrick, I905). 
obviously extensions of it. As the term "dendrite" implies, they usually branch repeatedly, giving rise to treelike complexes, and in general the diameter of the dendrites decreases from the bases of the main trunks to the ultimate branches (Figs. 45-53). Moreover, the neuron usually gives rise to several or many dendrites at different points of its surface. In some neurons, e.g., the pyramidal cells of the cerebral cortex, a chief dendrite is distinguishable, arising from the opposite end of the cell and growing in the opposite direction from the axon (Fig. 46), and some neurons may show only a single dendrite trunk. In still other types of cells the dendrites may arise in two chief groups, or from two main trunks at opposite ends of the cell (Fig. 49), or several groups or trunks may develop, and in some cases the axon takes its origin from some region of a dendritic outgrowth. Again, numerous dendrites arise in some neurons from all regions of the cell surface and extend and branch in all directions (Fig. 45). At the other extreme are neurons without dendrites, for example, peripheral sensory neurons and some central neurons (Fig. 50). Among the lower vertebrates and the invertebrates a common type of invertebrate neuron pattern is that with a single outgrowth from which both dendritic branches and an axon arise (Figs. 51, 52, 53). Anaxonic neurons also occur frequently among the invertebrates.

As regards its developmental history, neuron pattern shows still further complications. The final relations of axon and dendrites, particularly of the latter, are often quite different from their relations in earlier stages, and it is only by following the course of development that we can hope to gain any light concerning the factors 
involved. In the pyramidal cell of the cerebral cortex (Fig. 46) the course of development according to Cajal

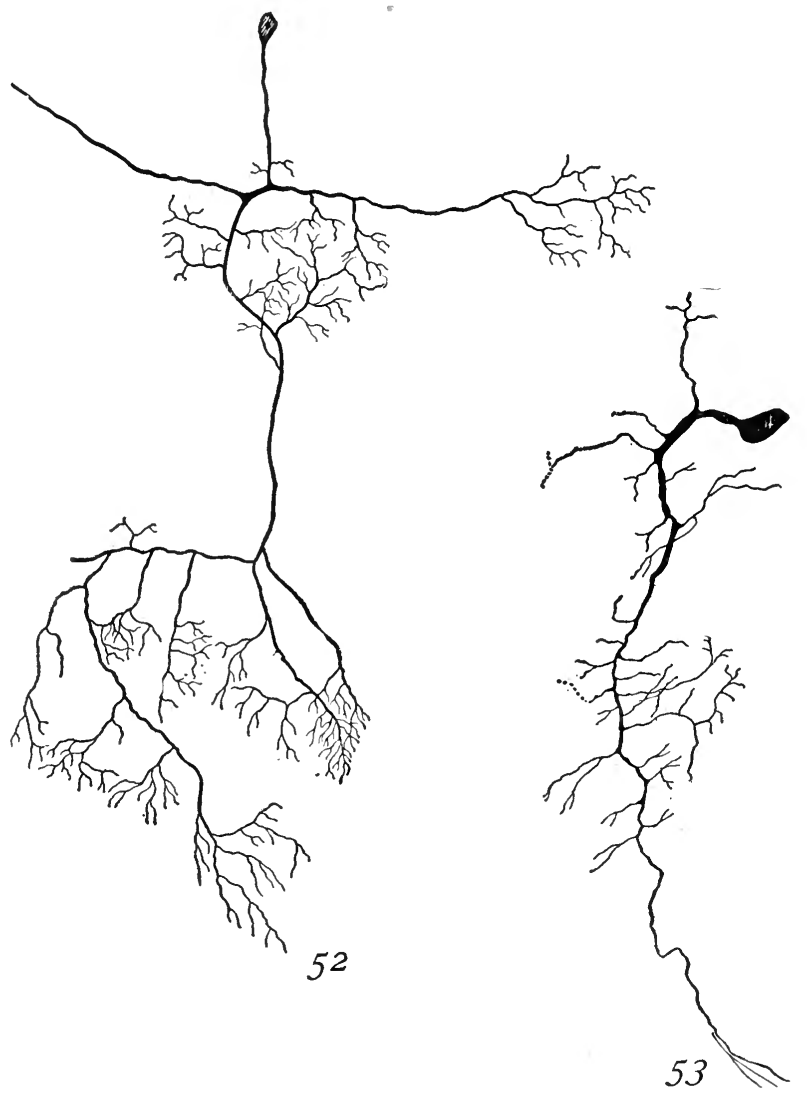

Figs. 52, 53.-Neurons: Fig. 52, neuron from cephalic ganglion of crab, Carcinus maenas (from Bethe, 1897); Fig. 53, neuron from ventral cord of earthworm (from Retzius, 1892).

(Fig. 54, A-E) is as follows: the primary outgrowth, the axon arises on that side of the cell away from the 
external surface of the cortex and its growth is directed away from the surface $(A)$. The chief dendrite appears at the opposite end of the cell and its growth is directed toward the surface $(B, C)$; as the axon elongates,

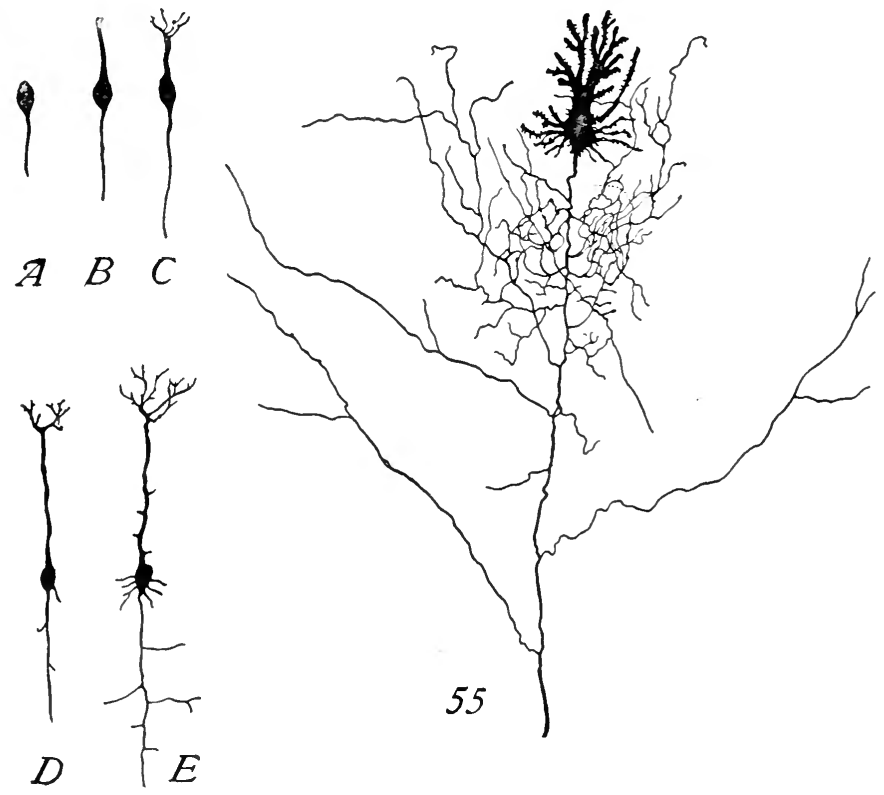

\section{4}

FIGS. 54, 55.-Fig. 54, development of the pyramidal neuron (from Cajal, r9II); Fig. 55, Purkinje cell from cerebellum of new-born infant (from Cajal, I9Ir).

collateral branches arise from it and grow more or less vertically to its direction $(D)$; and still later dendrites arise from various parts of the cell surface and grow in various directions $(E)$. Taking the cell at the stage $B$ or $C$ it appears that axon and dendrite are localized 
at opposite poles of the cell, and it might be supposed that some factor in the cell environment to which the two outgrowths react in opposite ways, e.g., the one positively, the other negatively, determines the direction of their growth. Later, however, the axon gives rise to collaterals which grow in a direction more or less vertical to that of the primary axon $(D)$, and finally the cell gives rise to dendrites which grow in various directions $(E)$. The apparently simple orientation and opposed directions of growth of the earlier stages are much complicated later and no simple interpretation appears possible. Taking all the facts into account it is impossible to doubt that the pattern of such a neuron arises in some sort of relation to its environment, but it is evident that the environmental factors, or the reactions of the cell or its parts to the environment, or both environmental and intracellular factors, undergo alteration and complication during development.

Again, in the fully developed Purkinje cell of the human cerebellum (Fig. 48) the axon arises from the side of the cell away from the external surface of the cerebellar cortex and passes inward, i.e., away from the surface, but usually not vertically to it. The collateral branches of the axon form more or less obtuse angles with the axon direction. The dendrites apparently arise at or near the opposite pole of the cell from the axon and their more basal portions extend parallel to the surface, while their branches show an orientation apparently with respect to the surface, which becomes more and more definite with approach to it.

In the new-born child, however, the Purkinje cell has according to Cajal the form of Figure 55. The chief 
dendrites arise opposite the axon, but numerous other dendrites are present on all parts of the cell surface and extend in all directions. Evidently the situation at this stage is very different from that in the mature neuron. The primary dendrites are more or less definitely oriented, but many others exist which show no uniform orientation. These latter are evidently temporary and are resorbed in later stages. Cajal distinguishes a period of outgrowth and one of regulation and ordering of the dendrites. Here, as in the pyramidal cell, very considerable changes occur in the course of development, both in localization and orientation of the dendrites.

The developmental stages of the axon of the Purkinje cell also show extensive changes. In the new-born infant the axon shows numerous, much-branched collaterals near the cell body as well as a number of longer and less-branched collaterals. In later stages extensive resorption of the shorter, highly branched collaterals occurs, and in the adult only the longer, lessbranched outgrowths remain. This neuron evidentally gives rise to many outgrowths which are only temporary and play no part in the final morphological and functional relations of the neuron. Apparently a selection among the outgrowths takes place during development and presumably on a functional basis, that is, those outgrowths which play an essential part in the functional relations of later stages persist, while those which are not thus functionally active disappear, like larval organs and other temporary structures in the development of organisms. It is evident, however, that the appearance of these outgrowths must be determined by certain 
physiological conditions and the persistence of some and the resorption of others by certain other conditions.

In many other neurons dendrites arise from all parts of the cell surface and grow in all directions without orientation at any stage (Fig. 45). In some cases in which the axon of the mature neuron arises from a dendritic outgrowth, this outgrowth is secondary and the axon developed originally directly from the cell body. The spinal ganglion cell in early stages shows two outgrowths localized at opposite poles (see Fig. 60, p. I86), but later the bases of the two outgrowths approach each other and unite, and the cell gives rise to a single secondary outgrowth from the end of which the two primary outgrowths extend in opposite directions. In some spinal ganglion cells in mature condition this secondary outgrowth is not single in its origin, but the cell gives rise to two or more outgrowths which branch and anastomose inside the connective tissue capsule which surrounds the cell, but finally unite to form the single outgrowth and in other cells several regions of branching and anastomosis appear in the course of this outgrowth.

Frequently axons or dendrites on reaching a certain point in their course show a sudden and definite change in direction as if they had come under the influence of some environmental factor acting in a direction different from that to which they had previously been reacting. For example, the longer dendrites of the pyramidal cells of the cerebral cortex grow vertically toward the surface, but as they approach it they branch and the branches become parallel to the surface (Fig. 46). In the mature Purkinje cell of man the chief dendrites 
extend more or less parallel to the surface, while the secondary branches are in general vertical to it (Fig. 48). Axons in many cases show similar changes in direction, sometimes very abrupt. Again, the dendrites of certain cells may be at first unoriented but later, as the axons of certain other neurons grow into their vicinity, they may become definitely oriented with respect to these and grow toward them.

Other variations and complications in the form, arrangement, and developmental changes of the outgrowths of the neuron might be described almost indefinitely, but those mentioned suffice to call attention to the bewildering complexity of the facts and the difficulties apparently involved in any attempt at physiological analysis of neuron development. In addition to this problem of external morphology and development the neuron presents various other physiological problems, e.g., the origin and function of the internal structural features, the Nissl substance and the neurofibrils, the physiological relations of different portions of the neuron, both as regards nutrition and maintenance, as indicated by the data of degeneration and regeneration: and as regards functional conduction of impulses. For the present, however, we are primarily concerned with the question of physiological polarity or axiation and the development of the external morphology.

\section{NEURON PATTERN AS A CASE OF AXIATE PATTERN}

Neurons are very commonly distinguished as unipolar, bipolar, or multipolar, according to the number of outgrowths, though the terms "uniaxial," "biaxial," and "multiaxial" are perhaps preferable. There are of 
course in general two types of axial outgrowths, the axons and dendrites, which differ in time of appearance, degree of differentiation, and in part at least in function. While we have no demonstrative experimental evidence for the conclusion, there is every reason to believe that the neuron outgrowths represent physiological gradients

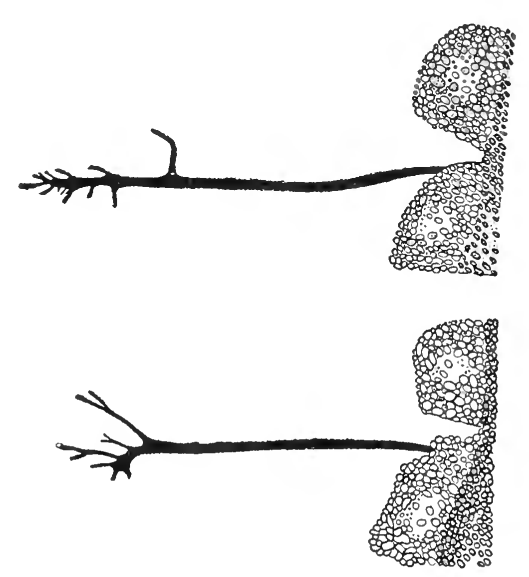

FIG. 56.-Two views, taken fifty minutes apart, of same nerve fiber, growing from group of embryonic spinal cord cells into the lymph of tissue culture (from Harrison, I908). during the period of their development. The axon outgrowth certainly possesses a growing tip which shows evidences during the growth period of greater physiological activity than other parts of the outgrowth (Fig. 56). While the dendrites do not usually show so definite a growing tip as the axon, their growth form, order of branching, and changes in direction of growth show very clearly that growth is primarily terminal in each dendritic axis, although secondary growth in thickness may occur in other parts. In short, all the facts at hand indicate that both axons and dendrites are primarily physiological gradients like other physiological axes in organisms.

It is impossible to proceed very far in the examination of nervous tissue prepared by the Golgi method. or any 
other which shows the external morphological neuron pattern, without being impressed by the resemblance between the usual types of neuron pattern and those of the multiaxial plants and multiaxial animals, such as the hydroids. Many neuron patterns, for example, are much like tree patterns, and different modifications of neuron pattern are at least as clearly distinguishable as the forms of different species of trees. Resemblances of this sort do not necessarily signify identity or even similarity of constitution in the individuals or protoplasms concerned, but I believe they do at least suggest similarity in axiate pattern, i.e., in the relations of physiological gradients in the different protoplasms. It is difficult, in fact, not to believe that similarities so striking as those between neuron pattern and tree pattern, for example, mean something physiologically as regards the relations between the constituent axes and their integration into the whole individual. The hereditary constitution of the protoplasms of neurons and of trees is doubtless very different, and the environmental factors to which the plant is reacting are obviously in large part or wholly different from those to which the neuron protoplasm reacts. Notwithstanding these differences the physiological relations of the axial gradients in the two types of individual may be more or less similar and may determine similar types of growth pattern.

THE POSSIBLE FACTORS CONCERNED IN THE AXIATION OF THE NEURON

The axon is a highly specialized outgrowth whose chief function is evidently conduction. It probably has little or no direct nutritive relation with its environment 
except possibly in earlier stages of its development. Not only its growth but its maintenance depends upon its connection with the cell body, as the experiments on degeneration and regeneration have demonstrated. There can be little doubt, however, that the dendrites are important nutritive organs as well as conductors of impulses. In all probability they are primarily nutritive and continue this function after they become conductors. The axon arises at a definite region of the cell and usually grows in a definite direction for at least some distance, but the dendrites very commonly, though not in all neurons, arise on various regions of the cell surface, often on all sides of it and grow in all possible directions, at least during earlier stages. In some neurons, however, such as the pyramidal cell of the cortex (Fig. 46), a primary dendrite develops on the opposite side of the cell from the axon and grows at first or continuously in a definite direction (Fig. 54). In such cases other later dendrites may show other and various directions of growth.

So far as we are able to conceive the conditions in the developing nervous system, there are apparently only two sorts of conditions in the environment of the developing neurons which can possibly determine the localization or the direction of growth of either axon or dendrites. The one of these is chemical, the other electrical.

The attempt to interpret the localization and directions of outgrowth of axon and dendrites as a response of some sort, whether chemotactic in the strict sense or not, to chemical substances in the environment of the cells meets with various difficulties. In the first place, 
the presence of the chemical substances to which reaction is supposed to occur and the proper concentration gradients must be assumed. Moreover, in a neuron like the pyramidal cell (Figs. 46, 54) the reaction of axon and primary dendrite, if to the same substance, must be opposite in character, or they must be reacting to different substances. The absence of definite orientation of the dendrites of some neurons in earlier stages, and its appearance later, and the sudden changes in direction of growth of both axon and dendrites make it necessary to assume that the reaction of the outgrowths changes, or that the concentration gradients of the substances change their direction, or that new substances appear with concentration gradients in different directions from those of the original substances. Again the establishment of functional connections between different neurons and between nerves and muscles and other peripheral organs necessitates various other assumptions. While directed response to chemical factors may play some part in neuron development, any attempt to interpret the process entirely in terms of such response is a pure speculation with many difficulties in the way.

It may perhaps appear at first glance that the situation is not much better as regards the interpretation in terms of electrical response, and so far as definite experimental data on the neuron itself are concerned, this is the case. Certain facts are at hand, however, which seem to me to be highly significant in this connection. In the first place, the existence of the axial physiological gradients and of gradients in electrical potential as one of their characteristics has been demonstrated beyond question, and we find a very definite 
relation between the directions of growth of the axons which arise in the earlier stages of development and these gradients. Second, from what we know concerning other developmental processes, there is good reason to believe that the establishment of regions of rapid growth in the nervous system, as well as in other organs, involves a change in electrical potential in those regions. Admitting this, it is evident that in the course of development there must be changes, both in the intensity of potential and in the direction of the potential gradients. Third, it is a familiar fact that conduction of the nervous impulse is accompanied by marked change in electrical potential, and as soon as any neuron, neuron group, or axon, or axon bundle becomes functionally active in this way, changes in the potential gradients in its vicinity must appear. In short, the existence of gradients in electric potential with the resulting electric currents and the changes in direction and intensity of these gradients during development and in relation to function must be a factor in determining the physiological condition of the cells affected, and so constitute a real basis of fact for attacking, at least tentatively, the problem of neuron development. On the other hand, it is not demonstrated, though many facts make it highly probable, that the neuroblast or the neuron is capable of response in any way to such electrical gradients, or, more strictly speaking, to the electrical currents which arise in connection with them. Moreover, the difficulties which arise in the matter of the opposite orientation of axon and dendrites in certain neurons and the absence of orientation in some or all dendrites in others, remain. But in spite of the lack of expe:- 
mental data concerning the neuron itself and in spite of the difficulties, it seems worth while to point out certain of the possibilites along this line of interpretation. ${ }^{x}$ First, however, it is necessary to examine a hypothesis, recently advanced by Kappers, of neuron polarity and growth orientation in terms of response to electrical factors.

KAPPERS' THEORY OF THE ORIGIN OF NEURON PATTERN

In a series of papers Kappers ${ }^{2}$ has described certain phylogenetic and ontogenetic processes of shifting or migration of neurons in the nervous system, which he has called "neurobiotaxis." Neurobiotaxis consists, according to Kappers (I9I 7 ) in the following phenomena: "The growth of the chief dendrite and eventually the displacement of the cell-body itself takes place in the direction whence the majority of stimuli proceed to the cell. Only between correlated centers does this outgrowth or shifting take place." In the growth of the axon also synchronic or successive stimulation plays a part. In attempting to account for the apparently directed outgrowth of dendrites and shifting of the cell body as well as for the localization and directed outgrowth of the axon Kappers has advanced the hypothesis that the electrical field in which a neuron develops is the factor which determines its morphological polarity as regards axon and dendrite. He interprets the growth response of the neuron in the electrical field as a galvanotaxis, the axon originating on the side of the neuroblast toward the positive pole or anode of the field and growing

I See footnote on p. 205 below.

${ }^{2}$ See Kappers, I9I 7, and references there given; also Bok, I9I5. 
toward the anode, the dendrites arising on the side toward the negative pole or cathode and growing toward the cathode of this field. Kappers accounts for the presence of the electrical field by the fact that a region of excitation becomes electronegative to its surroundings, and assumes that the electrical field to which the neuroblast reacts results from the stimulation of a neuron or group of neurons or axons in the vicinity of the neuroblast in question. The neuroblast itself he regards as surrounded by a fluid medium, the lymph, containing electrolytes and therefore conducting the current. According to the hypothesis, the neuroblast reacts to this electric current by localization and growth of axon and chief dendrite at opposite poles of the cell and later by shifting of the cell body toward the negative pole or cathode. The growth of the axon is then "stimulifugal," that of the dendrite "stimuli-petal."

As various authors have shown, the usual galvanotactic response of living organisms is toward the cathode, though this reaction may be reversed in many cases by changes in the surrounding medium, such as increase in concentration of electrolytes. If, however, the polarity of the neuron is a galvanotactic response of the sort Kappers postulates, it shows both positive cathodotaxis and positive anodotaxis. In order to account for this opposite reaction of axon and dendrites and for various other facts, such as the usual condition with one axon and several or many dendrites, the appearance of the axon earlier than the dendrites, etc., Kappers is forced to make a number of special assumptions, all of which have a physicochemical basis, but which seem to me greatly to complicate the hypothesis and limit its 
applicability. For example, the localization of the axon outgrowth is regarded as corresponding to the "anodic extension" observed by Verworn and others in Protozoa and certain other forms exposed to an electric current. Assuming that this anodic extension possesses a greater permeability for negative than for positive ions and referring to the evidence indicating that the medium about the neuroblast contains a considerable amount of potassium salts, Kappers maintains that the anodic extension will take up more negative ions than other parts of the cell and also more negative than positive ions and so will become electronegative and therefore positively anodotactic, i.e., it will be attracted by the anode and grow toward it.

The localization and outgrowth of the chief dendrites toward the cathode are regarded by Kappers as representing the normal or usual cathodotaxis. The dendrites grow out only after the cell contains Nissl granules, which are also present in the dendrites. These granules are supposedly of nuclear origin and therefore acid and electropositive, according to Kappers. He also suggests that the cataphoresis of albumens may play a part in the localization and direction of the outgrowths. Alkaline albumen particles move toward the anode and acid albumens toward the cathode of a current passing through the medium in which they are suspended. A more extended consideration of these and other special assumptions which Kappers makes to account for the facts is impossible and, I believe, unnecessary here, for I shall endeavor to show that they are not essential to an interpretation of the developmental processes concerned in terms of reaction to an electric current. 
Kappers' hypothesis may be illustrated by the accompanying diagram (Fig. 57). In this diagram $a$ represents the source of stimulation which Kappers postulates and which is electronegative externally; $d, a x$ is a neuroblast surrounded by a fluid medium containing electrolytes. The current produced by $a$ has its negative pole on the surface of $a$ and its positive pole in some more or less distant region, indicated by the sign + . The return current must pass in the opposite direction through body tissues. The neuroblast exposed
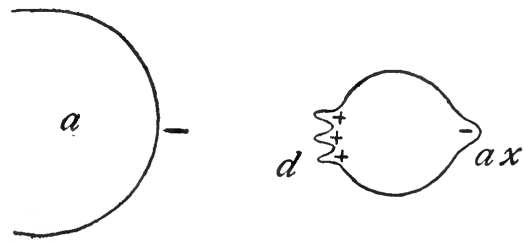

Fig. 57.-Diagram illustrating Kappers' conception of the action of the bioelectric current on the neuroblast. For explanation see text.

to the current arising from $a$ develops an anodic extension $a x$, which, because of its greater permeability to anions, becomes electronegative and gives rise to the axon growing toward the positive pole because of the attraction between it and this pole. Later the opposite side of the cell gives rise to the dendritic outgrowth $d$, which contains the supposedly acid and electropositive Nissl granules and is therefore attracted by the cathode and grows toward it.

While this ingenious hypothesis of Kappers' is of great interest as an attempt to interpret the polarity and external morphology of the neuron in terms of a reaction to an electric current, it seems to me to present certain 
difficulties, some of which may be briefly pointed out. In the first place, this hypothesis does not account for the outgrowth and orientation of axons and dendrites of the neuroblasts which first undergo differentiation in the nervous system, for it assumes the presence of functional neurons as the source of the current. Second, Kappers assumes that the neuroblast is more or less completely suspended in a fluid medium, consisting of lymph, containing electrolytes, while, as a matter of fact, it is in its earlier stages part of a very compact tissue, which may be more or less syncytial in character. At any rate, the cells are apparently in close contact, and tissue continuity exists to a high degree. Later in development of course the neurons do become more or less separated by the substance of the neuroglia, by lymph spaces, and probably by colloid gels, but differentiation begins long before these changes have occurred. Third, the hypothesis does not take into account in any way the metabolic activity concerned in the localization and outgrowth of axon and dendrites, but this is conceived as almost entirely a matter of electrical attraction between bodies of opposite sign. Actually, however, these processes involve localized metabolic activity, including oxidation and synthesis of new protoplasmic substance. Moreover, if positive cathodotaxis, such as Kappers assumes to underlie the outgrowth of the dendrites, is associated with cathodic stimulation, as many facts indicate, we should expect the side of the neuroblast toward the cathode to be more active than the other, and the dendrites should arise before the axon, but the reverse is the case. Fourth, the "anodic extension" observed by Verworn in certain forms in the 
current is by no means certainly an excitation, as Verworn regarded it. As a matter of fact, if the current is long continued or sufficiently strong, death and disintegration begin in this anodic extension. Kappers' assumption of increased permeability to anions in the anodic extension also seems to involve difficulties. In the current the anions migrate toward the anode, and unless the concentration of electrolytes in the medium surrounding the neuroblast is very much higher than inside the cell, which cannot be the case, the anions must migrate out of the cell toward the anode instead of into it. Consequently the anodic extension ought, according to Kappers' assumptions, to become more electropositive internally and therefore to grow toward the cathode, instead of becoming more electronegative and growing toward the anode, as he maintains. While these and other difficulties make it impossible to accept Kappers' hypothesis as it stands, there can be no question concerning the significance of the facts to which he calls attention, and concerning the difficulties involved in any attempt to interpret them in any other terms than those of relations of some sort to electrical factors. 


\section{CHAPTER XI}

SUGGESTIONS CONCERNING THE ORIGIN AND DEVELOPMENT OF NEURON PATTERN

ELECTRICAL POLARIZATION AS A LOCALIZING AND ORIENTING FACTOR

Most of the special assumptions as well as most of the difficulties involved in Kappers' hypothesis can, I believe, be avoided by considering the reaction of the neuroblast, not simply as a matter of electrical attraction, but rather as a change in physiological condition, resulting primarily from the electrical polarization of the cell through the action of the current to which it is exposed. When an electric current passes through any conducting medium in which ions are free to move, negative ions (anions) migrate toward the positive pole or anode and positive ions (cations) toward the negative pole or cathode. From this migration of the ions electrical polarization results. If the current passes through a living cell, it is evident that ions of opposite sign must migrate in opposite directions in the cell, and if the cell possesses a membrane which is not completely permeable to ions, and cells in general do possess such membranes, the ions of opposite sign must accumulate to some extent at opposite poles of the cell inside the membrane, the positive ions on the side toward the cathode, the negative ions on the side toward the anode. The cell is then electrically polarized and this polarization must determine changes in its physiological condition 
and activity. As I pointed out in earlier chapters (pp. 70-82), a region of excitation or of rapid oxidation is electronegative externally with respect to unexcited or inactive regions. This means that it is internally electropositive with respect to such regions. Increase in internal electropositivity is then associated with increase in the fundamental physiological activity of protoplasm, though it may also be brought about in other ways as well. Moreover, various lines of evidence and particularly the data concerning electrical stimulation indicate that an increase in internal electropositivity of living cells, directly produced by electrical means, generally if not universally determines a condition of excitation or an increase in fundamental physiological activity. It may be that in any stimulation of living protoplasm the primary change is electrical, but whether that is the case or not it is evident that the electrical change is a factor in the change in physiological state. If we admit this, it follows that electrical polarization of a cell may determine changes in its rate of metabolism and in its physiological state in general. ${ }^{x}$ We may expect to find the region of increased positivity in such a cell showing increased physiological activity or excitation, the region of increased negativity showing decreased activity or depression. As Hyman (1918) has suggested, the phenomena of electrotonus in the nerve fiber apparently fall under this head. When a constant current is passed through a portion of a nerve the irritability of a

${ }^{I}$ If the membrane of the unstimulated cell is normally polarized with internal surface negative and external positive, as commonly assumed, an increase in positivity in any region really constitutes primarily a depolarization of that region and such depolarization is, according to R. S. Lillie (see pp. 72-73), an essential factor in stimulation. 
region about the cathode, the region in which the nerve becomes internally positive and externally negative, is increased (catelectrotonus), and if the current is strong enough the cathode gives rise to a nerve impulse when the circuit is closed. A region of the nerve about the anode, however, where the nerve becomes externally more positive and internally more negative, shows decreased irritability while the current is passing, but the anode may give rise to a nerve impulse when the circuit is broken, that is, the disappearance of the depression constitutes an excitation. Electrotonus is apparently merely a special case of the physiological effect of electrical polarization in highly specialized tissues.

Electrical conditions as factors in development and differentiation have received comparatively little attention of late years, though they are probably of considerable importance, particularly in the case of highly susceptible cells, such as the neuroblasts, which in early developmental stages are the most susceptible cells of the organism. Concerning the presence of differences in electrical potential and of electric currents there can be no doubt, for, as I have pointed out (p. 44), they are characteristic features of the physiological gradients from the earliest stages on, and their existence in connection with the specialized activities of later stages has been abundantly demonstrated. It is of course not necessary to assume that electrical factors are the only factors concerned in determining the morphological polarity, directions of outgrowths, and connections of the neurons. Various other factors may play a part, but it appears impossible to find any other than the 
electrical factor which is adequate as the primary factor in these processes. The point may be emphasized, however, that according to the conception advanced here the electrical factor is effective, not directly as electrical but only through the changes in the physiological state brought about by it and the localization of those changes in relation to the electric currents.

The direction of electrical polarization in a given cell depends on the position of the poles of the polarizing current, and it must be pointed out that with respect to any particular source of current two opposite possibilities exist, depending on the relation of the cell concerned to the source. The situation as regards bioelectric currents is not fundamentally different from that in the galvanic cell. We call one pole of the cell positive, the other negative, but it is a familiar fact that the pole which is negative when the two poles are connected by a conducting medium outside the cell is positive with respect to the other pole inside the cell. We commonly say that the current flows in opposite directions inside and outside the galvanic cell. Similarly in the case of bioelectric phenomena, if a particular region of an organism is electronegative with respect to another, when the two regions are connected by a conducting medium, e.g., a wire, outside the protoplasm, it must be electropositive internally, that is, through the protoplasm, with respect to the other region. Here, as in the galvanic cell, the directions of the current inside and outside the tissue are opposite. As regards the electric polarization of a cell by such a current, it is evident that two opposed possibilities exist, according as the cell lies in one or the other half of the circuit. 
The following diagrams will assist in making the point clear. Let $a b$ (Fig. 58 ) represent a mass of continuous, closely coherent tissue, the cells of which are
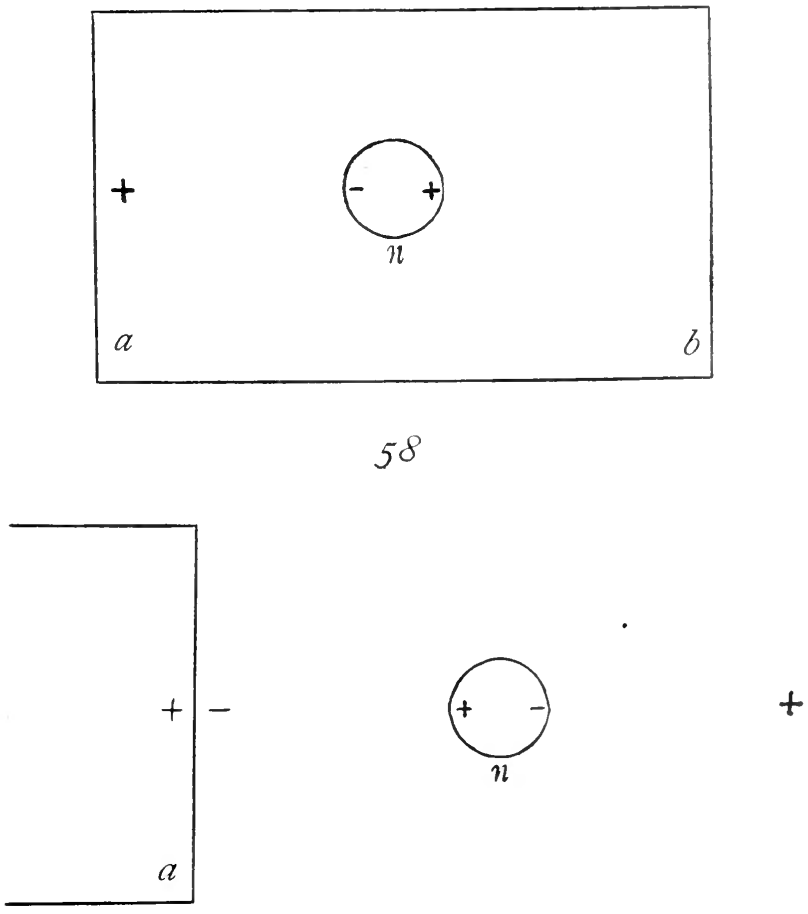

\section{9}

FIGS. 58, 59.-Diagrams illustrating electrical polarization of a cell by bioelectric currents: Fig. 58 , polarization of first type; Fig. 59, polarization of second type; further explanation in text.

in contact, such, for example, as the embryonic neural tube. In consequence of differences in physiological condition the region $a$ is electropositive internally to the region $b$ and a current passes through the tissue between 
the two. Positive ions will then migrate toward $b$, negative ions toward $a$. In a given cell, $n$, of the mass this migration will occur if the current passes through it, and the ions will accumulate at two opposite regions of the cell, the side toward $b$ becoming more positive internally, the side toward $a$ more negative. For convenience in the following discussion this is called the first type of polarization.

We turn now to the other alternative, Figure 59, in which the cell $n$ is not in direct tissue-continuity with the source of current $a$ but suspended in a conducting medium. The cell or tissue $a$ is electropositive internally, because of its physiological condition and is therefore electronegative externally, i.e., with respect to the medium in which the cell $n$ lies. Consequently in this medium positive ions will migrate toward the cathode on the surface of $a$, negative ions in the opposite direction. The cell $n$ will therefore undergo electrical polarization with the positive pole toward $a$, the negative in the opposite direction. This may be called for our purposes the second type of polarization. Comparing the two diagrams, Figures 58 and 59 , it is evident at once that although the relations as regards space and direction between the region of high positivity $a$ and the cell $n$ are the same in both cases, the polarization of $n$ is opposite. If the region of increased positivity in the polarized cell becomes more active physiologically and growth occurs in it in consequence of the polarization, the outgrowth in the first case (Fig. $5^{8}$ ) will be away from $a$ and toward $b$, and in the second case (Fig. 59) toward $a$. In the following sections a few suggestions are advanced concerning the occurrence of 
these two types of electrical polarization in the cells of the developing nervous system and their rôle in the differentiation, direction of growth, and connections of the neurons.

\section{AXON DEVELOPMENT}

In the localization and development of at least the earlier axons in the vertebrate central nervous system the conditions represented in Figure 58 seem to be more or less exactly realized. The embryonic neural tube constitutes a compact tissue and is the region of highest internal positivity in the embryo. Moreover, the region of highest internal positivity in this tissue is probably primarily that adjoining the original external surface (see p. 26), which becomes the surface of the central canal after closure of the tube (see Figs. I7, 62, 63). Secondarily, the edges of the neural folds which become the dorsal regions of the neural tube are, to judge from their susceptibility, the most active regions of the tube preceding the closure and therefore must represent regions of high positivity internally, as compared to more ventral regions. We may expect then to find that in the transverse section of the neural tube the electropositive regions of the neuroblasts, as determined by electrical polarization, will appear primarily on the sides of the cells away from the central canal and from the dorsal region, or in the resultant of these two gradients. Assuming that the region of highest positivity in each cell becomes the region of most rapid growth, extensions or outgrowths of the cells will appear on the sides of the cells away from the central canal, or the dorsal region, or in the resultant of these two directions and will grow laterally, or ventrally, or ventrolaterally 


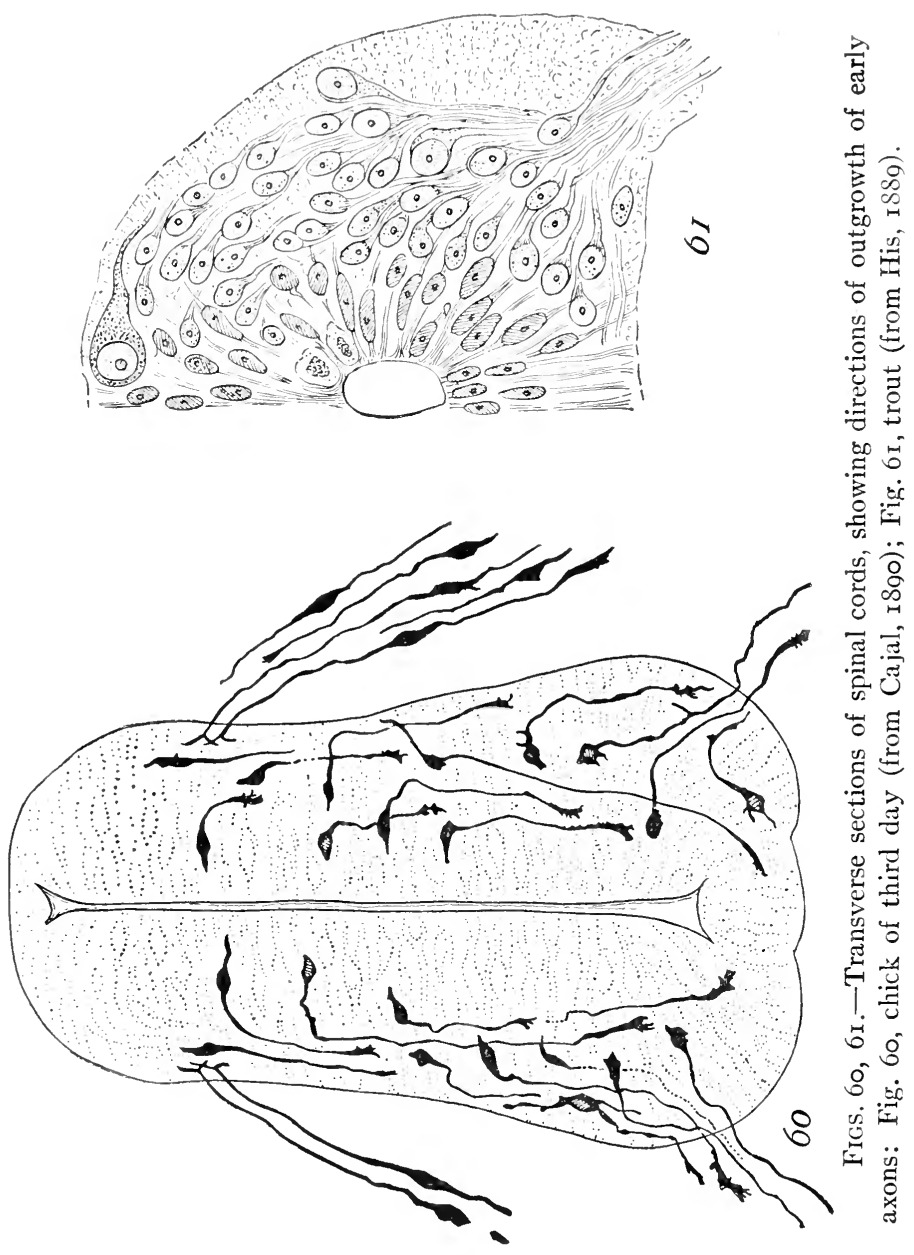


in the neural tube. Figures 60 and 6r, cross-sections of the cord of the chick and of the fish in early stages of neuron development, show very clearly the localization and directions of growth of the early axons and indicate their very definite relation to the central canal in the cells nearest it and to the dorsiventral direction in others. In short, the neuroblasts are apparently reacting to the physiological gradients, and the only factor in these gradients which we can at present conceive as determining polarity and direction of outgrowth is the electrical factor, acting through the polarization of the cells.

According to this interpretation, the region of origin of the axon in the neuroblast represents the high end of a physiological gradient, electrically determined in each cell, by the electric currents of its environment. The direction of axon growth may likewise be regarded as electrically determined but as a process of electrical and consequent physiological polarization rather than through a simple electrical attraction. Harrison (I908, I9ro) has shown that the growing tip of the axon is amoeboid and sends out pseudopodia, not merely in the direction of growth, but in various other directions (Fig. 56), and various axon tips in Figure 6o show similar conditions. The direction of pseudopod extension in the growing tip is evidently not determined by the electric gradient, but in general the pseudopodia which extend in the direction of the gradient will tend to persist, extend, and grow, because they, and particularly their tips, represent the regions of highest positivity.

An apparent migration of the cell body in the direction of axon outgrowth, i.e., toward the cathode of the current, is a feature of these early stages, at least in the 
development of the spinal cord. This is probably, like the axon outgrowth, a result of the electrical polarization of the cell, the side toward the cathode being more positive, more active physiologically, and growing more rapidly than, or even at the expense of, the other side. Apparent migrations resulting from such differences in relation to an axial gradient are of frequent occurrence in organisms. One of the most striking examples is found in the stolons of various hydroids. In the absence of food the tip of the stolon, which represents the high end of the axial gradient, grows at the expense of lower levels, and the stolon apparently creeps forward and may even become separated from the parent organism through the resorption of its more basal regions as nutrition for the growing tip. Such free stolons apparently wander about over the surface to which they are attached, always growing at the tip and undergoing resorption at the base, until they finally use up their own substance and disappear, or else, under certain conditions, give rise to a new hydranth and begin to feed. The apparent migrations of the neuroblasts and later of the neurons (p. 200) are perhaps due to such growth on the electropositive and resorption on the electronegative side of the cell.

The direction of growth of the axons is not necessarily altered by their emergence from the neural tube. Since they are protoplasmically continuous with the cell bodies in the tube, the electrical polarization of their tips may still remain the same. Consequently the general direction of growth of these axons will be down a resultant of the chief physiological gradients of the developing body. 
As the distance between the axon tip and the regions of high positivity in the chief body gradients increases, the positivity of the tip, and therefore its rate of growth, may decrease. In the connection of the axon with its peripheral organ other than electrical factors may perhaps be concerned. Chemical factors, for example, may play a part in some cases. It may be pointed out, however, that if the peripheral organ represents a region of relatively intense growth or functional activity, and therefore of high positivity internally, as the axon comes into its vicinity, it may bring about electrical polarization of the second type in the axon tip and so determine growth of the axon toward itself. It appears probable that the branching of peripheral nerves and the peripheral distribution and connections of axons with their end organs may be determined, in part at least, in this way through the developmental activity of particular organs, e.g., particular muscles, at the time when particular axon tips grow into their electrical field.

The growth inward of the axons of peripheral receptors in the invertebrates and in the olfactory primodium of the vertebrates is apparently a reaction of the first type (Fig. 58), like that of the axons in the neural tube, that is, it represents a growth directed by electrical polarization away from the region of high positivity just beneath the surface of the body. As the axons of peripheral receptors approach the central nervous system a polarization of the second type may arise with respect to the negative surface of the central nervous system and so determine their growth toward it. Inside the central nervous system the factors 
determining their distribution must be similar to those acting in the case of other axons. In many cases, however, the direction of growth of the axons of peripheral receptors is altered almost immediately after their origin by the presence of axial gradients, which are more effective than the surface-interior gradients in determining polarization and directed response. Even in the slightly differentiated sensory cells of the actinian tentacle the outgrowth apparently reacts almost immediately after its origin to the axial gradient of the tentacle and grows in most cases down this gradient (Fig. I8).

Axons may apparently grow up as well as down a general body gradient. According to the polarization hypothesis, such growth may result from polarization of the first type with respect to a region of high positivity in continuity with the axon, which gives rise to a current in the opposite direction from that of the general gradient and sufficiently strong to overcompensate it. In such a case the axon is in reality not growing up a gradient but down the local gradient to which it is reacting. Undoubtedly in the course of development of the central nervous system such local gradients frequently arise. For example, the approach of an axon or a group of axons to other cells less advanced in development or the localized activity associated with the development of a body segment may determine such polarization and direction of growth. An axon growing in a local gradient against a general axial gradient can of course grow only so far as its polarization is maintained by the local gradient. Such axons are therefore likely to be short, while those growing down the general gradients are much longer. A very 
interesting case in the development of the Amphibia is considered in another chapter (pp. 25I-57).

The aggregation of growing axons into nerve trunks is apparently also readily accounted for in terms of electrical polarization and growth. If the axon is internally positive, either on account of growth activity or later because of the passage of nervous impulses, it is negative externally and must therefore increase the positivity in that region of a later outgrowth which is toward its surface (second type of polarization). Consequently the later axons will tend to grow along the surface of those which have arisen earlier, except where other regions of organs give rise to a sufficiently strong electrical field to alter these relations.

In the origin of the branches known as collaterals from an axon local electrical fields more or less at right angles to the axon direction may play a part in localizing regions of high positivity and growth at various points along the axon and so determining the occurrence of budding. The axiate pattern of the axon suggests, however, that internal physiological factors may also play a part in this branching. The growing axon unquestionably represents, for the time being, a physiological axis with the most active region at its tip, and some degree of dominance and subordination probably exists between the tip and other regions within a certain distance from it. I have repeatedly called attention to the occurrence of physiological isolation in relation to such gradients and to its significance for the processes of budding and branching in multiaxial plants and animals (see chap. v). The axon with its branches bears a close resemblance to the growth forms of various 
plants and colonial animals with a main axis and lateral branches, and since it has been shown that physiological isolation is the primary factor in the development of such growth forms, quite independently of the specific constitution of the protoplasm, it is very probably concerned in the origin of branches from the axon. As the length of the axon increases, the effect of the tip on the more basal regions must decrease, until these regions are more or less physiologically isolated from it. Under these conditions, and perhaps also in relation to local electrical fields about the axon, budding may occur. Physiological isolation results not only through increase in distance from the dominant region, but also through decrease in activity of this region. In many central neurons growth of the axon ceases a short distance from the cell body and collaterals. In such neurons the appearance of the collaterals is probably associated with the cessation of growth in the main axon. Moreover, conditions such as this within the axon may render it more susceptible to the local electrical fields.

Kappers has called attention to the fact that the direction of growth of collaterals is usually at first more or less perpendicular to the axon from which they arise and accounts for this fact in terms of his hypothesis. From my point of view this direction of growth may be accounted for as follows: as soon as the growing tip of a collateral is established, its most positive region will be determined farthest away from the axon from which it arises, since this is more or less positive internally, either because growth is still going on, or because of the passage of impulses. Consequently the collateral will tend to grow at right angles to the axon for a certain 
distance, or until it enters some other electrical field capable of altering its direction.

The development of a terminal arborization on an axon may result in some cases from its approach toward an externally negative region of large size. Under such conditions the growing tip will tend to spread out and give rise to various outgrowths, all growing more or less toward the negative surface. Again, arborization may perhaps result from the cessation of the action of the previously directing field, so that growth becomes indefinite in direction or responds to - various local conditions, perhaps determined by adjoining neurons.

If these suggestions are correct, the axon behaves during its growth period in many respects like other physiological axes, and its origin, like that of other axes, is determined by a quantitative differential in the action of a factor external to it. The appearance or nonappearance of medullation on the axon is of course a matter of differentiation and therefore in part determined by the hereditary constitution of the protoplasm, though within the same individual differences in rate of metabolism in the developing axon are perhaps concerned in determining whether medullation shall appear or not.

\section{DENDRITE DEVELOPMENT}

The problem of the factors concerned in the origin and development of the dendrites appears much less simple than the problem of the axon, but the facts afford a basis for certain suggestions. In general the dendrites appear much later than the axon, they are evidently less highly specialized structures, their growth 
is usually less rapid, in some neurons they arise from all parts of the cell surface and some of them may show definitely directed growth, while others belonging to the same neuron grow in various directions, and in many cases only certain dendrites persist, others undergoing resorption in the course of development. Moreover, the dendrites undoubtedly are of great importance as nutritive organs of the neuron, as well as organs of conduction.

As regards the factors concerned in dendrite development, it appears necessary to recognize two distinct aspects or phases of the developmental process, the origin and localization of the dendrites on the cell body and the later orientation and definitely directed growth of some or all of them. In connection with dendrite origin two possible factors suggest themselves, viz., physiological isolation and the nutritive relations of the cell with its environment. Physiological isolation may lead to budding on the cell body as well as on the axon. The fact that the dendrites usually arise only when the axon tip has attained a considerable distance from the cell body, or has ceased to grow, favors this possiblity. From this viewpoint the origin of the dendrites is in some degree similar to the origin of the basal stolons in hydroids and perhaps of the root system in plants. But it is also possible that nutritive conditions in the cell body play a part in determining these outgrowths as they apparently do in root formation.

As regards the orientation and definitely directed growth of dendrites, we must apparently look to the electrical factor. By the time the dendrites arise, the developing neurons have become more or less separated 
from each other by the development of neuroglia and intercellular colloidal substance containing water and salts. The dendrites which show definite orientation grow toward certain axons or axon tracts of other neurons and establish synaptic contacts with them. Assuming that these tracts are regions of high developmental or functional nervous activity, electrical conditions are those of the second type of polarization (Fig. 59) in which the cell $n$ and the electronegative surface $a$ are not in continuity, but separated by a conducting medium. Under these conditions the dendrites must grow toward the electronegative surface of the axon tract. It is quite unnecessary to assume, as Kappers has done, that the dendrites react differently to the electric current from the axon. The physiological effect of polarization is the same in both axon and dendrite, but the two outgrowths develop, so to speak, in the two different halves of the electrical circuit, in which the currents flow in opposite directions.

The apparently selective or specific relations between the axons of certain neurons and the dendrites of others may then be determined by the developmental or functional activity of the one and its effect on the direction of growth of the other. Different dendrites of a particular neuron may enter different electrical fields and so establish connections with different neurons, and it is conceivable that some dendrites may remain nutritive organs.

The dendrite is evidently a less highly specialized axis than the axon, and its frequent branching suggests a low degree of dominance during growth. It reacts chiefly with reference to local conditions rather than to 
the more general gradients, because at the time of its development the simpler general gradients of earlier stages have undergone complication in various ways.

\section{THE NEURONS OF THE SPINAL GANGLIA}

The neurons of the spinal ganglia of the vertebrates present certain special problems. When the neural tube arises by the development of the neural folds and the borders of the neural plate (Fig. 62) and the meeting and fusion of these folds (Fig. 63), some of the cells of the dorsal border of the folds, the neural crest, are not incorporated in the neural tube, but migrate ventrally on each side of it (Fig. 64), forming, according to Johnston (I906, p. 50), segmental series of flaps attached to the dorsolateral surface of the cord and hanging down on its lateral surface. Later these flaps may separate entirely from the cord and form an aggregation of cells which constitutes the primordium of the spinal ganglion and lies between the cord and the mesoderm (Fig. 65).

In the process of differentiation each of these cells which becomes a neuron gives rise first to an outgrowth which extends dorsomedially and enters the cord, and somewhat later to a second outgrowth, which grows in the opposite direction (Fig. 60). Both of these outgrowths are structurally similar to the axons of other neurons and both may develop medullation. Functionally they constitute the afferent or sensory paths, and the direction of functional conduction in the peripheral outgrowth is toward the cell body instead of away from it, as in other axons. The question which of these outgrowths is axon and which dendrite, or whether both 

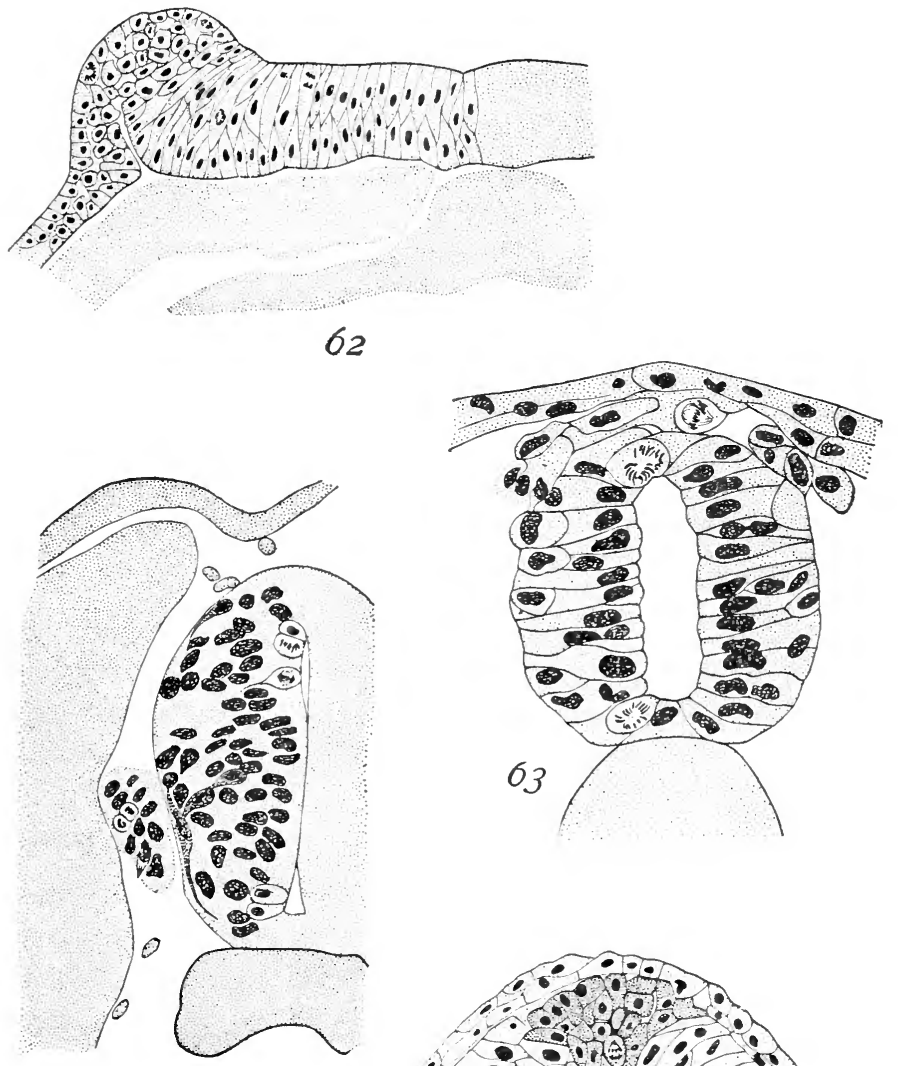

65

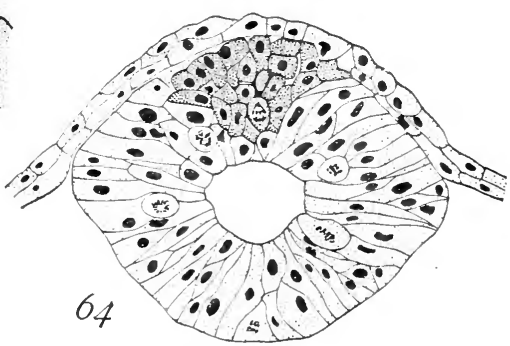

Figs. 62-65.-Developmental stages of neural tube and spinal ganglia in salamander, Amblystoma punctatum: Fig. 62, one side of neural plate, showing early stage of neural fold; Fig. 63, neural tube just after closure, cells of neural crest indicated by shading; Fig. 64, later stage, showing beginning migration of spinal ganglion cells; Fig. 65, still later stage; cells of neural crest have migrated lateroventrally to form spinal ganglion (from Johnston, I906). 
are axons, as they appear to be, is answered differently by different authorities. Some, taking into account the fact that the centrally directed outgrowth arises first and conducts away from the cell body, while the peripheral outgrowth appears later and conducts toward the cell body, regard the central outgrowth as axon, the peripheral as dendrite. When the peripheral process is separated from the cell body, chromatolysis occurs in the latter, as in other neurons after separation of the axon from the cell body, and this fact has led some to regard the peripheral process as the axon. Still others conclude from the structural characteristics that both processes are axons.

As regards the developmental factors concerned in this process of differentiation, three possibilities suggest themselves. The first is that the cells become electrically polarized while they are still continuous with the cord as the flaps mentioned above. Such polarization is of the first type (Fig. 58), and the region of high positivity determined by it must be ventrolateral. If this is the case, we should expect these cells to give rise first to axons growing ventrolaterally, but the ventrolateral migration of the cells may be a reaction to this polarization and may delay the axon outgrowth. As these cells lose their continuity with the cord, its electronegative external surface must tend to produce a second polarization in them, with the region of high positivity toward the negative surface, i.e., the dorsolateral regions of the cord. This is a polarization of the second type (Fig. 59), and it may also be a factor in retarding the outgrowth of the peripheral process because it tends to obliterate and reverse the primary polarization. If the effects of the 
first polarization are not immediately reversible, these cells may develop two growing regions at opposite poles and become strictly biaxial.

The second possibility is that the polarization giving rise to the dorsomedially directed outgrowth is the first effective polarization and that the peripheral outgrowth is determined only after the central process attains continuity with the cord. If this is the case, the migration of the cells before the outgrowths appear must be regarded as due to mechanical or other factors and not to polarization.

The third possibility is that both processes may represent reactions to polarization of the second type, the central process with respect to the most negative region of the cord, the peripheral with respect to the less strongly negative surfaces of peripheral parts, e.g., perhaps primarily the myomeres.

Which of these possibilities or whether any of them represent the actual course of events, it remains for the future to determine. They are not entirely mutually exclusive and each of them may be in part realized at some stage. In any case, however, it is evident that as soon as the centrally directed process attains continuity with the cord, the direction of growth of the peripheral process is determined in the same way and in the same general direction as that of the axons growing out from the ventrolateral regions of the cord (Fig. 58).

As regards the differentiation of both outgrowths as axons, various facts indicate that axon and dendrites do not represent reactions of different kind but rather of different degree, the more rapidly growing, more 
active, and probably more highly polarized region becoming an axon, the less active and less highly polarized, or perhaps not primarily polarized, a dendrite. Both outgrowths being highly polarized and active in this case, both develop as axons and the functional polarity of these cells, i.e., the direction in which they conduct, is determined, not by the fact that one outgrowth represents a dendrite, the other an axon, but by their relations to other parts. The reason for the occurrence in the cell body of chromatolysis following the section of the peripheral and not that of the central outgrowth, is not at present clear. It may be that the peripheral outgrowth makes greater demands on the cell body in the matter of nutrition and maintenance than the central, and therefore the effect on the cell following section of it is greater than that following section of the central outgrowth. The later change of the spinal ganglion neurons from the "bipolar" to the "monopolar" type by the approach and union of the bases of the two outgrowths is perhaps the result of "neurobiotaxis," which is considered below.

\section{KAPPERS' "NEUROBIOTAXIS" OF THE CELL BODY}

Kappers and others have shown that in many neurons and neuron groups the cell body undergoes an apparent migration in the direction of growth of the chief dendrites, i.e., toward the region with which the neuron or group is functionally correlated. So far as this migration is an ontogenetic, not a phylogenetic, process, it is doubtless determined by the factors which direct the growth of the dendrites and probably results from some degree of polarization of the cell body. On 
its more positive side, at this stage the side of the chief dendrites, the cell body probably adds to its substance by growth, while on the axon side it furnishes material for maintenance and further growth of the axon and therefore appears to migrate toward the region determining the polarization. Attention has already been called to the fact that in earlier stages, preceding or during axon origin, the cell body often appears to migrate in the direction of axon outgrowth (p. 187). The two reactions are apparently not different in kind, but represent rather the same reaction under different conditions.

\section{CERTAIN "MONOPOLAR" NEURONS}

Certain neurons, occurring particularly among the lower vertebrates in certain regions of the nervous system and as a characteristic feature of the invertebrate nervous system, possess a single main outgrowth or extension of the cell body, which for some distance remains dendritic in structure and usually gives rise to dendritic branches, but sooner or later decreases in diameter and becomes an axon (Figs. 5I, 52, 53). The primary outgrowth of such cells apparently corresponds, as regards its origin to the axon, although it is structurally dendritic and only gradually reaches the degree of specialization represented by axon structure. In fact, in some cells the outgrowth ends in dendritic branches and never gives rise to an axon. The occurrence of such neurons indicates clearly enough that dendrite structure and axon structure do not mean opposite reactions but rather different degrees of the same reaction. Either the degree of 
polarization and of the resulting growth activity is not sufficient to give rise at first to axon structure but gradually becomes so, or else these are cells which react to polarization more slowly than the ordinary type of neuron. Bethe has shown that in certain crustacean neurons of this general type (Fig. 52) the physical isolation of dendritic and axonic portions of the cell from the nucleate portions by section of the basal unbranched region of the outgrowths does not interfere with conduction from the dendrites to the axon. Evidently the nucleate portion of the cell has little or no direct relation to the nervous function but is concerned primarily with maintenance. This type of neuron represents then a gradual and partial specialization of the cell body, presumably in the region of high positivity determined by the polarization of the cell.

\section{THE CELLS OF THE NERVOUS RETICULUM}

These cells (Fig. 66), characteristic not only of the simplest nervous systems in the lower invertebrates but of certain organs in the higher animals, even the vertebrates, apparently have attained little or no definite developmental or morphological polarity. Their branching form and their apparent continuity with other cells may be due, at least in part, as Parker has suggested, to the fact that they represent the persistence of an embryonic syncytium. The absence of a definite structural polarity in these cells may be accounted for either by the indefiniteness and inconstancy of the physiological gradients in the organisms or organs concerned and the consequent indefiniteness and inconstancy of polarization or by the inability of the cells to 
react to such polarization as does occur. Both these factors may be concerned in many cases.

Even in the lower invertebrates, however, the reticular nervous structure without definite morphological polarity occurs chiefly in those portions of the nervous system which constitute the connecting links between the peripheral receptors and the muscles or other effectors. The peripheral receptors, which are in direct connection with the external surface of the body and therefore with the physiological factors concerned in surfaceinterior pattern, do possess a more or less definite polarity, and may give rise to an out-

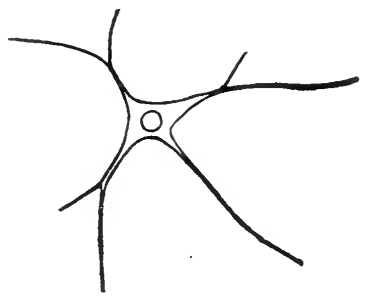

FIG. 66.-A cell of the nervous reticulum of a coelenterate; branches of such cells are apparently protoplasmically continuous with those of other cells of the reticulum. growth which shows definite growth direction with reference to an axial gradient. The receptor cells of the tentacles of certain sea anemones, for example, are not only elongated vertically to the surface, but give rise at their inner ends to nerve fibers which grow chiefly down the tentacle, i.e., down the gradient (Fig. I8, p. II2). A more or less definite polarity of this sort is a general characteristic of the peripheral receptors, even in the simplest forms in which we can distinguish definite nerve cells. Even the epithelial muscle cell of Hydra (Fig. 68, p. 238) which, as I have suggested in another chapter (p. 240), is probably to some extent a receptor and conductor as well as a muscle cell, shows a definite polarity with respect to the body surface. Evidently 
polarity may arise in the cells of these simple forms in relation to the surface-interior pattern and in some cases in relation to the axiate pattern. There can be little doubt that in these forms the surface-interior pattern is more definite and constant than the axiate pattern and that the physiological factors concerned in it are therefore more effective in determining, at least locally, a polarity and pattern in individual cells, than the general axiate pattern of the animal. If electrical polarization is concerned in determining such pattern, it is evidently of the first type, since there is tissuecontinuity, and the basal outgrowths of such cells correspond physiologically, as regards origin and direction of growth, to the axons of the more highly specialized neurons.

\section{CONCLUSION}

This and the preceding chapter constitute merely a brief consideration of some of the more important aspects of neuron development. The present chapter is nothing more than a tentative development of a conception of neuron polarity and external morphology on the basis of physiological factors concerned in organismic pattern. The factors themselves exist, and I have attempted to show that they afford a basis for interpretation of many facts of neuron development in relatively simple terms. That neuron pattern is and must be related physiologically to organismic pattern is, I think, beyond the possibility of doubt, and if the conception of organismic pattern developed in earlier chapters has any foundation in fact, it must afford a basis for attack on the neuron problem. This chapter is concerned with showing that it does afford such a basis. 
No one is more keenly aware than myself of the hypothetical character of the discussion and of the criticisms which may be brought against it. It is evident, however, that a consideration of the origin and development of the nervous system, such as has been attempted in this book, cannot stop short before the problem of neuron pattern and refuse to consider it. Neuron pattern is a feature, both of nervous and of organismic pattern. Moreover, the neuron itself develops an axiate pattern, and the resemblance of this to certain types of axiate organism, suggests the possibility of physiological resemblance. I have merely suggested an interpretation of neuron pattern in terms of organismic pattern. ${ }^{\mathrm{I}}$

${ }^{I}$ Since this and the preceding chapter were written, I have been informed through the kindness of Dr. R. G. Harrison, under whose direction the work was done, that Dr. Ingvar has succeeded in determining the direction of outgrowth of neuroblasts in tissue culture by means of electrical currents. A report of Ingvar's work is now in press and soon to appear in Proceedings of the Socicty for Experimental Biology and Medicine. This work represents, so far as I know, the first step in experimental determination of polarity in neuroblasts and, so far as it goes, constitutes an experimental basis for a hypothesis of electrical deternination, but much further work is of course necessary to determine whether or how far the particular hypothesis advanced in these chapters will account for the facts. 


\section{CHAPTER XII}

CERTAIN FEATURES OF TRANSMISSION AND CONDUCTION IN RELATION TO THE PHYSIOLOGICAL GRADIENTS

In chapter iv the part played by excitation and transmission in originating and establishing the physiological gradients was discussed. In chapters vi-xi it was shown that the physiological gradients afford a general physiological basis for the localization, the course of differentiation and the general plan of structure of the nervous system as a whole, as well as for tentative conclusions concerning the origin and development of neuron pattern. Since there can be no doubt concerning the close relation between nervous structure and nervous function, i.e., the conduction of impulses, and since the facts indicate that the physiological gradients originate in excitation and its protoplasmic transmission, there is every reason to believe that these gradients, once established, must play a part in the development of the more or less specialized forms of transmission, which are usually called conduction. The gradients provide, first of all, a mechanism for giving conduction a more or less definite, or at least a preferential, direction and thereby for determining in some degree the physiological relations to each other of various parts. The purpose of the present chapter is to call attention to certain facts in connection with the transmission or conduction of impulses in paths which do not show any distinguish- 
able nervous structure and to consider certain aspects of the problem of conduction in nervous tissue.

\section{THE DEVELOPMENT OF EXCITATION AND CONDUCTION}

It was pointed out in chapter iv that protoplasmic transmission in its most primitive form is probably little or nothing more than the gradient in physiological condition associated with the electrical gradient which results from a local excitation. With the development of protoplasmic excitability and conductivity, the transmission of what we call impulses, which consist of more or less definite waves of excitation, becomes increasingly possible. And, finally, the specialization of more or less definite paths affords the basis for a more definite conduction, as distinguished from the general protoplasmic transmission.

It is a familiar fact that the excitation of living protoplasm depends not merely upon the amount of change in action of an external factor, but also upon the rate of change. In fact the rate of change is, in most cases, if not always, more important than the amount. Similarly, the rate of change at which the excitatory process takes place at a given point is a factor of fundamental impor, tance in determining its effectiveness as a means of excitation of other points or regions, and so of transmission. A slow excitatory process is likely to be relatively ineffective, a rapid one much more effective. The development of excitability and conductivity in protoplasm very evidently consists in large measure of an increase in rate of the excitatory process and of the consequent increase in its effectiveness in exciting other points. Such development is a process not only of 
ontogeny but of evolution, and as such is of course associated with changes in the hereditary mechanism of excitation, however we may conceive such changes to be brought about.

The ascending phase, the front of the excitation wave or impulse, is itself essentially a physiological gradient which, instead of being stationary, advances over the protoplasm. The descending phase of the wave represents the fact that this gradient is readily and rapidly reversible, at least to a large extent. The more rapid the change from the unexcited condition to the completely excited condition, the steeper the gradient, and the steeper it is the more effective it becomes in inducing excitation at other points. With the increase in conductivity, i.e., the increase in the effectiveness of the excitation at any point in bringing about excitation at another point, the decrement becomes less and less steep, until in the vertebrate nerve fiber a condition is attained in which the "all-or-none law" holds, and conduction supposedly occurs without a decrement (see pp. 8I, 223). In such protoplasm a certain minimal degree of stimulation sets off, so to speak, the whole mechanism of excitation and a maximal excitation results automatically, just as a tremendous explosion may result from the reaction initiated by a minute spark. Concerning the actual physicochemical basis of these changes in the character of excitation in protoplasm we know little, but concerning the occurrence of the change there can be no doubt.

In this development of excitation rhythmicity frequently appears, that is, instead of the continuous existence of a state of excitation and its transmission or 
conduction definite waves or impulses appear, alternating with periods of rest. In one form or another rhythmicity in excitation is of very wide occurrence in organisms and ranges from such phenomena as the rhythmic impulses to ciliary movement to the heart beat. At this time it is impossible to do more than note the occurrence of rhythmicity.

While the development and evolution of the processes of excitation and conduction involve changes in the hereditary mechanism of protoplasm, there are certain features of the conduction pattern which are clearly related to the physiological gradients and certain others which may be interpreted in terms of such relation. Some of these relations have already been mentioned, particularly in chapters iv and $v$, but certain facts and questions remain to be considered.

\section{TRANSMISSION IN CERTAIN NON-NERVOUS OR NEUROID PATHS}

It is a familiar fact that in ciliated surfaces, whether composed of single cells or of many cells, forming an epithelium, the beat of successive cilia occurs in a definite sequence of such a character as to give the appearance of successive waves of bending and recovery of the cilia, passing over the surface. Such ciliated surfaces cover large parts or, in many cases, all of the external surfaces of many of the lower aquatic invertebrates and of early developmental-stages of numerous animals, both invertebrates and vertebrates, and are also found on many internal surfaces in various forms, ranging from the sponges to man. Each beat of a cilium is obviously due to an exciting impulse of some 
kind, and some sort of transmission must be concerned in the progress of the wave of beats over the surface. These ciliated surfaces occur in many animals and in stages of development where no distinguishable nervous structure exists, and even in cases in which a welldeveloped nervous system is present it has not been generally demonstrated that ciliary movement is under nervous control, although Pütter (1903) states that in certain animals such control does occur. Verworn (1890) maintained that transmission in such cases is purely mechanical, each cilium in its movement touching the next and so exciting it. Kraft (I890) and others have shown, however, that Verworn's view is incorrect, since transmission of the impulse may occur through a region in which the actual movement of the cilia has been completely inhibited by cold and after passing through such a region is still able to excite the cilia beyond. Brücke (I9r6) also found that when pieces of ciliated epithelium were cut out and reimplanted in the same spot in reversed position with respect to the direction of transmission, they showed a direction of transmission opposite to that in other regions of the epithelium. Such pieces evidently retain the original direction of transmission quite independently of any mechanical action of the cilia of adjoining regions. Mechanical transmission may perhaps occur in some cases of ciliary activity, but it is certainly not the fundamental factor in the passage of impulses over ciliated surfaces. The impulse is evidently an excitation of some kind and its transmission a protoplasmic process, but no definite conducting path is distinguishable. Parker (I905) has called transmission of this 
type "neuroid" transmission. While this term is undoubtedly useful, it should not be forgotten that such neuroid transmission represents merely a stage in the development and evolution of the excitationtransmission process and its morphological substratum.

Even though a definite, anatomical conducting path is not distinguishable, the direction of transmission of the impulses in ciliated surfaces is more or less definitely determined. Grützner (I882) and Kraft (I890) observed that the increased ciliary activity following direct mechanical stimulation of a local area of a ciliated epithelium is transmitted very largely in one direction from the stimulated area, the effect being observed as a band of increased activity, becoming somewhat broader with increase in distance from the stimulated area, but remaining definite in direction. Brücke's reimplantation experiment, referred to above, also shows that the direction of transmission is definite. As far as I am aware, no adequate physiological grounds for such definiteness of direction in the absence of a definite anatomical path have been discovered by earlier authors, but it is evident from what has been said in earlier chapters that the physiological gradients afford a basis for definitely directed transmission, even in the absence of localized and directed anatomical paths. We expect transmission to occur chiefly downward in such gradient, and, as a matter of fact, in those cases in which the direction of the physiological gradients is known this is very generally, if not always, the case as regards the usual or normal direction. In many ciliated surfaces the direction of transmission may be reversed by adequate stimulation of lower levels of the gradient, but 
such reversal means merely that a region of excitation arises in consequence of the stimulation and that the impulses initiated in it give rise to a temporary local gradient which overcompensates and reverses the general gradient for the time being and within a certain distance from the stimulated region, and so makes possible transmission in a direction opposite to the normal direction. The ctenophore plate row, discussed in the following section, affords beautiful examples of transmission and its reversal in relation to physiological gradients.

From the present viewpoint these cases of so-called neuroid transmission on ciliated surfaces appear, so far as the facts are at hand, to be merely cases of general cellular or protoplasmic transmission in relation to a general physiological gradient or to a temporary excitation gradient. The suggestion advanced by Parker (I905, p. 4I9) that neuroid transmission occurs irreversibly in one direction and that mechanical transmission is probably concerned in reversal is quite unnecessary. The directive factor appears beyond a doubt to be the physiological gradient and reversal to be associated with local or more or less extended reversal of this gradient by local excitation, or in some cases by developmental changes.

\section{TRANSMISSION IN THE ROW OF SWIMMING PLATES OF THE CTENOPHORE}

The body of the ctenophore possesses eight rows of motor organs, extending from a region near the aboral or apical end toward the oral end (Fig. 67). Each of the plates in a row is a flat, somewhat paddle-like 
structure made up of elements resembling large cilia placed side by side and adhering closely to each other. In movement each plate behaves like a single cilium. Under normal conditions an orally directed metachronism appears in the movements of the plates of each row, that is, the wave of beats begins at the apical end of the row with the beat of the first plate, the beat of the second plate follows slightly later and so on down the row. The movement of the plates and its progression down the row presents the appearance of a wave traveling in the oral direction. Such waves follow each other at widely different intervals, these be-

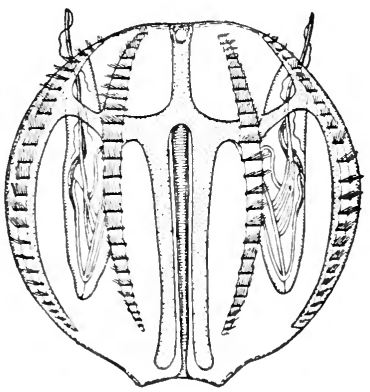

FIG. 67.- Side view of the body of the ctenophore, Pleurobrachia (from Hertwig, I905, after Chun). ing long, in some species several seconds, when the animals are completely undisturbed and quiescent, and much shorter, often only a small fraction of a second, when the animals are excited. Since the progress of the wave along the row is obviously the visible motor effect of a transmitted impulse of some sort, these plate rows constitute most interesting material for the experimental investigation of a relatively primitive type of transmission, and their behavior under various conditions has been repeatedly studied. Extended discussion of the literature of this subject is impossible at this time, but some of the earlier work has been considered in a recent paper (Child, I9I7C) and in an earlier study by Parker (I905). 
Before considering the behavior of the plate row, the question of the nature of the transmission requires some attention. Verworn (1890) believed it to be mechanical and others have supposed that a true nervous transmission is concerned, but Parker (I905) has concluded on the basis of experimental evidence that transmission in the plate row cannot be mechanical and that, since definite nervous structures have not been found, it must be neuroid. I agreed with this conclusion, but more recent observations of my own on several species raise the question whether true nervous transmission is not more intimately concerned in at least some portions of the path of the impulse than has been previously believed. About the apical sense organ are four tissue masses corresponding to the four quadrants of the body. In some ctenophores, e.g., Mnemiopsis, Bolina, a single ciliated groove passes orally from each of these four masses and at some distance from the apical end each groove divides into two, and each of the eight grooves thus formed connects with the apical end of a plate row. In other forms, e.g., Pleurobrachia, two ciliated grooves arise separately from each of the four masses and connect with the plate rows without division. When the animals are vitally stained with methylene blue a very definite differential staining occurs. The four tissue masses about the apical sense organ stain rapidly and deeply and appear as aggregations of cells. Each ciliated groove shows a perfectly definite, deeply stained strand, and the origin of this strand from the cell mass, its division into two in those forms in which it does divide and its connection with the plate row can be followed with the greatest 
ease. The four tissue masses are generally regarded as nervous in character and the very definite differential staining of these and of the strands arising from them make it highly probable that the strands are actually nerves. Each strand terminates in an expanded end in contact with the base of the first plate of each row. Methylene blue has been very widely used as a more or less specific stain intra vitam for nervous structures, and the very definite and sharply bounded differential staining of these structures in ctenophores leaves little doubt as regards their nervous nature. Moreover, in forms like Mnemiopsis, in which four strands arise from the apical masses and each strand divides into two and passes to two plate rows, the two plate rows thus "innervated" always show the same rhythm of beats, while in Pleurobrachia and other forms, in which two strands arise separately from each mass and pass without division to the two plate rows of a quadrant, these two rows often show an independent rhythm when the animal is more or less quiescent, but beat synchronously in stimulated animals. The cells of the plate row also stain with methylene blue earlier and more deeply than the general body surface, but no structures which can be identified as distinctly nervous appear in them.

In the light of all these facts I am inclined to believe that actual nervous connection exists between the plate row and the apical nervous tissue, and that the plate row, as regards its relation to this tissue, represents a motor end organ or effector, but one capable of initiating and transmitting impulses. The paths from the apical nervous tissue are, however, not the only paths of transmission, for, as various authors have noted, the plate 
row may be affected by direct stimulation of the mouth region or other parts of the body surface, even after the apical nervous tissue and the sense organ have been removed. Evidently also the plate row is not a pure effector, for it is able to initiate and transmit impulses. After section of the plate row at any level, transmission of the impulses from the apical region ceases at the level of section, but the portion of the row oral to the level of section begins, usually almost at once, or within a few moments, to beat with a rhythm of its own and the impulses are transmitted orally from the level of section. Moreover, even a single plate isolated by section from its neighbors may show rhythmic beats.

An investigation of the plate row in the genera, Mnemiopsis (Child, I9I7c), Bolina, Beroe, and Pleurobrachia (unpublished) by means of the susceptibility method shows that the row is a susceptibility gradient, its apical end being the most susceptible region. When individuals are exposed to the action of inhibiting agents, e.g., cyanide and various anesthetics, the apical end of the row is affected first, and at a certain stage the impulses from the apical organ appear at the apical end of the row but are transmitted only a short distance instead of the whole length of the row, as in normal animals. Under these conditions the lower levels of the row, being less susceptible, develop rhythmic impulses of their own. As I have pointed out (I9I7c), they are physiologically isolated. At first there may be only one such rhythm, originating in the region where the apical impulse disappears, but later, as the action of the agent progresses, several different rhythms may appear at different levels of the row, those of the more 
oral levels usually being at first more rapid than those of the more apical. In most individuals, particularly those of Pleurobrachia and Beroe, a second region of high susceptibility is present at the extreme oral end of the row, where new plates are developing as the animal grows. This region is less susceptible than the apical end, but more susceptible than the middle regions of the row. As regards its relation to the gradient of the row, this region corresponds to the secondary posterior growing region of many animals (see chap. viii). Since it is normally less completely under the control of the apical region, as various facts indicate, this oral region becomes physiologically isolated and shows independent rhythm at a very early stage of the action of inhibiting agents. Complete disappearance of transmitted impulses occurs first at the apical end and progresses orally along the row, except as regards the extreme oral region. Even after transmission is completely abolished in a particular region, independent movements of slight amplitude may continue for some time in single plates. Usually the apical and the extreme oral regions are dead and opaque white in consequence of colloid changes before inhibition in the least susceptible regions is complete. This sequence of events, which occurs in essentially the same way in all the rows at about the same rate, affords a beautiful example of the gradual obliteration of apical dominance and of the progressive physiological isolation of various levels of the gradient, as determined by the differences in susceptibility at different levels.

The plate row also shows certain very interesting features as regards reversal of the direction of transmission. Such reversal is most easily brought about in 
Pleurobrachia. In a normal individual of Pleurobrachia which has been undisturbed for a time and shows a slow rhythm, reversal is readily produced by direct mechanical stimulation of any level of the row. Such a stimulation usually gives rise to rhythmic impulses which are transmitted in both directions, and the rhythm of the impulse transmitted orally is almost always more rapid than that of the impulse transmitted apically. Evidently the region of stimulation is able to initiate impulses with greater frequency down the gradient than in the opposite direction. Moreover, the first impulses transmitted apically usually disappear within a short distance, often only two or three plates from the level of stimulation, but if the stimulation is continued they gradually extend their range and after a few seconds may be transmitted all the way to the apical end of the row. When impulses passing in opposite directions meet, the result differs in different cases. They may obliterate each other completely, so far as further motor effect is concerned, or one of them may obliterate the other and pass on, sometimes with a visibly lower rate of transmission than before, apparently because of its decreased intensity. In general the rate of transmission of the impulse passing toward the apical end is lower than that in the opposite direction, or becomes lower as the impulse travels.

In normal individuals of Pleurobrachia reversal of direction of transmission over the whole length of the row sometimes occurs apparently spontaneously for a short time, and it may usually be brought about by direct stimulation of the oral end of the row. In this case also the first impulses are transmitted only a short 
distance, but the range gradually increases with continued stimulation, until they arrive at the apical end. On cessation of the stimulation the reverse occurs, the normal apical impulses gradually extending their range as the oral impulses decrease theirs, until finally the normal direction is re-established. In the earlier stages of the action of inhibiting agents reversal of direction often occurs in Pleurobrachia without external stimulation, beginning at the oral end and often extending over the whole row. In such cases the greater degree of inhibition of the more susceptible apical regions has obliterated or reversed the original gradient to such a degree that transmission toward the apical end becomes the normal direction. Similar reversal of physiological gradients has been observed in many other cases. Some degree of reversal may be produced in Beroe by these methods, but usually only in the oral half of the row, and I have never seen the reversal extend to the apical end. In Mnemiopsis and Bolina reversal has been seen only at the extreme oral end and then only in the presence of inhibiting agents. Apparently the excitation waves are not high enough or not steep enough to travel up the gradient in these forms.

In Pleurobrachia and Beroe a difference in rate of transmission appears, not only with difference in direction, but with difference in degree of excitation of the animal. In a strongly stimulated individual the rhythm is much more rapid and the impulses travel more rapidly, in fact, almost twice as rapidly as in a quiescent animal.

That the ctenophore plate row represents a physiological gradient with a rise at its growing oral end is demonstrated not only by the susceptibility method 
but in various other ways. The behavior of the impulses with respect to the two directions not only constitutes additional evidence for the existence of the gradient, but is, I believe, inexplicable on any other basis. In this behavior we find in the first place dominance and subordination in the normal transmission, in which all levels are subordinate to the apical end, although each level is capable of initiating an impulse. Second, by decreasing experimentally apical dominance or by exciting subordinate levels to a sufficient degree, physiological isolation may be brought about, and, according to conditions, the isolated region either retains the original gradient or develops a new one, as in the case of reversed transmission. In fact, the plate row constitutes an example, almost diagrammatic in its clearness, of the dynamic relations of parts as determined by a physiological gradient. Whether the relations depend upon the presence of nervous structure or upon neuroid transmission is of minor importance from the physiological viewpoint. In any case the plate row exhibits the most various degrees of integration of the organismic type, and may, I believe, be regarded almost as a model of a physiological gradient.

\section{CERTAIN AXIATE ORGANS AS PHYSIOLOGICAL GRADIENTS}

It has already been shown that certain axiate organs behave in many respects much like the ctenophore plate row and probably further physiological analysis will show similar conditions in many other axiate organs. The vertebrate heart is a case in point. In its earlier embryological stages the heart is a tubular structure and Hyman has found that in both the fish and the 
chick (unpublished) the heart of the earlier stages shows a distinct susceptibility gradient, the end which is to become the sinus end being the high end of the gradient. This end becomes functionally the dominant region, the pacemaker, and the only conceivable basis for this dominance is the fact that it is originally the high end of the gradient. Its dominance depends essentially upon the fact that it is able to give rise to impulses with a more rapid rhythm than other levels under the same general conditions, and this ability is unquestionably associated with its position in the gradient. It makes little difference for this conception whether we accept the myogenic or the neurogenic theory of the heart beat.

If the sinus region is cooled or otherwise inhibited, the beat may be initiated by lower levels of the gradient, but the rhythm is slower. Any level of the heart is able to initiate a beat when sufficiently isolated from the sinus region, either by inhibition of the latter, by blocking the passage of the beat, or by sufficient stimulation of the level concerned. Even reversal in direction of transmission of the beat is possible to a greater or less degree under conditions which inhibit the sinus region and excite the arterial end, that is, which tend to reverse the gradient. In all these respects the heart as a conducting path and as a gradient resembles the plate row. The invertebrate heart differs more or less in form in different groups, but is in general tubular, and, though it has not yet been demonstrated to be a physiological gradient, it behaves in general like one.

The ascidian heart shows periodic reversal in the direction of transmission of the beat, the reversal occurring after a few or a considerable number of beats, 
according to species and conditions. The heart is tubular but evidently is not a fixed physiological gradient. I have already suggested elsewhere (I9I7c) that the reversal of the direction of beat is determined by fatigue of the temporarily dominant region with consequent decrease of its dominance. This decrease in dominance permits the other end of the tube, which is recovering or has recovered from fatigue, to initiate a beat and become dominant. The situation is practically identical with the spontaneous reversal which occurs in Pleurobrachia in the early stages of inhibition (p. 219) in consequence of the decrease in dominance of the more susceptible apical region and the physiological isolation of the oral end of the row.

Alvarez and his co-workers (p. 48) have demonstrated that the stomach, the small intestine, and the colon of mammals and of at least some other vertebrates represent physiological or metabolic gradients. Here, as in the other cases described, the relations of dominance and subordination exist and determine the normal direction of peristalsis, and physiological isolation and independent function of parts as well as reversal in direction of peristalsis may be brought about in essentially the same ways as in the plate row. Alvarez' evidence for the existence of the gradient rests on many different lines of experimental investigation and is now very complete. Moreover, he has pointed out that recognition of the presence of the gradient in the alimentary tract of the higher vertebrates and man is not only of theoretical interest but of great practical significance in medicine.

There is considerable evidence from its functional behavior that the ureter is also a physiological gradient. 
Portions of the circulatory system of various animals, which transmit active contraction waves in a particular direction, also probably arise as physiological gradients. In fact we shall probably find that all organs which are axiate as regards their special function as well as organisms and organs which are structurally axiate represent physiological gradients. The gradient is, in short, as I have attempted to show, the physiological basis and starting-point of the organismic type of relation, and structural and functional relations are merely different aspects of the general pattern.

THE “ALL-OR-NONE” PRINCIPLE IN NERVOUS CONDUCTION

The "all-or-none" principle or so-called law maintains that intensity or degree of excitation is independent of the strength or intensity of the stimulus for all stimuli above the threshold, the minimum stimulus which brings about reaction. In other words, in tissues which show the "all-or-none" type of reaction, all stimuli above the threshold produce maximal excitation. Lucas (I9I7) has concluded from his and Adrian's investigations that the nerve fiber, the axon, shows this type of reaction in the vertebrates.

Both Lucas and R. S. Lillie conclude further that if a conducting tissue, e.g., the nerve fiber, reacts according to the "all-or-none" principle, it must necessarily be capable of conducting to an indefinite distance without decrement an excitation once initiated. This conclusion is correct, provided the maximal excitation is the same in degree or intensity at all points of the path, in other words, if all points of the path are in the same 
physiological condition as regards their capacity for excitation. If, however, the conduction path is a predetermined gradient the maximal excitation may differ quantitatively at different levels of the gradient, that is, a gradient in the quantitative characteristics of maximal excitation may occur along the path. Moreover, we might expect to find a rise in threshold with progress down the gradient. Such a path, even though the reaction is strictly "all or none" at all levels, may conduct with a decrement. If it is long enough, a limit may even be reached, beyond which conduction cannot proceed because the stimulus resulting from maximal excitation at this point is so weak that it falls below the threshold for points beyond. The "all-or-none" type of reaction does not then exclude the possibility of conduction with a decrement and of a limited range or distance of conduction. In such a path, however, the steepness of the decrement and the position of the limit are determined by the path, not by the quantitative characteristics of the original stimulus.

As pointed out in chapter iv, the primitive forms of transmission do not show the "all-or-none" reaction, but quantitatively different stimuli determine quantitatively different reactions between certain limits. A slight stimulus may produce a slight response only in the region immediately about the point of stimulation, a stronger stimulus a stronger response, which is transmitted to a greater distance, and so on up to the maximum. Even in more or less specialized conduction paths like the ctenophore plate row such differences appear and are different in degree according to the direction of conduction in the gradient. Even in the 
nervous reticulum such differences have been observed in various cases. In the coelenterate tentacle, for example, impulses pass more readily and to greater distances basipetally than in the opposite direction. But the observations of Mayer (I906, I908, I916) and Harvey (I9I2) on conduction in the medusa, Cassiopea, indicate that in this form the nerve net may conduct both with and without a decrement. Slight stimuli may produce reactions which disappear within a short distance, but the conduction of the "trapped" excitation wares in circular strips of the medusa body may continue around the strip for days, or until it dies, without appreciable decrement.

It is evident from these facts that in Cassiopea some submaximal excitations are conducted with a decrement, while maximal excitations, or those above a certain quantitative level, are conducted without appreciable decrement, at least in the direction around the body. Apparently in such cases as this the weaker excitations do not set in action the whole of the excitatory mechanism, perhaps do not bring about certain chemical reactions or other changes, and the effects of such excitations as stimuli on following points of the path also give partial excitations weaker than the original. Maximal or sufficiently strong excitation, however, sets the whole mechanism in action and brings about the same result at following points of the path; consequently no decrement appears. Whether such differences in conduction are characteristic of the reticular type of nervous system is not yet known.

It is generally assumed that the axon of the higher animals conducts without a decrement, but it has 
already been pointed out that if the nerve fiber is a physiological gradient when the impulse passes, a decrement may occur, even though the reaction be "all or none." During its growth period the axon possesses a very active growing tip, and for the time being a gradient probably extends a greater or less distance from this tip. When the axon reaches its end organ the tip as a region of special activity disappears, and the gradient associated with the tip probably disappears soon after. Exactly such changes as these occur in many plant axes of limited growth. A gradient present during the growth period in relation to a growing tip may disappear completely after the tip ceases its activity at the end of the growth period, and in some cases new gradients may arise. In general the axon grows down a general or local gradient, and after its growth ceases it lies in the electrical field of the gradient which determined its growth, provided this gradient persists. It seems probable that under such conditions the different levels, particularly of the longer axons, must often be in different physiological condition. Moreover, the relation of the axon to the cell body, whether it be a matter of transportative or of transmissive correlation, may determine differences of condition at different levels of the axon.

The evidence, as far as it goes, indicates that these gradients do exist in some axons, at least. Tashiro (I9I4, I9I $5 a, b$ ) has found a gradient in $\mathrm{CO}_{2}$ production in the resting nerve, decreasing in the direction of functional conduction, and in certain of the nerves investigated by Tashiro I have found a susceptibility gradient in the same direction, as indicated by the structural 
death changes (Child, I9I4a). Moreover, Adrian's data on the frog's sciatic indicate that more peripheral levels of the nerve are slightly less susceptible to the action of anesthetics (Lucas, I9I7, chap. iv), though the author attributes the difference to defects in the apparatus. The frog's sciatic is unfavorable material for the detection of a gradient because the length of nerve available is not great. If a gradient is present in the axon it certainly cannot be steep, and in many cases it may be appreciable only in long nerves. As the matter stands at present, the experimental evidence indicates that gradients do exist, at least in some axons, and I have shown that the "all-or-none" type of reaction is not in the least incompatible with the existence of these gradients.

Even if we admit that physiological gradients are present in axons after the growth period is over, the limit of conduction is certainly not reached nor even approached in the higher animals within the lengths of axons attained in the body. Possibly in some of the elongated lower vertebrates and invertebrates the axon length may approach the limit, but on this point we have no data. If it exists then in the higher forms, the axon gradient is important, not as determining functional relations, but rather as a record, an effect of the physiological gradients of the body.

When the vertebrate nerve is cooled or partially anesthetized, a relatively steep decrement in conduction appears, as many investigators have shown. Apparently under these conditions the more susceptible parts of the excitatory mechanism have been put out of action, so that the excitation which occurs is only partial and 
its effect as a stimulus on following points produces an even weaker excitation. A relatively steep decrement therefore appears in the cooled or anesthetized region, but if this is not so long that the excitation becomes completely ineffective the impulse regains its normal characteristics when it emerges from the experimentally altered region.

These and various other characteristics of nervous conduction suggest that the evolution of the excitatory process has consisted in part in the modification of the primitive mechanism in the direction of high intensity, but such modification evidently also involves high susceptibility. Inhibiting agents and conditions first eliminate, reversibly or irreversibly, these highly susceptible features of the mechanism, but affect the more primitive factors more slowly. At certain stages of such action, therefore, highly specialized paths, such as the axon, appear to lose some of their specialization, and their behavior resembles that of more primitive paths.

\section{CONDUCTION IN THE NEURON AND THE PROBLEM OF THE SYNAPSE}

In its functional relations as a part of the nervous system the neuron conducts impulses from the tips of the dendrites to the tips of the axons, but if an axon is artificially stimulated at some point, the electrical change, the negative variation, which accompanies the passage of a nervous impulse, runs in both directions from the point of stimulation. If this negative variation is a characteristic feature of the nervous impulse, as there is every reason to believe, the axon is evidently capable of conducting impulses in both directions, but, so far as we know, the impulse which passes toward the 
cell body in an axon never affects another neuron. ${ }^{\mathrm{I}}$ Conceivably such an impulse may be unable for some reason to pass the cell body, or it may be unable to pass through the dendrite, or finally it may be unable to pass the synapse. Current views regard the synapse as chiefly responsible for this functional irreversibility of direction of conduction from one neuron to another. Moreover, blocking, delay and the phenomena of summation of impulses in the reflex arc are ordinarily attributed to the synapse. According to Lucas (I9I7) the junction between motor nerve and muscle is a region in which the nervous impulse apparently undergoes a decrement, and he suggests that conditions at this junction may throw some light on the problem of the synapse. To account for the delay and for summation, it is commonly assumed that the synapse differs in some way from other parts of the nervous system as regards the excitation process in it. Either it is not readily excitable or the intensity or height of the excitation wave in it is less than in other parts. Consequently it constitutes to a greater or less extent an obstacle in the way of the impulse, but continued or repeated excitation may alter its condition so that the impulse is able to pass. Passage, however, is apparently in one direction only, from axon to dendrite, and some further assumption is necessary to account for the irreversibility of direction. Membranes, more or less irreversible as regards direction with respect to passage of chemical substances, do occur in other organs, but the physicochemical basis of this characteristic is not known with

${ }^{x}$ If the peripheral processes of the spinal ganglion neuron are axons (see p. 196) these cells constitute an exception to this general rule. 
certainty. The synapse may be a membrane of this sort as regards excitation, but we know nothing of the mechanism of such irreversibility. Since this theory of the synapse is still in large measure based on assumption rather than on experimental data, it is perhaps allowable to point out the possibility of a different interpretation of some of the characteristics of the reflex arc, ordinarily attributed to the synapse.

The functional polarity of the neuron is evidently quite distinct from its developmental polarity, as it exists during the growth period. It is clear that a relation of functional dominance and subordination exists between neurons, and such a relation may depend in part upon the order in which the neurons of a given path attain the stage of functional conduction of impulses and in part upon the frequency or the quantitative characteristics of functional impulses in other neurons in their vicinity. The facts indicate that the neurons of the higher levels of a particular gradient develop more rapidly than those of lower levels, and it is also probable that the nervous impulse represents, at least at first, either a steeper or a higher wave of excitation in a neuron of higher than in one of lower levels of a gradient. If differences of this sort exist, they must constitute important factors in determining not only the morphological connections of different neurons but the functional relations between them and particularly such matters as "openness" or "resistence" of functional paths.

As regards the pecularities of inter-neuronic conduction which are usually attributed to the synapse, the possibility that certain characteristics of the dendrite 
as a conducting path and not merely the synapse itself, may be concerned deserve attention. The dendrite is much less highly specialized morphologically than the axon and is evidently concerned in nutrition and maintenance of the neuron as well as in conduction. We know practically nothing concerning conduction in the ordinary dendrite, but it is evident that the assumptions usually made concerning the synapse may be made, perhaps on even better grounds, concerning the dendrite. If the dendrite is less readily excitable than the axon, does not show the "all-or-none" type of reaction, and conducts with a steep decrement, it will serve as well as the synapse to account for the characteristics of the reflex arc commonly attributed to the synapse. Assuming for the sake of argument that the dendrite possesses these characteristics of the more primitive conduction path, an excitation conducted up the axon, through the cell body, and down the dendrite may be so slow or so weak or both, by the time it reaches the tip of the dendrite that it is unable to excite the axon tip with which the dendrite is in contact. The excitation wave in the axon, being steeper or higher, or both, than that in the dendrite and undergoing little or no decrement in its passage down the axon, is able to excite the dendrite or the cell body, with which the axon is in contact.

As regards delay and summation, it may require a number of impulses from the axon to bring the dendrite to a physiological condition in which it is able to initiate an excitation wave, high enough or intense enough to travel to and through the cell body to the axon. In the conduction of impulses toward the apical end of the ctenophore plate row following mechanical stimulation 
of a lower level, essentially this change occurs (p. 218). The first impulses travel only a short distance from the point of stimulation, but if the stimulation is continued they travel successively farther and farther, until after a time an impulse is produced which reaches the apical end of the row. In this case the continued stimulation and the passage of impulses upward doubtless affect both the condition of the path and the quantitative characteristics of the later impulses. This is obviously a sort of summation. 


\section{CHAPTER XIII}

THE REFLEX ARC IN RELATION TO THE GRADIENT THE PHYSIOLOGICAL CHARACTERISTICS OF REFLEX ARC AND EXCITATION GRADIENT

Students of the nervous system are agreed that the reflex is the unit reaction (Sherrington) or the physiological unit (Parker) upon which nervous function and integration are built up, but they are not in complete agreement as to what constitutes a reflex unit's simplest terms. According to Sherrington (I906), for example, a reflex arc consists fundamentally of receptor, conductor, and effector, while Herrick's conception (Herrick, I9I8) and Parker's (I9I9) also involves the presence of a central organ, the adjustor, and so of a central nervous system.

But wherever we may determine to draw the line between the true reflex and the simpler response it is evident that we have in the reflex arc, first, the initiation of excitation in the receptor by some external agent, second, the transmission of excitation over some path, the conductor, from the point of excitation, usually with more or less central complication, and third, the production by the transmitted excitation of some effect through the agency of a more or less specialized organ, the effector. In the higher animals even the simpler reflexes are rather highly adapted reactions in which the biologically purposive character is more or less evident, and the reflex arc in such cases represents a 
considerable degree of physiological integration, since it correlates two more or less different organs, the receptor and effector, or, more strictly speaking, subordinates one to the other. Even in the primitive receptorconductor-effector system of Vorticella within the limits of a single cell and without any visible nervous structure there is a considerable degree of integration and biological purpose.

Every neurologist and physiologist recognizes that between the highly adaptive reflex arcs of the vertebrate with their highly specialized receptors, conductors, and effectors, and the sensitivity and conductivity of living protoplasm in general some sort of relation must exist. The problem of the physiological origin of this adapted unit reaction, the reflex, is a part of the problem of the origin and evolution of the nervous system in its physiological aspects and its consideration is the first step toward any adequate conception of the nervous system as a functional system.

Viewed from its physiological side the reflex consists in initiation, transmission, and end-effect of excitation induced by some external agent. From this point of view the relationship is evident between the reflex arc and the protoplasmic excitation gradient, which is the first step in axiate integration. In the primitive protoplasmic gradient, receptor, conductor, and effector are not differentiated, but any part of the protoplasm or at least of the protoplasmic surface may perform any of these functions. The receptor is merely the point first or most intensely excited by an external stimulus, the conductor is any protoplasm which conducts or transmits the excitation, and the effector is any point excited 
by the transmitted excitation. In fact each point of the path is successively receptor, conductor, and effector, the effect being excitation, consisting of chemical reaction and other changes, the liberation of energy, and the excitation of a further point. As long as the protoplasm concerned remains undifferentiated, such an excitation gradient can never develop a characteristic feature of the true reflex, viz., the specialized function of the effector, which constitutes a part of the foundation for the adaptive character of the reflex, but the general physiological basis on which the development of the reflex may occur is clearly present in such a gradient. In short, according to this viewpoint the physiological basis which underlies and initiates orderly development and morphological and physiological unity and order in general is the same as the basis of nervous integration and of the most complicated nervous acts. The physiological axis and the reflex arc have a common origin in the protoplasmic excitation gradient.

For the sake of convenience and clarity it is necessary before proceeding farther to make certain definitions. An excitation gradient is the change resulting from irritation or excitation at some point and its protoplasmic transmission with a decrement (pp. 75-82). In such a gradient each level may be successively receptor, conductor, and effector. Through gradual changes in irritability and protoplasmic conditions at different levels an excitation gradient may become a more or less permanent axial gradient or physiological axis of a whole organism or of some part of it. As soon as local specialization and differentiation begin at different levels of a gradient it must be distinguished from a simple gradient 
because the specialization may alter the primary relations, e.g., an effector at a certain level, although subordinate to the receptor-conductor portion of the system, may show under excitation a more intense activity than levels of the gradient above it. An excitation system in which local specialization of receptor, conductor, and effector, or of some of them, has begun may then be distinguished physiologically from a simple gradient as an excitation arc. Morphologically as well as physiologically, of course, it may differ from the simple gradient in an indefinite number of ways. Except as regards the highly adaptive character and the central complications in the reflexes of higher animals, such an excitation arc is already similar physiologically to a reflex arc, though it is not necessarily associated with visibly differentiated nervous structure. With the evolution of the adaptive character, i.e., the preferential development, persistence, or low "functional resistance" of certain arcs which serve a biological purpose, the arc may be conceived, as Dewey (1893) suggests, as physiologically a circuit.

It is perhaps necessary to call attention to the distinction between organismic and protoplasmic pattern involved in what has been said concerning adaptation in the reflex arc. Even the simplest organism capable of responding in any way to the action of an external factor has unquestionably undergone adaptation as regards its protoplasmic pattern, but a physiological gradient in it may be a matter of direct response of this protoplasm to external factors. This gradient pattern itself is not adaptive in character, though the protoplasmic pattern on which it is based may be highly adaptive. A 
simple excitation gradient, arising in direct response to external excitation, is not as a whole an adaptive pattern.

The problem of the evolution of a true adaptive reflex from the protoplasmic excitation gradient is a problem of the specialization and adaptation of function and structure. The establishment of definite axial relations in the organism and so of definite relations to the external world affords a physiological basis for the localization and differentiation of definite receptors, conductors, and effectors, and with the beginning of the specialization of these as distinct organs, the reflex in its essential physiological features is present. The adaptive features of the reflex are chiefly in the central organ, the adjustor, and the relations of the particular arc to it. In this aspect the reflex arc becomes a product of evolution, and its development is a historical process associated with biological purpose. At present we are concerned rather with the discovery of the physiological basis of such adaptive evolution than with the evolution itself.

\section{HISTORICAL AND CRITICAL}

If the physiological basis of the reflex arc is an excitation gradient of the same sort as the gradients which underlie axiate order and integration, we should expect to find receptor, conductor, and effector associated with each other, not independent in their evolutionary history. The relation between receptor and conductor is self-evident and leaves little room for discussion, but as regards the relation of the effector to the rest of the arc the physiological facts are not so clear, and a wide difference of opinion has existed. 
Kleinenberg (I872), for example, held that the primitive condition with receptor, conductor, and effector within the limits of a single cell is found in the neuro-muscle cell of the Hydra ectoderm (Fig. 68). The outer exposed surface of the cell is the receptor, the elongated contractile base the effector, and the intermediate region the conductor. The effect of excitation in this unicellular arc is motor in character, a

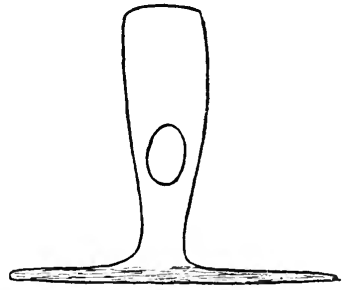

Fig.68.-An epithelio-muscle cell ("neuro-muscle cell") of Hydra, the contractile basal portion indicated by shading. simple contraction of the basal effector portion of the cell. According to this conception, receptor, conductor, and effector develop in functional relation, first within a single cell, and only later in the course of evolution become separated into different cells. The brothers Hertwig (I878) agree with Kleinenberg as regards the simultaneous development of nerve and muscle, but their studies on the medusae lead them to believe that receptor, conductor, and effector arise as separate cells (Fig. 69). Claus (1878) and Chun (I880) maintained that the nervous system arises independently of muscles and only secondarily becomes connected with them, while Samassa (1892) and Schaeppi (1904) hold that a nervous system without effectors is difficult to conceive. More

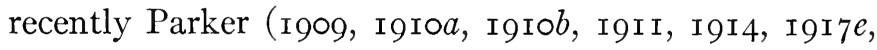
I9I9) concludes from his study of sponges that muscles appear first as independent effectors and in connection with them the first true nervous organs in the form of receptors, sensory cells, develop, a condition very similar 
to that in the coelenterates, while the central organ, the adjustor, is of still later origin. With the exception of Kleinenberg's conception of the neuro-muscle cell, these hypotheses and suggestions are concerned more with the origin of the nervous system as a morphological structure than as a physiological entity. A brief consideration will show that from the physiological point of view these different views appear rather as special cases of a general physiological process than as mutually exclusive conceptions.

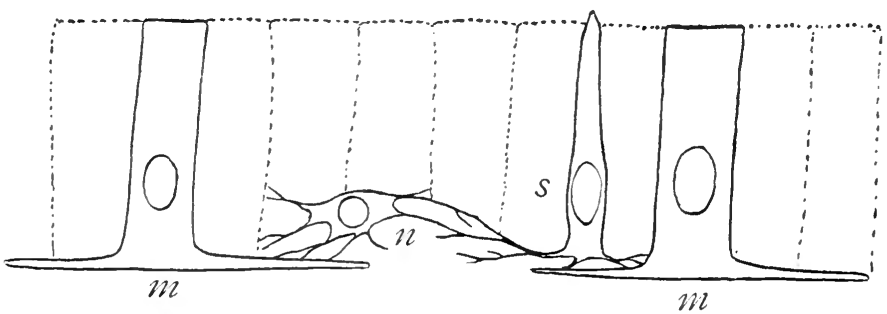

Fig. 69.-Diagram of simple multicellular arcs: $s$, sensory cell (receptor); $n$, nerve cell (conductor); $m, m$, muscle cells (effectors).

If the reflex arc develops from the protoplasmic excitation gradient, the development and evolution of the nervous system as a morphological structure must follow in general the lines of the chief or the most persistent excitation gradients established in the protoplasm of a particular species. According to the conception of physiological axiation developed in the earlier chapters, an excitation gradient may arise in primitive protoplasm at any point of the surface where excitation occurs, and any particular gradient may persist only momentarily or for a short time, as is apparently the case in Amoeba (Hyman, I9r7). Under 
such conditions the development of a definite, persistent receptor-conductor-effector structure is of course impossible. But where the gradients persist, whether from the surface inward or as axial gradients of the body as a whole, they afford a physiological basis for the development of a structural nervous system. In a multicellular organism, such as Hydra, where the body cells form coherent epithelial layers and only certain portions of the cell surface are directly exposed to the extra-organic environment the conditions exist for the development of an excitation gradient and a physiological axis in each ectoderm cell with the exposed surface as receptor, and this pattern may coexist with a general axiate pattern of the whole body. The visible differentiation of an effector region, e.g., a contractile portion, in such a cell may occur before anything recognizable as nervous structure is visible, for reception and transmission of excitation apparently do not bring about modification of protoplasmic structure as rapidly or as extensively as the development of contractility. In short, the neuromuscle cell of Kleinenberg is exactly the sort of system we might expect to find in a cell layer with definite surface relations to the exterior. It represents simply one possibility in the origin of a receptor-conductoreffector system, and the grounds for doubting its existence are morphological rather than physiological.

Parker's conclusion that the myocytes of sponges are independent effectors is doubtless morphologically correct, but physiologically either some portions of these myocytes themselves function as receptors and conductors or else other cells perform these functions. The presence or absence of anything recognizable as 
nerve cells or fibers is physiologically of secondary importance. As a physiological entity the receptorconductor-effector system is present in the sponges, either in single cells or in cell series, and if the myocytes react directly, without relation to other cells, they themselves must be physiologically more or less like neuro-muscle cells. Physiologically speaking, a pure effector is impossible, for even the most highly specialized effector must be capable of receiving stimuli from without. As a matter of fact every part of the reflex arc like the primitive excitation gradient functions as receptor, conductor, and effector, at least in one direction. Nevertheless the different regions of the arc are in the broad sense predominantly receptors, conductors, and effectors, as their differentiation indicates. In this sense it is undoubtedly true, as Parker maintains, that effectors appear, i.e., become morphologically distinguishable earlier than nervous structures. The condition in the medusae, as described by the Hertwigs, in which receptor, conductor, and effector are different cells, does not by any means exclude the possibility of the existence of the neuro-muscle cell, i.e., the system in unicellular form, even in the same individual. In Figure 69, for example, where the system as conceived by the Hertwigs is shown, it is certainly possible that the contractile basal portion of the cell $m$ may be activated not only by the receptor-conductor system represented by the other two cells, $s$ and $n$, but by excitation at its own receptor surface and transmission in its own protoplasm. Such a cell may be a neuromuscle cell and at the same time its contractile portion may be the effector of one or several other arcs. 
Moreover, the excitation arc may arise not only within a single cell or within a small group of adjacent cells, but also in relation to the axiation and integration of the body as a whole. In other words, not merely intracellular gradients and local multicellular gradients, but the general axial gradient or gradients of the whole body may constitute the basis of excitation arcs and so of nervous function and structure. My point is then that intracellular arcs, local multicellular arcs, and what we may call general axial arcs may all exist in the same individual, and so far as nervous development and differentiation is concerned, all be equally primitive. It is not necessary to assume that nervous structure was at first completely diffuse and equally distributed in all parts and that centralization occurred only in the course of evolution. If the physiological gradients in the body constitute the basis of the localization of nervous structure, we must expect to find more or less nervous centralization in any axiate animal in which nervous structure differentiates. A completely uncentralized system is possible only when axiate pattern as regards the body as a whole is absent, but, as a matter of fact, all animals in which a nervous system differentiates are more or less axiate. Whether the unicellular arcs, the local, or the general axial arcs are the more important in a particular case must depend upon the degree of development of physiological axiation and so of physiological integration in the body as a whole. In the medusa, a motile form, the development of axiation is higher and the general arcs as represented by the marginal sense organs, the ganglionic aggregation, and the nerve net are evidently more highly developed 
than in the sessile coelenterates. It may be pointed out, however, that in the sessile coelenterates there is normally a reversal of polarity during development. The planula possesses a well-marked apico-basal gradient, but as the period of attachment approaches this gradually disappears, and finally it is the original apical end which becomes attached, and the sessile individual arises as a new axial gradient, essentially a bud, at the original basal end. Moreover, in these sessile forms axiation is never very highly developed. Again in the sponge, which Parker regards as representing the primitive condition, the early developmental motile stage possesses a definite physiological axis, i.e., a primitive physiological gradient exists, but this disappears more or less completely during the later development, and the sessile adult exhibits an extremely low degree of physiological axiation and integration, so that practically nothing but local or unicellular arcs exist. But the sessile sponge and the sessile coelenterate develop from motile forms and the almost anaxiate condition of the adult is not primitive but secondary. Looking at the matter from this point of view, it is scarcely possible to agree with Parker, either that the contractile cells of the sponge are pure effectors or that the conditions in the sponges and sessile coelenterates represent the first step in the evolution of the receptor-conductor-effector system in free-living forms. They appear rather as special cases in which a form originally free-swimming and axiate has become sessile and almost anaxiate. Corresponding to the very low degree of integration in the adult sponge no distinguishable nervous structure is present, and in the unicellular or local arcs only the 
effector portion is visibly differentiated. In the actinian some degree of axial integration is undoubtedly present though it may be slight, as Parker's experiments indicate.

If the free-swimming sponge larva developed into a free-swimming adult instead of becoming sessile, there is no doubt that the axiation and physiological integration would progress instead of disappearing to a large extent and that one factor of this progressive development would be some sort of central nervous system. In such a case the local excitation arcs consisting of one or few cells would be more or less completely subordinated to the great general arcs of the chief axis. In short, the course of development of the excitation arc and the structures which represent it must be different in a form which remains motile throughout life from that in a form which becomes sessile after an early motile stage. In a free-living form nervous differentiation may occur as early as, or even earlier than, the differentiation of effectors, and this condition may be just as primitive or even more so than in the sponge, for since the nervous system is so intimately associated with motility we should scarcely look for the most primitive conditions in forms which are secondarily sessile.

Histologically, the condition in the sponges certainly supports the views of Claus and Chun that muscles and nerves originate independently and of course the embryonic differentiation of muscles in complete independence of nervous connections in the higher animals may be regarded as pointing to the same conclusion, but the origin and differentiation of muscles as of other organs is unquestionably associated with physiological 
gradients or excitation arcs, either local or general or both, and excitation and its transmission occur in protoplasm where no visible nerve structure exists.

Finally, the difficulty of conceiving a nervous system without effectors which was pointed out by Samassa and Schaeppi is a real difficulty, but when we remember that primitive protoplasm is effector as well as receptor and conductor and that passage of excitation over any protoplasmic region involves reception, conduction, and effect, this difficulty remains only for the teleological point of view. When we consider the nervous system as an adaptation for biological purposes, its existence without adapted effectors is difficult or impossible to conceive, but when we consider it as the visible expression and record in protoplasm of an excitation gradient or gradients resulting primarily from a differential relation to the external world, the presence or absence of a specialized effector becomes a matter of minor importance. In the first case we are considering the nervous system as a means, and without relation to the end-the effector or the effect produced by it-it becomes inexplicable, while in the second case we are considering it as an effect or result, and as such it follows necessarily from the irritability of protoplasm, the gradients, the capacity for morphological differentiation, etc.

\section{THE FUNCTIONAL DIRECTION IN THE NERVE NET}

The question of the localization of receptor, conductor, and effector and of what may be termed the functional direction of the reflex arc with respect to the axial gradients remains to be considered. As 
regards the coelenterates, the work of Parker on actinians and Corymorpha and the work of others on medusae ${ }^{\mathrm{r}}$ indicate that in these forms nervous transmission is highly diffuse and may occur in all directions from most or all regions of the body in the actinians and at least of the subumbrellar surface in the medusae. The experimental data do not indicate any differences in conductivity or range in any direction with respect to the body axis. I believe, however, that crucial experiments bearing upon this point have not yet been performed. Parker's experiments on the actinians, for example, are not adapted to show whether axial differences in irritability, conductivity, and range are present or not. For this purpose stimuli graded in intensity would be necessary, and aquatic forms are not favorable material for work of that kind. Mayer and Harvey have shown that impulses may apparently continue to travel indefinitely without any indication of decrement in circular strips of the medusa, Cassiopea, from which the sense organs have been removed, but they have not determined whether there is any difference in conductivity in the direction away from and toward the sense organs. There can be no doubt that in the medusae the sense organs are the most irritable regions and therefore in nature usually the initiators of impulses.

In the free-swimming medusae the specialization of both receptors and effectors is far more advanced than in the sessile coelenterates, and the whole receptorconductor-effector mechanism is much more highly

${ }^{x}$ Eimer (I878), Harvey (1912), Mayer (1906, I908, I916), Parker

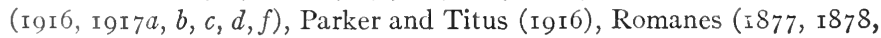
I893). 
developed on the apical body surface than elsewhere. Other polar specialization is not very great, consequently we need not expect to find any very marked difference between general axial arcs and others, except perhaps in the elongated manubria of certain species and in the tentacles, and in these organs we do find evidence of more or less definite axial arcs (see p. I I2).

It has been generally maintained that some degree of structural axiation exists in the nervous system of the sea anemones and corals and probably in that of the hydroids. According to this view, there is a more or less distinct aggregation or a greater development of nervous tissue in the apical region in relation to the mouth and tentacles. Wolff's (1903) figures of the general plan of the nervous system of the hydroid and the coral polyp indicate a considerable degree of axiation and centralization, but these figures, while based on the data of observation, are admittedly diagrammatic. If any degree of axial localization of nervous tissue and of receptors does exist in these sessile coelenterates, and the facts permit little doubt on that point, some degree of functional axiation undoubtedly exists in the excitation arcs, even though experimental stimulation not quantitatively controlled may show conduction occurring in all directions.

Many facts indicate that in these forms the receptor function is most highly developed in the apical regions; probably in the tentacles and about the mouth, and these regions are normally most exposed to excitation. In the tentacles the excitation arcs are as already noted, predominantly basipetal in both structure and function (pp. II 2, 203), and there can be no doubt that 
in the body as a whole there is some degree of apicobasal integration, with the apical region acting as receptor and dominant. In certain elongated actinians such as Cerianthus and Harenactis, which live in tubes in the sand with the apical end exposed, this is very clearly the case.

\section{FUNCTIONAL DIRECTION IN CERTAIN BILATERAL}

\section{INVERTEBRATES}

The functional direction of the arcs in forms with a synaptic nervous system is generally believed to be determinate and irreversible as regards the arc as a whole, although conduction supposedly occurs in both directions in the axon. The synapse, the rcgion of contact between two neurons, where passage of a cell membrane is involved in conduction of the impulse, is usually regarded as responsible in some way for this functional irreversibility of the arc (see pp. 228-32). The evidence for irreversibility, however, has been obtained chiefly from the higher vertebrates in which it appears to be a characteristic feature of the arc, but it is possible that even in these forms some paths in the central nervous system may be reversible as regards functional direction. If it is true that in the synaptic nervous system the reflex arc is functionally irreversible, then different or opposed functional directions must mean different paths. Certain conditions in some of the lower bilateral invertebrates are of interest as indicating the relation between functional direction and the chief axial gradient, though regarding the actual paths concerned in anterior and posterior directions we know practically nothing. 
It is in general true that in bilateral animals the chief receptors and the chief adjustor or central organ are situated in the head region, so that the functional direction of the reflex arcs is predominantly posterior, at least primarily. Attention has already been called to the fact that in various flatworms and annelids excitation is effective chiefly in the posterior direction. Stimulation of, or injury to, the head may produce effects throughout the whole length of the body, but stimulation of levels posterior to the head has relatively little effect anterior to the level of stimulation and much greater effect at more posterior levels. These facts indicate that the main conduction paths, or at least those which have to do with the body wall, function chiefly in the posterior direction, and that the head, so far as its relations to the rest of the body are concerned, is to a considerable extent autocratic. Unquestionably numerous local arcs exist in these forms, and it is by no means necessary to assume that the axial relations are the same for all organs of the body. As regards the alimentary tract of Planaria, for example, there is some evidence that the pharyngeal region, not the anterior end, is the dominant region, though whether nervous differentiation exists in the entoderm is not known. In most annelids the localization in early stages of a secondary growing region at the posterior end of the body determines a secondary gradient opposite in direction to the primary gradient and involving more or less of the posterior body region (Hyman, I916; Child I9I $7 d$ ). In many of the aquatic oligochaetes the posterior end is more or less dominant over the levels of this secondary gradient. The dominance of this 
posterior end over more anterior levels of the secondary gradient appears very clearly in the respiratory movement of the posterior body region when the animals are undisturbed. This movement of the body to and fro in the water is initiated at the posterior end, and that it is independent of the head is evident from the fact that it may occur in headless animals. But although this posterior region is ordinarily largely independent of the head, and the impulses in it apparently run predominantly in the anterior direction, a sufficient stimulation of the head, or in fact of any region of the body, brings this region more or less under the control of the head, the respiratory movement is inhibited, and the animals may move in a more or less co-ordinate manner. In these forms, then, the functional directions of the chief arcs are apparently opposite in anterior and posterior regions under conditions of slight stimulation but with suffciently intense excitation impulses may pass in either direction in all regions. In other words, the functional direction of the arcs which predominate under conditions of slight stimulation is down the gradients in both directions, but with strong stimulation impulses are conducted up as well as down. Paths upward are present, but a high degree of excitation is apparently necessary to make them available. To what extent or whether at all the upward and downward paths are identical is not known, though it appears not improbable that they may be identical over some portions of their length.

FUNCTIONAL DIRECTION IN THE HIGHER ANIMALS

While any extended survey of the conditions in the various groups is impossible at this time, it may be noted that with the progress of evolution the relation 
to the cephalic region of reflex arcs with receptors in postcephalic regions undergoes change. Apparently it becomes increasingly possible for excitation in such arcs to affect more anterior levels of the body and finally the cephalic region. In other words, the upward paths along the longitudinal axis apparently become more numerous and more readily traversed by impulses. It has been pointed out, for example (p. 99), that in the wormlike invertebrates even the excitation resulting from section of the body is much less effective at levels anterior than at levels posterior to the section, and less extreme excitations often produce no effect anterior but a marked effect posterior to the level of stimulation. The head region in such forms is to a considerable degree autocratic with respect to posterior levels. In the higher vertebrates, however, excitations from any level of the body may reach even the cerebral cortex, not directly, it is true, but only through the intermediation of various correlation centers. In the higher vertebrates and man, as compared with other forms, the upward paths in the longitudinal axis have apparently become more numerous and more readily traversed by impulses. Even in these forms, however, the upward paths in their definitive condition differ in general from the downward paths in that they usually consist of several neurons, while the downward paths consist of a smaller number or of only one. Functionally the definitive upward paths appear to present obstacles to the passage of impulses, while the downward paths are more simple and more open.

Special attention must be called in this connection to two facts: first, while this difference between upward 
and downward is characteristic of the definitive paths in the higher vertebrates, it is much less distinct in the lower vertebrates. The condition in mammals and man evidently represents the most advanced stage of an evolutionary process as regards these paths. Second, although our knowledge of the developmental changes in the nervous system, which lead up to this definitive condition, is fragmentary, certain facts at hand show that, in the Amphibia at least, the condition in early developmental stages is not only very different from the definitive condition in that group but more or less the opposite of the definitive condition in the higher vertebrates. Since the facts as they stand appear highly significant in relation to the physiological gradients and to the changes which they undergo in the course of development, a brief survey of certain of them is essential.

In the course of anatomical and physiological studies of the development of the nervous system of salamanders, Coghill (I909, I9I3, I9I4) has found that in the earliest stages at which movement of the body in response to tactile stimulation is possible, a definite reflex mechanism has already been developed, which differs in certain ways, highly significant in the present connection, from the definitive mechanisms. This mechanism is discussed in a later paper by Herrick and Coghill (I9I5) and is indicated in Figure 70, taken from that paper. Certain large sensory cells in the cord (giant cells of Rohon-Beard) have given rise to outgrowths regarded by the authors as dendrites (see pp. 196-200), which reach both the skin and the muscles, and to other outgrowths (axons), which ascend the cord 
all the way to the posterior end of the medulla oblongata. In this region they connect with the dendrites of neurons, whose axons form a ventral commissure across the cord

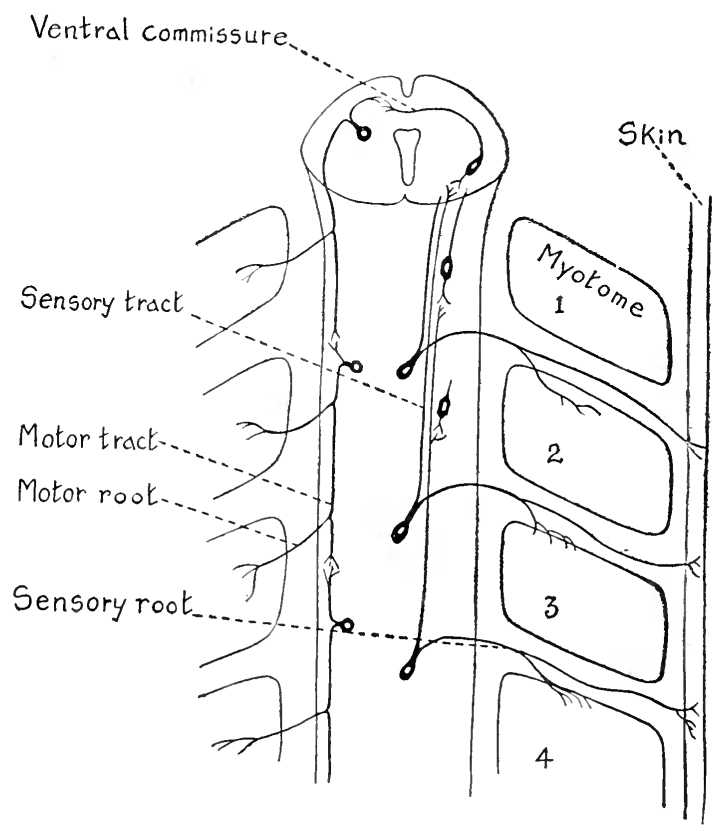

FIg. 70.-Diagrammatic dorsal view of the upper end of the spinal cord of larval Amblystoma, illustrating the relations of the giant cells, commissural cells, and motor cells and the mechanism of the swimming reflex (based on the researches of Coghill, I9I3, I9I4; from Herrick and Coghill, I915).

and connect with the motor neurons of the opposite side. These motor neurons send axons down the cord and collaterals to the muscles. At first the commissures occur only at the posterior end of the medulla, but in later stages the region of commissural connection. 
extends both anteriorly and posteriorly, until commissures are present at all levels posterior to the optic region.

The first motor response to tactile stimulation of regions posterior to the head is the contraction of muscles of the side opposite to that stimulated, first in the more anterior region just behind the head and later at more posterior levels, and the reflex mechanism indicated in Figure 70 constitutes the anatomical basis of this type of response. At this stage and in this the earliest reflex mechanism of the cord it is the upward path which is uninterrupted by synapses, while the downward path shows frequent interruptions of this kind and is apparently somewhat later in its development. In later stages the giant cells disappear, and other reflex mechanisms develop, until the definitive condition is attained.

These different characteristics of the upward and downward paths in the different vertebrate groups and in different stages of development of the individual must of course be dependent on differences in physiological conditions of some sort. The experimental data at hand concerning the physiological gradients in the vertebrates serve, I believe, to throw some light on these changes. It has already been pointed out (chap. viii) that in all segmented animals investigated, including annelids as well as vertebrates, a second region of high susceptibility and rapid growth arises early in development and gives rise to most or all of the postcephalic region. The presence of this second region of high metabolic rate has been demonstrated in fishes and in the chick by Hyman, whose work along this line is now 
in press, and in the frog by Bellamy (19rg). As the body develops, this region moves posteriorly and represents a posterior growing region of the embryo. Hyman has found that in the fish its susceptibility is equal to, or higher than, that of the head and much higher than that of more anterior postcephalic levels. Moreover, it is electronegative externally to all levels of the body behind the head. Bellamy has found that the susceptibility relations of this region in the frog are essentially similar to those in the fish, and the newly hatched tadpole shows a double gradient in susceptibility and electric potential, both susceptibility and external electronegativity decreasing from the posterior tail region to the body and from the head to the posterior body region, the posterior tail region showing at this time the highest electronegativity of any part of the body (unpublished). The reaction of the tadpole in a constant current, which consists in orientation with head toward the anode, instead of toward the cathode, as in most of the lower animals, is unquestionably dependent upon these physiological conditions. In still later stages of vertebrate development the posterior growing region disappears and with it usually the secondary gradient, though in fishes and in the tailed Amphibia it may persist to some extent in the tail region.

These facts show that there is a period of development in the vertebrates during which the longitudinal physiological gradient in the postcephalic region has its high end posteriorly and its low end anteriorly, i.e., just behind the head. Moreover, the presence of electrical differences associated with this gradient has been demonstrated in fishes and Amphibia. As regards the 
Amphibia, this is the period during which the reflex mechanism above described develops. In these experimentally demonstrated physiological axial relations we find, I believe, a simple basis for interpretation, not only of the remarkable reflex mechanism of early stages which Coghill has described, but of the progressive changes and the definitive condition. If the suggestions advanced in chapter xi concerning the significance of electrical polarization in determining neuron patterns are correct, there is no difficulty in understanding why axons growing down a general axial gradient are long and those growing in the opposite direction short. As I have suggested (p. I90), the growth direction of those growing up the general gradient is determined by local gradients arising from other neurons in the vicinity, or from other local conditions, and opposite in direction to the general gradient. The axon tip will remain positively polarized and will therefore grow only as far as the electrical factors of this gradient over-compensate those of the general gradient.

In the early stages of salamander development the upward sensory paths in the cord are growing down the general axial gradient as it exists at that time in that region, and they grow approximately to the level at which this gradient is compensated by the cephalic gradient. The downward motor path, on the other hand, is growing against the general gradient, and the length of each axon probably represents approximately the length at that time of the local gradient which determines it. In later stages, as this secondary developmental gradient becomes less marked, the sensory paths developing from the more posterior levels of 
the cord do not extend so far anteriorly but end at lower levels. Still later, as the growth activity and the internal electropositivity decrease at one level after another in the posterior direction and the gradient of the head region in the primary direction extends farther and farther posteriorly, this reflex mechanism of the earlier stages disappears and others take its place, following the gradient changes, until the definitive condition is attained. This case has been considered at some length because it seems highly significant. The appearance in the salamander of the early reflex mechanism so different in character from the definitive mechanisms and its complete disappearance later remain completely inexplicable facts, except in terms of the gradients as experimentally demonstrated. Viewed in relation to the gradients, however, these facts receive a simple physiological interpretation and fall into line with many others. Various other features of nervous development will probably be found to be related to the general or to local gradients or both, as they exist at the various stages.

In general a certain similarity exists between the structural and the functional characteristics of a path in relation to the gradients. This similarity appears in the differences between definitive upward and downward paths in man and other mammals. We may expect that a path which develops and functions up a general gradient will be made up of relatively short neurons and will show a high "resistance," while a path in the opposite direction will be relatively simple and open. Even the more primitive transmission paths exhibit a more or less definite functional direction with respect to physiological gradients present, excitation 
being effectively transmitted to greater distances down than up a gradient. If the electric current produced at a region of excitation is the transmitting factor this difference is readily understood (p. 97). As structural differentiation of transmission paths progresses, the morphological features of this relation to the gradients become more and more evident, until the conditions found in the higher animals and man are attained. Moreover, as regards the functional relations of neurons, even if we admit that conduction may occur without decrement in the single axon, the probability exists of quantitative differences in excitation in different neurons and of the relation of these differences to levels of the gradients and to the changes in the gradients which occur during development and evolution.

These suggestions do not involve the assumption that the growth of the axons is determined by the passage of nervous impulses, but if the suggestions advanced in chapters iv and xi have any basis in fact, the growth of the axon and the transmission of excitation are both determined by the electrical factor. The growth of the axon is the physiological effect of increase in internal positivity produced by the electric currents in a physiological gradient, and transmission is the physiological effect of the electrical currents associated with a local excitation, this effect being primarily, according to Lillie, an increase in internal positivity. From this viewpoint the outgrowth of the axon and the transmission of excitation are simply reactions of protoplasm to essentially the same electrical conditions, and it is not strange that they should exhibit certain similarities. 
Whether the paths from postcephalic regions upward are, in the higher vertebrates, parts of the main paths of the reflex arcs, as Pike (I909, I9I2) maintains, or are lateral or accessory paths not essential to the functioning of the arcs, as has been generally believed, is of secondary importance in this connection. The upward paths exist, and their existence suggests that the general anteroposterior physiological gradient in the central nervous system has become in some way less effective in determining functional direction in the higher than in the lower animals, and that the primitive relation of dominance and subordination with the more or less complete autocracy of the apical region or head in flatworms or annelids is undergoing alteration toward a relation of true co-ordination in which many different arcs with receptors in various regions of the body are concerned in determining the final effect or action. The physiological dominance of the nervous system and of more anterior over more posterior levels is in fact departing from the primitive, autocratic relation and becoming more and more like that of a representative government. Such change does not mean a decrease in the degree of physiological integration but rather an increase in the complexity, flexibility, and potentialities of integration. It does not represent a functional degradation of the higher levels but a functional elevation of the lower levels of the gradients, and this constitutes a basis for relations and combinations of arcs and paths which are impossible in the lower forms.

Such a decrease in functional or apparent steepness of the physiological gradient must be associated with the development and evolution of the mechanism of 
excitation in protoplasm. Certainly the intensity of the excitation process increases in general in development and evolution, and such change undoubtedly determines more effective transmission or conduction, just as a rapid change in electric potential is more effective than a slow change in producing excitation. It is not improbable also that in the more highly specialized excitatory processes the electrical changes are greater than in the primitive processes, and this again must contribute to more effective transmission. Other changes may also be concerned, but the point which I wish to make is that the changes are such as to make it possible for conduction of a local excitation to occur to a greater distance up a physiological gradient in the higher animals than in the lower. If the conception of excitation advanced in earlier chapters is correct, the change is probably primarily electrical.

It may be also that the gradients themselves are less steep in the higher than in the lower forms, but as yet no adequate basis exists for comparison of steepness in different forms, though the electrical data will probably give some evidence along this line. Finally, the fact that the higher animals consist of segments and in connection with segmentation develop a secondary posterior growing region and a secondary longitudinal gradient in the opposite direction to the primary gradient is unquestionably a factor of great importance in determining that the more posterior levels of the body are able to send impulses all the way to the head. This gradient determines the primary reflex mechanism in Amphibia, as already pointed out, and the definitive relations arise later after the gradients have undergone 
changes. The segmented animal does not represent the simple axial gradient of the lower forms, but is the result of a repetitive process of reproduction (p. I3 I). In early development a partial breaking up of the primary individual occurs and reintegration takes place only gradually as the segments successively become less active and the head region regains some degree of dominance. Under such conditions the relations of the different axial regions and of the different segments become much more complex than in the simple individual, and the possibility of impulses from posterior regions reaching the head is much greater. Segmentation of the body is then also a factor in the development of the more complex and more flexible type of physiological integration characteristic of the vertebrates.

THE PROBLEM OF THE ORIGIN AND DEVELOPMENT OF THE CEREBRAL CORTEX

It appears probable that the conditions determining the increase in number and functional significance of the upward paths in the central nervous system in the higher animals are in some way and to some degree concerned in the origin and development of the cerebral cortex. The cortex is not simply a central or adjustor organ for the receptors of the head region, as the cephalic ganglia of the invertebrates appear in large measure to be. It does not properly belong to the original reflex arcs at all, but represents functionally an additional arc superimposed upon the adjustor portion of the original arc. It may be termed a super-adjustor, but it is not simply that, for super-adjustors exist in the subcortical correlation centers. The cortex is in fact the supreme 
adjustor. This, however, does not mean that it is physiologically dominant in the more or less autocratic manner in which the head or the apical end of the physiological gradient is dominant. The cortex resembles rather a deliberative assembly to which reports of matters requiring consideration come in from the various constituent groups or bureaus and. in which they are considered and action finally taken through the proper channels (cf. Herrick, 1913, p. 230). Not every afferent impulse reaches the cortex. In many, and particularly postcephalic arcs, as long as the reflex mechanism of lower centers gives satisfactory results the cortex does not become involved; only when the stimulus continues and summation, or some other effect, makes it possible is the impulse able to reach the cortex.

As Herrick has so clearly pointed out:

There are no afferent tracts leading to the cerebral cortex directly from any peripheral sense organ or from any center within the brain which is "pure," i.e., devoted to a single sensory function. In other words, no simple sensory impulses ordinarily reach the cortex, but only nervous impulses arising from lower correlation centers, where complex reflex combinations of various sensory systems are possible. The optic impulses reach the cortex most nearly pure, i.e., with less subcortical associational relation with other sensory systems (it is no accident that the visual sense plays a dominant rôle in human cortical function); but even here the optic centers in the thalamus from which the optic projection fibres arise are intimately related with acoustic, tactile and other important sensory centers. And in the case of all the other sensory systems, the projection fibers which enter the cortex come from centers which are separated from their respective sense organs by two or more association centers of a high order of complexity. Each of these subcortical associational centers may be dominated physiologically by a single sensory system, but it is structurally adapted for bringing that system into relation with 
several others, so that the nervous discharge which emanates from it may be the efferent link in a very complex reflex arc. This efferent discharge may descend to the appropriate motor center, or it may ascend to enter a still higher association center, all of whose afferent tracts come from similar lower centers and therefore carry nervous impulses which represent a sort of physiological resultant of the functional factors there interacting [Herrick, I9I3, pp. 223-24].

The cortex represents in fact a region into which impulses originating in any receptor may under proper conditions enter, but only after passing through one or more correlation centers. Physiologically speaking, it is the "central" nervous system par excellence in that it is in a sense the meeting place of impulses from the most various sources.

The physiological conditions concerned in the origin and development of the cortex must be conditions which make possible or determine the localization of such a truly central nervous region. Among these conditions, those which have led to the development of the upward paths discussed in the preceding section must have been important factors. It has been suggested in chapter xi that the primary directive factors in development of the nervous system are electrical, arising in connection with the physiological gradients and their modifications and producing physiological effects through polarization, and if that is true it is allowable to suggest that similar electrical factors acting in the same way may be the chief or the primary physiological factors concerned in the ontogenetic localization and development of the cortex.

Gradients in electric potential are characteristic features of the primary physiological gradients and 
undergo modification with the other features of the gradients. The cerebral cortex apparently arises in organisms in which the primary gradients become so modified in the course of development that a truly central region is localized, which may be affected to some extent by changes of potential in any part of the organism. This being the case, the electrical situation in the region from which the cortex develops must be characteristically different in some way from that in other regions. More specifically stated, this region may be conceived as a region which represents not merely a certain level in the general axial gradients but which is affected, for example, by the electrical changes connected with localized developmental activity in various organ primordia, first of all the receptors of the head. Later, as the changes which have been briefly discussed in the preceding section occur, as various parts of the nervous system successively become functional in a specifically nervous way and as the upward paths in the cord develop, progressive modification and complication must occur.

The cortex represents a region which differs from others, not in kind, but in the variety and complexity of the structural and functional relations between its parts, and these relations must be determined by physiological factors of the same sort as those concerned in other parts of the nervous system, but acting under less simple conditions. If the conclusions and suggestions of earlier chapters concerning the part played in the development of the nervous system by electrical factors through their effects on physiological condition are correct, the physiological factors concerned in the 
development of the cortex must be in large part electrical in origin. The anatomical and functional relations of the cortex paths indicate clearly enough that conditions there are unique as regards complexity, though not different in kind from those in other parts, and it is difficult to conceive any other than electrical factors which could determine these relations. If we could trace out the electrical gradients and their changes and the sequence in which different body regions and organ primordia affect the cortex region electrically, either through developmental activity or functional excitation and so bring about physiological changes in this region, we might attain some conception of the basis for its remarkable structure and function.

The evolutionary aspect of the origin and development of the upward paths and of the cortex is of course another matter, involving, as I have repeatedly pointed out, the hereditary mechanism of the protoplasm concerned. In other words, the gradient situation works out differently in different protoplasms, and in those in which it works out in certain ways a cerebral cortex and the other features associated with it appear. Whether the evolutionary process requires Lamarckian assumptions or not does not primarily concern us here, but the gradient conception does not make such assumptions necessary. However the necessary mechanism of protoplasm is established, the development of the individual is a physiological process, and real advance in our conceptions of heredity is possible only as our knowledge of the physiology of development increases. The conception of the physiological gradient and of its relation to development and integration places these problems 
of developmental physiology in a new light, and I have ventured to suggest that even the more complex aspects of nervous development and integration are related to the fundamental physiological features of organismic pattern, the gradients.

From this point the attempt might be made to push suggestion and speculation along these lines into the field of psychology, but we are concerned primarily with the origin of the nervous system rather than with its relations to psychological phenomena. It seems allowable, however, to point out the possibility that the field or content of consciousness at any given moment may be associated with the determination of a field or area of relatively intense physiological activity in a particular region of the cortex as the resultant of the incoming impulses at that moment. If this activity is sufficiently intense, this area becomes for the time being the dominant region and its connections with other parts, together with the physiological conditions in other parts, determine the course of action. Hesitation, deliberation, delays, so characteristic of cortical reaction and consciousness, represent the period before complete dominance is attained and can be interpreted in terms of physiological factors. I do not mean to imply that each particular content of consciousness is localized in a particular cortical area, but merely that increased activity in a particular cortical region or regions is itself a resultant of a particular functional complex in the nervous system and the body as a whole, and that if such activity becomes sufficiently intense it determines a certain reaction complex. The structure of the cortex indicates that a general physiological 
axiation of the cortex as a whole is absent or practically absent, although a very definite surface-interior pattern does exist. The dominant region of the cortex may therefore shift from moment to moment and is purely physiological and transitory rather than morphologically persistent. That there is anything essentially new in these suggestions I should be the last to claim. They are brought forward here merely as the final point in the discussion of functional direction in relation to the physiological gradients. 


\section{CHAPTER XIV}

\section{GENERAL CONCLUSIONS}

In the preceding chapters an attempt has been made to show the physiological relation and continuity between the structural and functional development of the nervous system and the primary factors in organismic pattern, the physiological gradients. From the viewpoint which underlies the book the organism is regarded as representing essentially a behavior pattern, arising in protoplasm in response to its relations with the external world, and particularly the relations concerned in excitation and its effects. When we take all the facts into consideration, it is impossible, I believe, to reach any other conclusion than that the organism is such a pattern. The organism is without significance or meaning of any kind, except in relation to an external world, and particularly to the factors in that world which bring about excitation. I have endeavored to show that organismic pattern is primarily excitatory and transmissive in character, and that the material exchange between protoplasm and environment is of secondary importance, so far as this pattern is concerned, though necessary of course for the continued existence of protoplasm.

From this point of view the nervous system represents physiologically the development and complication of these fundamental excitatory features of organismic pattern and their transmissive effects in those protoplasms so constituted that such development is possible. Anatomically, the nervous system is the record in those 
protoplasms capable of developing and maintaining such a record, of the effects of the excitatory and transmissive processes upon the protoplasm. No other viewpoint yet attained affords any basis for the interpretation in physiological terms of the origin and development of the nervous system. If we maintain that organismic pattern is not fundamentally a relation of excitation and transmission, the appearance of the nervous system in development as the first definitive organ and its function as the chief organ of integration and relation in the organism must remain scientifically completely insoluble problems. Such a conception of the organism, carried to its logical conclusion, practically forces us to regard the nervous system as functionally and structurally something new appearing in the organism at a certain stage and without any pre-existing physiological basis. In other words, from this viewpoint the nervous system appears essentially as a special creation superimposed upon the organism and falls into the same category with Driesch's entelechy and with certain theological conceptions of the soul.

Such metaphysical difficulties are, however, avoided when we regard the nervous system as representing, on the one hand, the result and product of the primary excitation-transmission relations of organismic pattern, and, on the other, the further development of these relations. This conception gives us physiological continuity from the beginning of the organism to the highest stages of nervous development attained in the individual, or, stated in still broader terms, from the simple physiological gradient to the ego. The dominance of the nervous system and its integrating function 
in its various stages of development, up to the cerebral cortex of man, is in these terms a physiological consequence of the establishment of the physiological gradient or gradients in the cell or cell mass from which the individual develops.

The distinction between pattern and material, between the physiological gradient and the hereditary constitution of the protoplasm in which it arises, has been pointed out repeatedly and may be emphasized once more. The course of events in relation to a gradient, for example, the particular kind of nervous system which develops in a particular organism is primarily a matter of hereditary constitution, but the gradients determine how the potentialities of the protoplasm as regards nervous development shall be realized.

This conception of organismic pattern and of the relation of nervous pattern to it is fundamentally a physiological conception and requires no assumptions nor special hypotheses concerning heredity and evolution, except in so far as it involves rejection of the more extreme preformistic types of theory. It does not, however, in any sense represent the extreme epigenetic viewpoint. Moreover, it does not, as some of its critics have asserted, involve acceptance of Lamarckian doctrine, though it is possible of course to develop a Lamarckian conception of inheritance of organismic pattern from the conception of the physiological gradient. The gradient is primarily nothing more than an ordering and integrating factor in developmental physiology.

Objection may be, in fact has been, made to the use of the conception of the physiological gradient as the foundation of a theory of organismic and nervous pattern 
and integration on the grounds that, while various lines of evidence indicate the existence of these gradients, we do not yet know what they really are. As a matter of fact, we do not know what anything in the organism really is; we merely know more or less about it. Up to the present we have learned many things about the physiological gradients and many more remain to be learned. We can state, however, on grounds much more adequate than those on which many other scientific conclusions rest, that these gradients are primarily quantitative in character, that while they may persist through reproduction, i.e., be inherited, they arise primarily in relation to a quantitative differential in the action of an external factor on protoplasm, that they show all the characteristics of excitation-transmission gradients, and, finally, that they are of fundamental significance in the development and physiological integration of the organism. The evidence for the existence of the physiological gradients and for their significance is based largely on experimental data and is therefore more definite and conclusive than much of the evidence on which various other current biological theories have been built up.

While experimental data concerning many features of nervous development are lacking, the main facts of its relation to the gradients are clear. As regards many points only suggestion, inference, or a weighing of probabilities is at present possible, but it has seemed desirable in the preceding chapters to follow through the line of thought developing from the experimental foundation and therefore to advance suggestions, to consider probabilities, and to draw tentative conclusions in many 
cases. Such procedure is of value in two ways: first, it indicates to what extent the theory or working hypothesis may serve; and, second, the consideration of the various questions from a particular viewpoint may provide a basis for further investigation, which will confirm the suggestions or show them to be incorrect.

Questions of detail aside, the general conception on which the book is based involves three main theses, which are, I believe, of fundamental importance for biological theory. These theses are: first, that development is a physiologically continuous process; second, that the problem of organismic pattern conceived in any other terms than those of relation to the external world becomes essentially a metaphysical rather than a scientific problem and it is necessary to postulate an entelechy, a soul, or some other metaphysical ordering and integrating factor; third, that in the excitatory relation between protoplasm and the external world and the effects of such excitation on protoplasm we have an adequate physiological basis for organismic pattern and for the physiological continuity of development. If the book contributes toward the establishment of these three theses as statements of fundamental biological fact, it will have attained its chief purpose. 
Allen, G. D.

\section{REFERENCES}

I9I9. "Quantitative Studies on the Rate of Respiratory Metabolism in Planaria, I, Amer. Jour. Physiol., XLVIII.

Alvarez, W. C.

I9I4. "Functional Variations in Contractions of Different Parts of the Small Intestine," Amer. Jour. Physiol., XXXV.

I9I 5a. "The Motor Functions of the Intestine from a New Point of View," Jour. Amer. Med. Assoc., LXV.

r915b. "Further Studies on Intestinal Rhythm, II," Amer. Jour. Physiol., XXXVII.

I9r6a. "IV. Differences in Rhythmicity and Tone in Different Parts of the Wall of the Stomach," Amer. Jour. Physiol., XL.

I9r6b. "V. Differences in Irritability and Latent Period in Different Parts of the Wall of the Stomach," Amer. Jour. Physiol., XLI.

r9I7a. "VI. Differences in Latent Period and Form of the Contraction Curve in Muscle Strips from Different Parts of the Frog's Stomach," Amer. Jour. Physiol., XLII.

I9I7b. "VII. Differences in Latent Period and Form of Contraction Curve in Muscle Strips from Different Parts of the Mammalian Stomach," Amer. Jour. Physiol., XLII.

I9I8a. "X. Differences in Behavior of Segments from Different Parts of the Intestine," Amer. Jour. Physiol., XLV.

i9I8b. "XII. The Influence of Drugs on Intestinal Rhythmicity," Amer. Jour. Physiol., XLVI.

I9I8c. "XVI. Differences in the Action of Drugs on Different Parts of the Bowel," Jour. Pharm. and Exp. Ther., XII. 
Alvarez, W. C., and Starkweather, E.

rgr8a. "XI. The Metabolic Gradient Underlying Intestinal Peristalsis," Amer. Jour. Physiol., XLVI.

I9I8b. "XIII. The Motor Functions of the Cecum," Amer. Jour. Physiol., XLVI.

I9I8c. "XVII. The Metabolic Gradient Underlying Colonic Peristalsis," Amer. Jour. Physiol., XLVII.

rgrg. "XVIII. Conduction in the Small Intestine," Amer. Jour. Physiol., L.

Alvarez, W. C., and Taylor, F. B.

rgr7a. "The Effect of Temperature on the Rhythm of Excised Segments from Different Parts of the Intestine," Amer. Jour. Physiol., XLIV.

I9I $7 b$. "Changes in Rhythmicity, Irritability and Tone in the Purged Intestine," Jour. Pharm. and Exp. Ther., X.

BARKER, L. F.

I9oI. The Nervous System and Its Constituent Neurons. New York.

BAyliss, W. M.

1918. Principles of General Physiology. Second edition. London.

Bellamy, A. W.

I9I9. "Differential Susceptibility as a Basis for Modification and Control of Early Development in the Frog," Biol. Bull., XXXVII.

Bethe, A.

1897. "Das Nervensystem von Carcinus maenas, I," Arch. f. mikr. Anat., L.

BiedermanN, W.

1896. Electro-Physiology. English translation. London.

r903. "Elektrophysiologie," Ergebn. d. Physiol., Jahrgang II, Abt. II.

BoK, S. T.

I9I 5. "Die Entwicklung der Hirnnerven und ihrer Zentralen Bahnen. Die stimulogene Fibrillation," Folia NeuroBiol., IX. 
Boruttau, H.

Igor. "Die Aktionsströme und die Theorie der NervenBose, J. C. leitung," Arch.f.d.ges. Physiol., LXXXIV.

1902. Response in the Living and Non-Living. London.

I906. Plant Response. London.

I907. Comparative Electro-Physiology. London.

I9I3. Researches on Irritability. London.

BOVERI, T.

I9ro. "Die Potenzen der Ascaris-Blastomeren bei abgeänderter Furchung," Festschr. zum sechzigsten Geburtstage R. Hertwigs, III.

Bresslau, E.

I904. "Beiträge zur Entwicklungsgeschichte der Turbellarien, I," Zeitschr. f. wiss. Zoöl., LXXVI.

BrüCKe, E. T. VON.

I9I6. "Versuche an ausgeschnittenen und reimplantierten Flimmerschleimhautstücken," Arch. f. d. ges.

CAJAL, S. R. Physiol., CLXVI.

I89o. "A quelle époque apparaissant les expansions des cellules nerveuses de la moelle épinière du poulet," Anat. Anz., V.

1909. Histologie du système nerveux de l'homme et des vertèbres. Vol. I. Paris.

I9I I. Histologie, etc. Vol. II. Paris.

Child, C. M.

I904. "Studies on Regulation, V, VI," Jour. Exp. Zoöl., I. I907a. "An Analysis of Form Regulation in Tubularia, I," Arch.f. Entwickelungsmech., XXIII.

I907b. "An Analysis, etc., II," Arch. f. Entwickelungsmech., XXIII.

1907c. "An Analysis, etc., IV," Arch.f. Entwickelungsmech., XXIV.

1907d. "An Analysis, etc., V," Arch. f. Entwickelungsmech., XXIV.

I909. "Factors of Form Regulation in Harenactis attenuata, III." Jour. Exp. Zoül., VII. 


\section{Child, C. M.}

rgroa. "Physiological Isolation of Parts and Fission in Planaria," Arch.f. Entwickelungsmech., XXX, II Teil. rgrob. "Further Experiments on Adventitious Reproduction and Polarity in Harenactis," Biol. Bull., XX.

rgria. "Experimental Control of Morphogenesis in the Regulation of Planaria," Biol. Bull., XX.

rgr $b$. "Studies on the Dynamics of Morphogenesis and Inheritance in Experimental Reproduction, I," Jour. Exp. Zoöl., X.

I9 I c. "Studies, etc., II," Jour. Exp. Zoöl., XI.

I9I Id. "Studies, etc., III," Jour. Exp. Zoöl.. XI.

igr ie. "Die physiologische Isolation von Teilen des Organismus," Vortr. u. Aufs. ü. Entwickelungsmech., Heft XI.

I9r2a. "Studies, etc., IV," Jour. Exp. Zoöl., XIII.

I9г2b. "Correlation in Regulation," Proc. 7th Intcrnat. Zoöl. Congress, Boston, r907. Published rgr2.

r9гза. "Studies, etc., V," Jour. Exp. Zoöl., XIV.

I9I3b. "Studies, etc., VI," Arch. f. Entwickelungsmech., XXXVII.

r9r4a. "Susceptibility Gradients in Animals," Science, XXXIX.

I9I4b. "The Axial Gradient in Ciliate Infusoria," Biol. Bull., XXVI.

I9I4c. "Starvation, Rejuvenescence and Acclimation in Planaria dcrotocephala," Arch. f. Entwickelungsmech., XXXVIII.

I9I4d. "Studies, etc., VII, Jour. Exp. Zoöl., XVI.

igr4e. "Studies, etc., VIII," Jour. Exp. Zoöl., XVII.

I915a. "Axial Gradients in the Early Development of the Starfish," Amer. Jour. Physiol., XXXVII.

r9i5b. Senesconce and Rejuvenescence. Chicago.

I9r 5c. Individuality in Organisms Chicago.

I9r6a. "Axial Susceptibility Gradients in the Early Development of the Sea Urchin," Biol. Bull., XXX.

rgr6b. "Studies, etc., IX," Jour. Exp. Zoöl., XXI.

rgr6c. "Axial Susceptibility Gradients in Algae," Bot. Gaz., LXII. 
ChIld, C. M.

IgI6d. "Experimental Control and Modification of Larval Development in the Sea Urchin in Relation to the Axial Gradients," Jour. Morphol., XXVIII.

I916e. "Further Observations on Susceptibility Gradients in Algae," Biol. Bull., XXXI.

I9I7a. "Susceptibility Gradients in the Hairs of Certain Marine Algae," Biol. Bull., XXXII.

I9I 76 . "Experimental Alteration of the Axial Gradient in the Alga, Griffithsia bornetiana," Biol. Bull., XXXII.

I9I7c. "The Gradient in Susceptibility to Cyanides in the Meridional Conducting Path of the Ctenophore, Mnemiopsis," Amer. Jour. Phsyiol., XLI.

I9I $7 d$. "Differential Susceptibility and Differential Inhibition in the Development of Polychaete Annelids," Jour. Morphol., XXX.

1918. "Physiological Senescence in Hydromedusae," Biol. Bull., XXXIV.

I9Iga. "Demonstration of the Axial Gradients by Means of Potassium Permanganate," Biol. Bull., XXXVI.

Igrgb. "The Axial Gradients in Hydrozoa, II," Biol. Bull., XXXVII.

I9I9c. "The Effect of Cyanides on Carbon Dioxide Production and on Susceptibility to Lack of Oxygen in Planaria dorotocephala," Amer. Jour. Physiol., XLVIII.

I9I9d. "Susceptibility to Lack of Oxygen During Starvation in Planaria," A mer. Jour. Physiol., XLIX.

19Ige. "A Study of Susceptibility in Some Puget Sound Algae," Pub. Puget Sound Biol. Sta., II.

1920. "Studies on the Dynamics of Morphogenesis and Inheritance in Experimental Reproduction," $\mathrm{X}$, Jour. Exp. Zoöl., XXX.

Child, C. M., and Bellamy, A. W.

1919. "Physiological Isolation by Low Temperature in Bryophyllum and Other Plants," Science, L.

ChILd, C. M., and Hyman, L. H.

rgrg. "The Axial Gradients in Hydrozoa, I," Biol. Bull., XXXVI. 
Chrld, C. M., and McKie, E. V. M.

rgr I. "The Central Nervous System in Teratophthalmic and Teratomorphic Forms of Planaria dorotocephala," Biol. Bull., XXII.

Chun, C.

r880. "Die Ctenophoren des Golfes von Neapel," Fauna

Ciaus, C. und Flora des Golfes von Neapel. Monogr. I.

I878. "Studien über Polypen und Quallen der Adria," Denkschr. d. Akad.d. Wiss. Wien., XXXVIII.

Coghill, G. E.

r909. "The Reaction to Tactile Stimuli and the Development of the Swimming Movement in Embryos of Diemyctylus torosus Eschscholts," Bull. of Denison Univ., XIV.

I913. "The Primary Ventral Roots and Somatic Motor Column of Amblystoma," Jour. Comp. Neurol., XXIII.

r9r4. "Correlated Anatomical and Physiological Studies of the Growth of the Nervous System of Amphibia, I," Jour. Comp. Neurol., XXIV.

DEWEy, J.

I893. "The Reflex Arc Concept in Psychology," Psychol. Rev., III.

DuCCESCHT, V.

I90I. "Über die Wirkung engbegrenzter Nervencompression," Arch.f.d.ges. Physiol., LXXXIII.

EIMER, T.

1878. Die Medusen, physiologisch und morphologisch auf ihr Nervensystem untersucht. Tübingen.

Fischel, A.

I9r4. "Über das Differenzierungsvermögen der Gehirnzellen," Arch.f. Entwickelungsmech., XL.

Fischer, A.

I9I1. "Ein Beitrag zur Kenntniss des Ablaufes des Erregungsvorgangs im marklosen Warmbluternerven," Zeitschr.f. Biol., LVI. 
Fitring, $\mathrm{H}$.

1907. "Die Reizleitungsvorgänge bei den Pflanzen," Sonderabdr. aus Ergebn.d.Physiol., IV und V. Wiesbaden.

FlexNer, $\mathrm{S}$.

I898. "The Regeneration of the Nervous System of Planaria torva and the Anatomy of Double-Headed Forms," Jour. Morphol., XIV.

GoldFARB, A. J.

1909. "The Influence of the Nervous System in Regeneration," Jour. Exp. Zoöl., VII.

GrošELJ, P.

I909. "Untersuchungen über das Nervensystem der Aktinien," Arb. Zoöl. Inst. Wien., XVII.

Grützner, P.

r882. "Zur Physiologie des Flimmerepithels," Physiol. Studien. Leipzig.

HARRISON, R. G.

1908. "Embryonic Transplantation and the Development of the Nervous System," A nat. Rec., II.

1910. "The Outgrowth of the Nerve Fiber as a Mode of Protoplasmic Movement," Jour. Exp. Zoöl., IX.

19I8. "Experiments on the Development of the Fore Limb of Amblystoma, a Self-differentiating Equipotential System," Jour. Exp. Zoöl., XXV.

Harvey, E. N.

I912. "The Question of Nerve Fatigue," Carnegie Inst. Yearbook, No. Io.

HERRICK, C. J.

1905. "The Central Gustatory Paths in the Brains of Bony Fishes," Jour. Comp. Neurol., XV.

1913. "Some Reflections on the Origin and Significance of the Cerebral Cortex," Jour. Animal Beh., May-June.

rgr8. An Introduction to Neurology. Second edition. Philadelphia.

Herrick, C. J., and Coghill, G. E.

I9I 5. "The Development of Reflex Mechanisms in Amblystoma," Jour. Comp. Neurol., XXV. 
Hertwig, O. and R.

I878. Das Nervensystem und die Sinnesorgane der Medusen. Leipzig.

Hertwig, R.

1905. A Manual of Zoölogy. English translation. New York.

Hils, A. V.

I9I2. "Absence of Temperature Changes During the Transmission of a Nervous Impulse," Jour. Physiol., XLIII.

HiLl, C.

I900. "Developmental History of the Primary Segments of the Vertebrate Head," Zoöl. Jahrbiicher, Abt. f. Anat. und Ont., XIV.

HIs, W.

I889. "Die Neuroblasten und deren Entstehung im embryonalen Mark," Arch. f. Anat. u. Entwicklungsges., Jahrgang I889.

Hyde, IDA $\mathrm{H}$.

I904. "Differences in Electrical Potential in Developing Eggs," Amer. Jour. Physiol., XII.

HyMan, L. H.

I916. "An Analysis of the Process of Regeneration in Certain Microdrilous Oligochaetes,"Jour.Exp.Zoöl., XX.

I9I 7. "Metabolic Gradients in Amoeba and Their Relation to the Mechanism of Amoeboid Movement," Jour. Exp. Zoöl., XXIV.

I9I8. "Suggestions Regarding the Causes of Bioelectric Phenomena," Science, XLVIII.

I9I9. "On the Action of Certain Substances on Oxygen Consumption. II. Action of Potassium Cyanide on Planaria," Amer. Jour. Physiol., XLVIII.

Johnson, Myrtle E. A.

r910. "Quantitative Study of the Development of the Salpa Chain in Salpa fusiformis-uncinata.," Univ. Cal. Pub., Zoöl., VI.

Johnston, J. B.

1906. The Nervous System of Vertebrates. Philadelphia. 
KAPpers, C. U. A.

I917. "Further Contributions on Neurobiotaxis. IX. An Attempt to Compare the Phenomena of Neurobiotaxis with Other Phenomena of Taxis and Tropism. The Dynamic Polarization of the Neuron," Jour. Comp. Neurol., XVII.

KEILLER, V. H.

I910. "A Histological Study of Regeneration in Short Head Pieces of Planaria simplicissima," Arch. f. Entwickelungsmech., XXXI.

KLeineneerg, N.

I872. "Hydra": eine anatomisch-entwicklungsgeschichtliche KNIEP, H. Untersuchung. Leipzig.

1907. "Beiträge zur Keimungsphysiologie und Biologie KRAFT, H. von Fucus," Jahrb.f. wiss. Bot., XLIV.

1890. "Zur Physiologie des Flimmerepithels bei den Wirbeltieren," Arch.f.d.ges. Physiol., XLVI.

KRETZSChMAR, P.

1904. "Über Entstehung und Ausbreitung der Plasmaströmung in Folge von Wundreiz," Jahrbiucher $f$. wiss. Bot., XXXIX.

LilLie, F. R.

IgOI. "Notes on Regeneration and Regulation in Planarians, II," Amer. Jour. Physiol., VI.

I9I9. The Development of the Chick. Second edition. New York.

LILLIE, R. S.

Igoga. "On the Connection between Stimulation and Changes in the Permeability of the Plasma Membrane of the Irritable Element," Science, XXX.

I $909 b$. "On the Connection between Changes of Permeability and Stimulation and on the Significance of Changes in Permeability to Carbon Dioxide," Amer. Jour. Physiol., XXIV.

Igogc. "The General Biological Significance of Changes in the Permeability of the Surface Layer of the Plasma Membrane of Living Cells," Biol. Bull., XVII. 
LILLIE, R. S.

I9II. "The Relation of Stimulation and Conduction in Irritable Tissues to Changes in the Permeability of the Limiting Membranes," Amer. Jour. Physiol., XXVIII.

I9I3. "The Rôle of Membranes in Cell Processes," Pop. Sci. Mo., February.

I9I4. "The Conditions Determining the Rate of Conduction in Irritable Tissues and Especially in Nerve," Amer. Jour. Physiol., XXXIV.

I91 5. "Conditions of Conduction of Excitation in Irritable Cells and Tissues and Especially in Nerve, II," Amer. Jour. Physiol., XXXVII.

I9I6a. "The Theory of Anesthesia," Biol. Bull., XXX.

I9I6b. "The Conditions of Physiological Conduction in Irritable Tissues, III," Amer. Jour. Physiol., XLI.

I917. "The Formation of Structures Resembling Organic Growths by Means of Electrolytic Local Action in Metals and the Physiological Significance and Control of that Type of Action," Biol. Bull., XXXIII.

I918. "Transmission of Activation in Passive Metals as a Model of the Protoplasmic or Nervous Type of Transmission," Science, XLVIII.

1919. "Nervous and Other Forms of Protoplasmic Transmission," Sci. Mo., May and June.

Lillie, R. S., and Johnston, E. N.

I9I9. "Precipitation Structures Simulating Organic Growths," Biol. Bull., XXXVI.

Locy, W. A.

I895. "A Contribution to the Structure and Development of the Vertebrate Head," Jour. Morphol., XI.

LoDHOLZ, E.

I913. "Das Dekrement der Erregungswelle im erstickenden Nerven," Zeitschr.f. allgem. Physiol., XV.

Loeb, J.

1892. Untersuchungen zur physiologischen Morphologie der Tiere. II. Würzburg.

I9r6. The Organism As a Whole. New York. 
LUCAS, K.

I9I7. The Conduction of the Nervous Impulse. London.

Lund, E. J.

I918. "Quantitative Studies on Intracellular Respiration, II," Amer. Jour. Physiol., XLV.

MacArthur, C. G., and Doisy, E. A.

I9I9. "Quantitative Chemical Changes in the Human Brain During Growth," Jour. Comp. Neurol., XXX.

MacArthur, C. G., and Jones, O. C.

I9I7. "Some Factors Influencing the Respiration of Ground Nervous Tissue," Jour. Biol. Chem., XXXII.

Mathews, A. P.

I903. "Electrical Polarity in Hydroids," Amer. Jour Physiol., VIII.

Mayer, A. G.

Igo6. "Rhythmical Pulsation in Scyphomedusae," Carnegie Inst. Publ. No. 47.

I908. "Rhythmical Pulsation in Scyphomedusae, II," Carnegie Inst. Publ. No. IOz.

1916. "Nerve Conduction and Other Reactions in Cassiopea," Amer. Jour. Physiol., XXXIX.

Morat, J. P.

I906. Physiology of the Nervous System. English translation. Chicago.

Morgan, T. H.

I901. "Factors That Determine Regeneration in Antenmularia," Biol. Bull., II.

1904. "An Attempt to Analyze the Phenomena of Polarity in Tubularia," Jour. Exp. Zoöl., I.

I906. "Hydranth-Formation and Polarity in Tubularia," Jour. Exp. Zoöl., III.

1907. Experimental Zoölogy. New York.

I9I9. The Physical Basis of Heredity. Philadelphia.

Morgan, T. H., and Dimon, A. C.

I904. "An Examination of the Problem of Physiological 'Polarity' and Electrical Polarity in the Earthworm," Jour. Exp. Zoöl., I. 
NEAL, H. V.

I898. "The Segmentation of the Nervous System in Squalus acanthias," Bull. Mus. Comp. Zoöl., XXXI.

I9I8. "Neuromeres and Metameres," Jour. Morphol., XXXI.

Norman, W. W.

1900. "Do the Reactions of the Lower Animals against Injury Indicate Pain Sensations?" Amer. Jour. Physiol., III.

PARker, G. H.

I905. "The Movements of the Swimming Plates in Ctenophores, with Reference to the Theories of Ciliary Metachronism," Jour. Exp. Zoöl., II.

I909. "The Origin of the Nervous System and Its Appropriation of Effectors," Pop. Sci. Mo., I909.

I9roa. "The Reactions of Sponges with a Consideration of the Origin of the Nervous System," Jour. Exp. Zoöl., VIII.

I9ıob. "The Phylogenetic Origin of the Nervous System," Anat. Rec., IV.

I9I I. "The Origin and Significance of the Primitive Nervous System," Proc. Amer. Phil. Soc., L.

I9I4. "The Origin and Evolution of the Nervous System," Pop. Sci. Mo., I9I4.

r9r6. "The Effector Systems of Actinians," Jour. Exp. Zoöl., XXI.

I9r 7a. "Nervous Transmission in the Actinians," Jour. Exp. Zoöl., XXII.

rgr7b. "The Movements of the Tentacles in Actinians," Jour. Exp. Zoöl., XXII.

I9I7c. "Pedal Locomotion in Actinians," Jour. Exp. Zoöl., XXII.

I9г7d. "Actinian Behavior," Jour. Exp. Zoöl., XXII.

I9I7e. "The Sources of Nervous Activity," Science, XLV.

I9I7f. "The Activities of Corymorpha," Jour. Exp. Zoöl., XXIV.

I9I8. "Some Underlying Principles of the Structure of the Nervous System," Science, XLVII.

I9I9. The Elementary Nervous System. Philadelphia. 
Parker, G. H., and Titus, E. G.

I9I6. "The Structure of Metridium (Actinoloba) marginatus Milne-Edwards with Special Reference to Its NeuroPike, F. H. muscular Mechanism," Jour. Exp. Zoöl., XXI.

I909. "Studies in the Physiology of the Central Nervous System, I," Amer. Jour. Physiol., XXIV.

I9I 2. "Studies, etc., II," Amer. Jour. Physiol., XXX.

Pringshein, E. G.

I91 2. Die Reizbewegungen der Pflanzen. Berlin. PütTer, A.

I903. "Die Flimmerbewegung," Ergebn. d. Physiol., JahrRAND, H. W. gang II, Abt. II.

I9I I. "The Problem of Form in Hydra," Science, XXXIII.

I9I 2. "The Problem of Organization," Science, XXXVI. REgel, F.

I876. "Die Vermehrung der Begoniaceen aus ihren Blättern," Jen. Zeitschr., X.

ReICHert, E. T., and Browns A. F.

I909. "The Differentiation and Specificity of Corresponding Proteins and Other Vital Substances in Relation to Biological Classification and Organic Evolution. The Crystallography of Hemoglobins," Carnegie

Retzius, G. Inst. Publ. No. II6.

I892. "Biologische Untersuchungen," Newe Folge, III.

RHuMBLER, L.

I905. "Aus dem Lückengebiet zwischen organismischer und anorganismischer Materie," Ergebn. d. Anat. u. Entwicklungsges., XV.

RIGGs, L. K.

I9I9. "Action of Salts upon the Metabolism of Nerve," Jour. Biol. Chem., XXXIX.

RitTER, W. E.

I909. "Halocynthia Johnsoni n. sp. A Comprehensive Inquiry As to the Extent of Law and Order That Prevails in a Single Animal Species," Univ. Cal. Publ., Zoöl., VI.

I9I9. The Unity of the Organism. Boston. 
Ritter, W. E., and Johnson, Myrtle

I9II. "The Growth and Differentiation of the Chain of Cyclosalpa affinis Chamisso," Jour. Morphol., XXII. Robbins, H. S., and Child, C. M.

1920. "Carbon Dioxide Production in Relation to Regeneration in Planaria dorotocephala," Biol. Bull., XXXVIII.

Rolianes, G. J.

I877. "Preliminary Observations on the Locomotor System of Medusae," Phil.Tr., CLXVI.

I878. "Further Observations on the Locomotor System of Medusae," Phil. Tr., CLXVII.

I893. Jellyfish, Starfish and Sea-Urchins. Internat. Sci. Ser., New York.

Samassa, $\mathrm{P}$.

I892. "Zur Histologie der Ctenophoren," Arch. mikr. Anat., $\mathrm{XL}$.

SCHAEPPI, $\mathrm{T}$.

I904. "Úber den Zusammenhang von Muskel und Nerv bei den Siphonophoren," Mitt. naturwiss. Ges. Winterthiir, Jahrgang I903-4.

SHERrington, C. S.

1906. The Integrative Action of the Nervous System. New York.

SHOREy, Marian L.

1909. "The Effect of the Destruction of Peripheral Areas on the Differentiation of the Neuroblasts," Jour. Exp. Zoöl., VII.

Stahl, E.

I885. "Über den Einfluss der Beleuchtungsrichtung auf die Teilung der Equisetumsporen," Berichte deutsch. bot. Ges., III.

Stevens, N. M.

I902. "Regeneration in Antenmularia ramosa," Arch. $f$. Entwickelungsmech., XV.

I9I0. "Regeneration in Antennularia," Arch. f. Entwickelungsmech., $\mathrm{XXX}$. 
STOCKARD, C. R.

I907. "The Artificial Production of a Single Median Cyclopian Eye in the Fish Embryo by Means of Sea Water Solutions of Magnesium Chloride," Arch.f. Entwickelungsmech., XXIII.

I909. "The Development of Artificially Produced Cyclopian Fish. The Magnesium Embryo," Jour. Exp. Zoöl., VI.

I910. "The Influence of Alcohol and Other Anesthetics on Embryonic Development," Amer. Jour. Anat., X.

I9I I. "The Experimental Production of Various Eye Abnormalities and An Analysis of the Development of the Primary Parts of the Eye," Arch. f. vergl. Ophthalmol., I.

TASHIRO, S.

I9I4. "The Metabolic Gradient in the Nerve Fiber," Proc. Amer. Physiol. Soc., Amer. Jour. Physiol., XXXIII.

I9I 5a. "The Metabolism of Resting Nerve and Its Correlation with the Direction and Rate of Nerve Impulse," Proc. Amer. Physiol. Soc., Amer. Jour. Physiol., XXXVI.

19I5b. "On the Nature of the Nerve Impulse," Proc. Nat. Aca. Sci., I.

I9I7. A Chemical Sign of Life. Chicago.

Taylor, F. B., and Alvarez, W. C.

I9I7. "The Effect of Temperature on the Excised Segments of Different Parts of the Intestine," Amer. Jour. Physiol., XLIV.

VERWORN, M.

I89o. "Studien zur Physiologie der Flimmerbewegung," Arch.f.d.ges. Physiol., XIVIII.

I9I3. Irritability. New Haven.

WAELSCH, L.

I914. "Über experimentelle Erzeugung von Epithelwuchserungen und Vervielfachungen des Medullarrohres (Polymyelie) bei Hühnerembryonen," Arch. $f$. Entwickelungsmech., XXXVIII. 
Waller, A. D.

1897. Lectures on Physiology. First Series. "On Animal Electricity." London.

1903. Signs of Life from Their Electrical Aspect. London. Wilson, E. B.

I895. "The Cell Lineage of Nereis," Jour. Morphol., VI. Wilson, H. V.

1907. "On Some Phenomena of Coalescence and Regeneration in Sponges," Jour. Exp. Zoöl., V.

I9I I. "Development of Sponges from Dissociated Tissue Cells," Bull. Bur. Fish., XXX.

WINKLER, $\mathrm{H}$.

I900a. "Úber Polarität, Regeneration und Heteromorphose bei Bryopsis," Jahrb.f. wiss. Bot., XXXV.

I900b. "Uber den Einfluss äusserer Faktoren auf die Teilung des Eies von Cytosira barbata," Berichte deutsch. bot. Ges., XVIII.

WolfF, M.

1903. "Das Nervensystem der polypoiden Hydrozoa und Scyphozoa," Zeitschr.f. allgem. Physiol., III. 
INDEX 


\section{INDEX}

Note-References give the number of the page on which the matter referred to begins.

Acceleration, differential, 37

Acclimation: in relation to physiological condition, $3 \mathrm{I}$; differential, 36

Adjustor: as part of reflex arc, 233; adaptive function of, 237 ; localization of, 249 . See also Arcs, reflex

"All-or-none" law. See Excitation; Transmission

Amoeba, physiological gradients in, 86

Amphibia: physiological gradients in, 33; circulation in ovarian egg of, 56 ; susceptibility in early development of, I 35

Annelids: susceptibility in, 34 ; electrical gradient in, 45 ; origin of segments in, I32; centralization in, I46; physiological dominance in, 249. See also Segmentation

Anteroposterior development, law of, 28

Arcs, excitation: definition of, 236 ; different degrees of development of, in same individual, 242; functional axiation in, 242 , 245; structural axiation in, 247 . See also Excitation; Transmission

Arcs, reflex: functional irreversibility of, 229; constitution of, $233,248,249$; adaptive character of, 233,236 ; in relation to excitation gradients, 234 ; views concerning evolution of, 237; in relation to neuro-muscle cell, 240; functional direction of, in annelids, 249; postcephalic, in higher animals, $25 \mathrm{I}$; in amphibian development, $25^{2}$; spinal paths of, in higher vertebrates, 259. See also Arcs, excitation; Gradients, excitation.

Axon, chaps. $x, x i$.

Begonia, gradients in adventitious buds of, 53

Bryopsis, determination of polarity by light in, 51

Cassiopea, transmission in, 225, 246

Centralization, nervous; definition of, 142 ; in relation to organismic pattern, I44; characteristic of all nervous systems, I44; evolutionary changes in, I 46, I 53

Cephalization, nervous, definition of, I42. See also Centralization

Cerianthus, axial integation in, 248

Conduction, 207. See also Transmission

Conductor: as part of reflex arc, 233; in primitive gradient, 234 . See also Excitation; Transmission

Correlation, chemical: significance of, I0, I2, I3; nature of, $\mathrm{I}_{3}$; in relation to organismic pattern, I4; in relation to external factors, $\mathrm{I}_{4}$; in relation to differentiation, 93 ; in relation to origin of nervous system, IO7

Correlation, contactual, I 2

Correlation, dynamic, I5. See also Transmission

Correlation, mechanical, I 2 
Correlation, physiological: organismic and protoplasmic, II ; three groups of, I 2

Correlation, transmissive, 15. See also Transmission

Correlation, transportative, ro, I 2 , 13. See also Correlation, chemical

Cortex, cerebral: pyramidal cells of, $162,166,171$; origin of, in relation to upward nerve paths, 26I; physiological characteristics of, 263 ; electrical conditions as factor in development of, 263

Crystal: organism as, 8 ; specific forms of haemoglobin, 8

Cyanide, potassium, action of, 29

Cyclopia, as differential inhibition, 38

Decrement, 67. See also Excitation; Transmission

Dedifferentiation: in consequence of physiological isolation, I03, limitation of, in higher forms, I05; in relation to tumors, I05

Dendrite, chaps. $\mathrm{x}, \mathrm{xi}$; in relation to functional irreversibility of reflex arc, 228

Dendrocoelum, head formation in pieces of, I 34

Depolarization, electric. See Polarization, electric

Differentiation: of organismic magnitude, 5; as a basis for chemical correlation, $\mathrm{I} 4$; in relation to susceptibility, 34 ; of nucleus, $6 \mathrm{I}$; in relation to physiological gradients, 90 ; in relation to rate of intake and oxidation, 92 ; in animal egg, 94; in relation to symmetry, 96 ; of nervous structure in relation to physiological gradients, Io8, II 3 , I I 5,246 ; course of, along axes, I I 9 , of neuron, chaps. $\mathrm{x}, \mathrm{xi}$; of receptor, conductor and effector, 238
Dominance, physiological: in relation to physiological gradients, 97,100 ; independent of nervous system, 99; in plants, 99; in Planaria, Ioo; persistence of, independent of gradient, I02; range and limit of, 102 , IO4; as physiological independence, I I 8; in ctenophore plate row, 212 ; in vertebrate heart, 22I; in relations of neurons, 230; in annelids, 249; change in character of, in higher vertebrates, 259; in cerebral cortex, 266. See also Gradients, physiological

Effector: ctenophore plate row not a pure, 214; as part of reflex arc, 233; in primitive gradient, 234,245 ; in neuro-muscle cell, 240; of sponges, 240; physiological impossibility of a pure, $24 \mathrm{I}$

Electrotonus, 180

Epigenetic theory, 8, 9

Equisetum, determination of polarity by light in spores of, $5 \mathrm{I}$

Excitation: in physiological correlation, 15 ; nature of, $16,67,76$, 82,207 ; as general characteristic of protoplasm, 67 ; quantitative relations in, 68, 74, 8I ; "all-or-none," 68, 75, 8I, 99, 208,223 ; theories of, 70 ; reversibility of, $75,80,82$; primitive process of, 75,80 ; specialization of mechanisms of, 79, 207, 260; in relation to physiological gradients, 82 ; as basis for qualitative changes, 93 ; in relation to nervous function, 107; development of, 207, 228; rhythmicity in, 208, 213; in neurons of different levels, 230, 258. See also Gradients, physiological; Transmission

Fucaceae, determination of polarity by light in, $5 \mathrm{I}, 60$ 
Galvanotaxis, as factor in development of neuron pattern, I 73

Gradients, axial, 24. See also gradients, physiological

Gradients, excitation, 235. See also Arc, reflex; Excitation, Gradients, physiological; Transmission

Gradients, metabolic, 24. See also Gradients, physiological

Gradients, physiological: as primary factor in axiate pattern, 24 ; as primarily superficial, 26,32 , 86; modifications of, during development, 26; evidence for existence of, 27 ; in relation to structure and development, 27; in relation to susceptibility, 32 ; reversal of, 33,243 ; in relation to rate of penetration, 40 ; in relation to reduction of $\mathrm{KMnO}_{4}$, 42 ; in relation to indophenol reaction, 44 ; in relation to electric potential, 44 ; in relation to respiration, 46 ; in axiate organs, $47,220,222$; origin of new, $5 \mathrm{I}$, 70 ; obliteration of, 52 ; in limb rudiment of Amblystoma, 52; in animal egg, 54 ; in relation to hereditary protoplasmic constitution, 64, 129, 147; not primarily concerned in evolution, 66 ; originating in excitation gradients, $70,77,82,235,239$; in Amoeba, 86; in the literature, 87 ; as an integrating factor, 89 ; in relation to differentiation, 90 , 239 ; in relation to yolk development, 94 ; in relation to dominance and subordination, 97; in relation to direction of transmission, 97, 211 , 213; in relation to physiological isolation, IO2; in relation to localization of nervous structure, 108, I44, I48, I $5 \mathrm{I}$, I53; in relation to definitely directed nerve growth, I I 2 ; nervous system primary developmental reaction to, I $_{5}$; in relation to independence and dependence, 120 ; in relation to segmentation, $132,254,260$; in annelid development, 132 ; in segmented animals in general, I35; nervous system as expression of, 144,242 ; in relation to nervous centralization, I 47,242 ; in gasteropods, 148 ; in development of axon, 168 ; in relation to direction of axon outgrowth, I85; in outgrowth of dendrites, 193; in ctenophore plate row, 220 ; in axon, 226; in sessile coelenterates, 243; in sponges, 243 ; in relation to development of amphibian reflex arcs, 252; in vertebrate development, 254 ; in higher vertebrates, 259

Harenactis: origin of new polarities in, 52; bilateral tentacle groupings in, 57 ; axial integration in, 248

Heredity: in establishment of axiate pattern, 49,58 , 59; gradient conception independent of theories of, 66,270

Heteromorphosis: axial, as normal feature of hydroid development, 34; axial, in Tubularia, 123; axial, in Planaria, 126

Hydroids: reversal of gradient in development of, 33,243 ; origin of new polarity in, 52

Inheritance, of physiological axes, 49,58

Inhibition: differential, 36 ; developmental results of, 37 ; microcephaly as differential, 37 ; cyclopia as differential, 38

Integration, organismic: nature of, 7,15 ; possible quantitative factor in mechanism of, ro; in relation to chemical correlation, 13,93 ; in relation to excitation-transmission, 89, ro7; nervous system chief organ of, 89, 90, 108; in relation to electric current, 90; following physiological isolation, 105, I35; 
in relation to nervous centralization, I42; precedes appearance of nervous system, I44; in ctenophore plate row, 2 I 2 ; in reflex arc, 233; in higher vertebrates, 259

Isolation, physiological: in relation to physiological dominance, IO2; determined in four different ways, Io3; dedifferentiation as result of, I03; in relation to agamic reproductive and repetitive processes, 104; in development of segments, I32; in development of branches of axon, I9I; in ctenophore plate row, 217

Lamarckism, not essential to gradient conception, 65,270

Material, in relation to pattern, 2. Microcephaly, as differential inhibition, 38

Neurobiotaxis, I 73, 200

Neuroblast, chaps. $\mathrm{x}$, xi

Neuro-muscle cell: polarity of, 203; as primitive receptorconductor-effector, 238, $24 \mathrm{I}$; effector of, 240

Neuron: structure and development of, chaps. $x, x i$; of spinal ganglion, I66, г96; functional and developmental polarity of, 230

Organism: pattern and material of, I; simplest forms of, 4,23 relation of, to external world, 7 ; spatial patterns of, 8; physiological correlation in, Io; as behavior pattern, 22, 268

Oxidation: in relation to physiological gradients, 25, 84; in relation to susceptibility, $3 \mathrm{I}$; in relation to excitation, $7 \mathrm{I}, 72,76$, 78,80 ; in relation to differentiation, 92

Pattern, axiate: as one of three spatial patterns in organisms, 8 ; as physiological gradient, 24 . See also Pattern, organismic

Pattern, bilateral: as one of three spatial patterns in organisms, 8; obliteration of, 56 ; origin of, 57 ; asymmetry in, 59. See also Symmetry

Pattern, cell: as surface-interior pattern, 23, 6I; nucleus as internal differentiation of, $6 \mathrm{I}$

Pattern, general: definition of, 2; distinction between, and material, 2, 270; in organism, 2; protoplasmic, 5 , I 4 ; different orders of magnitude of, 5 ; chromosomal, 6 , I8; types of, in organism, 8; excitation and transmission in, I6; relation between cytoplasmic and nuclear, I9; of neuron compared with plant, I69. See also Pattern, organismic

Pattern, neuronic: development and structure of, chaps. $x$, xi; in relation to chemotaxis, 170; as galvanotactic reaction, I II; in relation to electrical polarization, chap. xi

Pattern, organismic: definition of, 3 ; order of magnitude of, 5, I I, 85 ; origin of, $6,14,17,19,50$; preformistic theory of, 6 ; vitalistic theory of, 7; epigenetic theory of, 7,9 ; in relation to external world, 7 , I 4, 50; quantitative character of, $8,9,24,86$; three chief types of spatial, 8; as a basis for chemical correlation, I3; excitation and transmission primary factors of, 17 , 21,268 ; in relation to chromosomes, I7; surface interior, $23,61,87$, I52; axiate, 24; bilateral, 26; as physiological gradients, 27 ; precedes appearance of nervous system, I44; not necessarily adaptive, 236 . See also Gradients, physiological Pattern, protoplasmic, 3. See also Pattern, general; Pattern, organismic 
Pattern, radiate: as one of three spatial patterns in organism, 8; surface-interior pattern as, 23; repetition of parts in, 57. See also Pattern, organismic

Pattern, surface-interior, 23, 6r. See also Pattern, organismic.

Permeability: gradients in, 40 ; in relation to susceptibility, $4 \mathrm{I}$; in relation to excitation, 72 ; in relation to origin of neuron pattern, I 75

Planaria: susceptibility relations in, 34 ; external and internal gradients in, 35, 46; respiration gradients in, 47 ; biaxial heads in, $5 \mathrm{I}$; physiological dominance in, Ioo; physiological independence of head region in, I 2 I, I 25 , I 26; central nervous system of, I43, I 49

Plants: physiological gradients in, $27,29,40,47,5 \mathrm{I}, 53$; determination of polarity by light in, 5I, 6o; dominance and subordination in, 99 ; physiological isolation in, ro4; absence of nervous system in, II6; disappearance of physiological gradients in, 226

Polarity, physiological, 24, 50. See also Dominance, physiological; Gradients, physiological; Pattern, organismic

Polarization, electric: in relation to excitation, 72,180 ; in relation to neuron pattern, chap. xi; in relation to electrotonus, 180

Polyclads: reversal of susceptibility in, 34; limitation of regeneration in, I34

Potential, electric: axial gradients in, 44 ; in relation to excitation, 70,260 ; in relation to oxidationreduction, 76, I80; in relation to neuron pattern, I 73, 256, 258; in relation to development of cortex, 263. See also Polarization, electric
Preformistic theory: nature of, 6,8 ; implications of, 7,9

Protoneuron, I56. See also Reticulum, nervous

Purkinje cell, I64, I66

Pyramidal cell, I62, I66, I7 I

Receptors, position of, in relation to physiological gradients, I Io; in anaxiate form, I44; evolutionary change in position of peripheral, I5I; in reflex arc, 233 ; in primitive gradient, 234 ; localization of, in relation to axes, 247, 249

Recovery, differential, 36

Reduction, of $\mathrm{KMnO}_{4}$ in relation to physiological gradients, 42

Respiration: in relation to physiological gradients, 46; in Planaria dorotocephala, 47; in ground nervous tissue, 48

Reticulum, nervous: morphological character of, 156 ; development of pattern of, 202; functional direction in, 245

Rhythms: in excitation, 208, 213 , $2 \mathrm{I} 8$; in relation to physiological gradients, 218 ; in heart, $22 \mathrm{I}$

Segmentation: two conceptions of, I30; as a repetitive process, I3I; in relation to physiological gradients, I32, 254, 260; probable ectodermal origin of, I35, I4I, of central nervous system, I38

Self-differentiation: in embryonic development, I 20 ; of head and apical region, I2I; of central nervous system, I 27

Sponges: origin of new polarity in, 52; myocytes of, 240, 243

Stenostomum, physiological differences in asexual and sexual stages of, I 34

Sternaspis, egg polarity in, 54

Subordination, physiological, 97 . See also Dominance, physiological. 
Summation, in reflex arc, 229

Susceptibility: agents used in determining, 29; non-specific relation of, to physiological condition, 30, 36, 40; gradients in, 32 ; changes in, during development, 33; specific, 34 ; of Planaria dorotocephala, 34 ; modification of development through differential, 36 ; method of measuring, not exact, 38 ; in relation to permeability, $4 \mathrm{I}$; of embryonic heart, $48,22 \mathrm{I}$; of alimentary tract, 48 ; of ctenophore plate row, 216 ; gradient in axon, 227 ; of excitation mechanism, 228

Symmetry: similarities of, in plants and animals, 2 ; radial and bilateral, 8, 25; spherical, 23; physiological gradients in bilateral, 28 ; as susceptibility gradients, 33; bilateral, in siphonophores, 33 ; origin of, 56 ; in relation to differentiation in general, 96 ; in relation to nervous structure, I го, I49; bilateral, in relation to dorsiventral- ity, I50. See also Pattern, bilateral; Pattern, cell; Pattern, organismic; Pattern, radiate

Synapse: morphological character of, 155 ; function of, 228

Transmission: in physiological correlation, I 5; with decrement, $67,69,75,77,8$ r, 208, 21 8, 223, 229; without decrement, 68, 75, $8 \mathrm{r}, 99,208,223,225,246$; in primitive excitation processes, 77, 207, 224; up and down gradients, 97, 99, 21 1 , 218, $224,232,249$; conduction as specialized, 207 ; in ciliated epithelia, 209; in ctenophore plate row, $2 \mathrm{I}_{2}$; reversal in direction of, 2I 7, 22I; differences in rate of, 219; in relation to synapse, 229; functional irreversibility in direction of interneuronic, 229; in dendrite, $23 \mathrm{I}$

Tubularia, physiological independence of apical region in, 123

Vitalistic theory, 7, 9 


\section{The University of Chicago Science Series}

EDITORIAL COMMITTEE

ELIAKIM HASTINGS MOORE, Chairman JOHN MERLE COULTER ROBERT ANDREWS MILLIKAN

The Evolution of Sex in Plants. By John Merle Coulter. x+r40 pages, I $2 \mathrm{mo}$, cloth.

Individuality in Organisms. By Chardes Manning Child. x+2Iz pages, small I $2 \mathrm{mo}$, cloth.

The Origin of the Earth. By Thomas C. Chamberin. xii +272 pages, small r $2 \mathrm{mo}$, cloth.

Finite Collineation Groups. By Hans F. Blichfeldt. xii+ig4 pages, small r $2 \mathrm{mo}$, cloth.

A Chemical Sign of Life. By ShIro Tashiro. $\mathrm{x}+\mathrm{I}_{42}$ pages, small I $2 \mathrm{mo}$, cloth.

The Biology of Twins. By Horatio Hackett Newman. x+r86 pages, small I $2 \mathrm{mo}$, cloth.

Food Poisoning. By Edwin Oakes Jordan. viiitir6 pages, small I $2 \mathrm{mo}$, cloth.

The Electron: Its Isolation and Measurement, and the Determination of Some of Its Properties. By Robert Andrews Millikan. xii + 268 pages, small $12 \mathrm{mo}$, cloth.

The Living Cycads. By Charles Joseph Chamberlain. xiv十 72 pages, small r $2 \mathrm{mo}$, cloth.

Problems of Fertilization. By Frank R. LILLIE. xii+278 pages, small I $2 \mathrm{mo}$, cloth.

The Origin and Development of the Nervous System from a Physiological Viewpoint. By Charles M. Child. $\mathrm{x}+25^{8}$ pages, small i $2 \mathrm{mo}$, cloth.

\section{VOLUMES PLANNED OR IN PREPARATION}

Mechanics of Delayed Germination in Seeds. By W. CrockER.

Black-Body Radiation. By Charles E. Mendenhall.

The Rigidity of the Earth and of Materials. By A. A. Michelson.

Linear Integral Equations in General Analysis. By Eliakrm Hastings MOORE. 


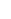




$$
\text { - }
$$




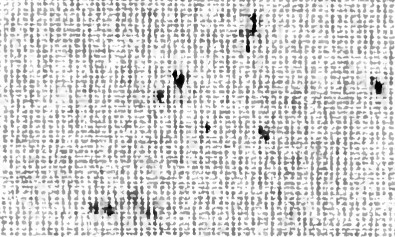

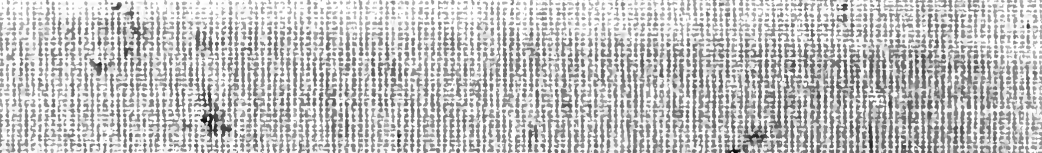

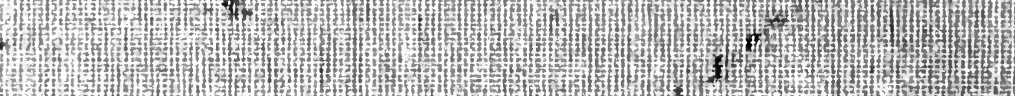

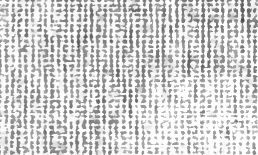
mint

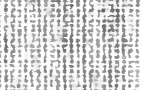

\section{${ }^{4}$}

w

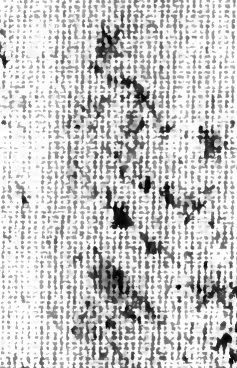

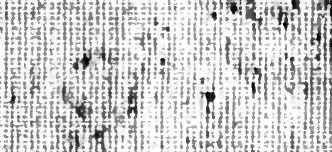

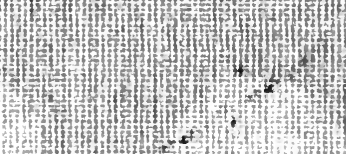

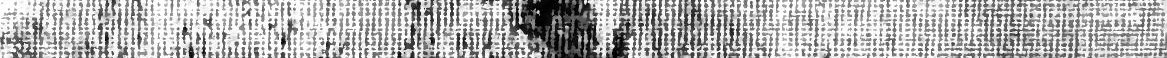

Ho 